\title{
Modular Synthesis of Alkenyl Sulfamates and $\beta$-Ketosulfonamides via Sulfur(VI) Fluoride Exchange (SuFEx) Click Chemistry and Photomediated 1,3-Rearrangement
}

\author{
Felipe Cesar Sousa e Silva, ${ }^{\dagger}$ Katarzyna Doktor, ${ }^{\dagger}$ Quentin Michaudel* \\ Department of Chemistry, Texas A\&M University, College Station, Texas 77843, United States \\ Supporting Information
}

\section{Experimental Procedures}

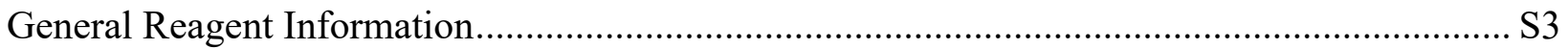

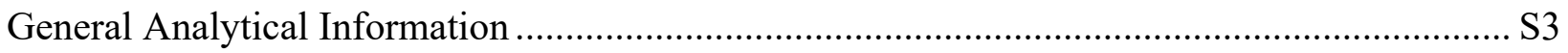

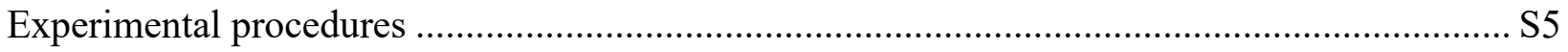

Preparation of Starting Materials ......................................................................................... S5

Optimization of the Synthesis of Alkenyl Fluorosulfonates .......................................................... S9

Synthesis of Alkenyl Fluorosulfonates: General Procedure A …………………………...... S11

Synthesis of Alkenyl Fluorosulfonates: General Procedure B ............................................... S1

Synthesis and Characterization of Isolated Alkenyl Fluorosulfonates ........................................ S12

Synthesis of Alkenyl Sulfamates: General Procedure C........................................................ S14

Synthesis and Characterization of Alkenyl Sulfamates ........................................................... S14

Representative Synthesis of Alkenyl Sulfamates (1 mmol scale) ........................................... S28

Chiral HPLC Analysis of Alkenyl Sulfamates (S)-6 and rac-6 ........................................... S29

Chiral HPLC Analysis of Alkenyl Sulfamates (S)-15 and rac-15 ........................................ S30

Attempts to Induce the Rearrangement with a Lewis or Brønsted Acid ................................... S31

Optimization of the Photochemical 1,3- Rearrangement with substrates 4 and $3 \ldots \ldots \ldots \ldots \ldots \ldots \ldots . . .531$

Probing the Effect of Oxygen in the Photochemical 1,3- Rearrangement.................................. S33

Synthesis of $\beta$-Ketosulfonamides: General Procedure D ..................................................... S34

Synthesis and Characterization of $\beta$-Ketosulfonamides ……………………….................... S35

Representative Synthesis of $\beta$-Ketosulfonamides ( $1 \mathrm{mmol} \mathrm{scale)}$ )........................................... S47

Attempts to Induce the 1,3-Rearrangement of 2 Using AIBN as Radical Initiator .................... S47 
Testing the Stability of Alkenyl Sulfamate 3 in Basic and Acidic Aqueous Media................ S48

CuAAC Click Reaction with $\beta$-Ketosulfonamides 37 ..................................................... S49

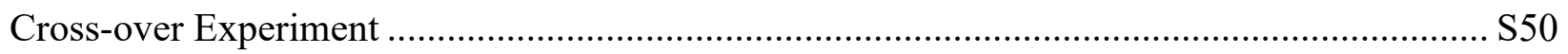

Effect of TEMPO in the Photochemical 1,3-Rearrangement …......................................... S51

Radical Clock Experiment .................................................................................................... S51

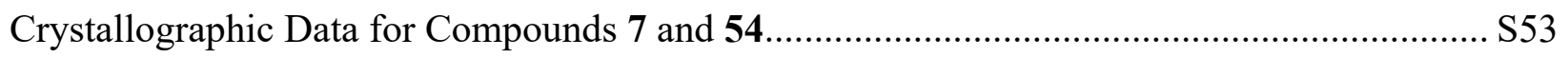

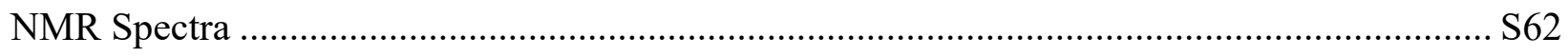




\section{General Reagent Information}

All reactions were performed without any precaution for moisture and oxygen unless otherwise stated. Dry dichloromethane (DCM), diethyl ether $\left(\mathrm{Et}_{2} \mathrm{O}\right)$, and tetrahydrofuran (THF) were obtained by passing the previously degassed solvents through activated alumina columns. Reagents were purchased at the highest commercial quality and used without further purification, unless otherwise stated. All starting materials (ketones and amines) were purchased at the highest commercial quality and used without further purification, with the exception of ketones S1, S2, and S3 and amines S4 and S5 that were synthesized following known procedures. The reactions were heated using an oil bath unless otherwise stated. Yields refer to chromatographically and spectroscopically ( ${ }^{1} \mathrm{H}$ NMR) homogeneous material, unless otherwise stated. Reactions were monitored by thin layer chromatography (TLC) carried out on $250 \mu \mathrm{m}$ SiliCycle SilicaPlate ${ }^{\mathrm{TM}}$ silica plates (F254), using UV light as the visualizing agent and an acidic solution of panisaldehyde and heat, ceric ammonium molybdate and heat, or $\mathrm{KMnO}_{4}$ and heat, or ninhydrin and heat as developing agents. Flash silica gel chromatography was performed using SiliCycle

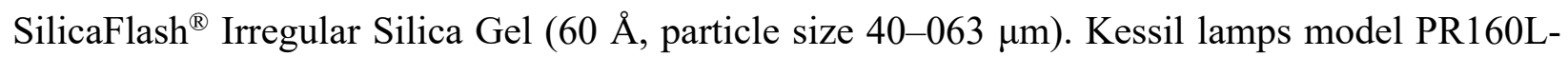
$456 \mathrm{~nm}$ with an average intensity $137 \mathrm{~mW} / \mathrm{cm}^{3}$ were used for the photochemical reactions. All photochemical reactions were performed in borosilicate glass.

\section{General Analytical Information}

${ }^{1} \mathrm{H}$ Nuclear magnetic resonance (NMR) spectra were recorded on two Bruker Avance NEO 400 $\mathrm{MHz}$ and a Bruker Avance $500 \mathrm{MHz} ;{ }^{13} \mathrm{C}$ spectra were recorded on a Bruker Avance $500 \mathrm{MHz}$;

${ }^{19} \mathrm{~F}$ spectra were recorded using a Bruker Avance NEO $400 \mathrm{MHz}$ spectra were recorded on Bruker Avance Neo 400 instrument. All ${ }^{1} \mathrm{H}$ and ${ }^{13} \mathrm{C}$ spectra were calibrated using residual deuterated solvent as an internal reference $\left(\mathrm{CDCl}_{3} @ 7.26\right.$ ppm ${ }^{1} \mathrm{H}$ NMR, 77.16 ppm ${ }^{13} \mathrm{C} \mathrm{NMR}$; $\mathrm{d}_{6}-\mathrm{DMSO}$ @ 2.50 ppm ${ }^{1} \mathrm{H}$ NMR, $\left.39.52 \mathrm{ppm}{ }^{13} \mathrm{C} \mathrm{NMR} ; \mathrm{C}_{2} \mathrm{D}_{2} \mathrm{Cl}_{4} @ 6.00 \mathrm{ppm}{ }^{1} \mathrm{H} \mathrm{NMR}, 73.78 \mathrm{ppm}{ }^{13} \mathrm{C} \mathrm{NMR}\right)$. The following abbreviations were used to explain NMR peak multiplicities: $s=$ singlet, $d=$ doublet, $\mathrm{t}=$ triplet, $\mathrm{q}=$ quartet, $\mathrm{m}=$ multiplet, $\mathrm{br}=$ broad. High-resolution mass spectra (HRMS) were recorded on an Agilent LC/MSD TOF mass spectrometer by electrospray ionization time-offlight (ESI-TOF) reflection experiments. Melting points were recorded on a Fisher-Johns 13-144 melting point apparatus and are uncorrected. Crystal structures were collected on a Bruker Photon 2 kappa microsource diffractometer. The crystal structures of 7 and $\mathbf{5 4}$ have been deposited at the 
Cambridge Crystallographic Data Centre: CCDC 2083389 (for 7), CCDC 2083388 (for 54). Chiral HPLC was performed with an Agilent 1260 Infinity II HPLC and a Chiralcel(R) OD-H column that is $4.6 \mathrm{~mm}$ I.D. $\mathrm{x} 250 \mathrm{~mm}$ in length and particle size of $5 \mu \mathrm{m}$. 


\section{Experimental procedure}

\section{Preparation of Starting Materials}

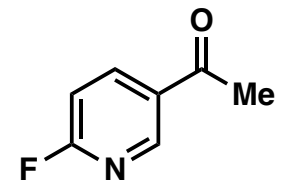

S1

1-(6-Fluoropyridin-3-yl)ethanone (S1). S1 was synthesized following a previously reported procedure ( $J$. Med. Chem. 2020, 63, 5879) with slight modifications. An oven dry $50 \mathrm{~mL}$ round-bottom-flask was charged with 2 fluoro-5-pyridinecarboxylic acid (0.50 g, $3.54 \mathrm{mmol}, 1.00$ equiv), 1hydroxybenzotriazole $\quad(0.81 \quad \mathrm{~g}, \quad 5.40 \quad \mathrm{mmol}, \quad 1.49 \quad$ equiv), 1-ethyl-3-(3dimethylaminopropyl)carbodiimide hydrochloride (1.0 g, $5.31 \mathrm{mmol}, 1.50$ equiv) and dry DCM $(7.0 \mathrm{~mL})$ was added to the flask. The slurry turned into a homogenous solution after addition of $N, N$-diisopropylethylamine (1.85 mL, $1.37 \mathrm{~g}, 10.62 \mathrm{mmol}, 3.00$ equiv), which was stirred for 10 $\min$ at $0{ }^{\circ} \mathrm{C}$. Then, $N, O$-dimethylhydroxylamine hydrochloride $(0.52 \mathrm{~g}, 5.31 \mathrm{mmol}, 1.49$ equiv $)$ was added as a solid in one portion. After stirring for $16 \mathrm{~h}$ at room temperature, the mixture was diluted with DCM $(10 \mathrm{~mL})$ and washed with cold water $(10 \mathrm{~mL})$ and brine $(10 \mathrm{~mL})$. The organic layers were dried over $\mathrm{MgSO}_{4}$ and concentrated in vacuo. Dry THF (7.0 mL) was added to the dry crude material $\left(0.50 \mathrm{~g}, 2.70 \mathrm{mmol}, 1.00\right.$ equiv) under nitrogen and the solution was cooled to $0{ }^{\circ} \mathrm{C}$. A solution of methyl magnesium bromide in $\mathrm{Et}_{2} \mathrm{O}(2.70 \mathrm{~mL}, \mathrm{C}=3.0 \mathrm{M}, 8.10 \mathrm{mmol}, 1.00$ equiv) was added slowly over 20 minutes and the mixture was stirred for another $3 \mathrm{~h}$ while monitoring by TLC. The mixture was then cooled to $0{ }^{\circ} \mathrm{C}$ and $a q . \mathrm{NH}_{4} \mathrm{Cl}(5 \mathrm{~mL})$ was added slowly until gas formation ceased. The quenched reaction was allowed to warm to room temperature, extracted with EtOAc $(3 \times 8 \mathrm{~mL})$, and washed with brine $(8 \mathrm{~mL})$. Crude material was purified by column chromatography $\left(\mathrm{SiO}_{2}, 20: 80\right.$ EtOAc:hexanes) to afford a white solid $(0.32 \mathrm{~g}, 64 \%$ over two steps).

The spectroscopic data for this compound were identical to those reported in the literature (Adv. Synth. Catal. 2017, 359, 2, 279).

${ }^{1} \mathrm{H} \mathrm{NMR}\left(\mathrm{CDCl}_{3}, 400 \mathrm{MHz}\right) \delta: 8.80(\mathrm{~d}, J=2.4 \mathrm{~Hz}, 1 \mathrm{H}), 8.36(\mathrm{ddd}, J=8.5 \mathrm{~Hz}, J=7.7 \mathrm{~Hz}, J=$ $2.6 \mathrm{~Hz}, 1 \mathrm{H}), 7.03(\mathrm{dd}, J=8.6,2.8 \mathrm{~Hz}, 1 \mathrm{H}), 2.63$ (s, $3 \mathrm{H}) \mathrm{ppm}$. 


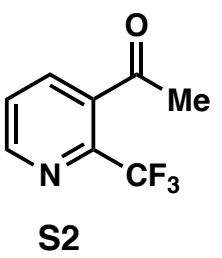

1-[2-(trifluoromethyl)pyridin-3-yl]ethan-1-one

following the same procedure as for $\mathbf{S 1}$ starting with 2-(trifluoromethyl)nicotinic acid ( $0.68 \mathrm{~g}, 3.54 \mathrm{mmol}, 1.00$ equiv). The crude material was purified by column chromatography $\left(\mathrm{SiO}_{2}, 10: 90\right.$ EtOAc:hexanes) to afford a white solid $(0.59 \mathrm{~g}, 88$

The spectroscopic data for this compound were identical to those reported in the literature (Patent WO2016/132134, 2016, A1).

${ }^{1} \mathrm{H} \operatorname{NMR}\left(\mathrm{CDCl}_{3}, 400 \mathrm{MHz}\right) \delta: 8.67(\mathrm{~d}, J=4.3 \mathrm{~Hz}, 1 \mathrm{H}), 7.75(\mathrm{~d}, J=7.7 \mathrm{~Hz}, 1 \mathrm{H}), 7.51$ (dd, $J=$ 7.7, $4.7 \mathrm{~Hz}, 1 \mathrm{H}), 2.50$ (s, $3 \mathrm{H}) \mathrm{ppm}$.

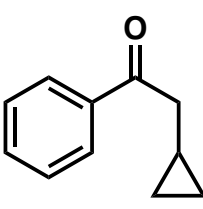

S3

2-Cyclopropyl-1-phenylethanone (S3). S3 was prepared following a previously reported procedure (U.S. Patent 0147559, July 29, 2004, pp 176) with slight modifications. A flame-dried $50 \mathrm{~mL}$ round-bottom-flask was charged with cyclopropylacetonitrile $\left(0.50 \mathrm{~g}, 6.16 \mathrm{mmol}, 1.00\right.$ equiv) and dry $^{\mathrm{Et}} \mathrm{t}_{2} \mathrm{O}(25 \mathrm{~mL})$ was added to the flask. After the solution was cooled to $0^{\circ} \mathrm{C}$, a solution of phenyl magnesium bromide in $\mathrm{Et}_{2} \mathrm{O}(0.92 \mathrm{~mL}, \mathrm{C}=3.0 \mathrm{M}, 2.75 \mathrm{mmol}, 1.10$ equiv) was added slowly over 20 min under nitrogen and the mixture was allowed to stir for $3 \mathrm{~h}$ at $0{ }^{\circ} \mathrm{C}$. Then, $a q . \mathrm{HCl}(1 \mathrm{M}, 5 \mathrm{~mL})$ was added and the reaction was stirred at room temperature for $16 \mathrm{~h}$. After the reaction was completed, the mixture was diluted with DCM $(40 \mathrm{~mL})$ and washed with water. Organic layers were washed with brine (15 mL), dried over $\mathrm{MgSO}_{4}$, and concentrated in vacuo. Column chromatography $\left(\mathrm{SiO}_{2}\right.$, hexanes) afforded a colorless oil $(0.99 \mathrm{~g}, 32 \%)$.

The spectroscopic data for this compound were identical to those reported in the literature (Eur. J. Org. Chem. 2006, 11, 2601).

${ }^{1} \mathrm{H} \mathrm{NMR}\left(\mathrm{CDCl}_{3}, 400 \mathrm{MHz}\right) \delta:$ 7.98-7.91 (m, $\left.2 \mathrm{H}\right), 7.59-7.52$ (m, $\left.1 \mathrm{H}\right), 7.52-7.42$ (m, $\left.2 \mathrm{H}\right), 2.89$ (d, $J=6.8 \mathrm{~Hz}, 2 \mathrm{H}), 1.11-1.24(\mathrm{~m}, 1 \mathrm{H}), 0.57-0.64$ (m, $2 \mathrm{H}), 0.17-0.22$ (m, $2 \mathrm{H}) \mathrm{ppm}$.

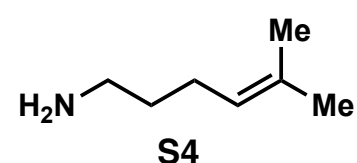

S4

5-methylhex-4-en-1-amine (S4). S4 was prepared following a previously reported procedure (J. Am. Chem. Soc. 2020, 142, 13, 5974). To an ovendried $250 \mathrm{~mL}$ round acetonitrile $(0.75 \mathrm{~mL}, 14.1 \mathrm{mmol}, 1.05$ equiv) and THF 
$(55 \mathrm{~mL})$ were added under nitrogen. The mixture was cooled to $-78{ }^{\circ} \mathrm{C}$ and a solution of ${ }^{n} \mathrm{BuLi}$ in hexanes $(5.80 \mathrm{~mL}, \mathrm{C}=2.5 \mathrm{M}, 14.5 \mathrm{mmol}, 1.10$ equiv) was added dropwise over $20 \mathrm{~min}$. After stirring for $30 \mathrm{~min}$, 1-bromo-3-methylbut-2-ene (2.00 g, $13.4 \mathrm{mmol}, 1.00$ equiv) was added dropwise. The mixture was allowed to warm to room temperature overnight, after which the THF was removed carefully in vacuo. The residues were transferred to a separatory funnel and extracted with $\mathrm{Et}_{2} \mathrm{O}(3 \times 15 \mathrm{~mL})$. The combined organic layers were washed with brine $(20 \mathrm{~mL})$, dried over $\mathrm{MgSO}_{4}$, and concentrated in vacuo. The crude material was used directly in the next step without further purification.

A round bottom flask containing 5-methylhex-4-enenitrile was dried under vacuo for $1 \mathrm{~h}$ and dry $\mathrm{Et}_{2} \mathrm{O}(40 \mathrm{~mL})$ was added to the flask. Next, $\mathrm{LiAlH}_{4}(1.50 \mathrm{~g}, 20.1 \mathrm{mmol}, 1.50$ equiv) was added slowly over $20 \mathrm{~min}$ at $0^{\circ} \mathrm{C}$ in a solution of dry $\mathrm{Et}_{2} \mathrm{O}(15 \mathrm{~mL})$ and the reaction was allowed to be warmed to room temperature gradually overnight $(16 \mathrm{~h})$. The mixture was quenched following the Fieser procedure with sequential dropwise addition of water $(1.5 \mathrm{~mL})$, aq. sodium hydroxide $(10 \%, 1.5 \mathrm{~mL})$, and water $(4.5 \mathrm{~mL})$, which led to the formation of a white suspension. Then, magnesium sulfate was added slowly to the flask at $0{ }^{\circ} \mathrm{C}$ over $15 \mathrm{~min}$. After stirring for additional $15 \mathrm{~min}$ at room temperature and the mixture was filtered over a plug of Celite and solvent was removed under the reduced pressure. The isolated material was used without further purification.

The spectroscopic data for this compound were identical to those reported in the literature (Chem. Ber. 1989, 122, 997).

${ }^{1} \mathrm{H}$ NMR $\left(\mathrm{CDCl}_{3}, 400 \mathrm{MHz}\right) \delta: 5.12(\mathrm{~m}, 1 \mathrm{H}), 2.69$ (t, $\left.J=7.0 \mathrm{~Hz}, 2 \mathrm{H}\right), 2.03(\mathrm{q}, J=7.0 \mathrm{~Hz}, 2 \mathrm{H})$, $1.70(\mathrm{~s}, 3 \mathrm{H}), 1.62(\mathrm{~s}, 3 \mathrm{H}), \delta 1.46(\mathrm{~h}, J=7.1 \mathrm{~Hz}, 2 \mathrm{H}), 1.10(\mathrm{td}, J=7.0,1.6 \mathrm{~Hz}, 2 \mathrm{H}) \mathrm{ppm}$.

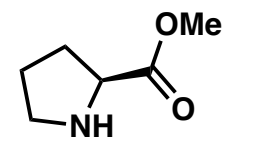

S5

L-proline methyl ester (S5). S5 was prepared following a previously reported procedure with a slight modification (J. Am. Chem. Soc. 2020, 142, 3, 1526.) A flame dried $25 \mathrm{~mL}$ round-bottom-flask was charged with L-proline (2.48 g, 21.5 mmol, 1.00 equiv) and anhydrous $\mathrm{MeOH}(20 \mathrm{~mL}, \mathrm{C}=1.0 \mathrm{M})$ was added to the flask. After the solution was cooled to $0^{\circ} \mathrm{C}$, thionyl chloride $(2.50 \mathrm{~mL}, 34.5 \mathrm{mmol}, 1.60$ equiv) was added slowly over $10 \mathrm{~min}$ under nitrogen and the mixture was allowed to stir for $16 \mathrm{~h}$ at room temperature. Then, the solvent was removed under reduced pressure and the remaining oil was diluted with EtOAc 
$(10 \mathrm{~mL})$ to be transferred to separatory funnel. Next, aq. $\mathrm{K}_{2} \mathrm{CO}_{3}(20 \mathrm{~mL})$ was added to naturalize the solution, which was extracted with EtOAc $(3 \times 10 \mathrm{~mL})$. The organic layers were washed with water $(15 \mathrm{~mL})$, dried over $\mathrm{MgSO}_{4}$, and solvent was removed under the reduced pressure to afford brown oil. Crude material was used without further purification.

The spectroscopic data for this compound were identical to those reported in the literature $(\mathrm{J} . \mathrm{Am}$. Chem. Soc. 2020, 142, 3, 1526).

${ }^{1} \mathrm{H}$ NMR $\left(\mathrm{CDCl}_{3}, 400 \mathrm{MHz}\right) \delta: 3.66(\mathrm{dd}, J=8.6,5.6 \mathrm{~Hz}, 1 \mathrm{H}), 3.65(\mathrm{~s}, 3 \mathrm{H}), 2.95(\mathrm{dt}, J=9.9$, $6.6 \mathrm{~Hz}, 1 \mathrm{H}), 2.80(\mathrm{dt}, J=10.1,6.6 \mathrm{~Hz}, 1 \mathrm{H}), 2.27(\mathrm{~s}, 1 \mathrm{H}), 2.07-1.97$ (m, $1 \mathrm{H}), 1.81-1.65(\mathrm{~m}$, $3 \mathrm{H}) \mathrm{ppm}$.

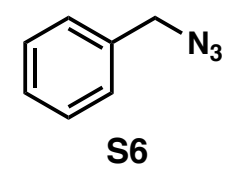

Benzyl azide (S6). S6 was prepared following a previously reported procedure with a slight modification (Org. Lett. 2020, 22, 6, 2376). A flame-dried $25 \mathrm{~mL}$ roundbottom-flask was charged with benzyl bromide (1.19 mL, $15.0 \mathrm{mmol}, 1.00$ equiv) and dry DMF (75 mL, C =0.2 M) was added to the flask. Sodium azide (0.98 g, $15.0 \mathrm{mmol}, 1.00$ equiv) was added and the reaction was stirred for $14 \mathrm{~h}$ at $80^{\circ} \mathrm{C}$. The reaction mixture was cooled to room temperature and diluted with EtOAc $(30 \mathrm{~mL})$. The biphasic system was separated and the organic layer were washed with ice-cooled $\mathrm{H}_{2} \mathrm{O}(4 \times 30 \mathrm{~mL})$ and brine $(30 \mathrm{~mL})$. After drying over anhydrous $\mathrm{Na}_{2} \mathrm{SO}_{4}$, the solvent was removed under reduced pressure to obtain the pure product which was used without further purification.

The spectroscopic data for this compound were identical to those reported in the literature (Tetrahedron 2009, 65, 5361).

${ }^{1} \mathrm{H}$ NMR $\left(\mathrm{CDCl}_{3}, 400 \mathrm{MHz}\right) \delta: 3.66(\mathrm{dd}, J=8.6,5.6 \mathrm{~Hz}, 1 \mathrm{H}), 3.65(\mathrm{~s}, 3 \mathrm{H}), 2.95(\mathrm{dt}, J=9.9$, $6.6 \mathrm{~Hz}, 1 \mathrm{H}), 2.80(\mathrm{dt}, J=10.1,6.6 \mathrm{~Hz}, 1 \mathrm{H}), 2.27(\mathrm{~s}, 1 \mathrm{H}), 2.07-1.97$ (m, $1 \mathrm{H}), 1.81-1.65(\mathrm{~m}$, 3 H) ppm.

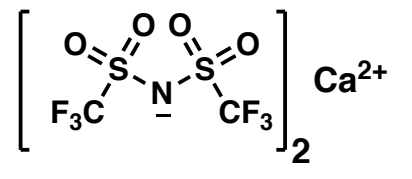

S7
Calcium bis(trifluoromethanesulfonimide) (S7). Reagent S7 was prepared following a previously reported procedure (Catal. Today 1997, 36, 81). A $100 \mathrm{~mL}$ round-bottom-flask was charged with 
trifluoromethanesulfonimide (5.62 g, $20.0 \mathrm{mmol}, 2.00$ equiv) and water was used to facilitate the transfer of the solid reagent. Then, $\mathrm{CaCO}_{3}(1.00 \mathrm{~g}, 9.99 \mathrm{mmol}, 1.00$ equiv) was added in one portion and water $(5 \mathrm{~mL})$ was used to transfer $\mathrm{CaCO}_{3}$ remaining on the weighing paper. Ensuring the exact $2: 1$ ratio of reagents was found instrumental in this procedure. The solution was stirred at room temperature for $1 \mathrm{~h}$, then water was removed under reduced pressure. The resulting solid was dried under high vacuum at $105^{\circ} \mathrm{C}$ over $12 \mathrm{~h}$ to afford a white powder $(5.62 \mathrm{~g}, 93 \%)$.

The spectroscopic data for this compound were identical to those reported in the literature (Tetrahedron 2009, 65, 5361).

${ }^{13} \mathrm{C}$ NMR $\left(d_{6}\right.$-acetone, $\left.126 \mathrm{MHz}\right) \delta: 120.0(\mathrm{q}, J=320.0 \mathrm{~Hz}) \mathrm{ppm}$.

Optimization of the Synthesis of Alkenyl Fluorosulfonates

Conditions for the synthesis of alkenyl fluorosulfonates were optimized with $\alpha$-tetralone (1) as a model substrate.

Table S1. Representative Conditions Screened for the Synthesis of Alkenyl Fluorosulfonate 2

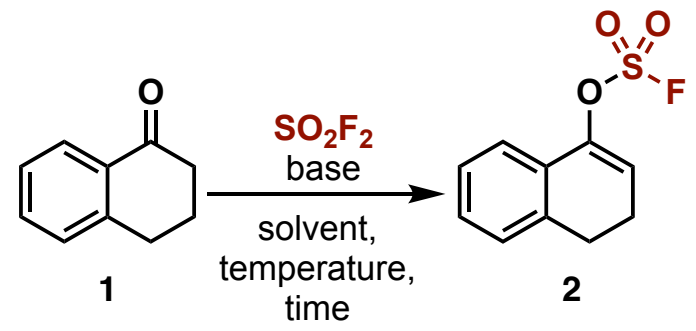

\begin{tabular}{cccccc} 
Entry & Base & Solvent & Temperature & Time & Yield $^{a}$ \\
\hline 1 & LDA (1.2 equiv) & THF $(\mathrm{C}=0.5 \mathrm{M})$ & -78 to $0{ }^{\circ} \mathrm{C}$ & $1.5 \mathrm{~h}$ & $84 \%$ \\
2 & ${ }^{t} \mathrm{BuOK}(2.2$ equiv) & $\mathrm{THF}(\mathrm{C}=0.5 \mathrm{M})$ & -78 to $0{ }^{\circ} \mathrm{C}$ & $1.5 \mathrm{~h}$ & traces \\
3 & ${ }^{t} \mathrm{BuOK}(2.2$ equiv) & $\mathrm{Et}_{2} \mathrm{O}(\mathrm{C}=0.5 \mathrm{M})$ & $0{ }^{\circ} \mathrm{C}$ & $1.5 \mathrm{~h}$ & $51 \%$ \\
4 & ${ }^{t} \mathrm{BuOK}(2.2$ equiv) & $\mathrm{Et}_{2} \mathrm{O}(\mathrm{C}=0.5 \mathrm{M})$ & $-78^{\circ} \mathrm{C}$ & $1.5 \mathrm{~h}$ & $78 \%$ \\
$\mathbf{5}$ & ${ }^{t} \mathbf{B u O K}(\mathbf{2 . 2}$ equiv) & $\mathbf{T H F}(\mathbf{C}=\mathbf{0 . 5 M})$ & $\mathbf{- 7 8}{ }^{\circ} \mathbf{C}$ & $\mathbf{1 . 5} \mathbf{~ h}$ & $\mathbf{9 0 \%}$
\end{tabular}

The reactions were carried out using $0.15 \mathrm{mmol}$ of the starting material under nitrogen atmosphere. ${ }^{a}$ Yields were determined by ${ }^{1} \mathrm{H}$ NMR using 1,4-dimethoxybenzene as internal standard.

Conditions for the synthesis of alkenyl fluorosulfonates were optimized with 3-acetylpyridine as a model substrate. 
Table S2. Representative Conditions Screened for the Synthesis of Alkenyl Fluorosulfonate S9

\begin{tabular}{|c|c|c|c|c|c|c|}
\hline Entry & Base (equiv) & Solvent & Conc. & Temperature & Time & Yield \\
\hline 1 & ${ }^{t} \mathrm{BuOK}(2.2)$ & THF & $0.2 \mathrm{M}$ & $0{ }^{\circ} \mathrm{C}$ & $1.5 \mathrm{~h}$ & $42 \%$ \\
\hline 2 & ${ }^{t} \mathrm{BuONa}(2.2)$ & THF & $0.2 \mathrm{M}$ & $0{ }^{\circ} \mathrm{C}$ & $1.5 \mathrm{~h}$ & $46 \%$ \\
\hline 3 & $\mathrm{NaH}(2.2)$ & THF & $0.2 \mathrm{M}$ & $0{ }^{\circ} \mathrm{C}$ & $1.5 \mathrm{~h}$ & $<5 \%$ \\
\hline 4 & ${ }^{n} \operatorname{BuLi}(2.2)$ & THF & $0.2 \mathrm{M}$ & $0{ }^{\circ} \mathrm{C}$ & $1.5 \mathrm{~h}$ & - \\
\hline 5 & LDA (1.2) & THF & $0.5 \mathrm{M}$ & $-78^{\circ} \mathrm{C}$ & $1 \mathrm{~h}$ & $22 \%$ \\
\hline 6 & ${ }^{t} \mathrm{BuOK}$ (1.2) & THF & $0.2 \mathrm{M}$ & $0{ }^{\circ} \mathrm{C}$ & $1.5 \mathrm{~h}$ & $17 \%$ \\
\hline 7 & ${ }^{t} \mathrm{BuOK}$ (3.2) & THF & $0.2 \mathrm{M}$ & $0{ }^{\circ} \mathrm{C}$ & $1.5 \mathrm{~h}$ & $42 \%$ \\
\hline 8 & ${ }^{t} \mathrm{BuOK}(2.2)$ & $\mathrm{MeCN}$ & $0.2 \mathrm{M}$ & $0{ }^{\circ} \mathrm{C}$ & $1.5 \mathrm{~h}$ & - \\
\hline 9 & ${ }^{t} \mathrm{BuOK}(2.2)$ & DMF & $0.2 \mathrm{M}$ & $0{ }^{\circ} \mathrm{C}$ & $1.5 \mathrm{~h}$ & - \\
\hline 10 & ${ }^{t} \mathrm{BuOK}(2.2)$ & $\mathrm{Et}_{2} \mathrm{O}$ & $0.2 \mathrm{M}$ & $0{ }^{\circ} \mathrm{C}$ & $1.5 \mathrm{~h}$ & $63 \%$ \\
\hline 11 & ${ }^{t} \mathrm{BuOK}(2.2)$ & DCM & $0.2 \mathrm{M}$ & $0{ }^{\circ} \mathrm{C}$ & $1.5 \mathrm{~h}$ & $72 \%$ \\
\hline 12 & ${ }^{t} \mathrm{BuOK}(2.2)$ & DCM & $0.2 \mathrm{M}$ & $0{ }^{\circ} \mathrm{C}$ & $1.5 \mathrm{~h}$ & $71 \%$ \\
\hline 13 & ${ }^{t} \mathrm{BuOK}(2.2)$ & DCM & $0.4 \mathrm{M}$ & $0{ }^{\circ} \mathrm{C}$ & $1.5 \mathrm{~h}$ & $67 \%$ \\
\hline 14 & ${ }^{t} \mathrm{BuOK}$ (2.2) & DCM & $0.1 \mathrm{M}$ & ${ }^{\circ}{ }^{\circ} \mathrm{C}$ & $1 \mathrm{~h}$ & $78 \%$ \\
\hline 15 & ${ }^{t} \mathrm{BuOK}(2.2)$ & DCM & $0.1 \mathrm{M}$ & $-78^{\circ} \mathrm{C}$ & $1 \mathrm{~h}$ & $74 \%$ \\
\hline 16 & ${ }^{t} \mathrm{BuOK}(2.2)$ & DCM & $0.1 \mathrm{M}$ & $0{ }^{\circ} \mathrm{C}$ & $2 \mathrm{~h}$ & $78 \%$ \\
\hline 17 & ${ }^{t} \mathrm{BuOK}(2.2)$ & DCM & $0.1 \mathrm{M}$ & $0{ }^{\circ} \mathrm{C}$ & $30 \mathrm{~min}$ & $44 \%$ \\
\hline
\end{tabular}

The reactions were carried out using $0.15 \mathrm{mmol}$ of starting ketone under nitrogen atmosphere. 


\section{$\underline{\text { Synthesis of Alkenyl Fluorosulfonates: General Procedure A }}$}

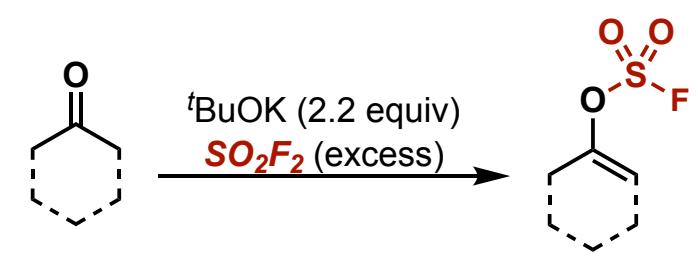

To a flame-dried $25 \mathrm{~mL}$ round-bottom-flask equipped with a stir bar and molecular sieves (3 $\AA$ ) and placed under nitrogen. ${ }^{t} \mathrm{BuOK}(37.0 \mathrm{mg}, 0.33 \mathrm{mmol}, 2.20$ equiv) and the ketone substrate $(0.15$ mmol, 1.00 equiv) were added to the flask. Anhydrous solvent $(1.5 \mathrm{~mL}, \mathrm{C}=0.1 \mathrm{M}, T H F, D C M$, or $E t_{2} \mathrm{O}$, see below for each specific substrate) was added under nitrogen and the mixture was stirred for $10 \mathrm{~min}$. The reaction was then cooled to -78 or $0{ }^{\circ} \mathrm{C}$ and the headspace was evacuated until the solvent began boiling. A balloon containing sulfuryl fluoride gas (approx. $25 \mathrm{~mL}$ in size) was introduced. After $1.5 \mathrm{~h}$ of stirring at -78 or $0{ }^{\circ} \mathrm{C}$, the molecular sieves were removed by filtration and the filtrate was diluted with distilled water $(5 \mathrm{~mL})$. The mixture was then extracted with DCM (3 x $5 \mathrm{~mL})$ and combined organic layers were washed with brine $(5 \mathrm{~mL})$ and dried over $\mathrm{MgSO}_{4}$. The solvent was evaporated in vacuo and conversions were determined by ${ }^{1} \mathrm{H} \mathrm{NMR}$. Note: Due to decomposition issues during column chromatography, some alkenyl fluorosulfonates were carried to the next step without purification, unless otherwise stated.

Synthesis of Alkenyl Fluorosulfonates: General Procedure B

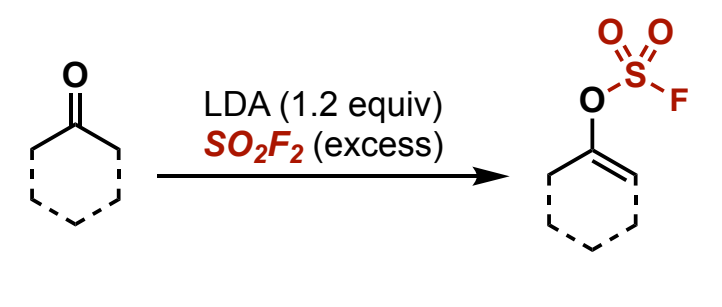

To a flame-dried $25 \mathrm{~mL}$ round-bottom-flask equipped with a stir bar and placed under nitrogen, dry diisopropylamine $(0.26 \mathrm{~mL}, 0.18 \mathrm{mmol}, 1.20$ equiv) was added followed by anhydrous THF $\left(0.30 \mathrm{~mL},(\mathrm{C}=0.5 \mathrm{M})\right.$. The mixture was cooled to $0{ }^{\circ} \mathrm{C}$, followed by slow addition of a solution of ${ }^{n} \mathrm{BuLi}$ in hexanes $(70 \mu \mathrm{L}, \mathrm{C}=2.5 \mathrm{M}, 0.17 \mathrm{mmol}, 1.10$ equiv) under nitrogen. After stirring for 
$30 \mathrm{~min}$, the mixture was cooled to $-78{ }^{\circ} \mathrm{C}$ and the ketone $(0.15 \mathrm{mmol}, 1.00$ equiv) was added dropwise over $5 \mathrm{~min}$. After addition of the ketone starting material, the headspace was evacuated until the THF began boiling, and a balloon containing sulfuryl fluoride gas (approx. $25 \mathrm{~mL}$ in size) was introduced. The reaction was then warmed up to $0{ }^{\circ} \mathrm{C}$ and was stirred at that temperature for $1 \mathrm{~h}$. Then, the reaction was quenched with sat. $\mathrm{NH}_{4} \mathrm{Cl}(15 \mathrm{~mL})$. The mixture was extracted with EtOAc $(3 \times 5 \mathrm{~mL})$ and the combined organic layers were washed with brine $(5 \mathrm{~mL})$ and dried over $\mathrm{MgSO}_{4}$. The solvent was evaporated in vacuo and the crude material was purified by column chromatography and analyzed by ${ }^{1} \mathrm{H}$ NMR. Note: Due to decomposition issues during column chromatography, some alkenyl fluorosulfonates were carried to the next step without purification, unless otherwise stated.

Synthesis and Characterization of Isolated Alkenyl Fluorosulfonates

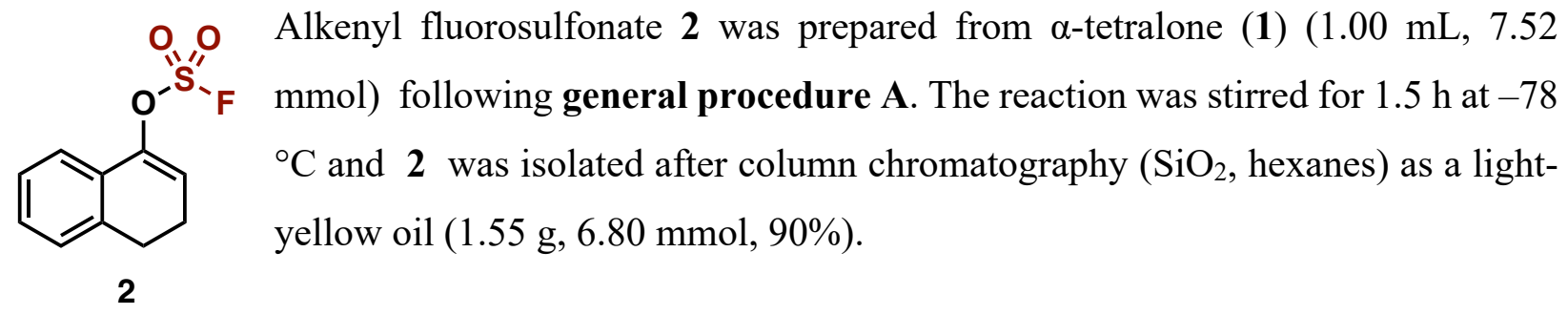

The spectroscopic data for this compound were identical to those reported in the literature (US10117840B2, 2018).

${ }^{1} \mathrm{H}$ NMR $\left(\mathrm{CDCl}_{3}, 400 \mathrm{MHz}\right) \delta 7.39-7.16(\mathrm{~m}, 4 \mathrm{H}), 6.10(\mathrm{td}, J=4.9,1.8 \mathrm{~Hz}, 2 \mathrm{H}), 2.90(\mathrm{t}, J=8.3$ $\mathrm{Hz}, 2 \mathrm{H}), 2.54$ (td, $J=8.2,4.8 \mathrm{~Hz}, 2 \mathrm{H}) \mathrm{ppm}$.

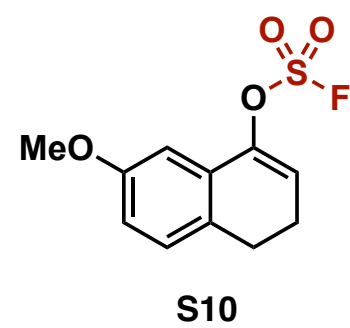
$\mathrm{mg}, 0.42 \mathrm{mmol}$ ) following general procedure $\mathbf{B}$ in $82 \%$ yield.

Alkenyl fluorosulfonate S10 was prepared from 7-methoxy-1-tetralone (100 mg, $0.57 \mathrm{mmol}$ ) following general procedure A. Anhydrous THF was used and the reaction was stirred for $1.5 \mathrm{~h}$ at $-78^{\circ} \mathrm{C}$. S10 was isolated after column chromatography $\left(\mathrm{SiO}_{2}\right.$, hexanes) as a light brown oil $(128 \mathrm{mg}$, 87\%). Alternatively, $\mathbf{S 1 0}$ can be prepared from 7-methoxy-1-tetralone (89

$\mathrm{R}_{\mathrm{f}}=0.71$ (1:4 EtOAc:hexanes) 
${ }^{1} \mathrm{H}$ NMR $\left(\mathrm{CDCl}_{3}, 400 \mathrm{MHz}\right) \delta: 7.11-7.09(\mathrm{~d}, J=8.3 \mathrm{~Hz}, 1 \mathrm{H}), 6.91-6.90(\mathrm{~d}, J=2.6 \mathrm{~Hz}, 1 \mathrm{H})$, 6.82-6.79 (dd, $J=8.3,2.6 \mathrm{~Hz}, 1 \mathrm{H}), 6.13-3.10$ (dt, $J=5.7,2.9 \mathrm{~Hz}, 1 \mathrm{H}), 3.81(\mathrm{~s}, 3 \mathrm{H}), 2.83-2.79$ $(\mathrm{t}, J=8.2 \mathrm{~Hz}, 2 \mathrm{H}), 2.53-2.48(\mathrm{td}, J=8.2,4.8 \mathrm{~Hz}, 2 \mathrm{H}) \mathrm{ppm}$.

${ }^{13} \mathrm{C} \mathrm{NMR}\left(\mathrm{CDCl}_{3}, 126 \mathrm{MHz}\right) \delta: 159.0,147.4,129.2,129.1,128.6,118.4,114.7,107.7,55.8,26.3$, 22.9 ppm.

${ }^{19} \mathrm{~F} \mathrm{NMR}\left(\mathrm{CDCl}_{3}, 470 \mathrm{MHz}\right) \delta: 39.0 \mathrm{ppm}$

HRMS(+APCI): calc'd for $\mathrm{C}_{11} \mathrm{H}_{11} \mathrm{FO}_{4} \mathrm{~S}[\mathrm{M}+\mathrm{H}]^{+} 259.0435$, found 259.0430.

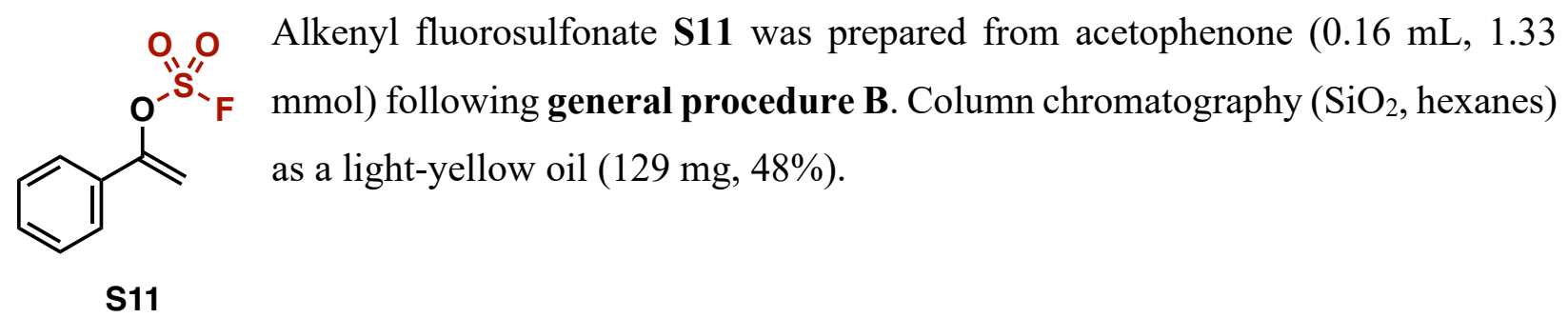

$\mathrm{R}_{\mathrm{f}}=0.74$ (1:4 EtOAc:hexanes)

${ }^{1} \mathrm{H}$ NMR $\left(\mathrm{CDCl}_{3}, 400 \mathrm{MHz}\right) \delta: 7.59-7.53(\mathrm{~m}, 2 \mathrm{H}), 7.47-7.40(\mathrm{~m}, 3 \mathrm{H}), 5.67-5.66(\mathrm{~d}, J=4.1 \mathrm{~Hz}$, $1 \mathrm{H}), 5.47-5.46$ (dd, $J=4.1,1.4 \mathrm{~Hz}, 1 \mathrm{H}) \mathrm{ppm}$.

${ }^{13} \mathrm{C} \mathrm{NMR}\left(\mathrm{CDCl}_{3}, 126 \mathrm{MHz}\right) \delta: 154.3,131.2,130.6,129.0,125.5,103.9,103.9 \mathrm{ppm}$.

${ }^{19} \mathrm{~F} \mathrm{NMR}\left(\mathrm{CDCl}_{3}, 470 \mathrm{MHz}\right) \delta: 39.6 \mathrm{ppm}$.

HRMS(+APCI) calc'd for $\mathrm{C}_{8} \mathrm{H}_{7} \mathrm{FO}_{3} \mathrm{~S}\left[\mathrm{M}+\mathrm{H}^{+}\right]$203.0173, found 203.0170.

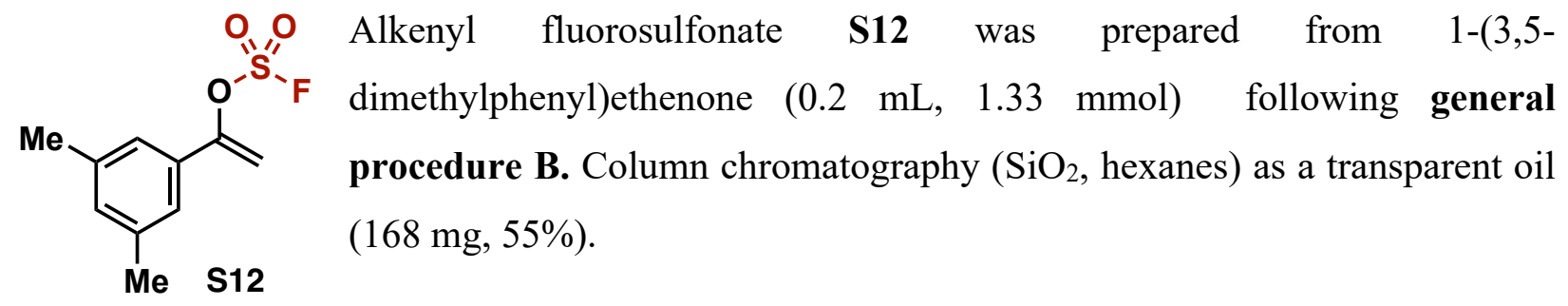

$\mathrm{R}_{\mathrm{f}}=0.74$ (1:4 EtOAc:hexanes)

${ }^{1} \mathrm{H}$ NMR $\left(\mathrm{CDCl}_{3}, 400 \mathrm{MHz}\right) \delta: 7.15$ (s, $\left.2 \mathrm{H}\right), 7.07$ (s, $\left.1 \mathrm{H}\right), 5.61-5.60$ (d, J=4.0 Hz, $\left.1 \mathrm{H}\right), 5.41-$ $5.40(\mathrm{dd}, J=4.0,1.4 \mathrm{~Hz}, 1 \mathrm{H}), 2.35(\mathrm{~s}, 6 \mathrm{H}) \mathrm{ppm}$.

${ }^{13} \mathrm{C} \mathrm{NMR}\left(\mathrm{CDCl}_{3}, 126 \mathrm{MHz}\right) \delta: 154.6,138.7,132.3,131.1,123.3,103.5,21.4 \mathrm{ppm}$.

${ }^{19} \mathrm{~F} \mathrm{NMR}\left(\mathrm{CDCl}_{3}, 470 \mathrm{MHz}\right) \delta: 39.5 \mathrm{ppm}$

HRMS(-APCI) calc'd for $\mathrm{C}_{10} \mathrm{H}_{11} \mathrm{FO}_{3} \mathrm{~S}\left[{\left.\mathrm{M}-\mathrm{H}^{-}\right]}^{229.0329}\right.$, found 229.0336. 


\section{$\underline{\text { Synthesis of Alkenyl Sulfamates: General Procedure C }}$}

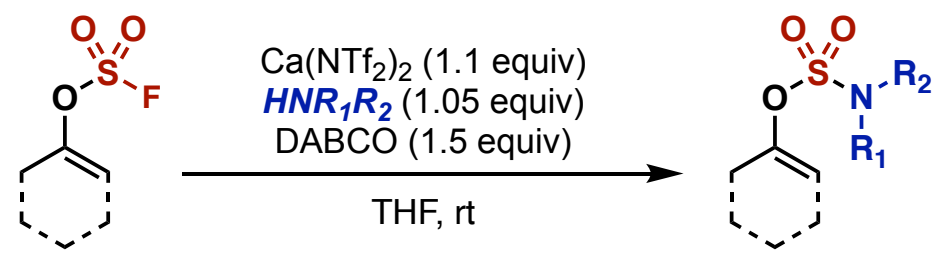

To a flame-dried $25 \mathrm{~mL}$ round-bottom-flask equipped with a stir bar, $\mathrm{Ca}\left(\mathrm{NTf}_{2}\right)_{2}(102 \mathrm{mg}, 0.17$ mmol, 1.10 equiv) and DABCO (26 mg, $0.23 \mathrm{mmol}, 1.50$ equiv) were added and the flask was evacuated 3 times and placed under an atmosphere of nitrogen. The amine substrate $(0.16 \mathrm{mmol}$, 1.05 equiv) was added followed by dry THF $(0.3 \mathrm{~mL}, \mathrm{C}=0.5 \mathrm{M})$. The solution was stirred at room temperature and monitored by TLC. Once full consumption of the starting material was reached (typically $12-48 \mathrm{~h})$, sat. $\mathrm{NH}_{4} \mathrm{Cl}(5 \mathrm{~mL})$ was added and the resulting mixture was extracted using EtOAc $(3 \times 15 \mathrm{~mL})$. The combined organic phases were washed with brine, dried over $\mathrm{MgSO}_{4}$, and the solvent was removed under reduced pressure. Column chromatography afforded the pure alkenyl sulfamate. Note: While the SuFEx procedure leads to almost pure products before purification via column chromatography, full removal of bistriflimide byproducts was found to be crucial to ensure high yields in the photochemistry step. Absence of residual bistriflimide anions can be checked by ${ }^{19} F$ NMR.

$\underline{\text { Synthesis and Characterization of Alkenyl Sulfamates }}$<smiles>O=S(=O)(NCc1ccccc1)OC1=CCCc2ccccc21</smiles>

4

Alkenyl sulfamate 4 was prepared from alkenyl fluorosulfonate 2 (34 $\mathrm{mg}, 0.15 \mathrm{mmol}$ ) and benzylamine following general procedure C (reaction time $=16 \mathrm{~h})$. Column chromatography $\left(\mathrm{SiO}_{2}, 2: 98\right.$ to $5: 95$ EtOAc:hexanes) afforded $\mathbf{4}$ as a colorless oil (38 $\mathrm{mg}, 80 \%$ ).

$\mathrm{R}_{\mathrm{f}}=0.42(1: 4$ EtOAc:hexanes) 
${ }^{1} \mathrm{H}$ NMR $\left(\mathrm{CDCl}_{3}, 400 \mathrm{MHz}\right) \delta: 7.42-7.15(\mathrm{~m}, 9 \mathrm{H}), 6.00-5.97(\mathrm{t}, J=4.8 \mathrm{~Hz}, 1 \mathrm{H}), 4.96-4.93(\mathrm{t}$, $J=6.0 \mathrm{~Hz}, 1 \mathrm{H}), 4.37-4.36(\mathrm{~d}, J=6.0 \mathrm{~Hz}, 2 \mathrm{H}), 2.85-2.81(\mathrm{t}, J=8.1 \mathrm{~Hz}, 2 \mathrm{H}), 2.46-2.41(\mathrm{~m}, 2$ H) ppm.

${ }^{13} \mathrm{C} \mathrm{NMR}\left(\mathrm{CDCl}_{3}, 126 \mathrm{MHz}\right) \delta: 146.0,136.6,135.9,130.1,129.0,128.6,128.4,128.1,127.7$, $126.7,121.7,115.9,48.5,27.3,22.3 \mathrm{ppm}$.

HRMS(+ESI) calc'd for $\mathrm{C}_{17} \mathrm{H}_{17} \mathrm{NO}_{3} \mathrm{~S}\left[\mathrm{M}+\mathrm{H}^{+}\right]$316.1002, found 316.0997.

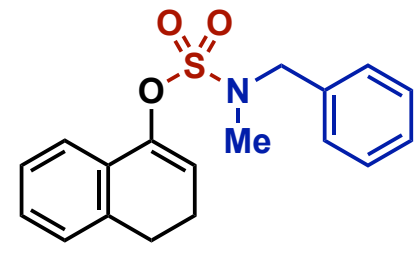

5

Alkenyl sulfamate $\mathbf{5}$ was prepared by starting with alkenyl fluorosulfonate 2 (34 $\mathrm{mg}, 0.15 \mathrm{mmol})$, and $N$-benzylmethylamine following general procedure $\mathbf{C}$ (reaction time $=16 \mathrm{~h}$ ). Column chromatography $\left(\mathrm{SiO}_{2}, 10: 90\right.$ EtOAc:hexanes) afforded $\mathbf{5}$ as a colorless oil (49 mg, 99\%).

$\mathrm{R}_{\mathrm{f}}=0.46(1: 4$ EtOAc:hexanes)

${ }^{1} \mathrm{H}$ NMR $\left(\mathrm{CDCl}_{3}, 400 \mathrm{MHz}\right) \delta: 7.43-7.31(\mathrm{~m}, 6 \mathrm{H}), 7.24-7.15(\mathrm{~m}, 3 \mathrm{H}), 6.07-6.05(\mathrm{t}, J=4.7 \mathrm{~Hz}$, $1 \mathrm{H}), 4.46$ (s, $2 \mathrm{H}), 2.87-2.83$ (m, $5 \mathrm{H}), 2.50-2.44$ (m, $2 \mathrm{H}) \mathrm{ppm}$.

${ }^{13} \mathrm{C} \mathrm{NMR}\left(\mathrm{CDCl}_{3}, 126 \mathrm{MHz}\right) \delta: 145.7,136.6,135.2,130.3,128.9,128.6,128.5,128.3,127.7$, 126.7, 121.6, 116.0, 55.3, 35.6, 27.4, 22.4 ppm.

HRMS(+ESI) calc'd for $\mathrm{C}_{18} \mathrm{H}_{19} \mathrm{NO}_{3} \mathrm{~S}\left[\mathrm{M}+\mathrm{H}^{+}\right] 330.1158$, found 330.1153 .<smiles>CC(NS(=O)(=O)OC1=CCCc2ccccc21)c1ccccc1</smiles>

$\mathrm{R}_{\mathrm{f}}=0.48$ (1:4 EtOAc:hexanes) $\mathrm{Hz}, 1 \mathrm{H}), 5.05-5.04(\mathrm{~d}, J=7.0 \mathrm{~Hz}, 1 \mathrm{H}), 4.77-4.71(\mathrm{p}, J=6.9 \mathrm{~Hz}, 1 \mathrm{H}), 2.82-2.69$ (m, $2 \mathrm{H})$, 2.39-2.34 (td, $J=8.1,4.8 \mathrm{~Hz}, 2 \mathrm{H}), 1.57-1.55$ (d, $J=6.9 \mathrm{~Hz}, 3 \mathrm{H})$ ppm. 
${ }^{13} \mathrm{C}$ NMR $\left(\mathrm{CDCl}_{3}, 126 \mathrm{MHz}\right) \delta: 146.0,141.9,136.5,130.1,129.0,128.4,128.1,127.5,126.7$, $126.2,121.8,114.9,55.2,27.3,23.4,22.3 \mathrm{ppm}$.

HRMS(+ESI): calc'd for $\mathrm{C}_{18} \mathrm{H}_{19} \mathrm{NO}_{3} \mathrm{~S}\left[\mathrm{M}+\mathrm{H}^{+}\right]$330.1157, found 330.1157 .

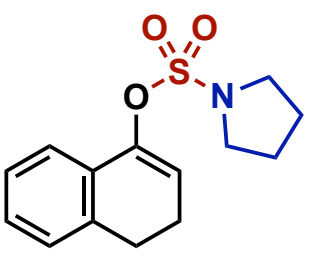

3

Alkenyl sulfamate $\mathbf{3}$ was prepared by starting with alkenyl fluorosulfonate $\mathbf{2}$ (34 $\mathrm{mg}, 0.15 \mathrm{mmol}$ ), and pyrrolidine following general procedure $\mathbf{C}$ (reaction time $=16$ h). Column chromatography $\left(\mathrm{SiO}_{2}, \quad 10: 90\right.$ EtOAc:hexanes) afforded $\mathbf{3}$ as a colorless oil (41 mg, 98\%).

$\mathrm{R}_{\mathrm{f}}=0.40$ (1:4 EtOAc:hexanes)

${ }^{1} \mathrm{H} \mathrm{NMR}\left(\mathrm{CDCl}_{3}, 400 \mathrm{MHz}\right)$ 8: 7.43-7.41 (m, $\left.1 \mathrm{H}\right), 7.24-7.18$ (m, $\left.2 \mathrm{H}\right), 7.17-7.12(\mathrm{~m}, 1 \mathrm{H})$, 6.03-6.02 (t, $J=4.8 \mathrm{~Hz}, 1 \mathrm{H}), 3.52-3.48(\mathrm{~m}, 4 \mathrm{H}), 2.84-2.80$ (t, $J=8.1 \mathrm{~Hz}, 2 \mathrm{H}), 2.47-2.42$ (ddd, $J=9.0,7.5,4.8 \mathrm{~Hz}, 2 \mathrm{H}), 1.94-1.87$ (m, $4 \mathrm{H}$ ) ppm. ${ }^{13} \mathrm{C} \mathrm{NMR}\left(\mathrm{CDCl}_{3}, 126 \mathrm{MHz}\right) \delta: 145.7,136.6,130.5,128.4,127.6,126.6,121.5,115.8,49.4$, 27.4, 25.8, 22.4 ppm.

HRMS(+ESI): calc'd for $\mathrm{C}_{14} \mathrm{H}_{17} \mathrm{NO}_{3} \mathrm{~S}\left[\mathrm{M}+\mathrm{H}^{+}\right]$280.1002, found 280.0999 .

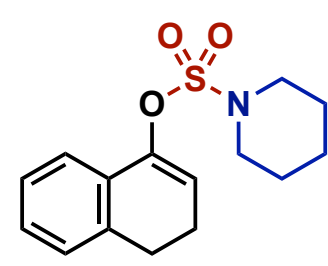

7

Alkenyl sulfamate 7 was prepared by starting with alkenyl fluorosulfonate $\mathbf{2}$ (34 $\mathrm{mg}, 0.15 \mathrm{mmol}$ ), and piperidine following general procedure $\mathbf{C}$ (reaction time $=16$ h). Column chromatography $\left(\mathrm{SiO}_{2}, \quad 10: 90\right.$ EtOAc:hexanes) to afford 7 as a white solid (42 mg, 95\%).

$\mathrm{R}_{\mathrm{f}}=0.56$ (1:4 EtOAc:hexanes)

${ }^{1} \mathrm{H} \mathrm{NMR}\left(\mathrm{CDCl}_{3}, 400 \mathrm{MHz}\right)$ 8: 7.43-7.37 (m, $\left.1 \mathrm{H}\right), 7.24-7.18$ (m, $\left.2 \mathrm{H}\right), 7.16-7.12$ (m, $\left.1 \mathrm{H}\right)$, 6.04-6.02 (t, $J=4.8 \mathrm{~Hz}, 1 \mathrm{H}), 3.43-3.40(\mathrm{~m}, 4 \mathrm{H}), 2.84-2.80$ (t, $J=8.1 \mathrm{~Hz}, 2 \mathrm{H}), 2.47-2.42$ (td, $J=8.1,4.8 \mathrm{~Hz}, 2 \mathrm{H}), 1.70-1.65$ (m, $4 \mathrm{H}), 1.61-1.54$ (m, $2 \mathrm{H}) \mathrm{ppm}$.

${ }^{13} \mathrm{C} \mathrm{NMR}\left(\mathrm{CDCl}_{3}, 126 \mathrm{MHz}\right) \delta: 145.6,136.6,130.5,128.4,127.6,126.6,121.6,115.5,48.2$, 27.4, 25.2, 23.6, $22.4 \mathrm{ppm}$.

HRMS(+ESI): calc'd for $\mathrm{C}_{15} \mathrm{H}_{19} \mathrm{NO}_{3} \mathrm{~S}\left[\mathrm{M}+\mathrm{H}^{+}\right]$294.1158, found 294.1153 .

mp: $64-65^{\circ} \mathrm{C}$ 


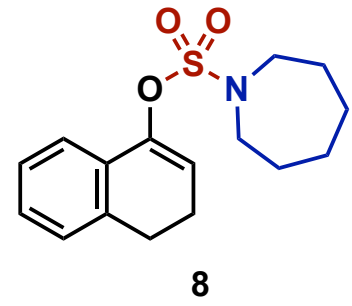

$\mathrm{R}_{\mathrm{f}}=0.56$ (1:4 EtOAc:hexanes)

${ }^{1} \mathrm{H}$ NMR $\left(\mathrm{CDCl}_{3}, 400 \mathrm{MHz}\right) \delta: 7.44-7.40$ (m, $\left.1 \mathrm{H}\right), 7.24-7.18(\mathrm{~m}, 2 \mathrm{H}), 7.16-7.11(\mathrm{~m}, 1 \mathrm{H}), 6.02-$ $6.00(\mathrm{t}, J=4.8 \mathrm{~Hz}, 1 \mathrm{H}), 3.49-3.46(\mathrm{~m}, 4 \mathrm{H}), 2.82-2.80(\mathrm{t}, J=8.1 \mathrm{~Hz}, 2 \mathrm{H}), 2.47-2.41$ (td, $J=8.2$, 4.7 Hz, 2 H), 1.79-1.73 (m, 4 H), 1.66-1.59 (m, 4 H) ppm.

${ }^{13} \mathrm{C} \mathrm{NMR}\left(\mathrm{CDCl}_{3}, 126 \mathrm{MHz}\right) \delta: 145.9,136.6,130.6,128.4,127.6,126.6,121.7,115.9,49.8$, 28.9, 27.5, 27.0, $22.4 \mathrm{ppm}$.

HRMS(+ESI): calc'd for $\mathrm{C}_{16} \mathrm{H}_{21} \mathrm{NO}_{3} \mathrm{~S}\left[\mathrm{M}+\mathrm{H}^{+}\right]$308.1315, found 308.1309.

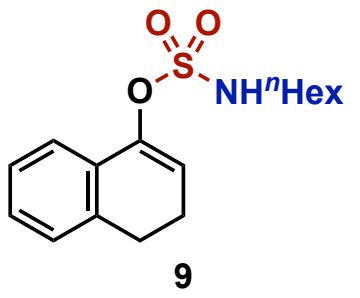

Alkenyl sulfamate 9 was prepared by starting with alkenyl fluorosulfonate 2 (34 mg, $0.15 \mathrm{mmol}$ ), and hexylamine following general procedure C (reaction time $=16 \mathrm{~h})$. Column chromatography $\left(\mathrm{SiO}_{2}\right.$, hexanes to $10: 90$ EtOAc:hexanes) afforded 9 as a pale-yellow oil (43 mg, 93\%).

$\mathrm{R}_{\mathrm{f}}=0.81$ (1:4 EtOAc:hexanes)

${ }^{1} \mathrm{H} \mathrm{NMR}\left(\mathrm{CDCl}_{3}, 400 \mathrm{MHz}\right) \delta: 7.44-7.42$ (m, $\left.1 \mathrm{H}\right), 7.25-7.18(\mathrm{~m}, 2 \mathrm{H}), 7.15-7.13$ (m, $\left.1 \mathrm{H}\right), 6.01-$ $5.99(\mathrm{t}, J=4.8 \mathrm{~Hz}, 1 \mathrm{H}), 4.73-4.70(\mathrm{t}, J=5.9 \mathrm{~Hz}, 1 \mathrm{H}), 3.23-3.18(\mathrm{q}, J=6.7 \mathrm{~Hz}, 2 \mathrm{H}), 2.85-2.81$ (t, $J=8.1 \mathrm{~Hz}, 2 \mathrm{H}), 2.48-2.43(\mathrm{td}, J=8.1,4.7 \mathrm{~Hz}, 2 \mathrm{H}), 1.54-1.47$ (p, $J=7.1 \mathrm{~Hz}, 2 \mathrm{H}), 1.32-1.20$ (m, $6 \mathrm{H}), 0.89-0.85(\mathrm{t}, J=6.7 \mathrm{~Hz}, 3 \mathrm{H}) \mathrm{ppm}$.

${ }^{13} \mathrm{C} \mathrm{NMR}\left(\mathrm{CDCl}_{3}, 126 \mathrm{MHz}\right) \delta: 146.0,136.5,130.2,128.5,127.7,126.7,121.7,115.7,44.6,31.4$, 29.6, 27.3, 26.2, 22.6, 22.4, $14.1 \mathrm{ppm}$.

HRMS(+ESI): calc'd for $\mathrm{C}_{16} \mathrm{H}_{24} \mathrm{NO}_{3} \mathrm{~S}[\mathrm{M}+\mathrm{H}]^{+} 310.1471$, found 310.1467 .

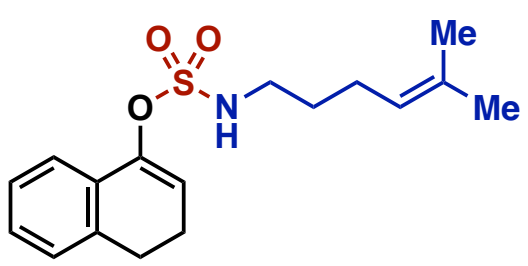

10

Alkenyl sulfamate $\mathbf{1 0}$ was prepared by starting with alkenyl fluorosulfonate 2 (34 mg, $0.15 \mathrm{mmol}$ ), and 5-Methyl-4-hexen-1amine (S4) following general procedure $\mathbf{C}$ (reaction time $=16$ h). Column chromatography $\left(\mathrm{SiO}_{2}\right.$, hexanes to 5:95 EtOAc:hexanes afforded 10 as a brown oil (32 mg, 67\%). 
$\mathrm{R}_{\mathrm{f}}=0.62(1: 4$ EtOAc:hexanes)

${ }^{1} \mathrm{H}$ NMR $\left(\mathrm{CDCl}_{3}, 400 \mathrm{MHz}\right) \delta: 7.45-7.41$ (m, $\left.1 \mathrm{H}\right), 7.24-7.18(\mathrm{~m}, 2 \mathrm{H}), 7.16-7.12$ (m, $\left.1 \mathrm{H}\right), 6.01-$ $5.99(\mathrm{t}, J=4.7 \mathrm{~Hz}, 1 \mathrm{H}), 5.05-5.01(\mathrm{tt}, J=7.2,1.4 \mathrm{~Hz}, 1 \mathrm{H}), 4.83-4.80(\mathrm{t}, J=6.0 \mathrm{~Hz}, 1 \mathrm{H}), 3.23-$ $3.18(\mathrm{~m}, 2 \mathrm{H}), 2.85-2.81(\mathrm{t}, J=8.1 \mathrm{~Hz}, 2 \mathrm{H}), 2.48-2.42(\mathrm{td}, J=8.2,4.8 \mathrm{~Hz}, 2 \mathrm{H}), 2.03-1.97(\mathrm{q}, J$ $=7.3 \mathrm{~Hz}, 2 \mathrm{H}), 1.68(\mathrm{~s}, 3 \mathrm{H}), 1.58(\mathrm{~s}, 3 \mathrm{H}) 1.61-1.52(\mathrm{~m}, 2 \mathrm{H}) \mathrm{ppm}$.

${ }^{13} \mathrm{C} \mathrm{NMR}\left(\mathrm{CDCl}_{3}, 126 \mathrm{MHz}\right) \delta: 146.0,136.6,133.2,130.2,128.5,127.6,126.7,122.9,121.7$, $115.7,44.3,29.6,27.3,25.8,25.1,22.4,17.8 \mathrm{ppm}$.

HRMS(+APCI): calc'd for $\mathrm{C}_{17} \mathrm{H}_{24} \mathrm{NO}_{3} \mathrm{~S}[\mathrm{M}+\mathrm{H}]^{+} 322.1471$, found 322.1466.

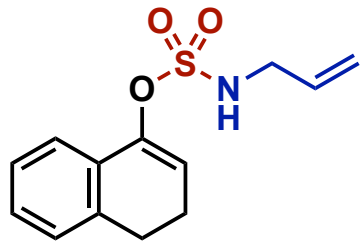

11

Alkenyl sulfamate $\mathbf{1 1}$ was prepared by starting with alkenyl fluorosulfonate 2 (34 $\mathrm{mg}, 0.15 \mathrm{mmol})$, and allylamine following general procedure $\mathbf{C}$ (reaction time $=24 \mathrm{~h}$ ). Note: This reaction was set in a nitrogen-filled glove box due to the air sensitivity of allylamine). Column chromatography $\left(\mathrm{SiO}_{2}, 2: 98\right.$ to 5:95 EtOAc:hexanes) afforded 11 as an off-white solid (31 mg, 77\%).

$\mathrm{R}_{\mathrm{f}}=0.33$ (1:4 EtOAc:hexanes)

${ }^{1} \mathrm{H}$ NMR $\left(\mathrm{CDCl}_{3}, 400 \mathrm{MHz}\right) \delta: 7.45-7.41$ (m, $\left.1 \mathrm{H}\right), 7.25-7.19$ (m, $\left.2 \mathrm{H}\right), 7.16-7.14$ (m, $\left.1 \mathrm{H}\right), 6.03-$ $6.01(\mathrm{t}, J=4.7 \mathrm{~Hz}, 1 \mathrm{H}), 5.88-5.79$ (ddt, $J=17.2,10.2,5.9 \mathrm{~Hz}, 1 \mathrm{H}), 5.30-5.18(\mathrm{~m}, 2 \mathrm{H}), 4.71-$ $4.68(\mathrm{t}, J=6.1 \mathrm{~Hz}, 1 \mathrm{H}), 3.86-3.82(\mathrm{tt}, J=6.0,1.5 \mathrm{~Hz}, 2 \mathrm{H}), 2.86-2.82$ (t, $J=8.1 \mathrm{~Hz}, 2 \mathrm{H}), 2.49-$ $2.43(\mathrm{td}, J=8.1,4.8 \mathrm{~Hz}, 2 \mathrm{H}) \mathrm{ppm}$.

NMR $\left(\mathrm{CDCl}_{3}, 126 \mathrm{MHz}\right) \delta: 146.1,136.6,132.6,130.2,128.6,127.7,126.7,121.7,118.6,115.9$, 47.0, 27.4, $22.4 \mathrm{ppm}$.

HRMS(+ESI): calc'd for $\mathrm{C}_{13} \mathrm{H}_{15} \mathrm{NO}_{3} \mathrm{~S}[\mathrm{M}+\mathrm{H}]^{+} 266.0845$ found 266.0842.

mp: $37-38{ }^{\circ} \mathrm{C}$

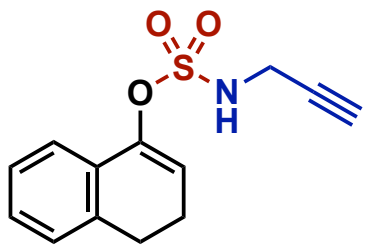

Alkenyl sulfamate $\mathbf{1 2}$ was prepared by starting with alkenyl fluorosulfonate 2 (34 $\mathrm{mg}, 0.15 \mathrm{mmol}$ ), and propargylamine following general procedure $\mathbf{C}$ (reaction time $=48 \mathrm{~h}$ ). Column chromatography

12 
$\left(\mathrm{SiO}_{2}, 2: 98\right.$ to 5:95 EtOAc:hexanes) afforded 12 as an off-white solid (27 mg, 68\%).

$\mathrm{R}_{\mathrm{f}}=0.33$ (1:4 EtOAc:hexanes)

${ }^{1} \mathrm{H}$ NMR $\left(\mathrm{CDCl}_{3}, 400 \mathrm{MHz}\right) \delta: 7.46-7.44(\mathrm{~m}, 1 \mathrm{H}), 7.25-7.13(\mathrm{~m}, 3 \mathrm{H}), 6.07-6.05(\mathrm{t}, J=4.7 \mathrm{~Hz}$, $1 \mathrm{H}), 5.12-5.09$ (t, $J=5.8 \mathrm{~Hz}, 1 \mathrm{H}), 4.02-4.00(\mathrm{dd}, J=5.8,2.5 \mathrm{~Hz}, 2 \mathrm{H}), 2.86-2.82(\mathrm{t}, J=8.2 \mathrm{~Hz}$, $2 \mathrm{H}), 2.49-2.43(\mathrm{td}, J=8.2,4.7 \mathrm{~Hz}, 2 \mathrm{H}), 2.33-2.32(\mathrm{t}, J=2.5 \mathrm{~Hz}, 1 \mathrm{H}) \mathrm{ppm}$.

${ }^{13} \mathrm{C} \mathrm{NMR}\left(\mathrm{CDCl}_{3}, 126 \mathrm{MHz}\right) \delta: 146.0,136.5,130.0,128.6,127.6,126.7,121.8,116.1,77.8,73.9$, 34.0, 27.2, $22.3 \mathrm{ppm}$.

HRMS(+ESI): calc'd for $\mathrm{C}_{13} \mathrm{H}_{13} \mathrm{NO}_{3} \mathrm{~S}[\mathrm{M}+\mathrm{H}]^{+} 264.0689$ found 264.0685 .

mp: $51-52{ }^{\circ} \mathrm{C}$

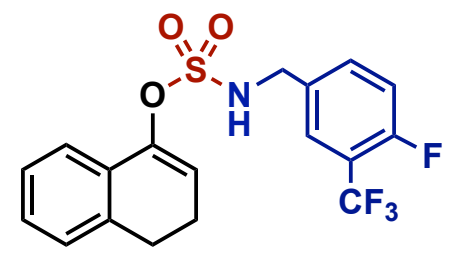

13
Alkenyl sulfamate $\mathbf{1 3}$ was prepared by starting with alkenyl fluorosulfonate 2 (34 $\mathrm{mg}, \quad 0.15 \mathrm{mmol}$ ), and 4-fluoro-3(trifluoromethyl)benzylamine following general procedure $C$. Column chromatography ( $\mathrm{SiO}_{2}, 10: 90$ EtOAc:hexanes) afforded 13 as a white solid (46 mg, 77\%).

$\mathrm{R}_{\mathrm{f}}=0.13$ (1:9 EtOAc:hexanes)

${ }^{1} \mathrm{H}$ NMR $\left(\mathrm{CDCl}_{3}, 400 \mathrm{MHz}\right) \delta:$ 7.48-7.44 (m, $\left.2 \mathrm{H}\right), 7.36-7.32$ (m, $\left.1 \mathrm{H}\right), 7.25-7.18$ (m, $\left.2 \mathrm{H}\right), 7.17-$ $7.12(\mathrm{~m}, 2 \mathrm{H}), 6.01-5.99$ (t, $J=4.7 \mathrm{~Hz}, 1 \mathrm{H}), 5.13-5.10$ (t, $J=6.2 \mathrm{~Hz}, 1 \mathrm{H}), 4.37-4.26(\mathrm{~d}, J=6.2$ $\mathrm{Hz}, 2 \mathrm{H}), 2.84-2.80$ (t, $J=8.1 \mathrm{~Hz}, 1 \mathrm{H}), 2.47-2.42(\mathrm{ddd}, J=9.0,7.5,4.8 \mathrm{~Hz}, 2 \mathrm{H}) \mathrm{ppm}$.

${ }^{13} \mathrm{C} \mathrm{NMR}\left(\mathrm{CDCl}_{3}, 126 \mathrm{MHz}\right) \delta: 160.6(\mathrm{~d}, J=1.9 \mathrm{~Hz}), 158.6-158.5(\mathrm{~d}, J=2.0 \mathrm{~Hz}), 146.0,136.6$, $133.7-133.6(\mathrm{~d}, J=8.7 \mathrm{~Hz}), 132.5-132.4(\mathrm{~d}, J=3.9 \mathrm{~Hz}), 129.9,128.8,127.8,126.9-126.8(\mathrm{qd}, J$ $=4.6,1.8 \mathrm{~Hz}), 126.7,125.7-119.2$ (q, $J=272.5 \mathrm{~Hz}), 121.5,119.3-118.4(\mathrm{qd}, J=33.2,12.8 \mathrm{~Hz})$, $117.7,117.5,116.1,47.3,27.2,22.3 \mathrm{ppm}$.

${ }^{19} \mathrm{~F} \mathrm{NMR}\left(\mathrm{CDCl}_{3}, 470 \mathrm{MHz}\right) \delta:-61.5(\mathrm{~d}, J=12.7 \mathrm{~Hz}),-114.7 \mathrm{ppm}$.

HRMS(+APCI): calc'd for $\mathrm{C}_{18} \mathrm{H}_{15} \mathrm{~F}_{4} \mathrm{NO}_{3} \mathrm{~S}[\mathrm{M}+\mathrm{H}]^{+} 402.0782$, found 402.0773 .

mp: $53-55^{\circ} \mathrm{C}$ 


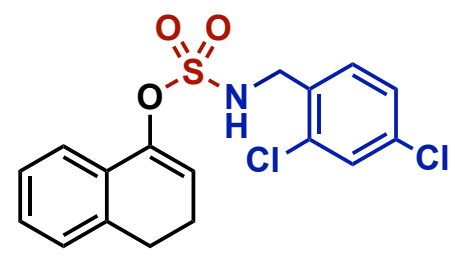

14

Alkenyl sulfamate $\mathbf{1 4}$ was prepared by starting with alkenyl fluorosulfonate 2 (34 mg, $0.15 \mathrm{mmol}$ ), and 2,4-dichlorobenzylamine following general procedure $\mathbf{C}$ (reaction time $=24 \mathrm{~h}$ ). Column chromatography ( $\mathrm{SiO}_{2}, 2: 98$ to 5:95 EtOAc:hexanes) afforded 14 as a colorless oil (36 mg, 62\%).

$\mathrm{R}_{\mathrm{f}}=0.44$ (1:4 EtOAc:hexanes)

${ }^{1} \mathrm{H} \mathrm{NMR}\left(\mathrm{CDCl}_{3}, 400 \mathrm{MHz}\right) \delta: \delta 7.34-7.26(\mathrm{~m}, 3 \mathrm{H}), 7.23-7.12(\mathrm{~m}, 4 \mathrm{H}), 5.97-5.94(\mathrm{t}, J=4.7$ $\mathrm{Hz}, 1 \mathrm{H}), 5.25-5.21(\mathrm{t}, J=6.5 \mathrm{~Hz}, 1 \mathrm{H}), 4.43-4.42(\mathrm{~d}, J=6.4 \mathrm{~Hz}, 2 \mathrm{H}), 2.80(\mathrm{t}, J=8.1 \mathrm{~Hz}, 2 \mathrm{H})$, 2.45-2.40 (td, $J=8.2,4.8 \mathrm{~Hz}, 2 \mathrm{H}) \mathrm{ppm}$.

${ }^{13} \mathrm{C} \mathrm{NMR}\left(\mathrm{CDCl}_{3}, 126 \mathrm{MHz}\right) \delta: 146.0,136.5,135.2,134.3,132.4,131.5,129.9,129.6,128.7$, $127.8,127.7,126.7,121.6,115.2,45.8,27.3,22.4$ ppm.

HRMS(+ESI): calc'd for $\mathrm{C}_{17} \mathrm{H}_{15} \mathrm{Cl}_{2} \mathrm{NO}_{3} \mathrm{~S}[\mathrm{M}+\mathrm{H}]^{+} 384.0222$ found 384.0214 .

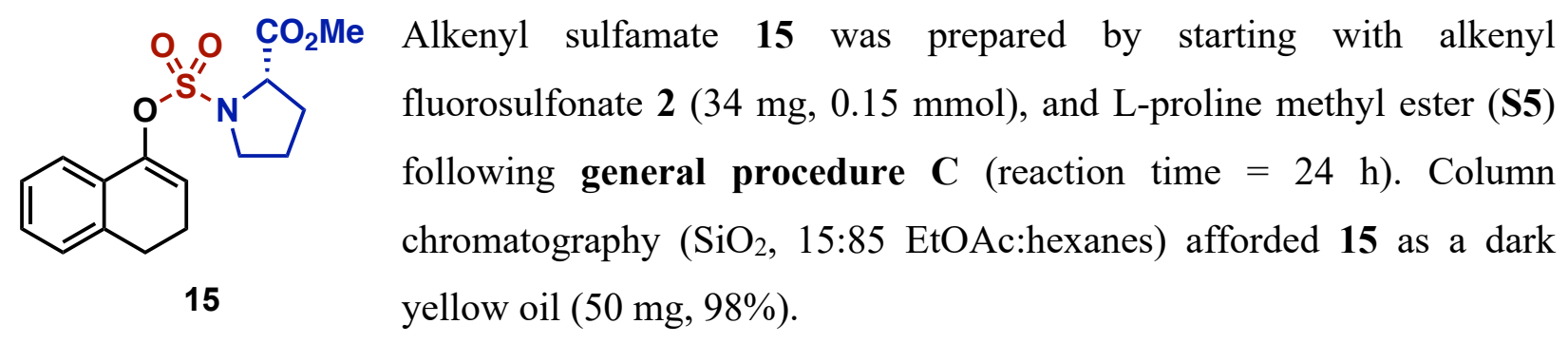

$\mathrm{R}_{\mathrm{f}}=0.22(1: 4$ EtOAc:hexanes)

${ }^{1} \mathrm{H}$ NMR $\left(\mathrm{CDCl}_{3}, 400 \mathrm{MHz}\right) \delta: 7.43-7.40$ (m, $\left.1 \mathrm{H}\right), 7.25-7.18(\mathrm{~m}, 2 \mathrm{H}), 7.17-7.12$ (m, $\left.1 \mathrm{H}\right), 6.07-$ $6.05(\mathrm{t}, J=4.8 \mathrm{~Hz}, 1 \mathrm{H}), 4.49-4.46(\mathrm{dd}, J=8.2,3.4 \mathrm{~Hz}, 1 \mathrm{H}), 3.75$ (s, $3 \mathrm{H}), 3.72-3.66(\mathrm{~m}, 1 \mathrm{H})$, 3.65-3.56 (m, $1 \mathrm{H}), 2.84-2.80(\mathrm{t}, J=8.1 \mathrm{~Hz}, 2 \mathrm{H}), 2.47-2.42$ (ddd, $J=9.1,7.4,4.8 \mathrm{~Hz}, 2 \mathrm{H})$, 2.20-1.98 (m, $3 \mathrm{H}), 1.96-1.88(\mathrm{~m}, 1 \mathrm{H}) \mathrm{ppm}$.

${ }^{13} \mathrm{C} \mathrm{NMR}\left(\mathrm{CDCl}_{3}, 126 \mathrm{MHz}\right) \delta: 172.0,145.8,136.6,130.3,128.5,127.6,126.6,121.5,116.1$, 61.9, 52.6, 50.0, 31.1, 27.4, 24.6, 22.4 ppm.

HRMS(+APCI): calc'd for $\mathrm{C}_{16} \mathrm{H}_{19} \mathrm{NO}_{5} \mathrm{~S}[\mathrm{M}+\mathrm{H}]^{+} 338.1057$, found 336.1050 . 
(1)

Alkenyl sulfamate $\mathbf{1 6}$ was prepared by starting with alkenyl fluorosulfonate $\mathbf{S 1 0}$ (39 $\mathrm{mg}, 0.15 \mathrm{mmol}$ ), and piperidine following general procedure $\mathbf{C}$ (reaction time $=12 \mathrm{~h}$ ). Column chromatography $\left(\mathrm{SiO}_{2}\right.$, hexanes to 10:90 EtOAc:hexanes) afforded $\mathbf{1 6}$ as a light brown oil (37 mg, 77\%).

$\mathrm{R}_{\mathrm{f}}=0.63$ (1:2 EtOAc:hexanes)

${ }^{1} \mathrm{H}$ NMR $\left(\mathrm{CDCl}_{3}, 400 \mathrm{MHz}\right) \delta: 7.06-7.04(\mathrm{~d}, J=8.2 \mathrm{~Hz}, 1 \mathrm{H}), 6.98(\mathrm{~d}, J=2.7 \mathrm{~Hz}, 1 \mathrm{H}), 6.76-$ $6.73(\mathrm{dd}, J=8.2,2.7 \mathrm{~Hz}, 1 \mathrm{H}), 6.06-6.03(\mathrm{t}, J=4.8 \mathrm{~Hz}, 1 \mathrm{H}), 3.80(\mathrm{~s}, 3 \mathrm{H}), 3.43-3.40(\mathrm{~m}, 4 \mathrm{H})$, $2.77-2.73$ (t, $J=8.1 \mathrm{~Hz}, 2 \mathrm{H}), 2.45-2.39$ (td, $J=8.1,4.8 \mathrm{~Hz}, 2 \mathrm{H}), 1.70-1.65$ (m, $J=5.7 \mathrm{~Hz}, 4 \mathrm{H})$, $1.60-1.54$ (m, $2 \mathrm{H}) \mathrm{ppm}$.

${ }^{13} \mathrm{C} \mathrm{NMR}\left(\mathrm{CDCl}_{3}, 126 \mathrm{MHz}\right) \delta: \delta 158.4,145.5,131.4,128.7,128.4,116.2,113.2,107.9,55.4$, 48.1, 26.5, 25.2, 23.6, $22.7 \mathrm{ppm}$.

HRMS(+ESI): calc'd for $\mathrm{C}_{16} \mathrm{H}_{21} \mathrm{NO}_{4} \mathrm{~S}[\mathrm{M}+\mathrm{H}]^{+} 324.1264$, found 324.1260.

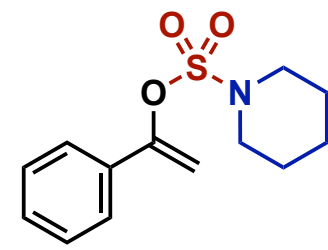

17

Alkenyl sulfamate 17 was prepared by starting with alkenyl fluorosulfonate $\mathbf{S 1 1}$ (30 $\mathrm{mg}, 0.15 \mathrm{mmol}$ ), and piperidine following general procedure C (reaction time $=24 \mathrm{~h})$. Column chromatography $\left(\mathrm{SiO}_{2}, 2: 98\right.$ EtOAc:hexanes) afforded 17 as a colorless oil (40 mg, >99\%).

$\mathrm{R}_{\mathrm{f}}=0.45$ (1:4 EtOAc:hexanes)

${ }^{1} \mathrm{H}$ NMR $\left(\mathrm{CDCl}_{3}, 400 \mathrm{MHz}\right) \delta: \delta$ 7.59-7.55 (m, $\left.2 \mathrm{H}\right), 7.40-7.35$ (m, $\left.3 \mathrm{H}\right), 5.43-5.42(\mathrm{~d}, J=2.9$ $\mathrm{Hz}, 1 \mathrm{H}), 5.39-5.38$ (d, $J=2.8 \mathrm{~Hz}, 1 \mathrm{H}), 3.39-3.36$ (m, 4 H), 1.69-1.63 (m, $4 \mathrm{H}), 1.58-1.54$ (m, 2 H) $\mathrm{ppm}$.

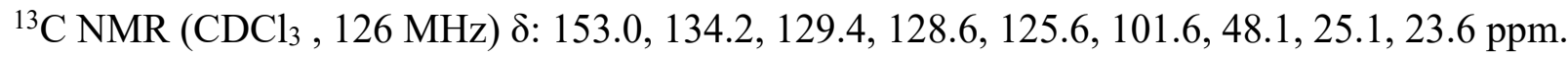
HRMS(+ESI): calc'd for $\mathrm{C}_{13} \mathrm{H}_{17} \mathrm{NO}_{3} \mathrm{~S}\left[\mathrm{M}+\mathrm{H}^{+}\right]$268.1002, found 268.0997 .<smiles>C=C(OS(=O)(=O)N1CCCCC1)c1cc([N+](=O)[O-])cc([N+](=O)[O-])c1</smiles>

Alkenyl sulfamate $\mathbf{1 8}$ was prepared by starting with alkenyl fluorosulfonate $\mathbf{S 1 2}$ (35 $\mathrm{mg}, 0.15 \mathrm{mmol}$ ), and piperidine following general procedure $\mathbf{C}$ (reaction time $=24 \mathrm{~h}$ ). Column chromatography 
$\left(\mathrm{SiO}_{2}, 2: 98\right.$ EtOAc:hexanes) afforded $\mathbf{1 8}$ as a colorless oil (44 mg, $\left.>99 \%\right)$.

$\mathrm{R}_{\mathrm{f}}=0.41$ (1:4 EtOAc:hexanes)

${ }^{1} \mathrm{H} \mathrm{NMR}\left(\mathrm{CDCl}_{3}, 400 \mathrm{MHz}\right) \delta$ : 7.17 (br.s., $2 \mathrm{H}$ ), 7.00 (br. s, $J=2.1 \mathrm{~Hz}, 1 \mathrm{H}$ ), 5.38-5.37 (d, $J=$ $2.8 \mathrm{~Hz}, 1 \mathrm{H}), 5.33-5.32$ (d, $J=2.7 \mathrm{~Hz}, 1 \mathrm{H}), 3.38-3.35$ (m, $4 \mathrm{H}), 2.33$ (s, $6 \mathrm{H}), 1.68-1.63$ (m, 4 $\mathrm{H}), 1.60-1.52$ ( $\mathrm{m}, 2 \mathrm{H})$.

${ }^{13} \mathrm{C} \mathrm{NMR}\left(\mathrm{CDCl}_{3}, 126 \mathrm{MHz}\right) \delta: 153.3,138.1,134.2,131.2,123.5,101.3,48.1,25.2,23.6,21.5$ ppm.

HRMS(+ESI): calc'd for $\mathrm{C}_{15} \mathrm{H}_{21} \mathrm{NO}_{3} \mathrm{~S}\left[\mathrm{M}+\mathrm{H}^{+}\right] 296.1315$ found 296.1309 .

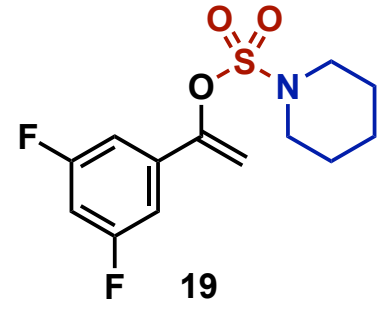

General procedure A was used with 3,5-difluoroacetophenone $(23 \mathrm{mg}$, $0.15 \mathrm{mmol}$ ) and anhydrous $\mathrm{Et}_{2} \mathrm{O}$ at $-78{ }^{\circ} \mathrm{C}$ for $1.5 \mathrm{~h}$. After work-up, solvents were removed under reduced pressure at or below $20^{\circ} \mathrm{C}$ due to the high volatility of the product. The resulting alkenyl fluorosulfonate was prone to decomposition during column chromatography and was therefore used in the next step without further purification: ${ }^{1} \mathrm{H}$ NMR chemical shifts of this intermediate are indicated: ${ }^{1} \mathrm{H} N \mathrm{NM}\left(\mathrm{CDCl}_{3}, 400 \mathrm{MHz}\right) \delta: \delta 7.12-7.05(\mathrm{~m}, 2 \mathrm{H}), 6.89(\mathrm{tt}, J=8.6$, $2.3 \mathrm{~Hz}, 1 \mathrm{H}), 5.72(\mathrm{~d}, J=4.5 \mathrm{~Hz}, 1 \mathrm{H}), 5.59(\mathrm{dd}, J=4.5,1.4 \mathrm{~Hz}, 1 \mathrm{H}) \mathrm{ppm}$. Alkenyl sulfamate 19 was prepared following general procedure $\mathbf{C}$ (reaction time $=24 \mathrm{~h}$ ). Column chromatography $\left(\mathrm{SiO}_{2}, 2: 98\right.$ EtOAc:hexanes) afforded 19 as a colorless oil (20 mg, 45\%).

$\mathrm{R}_{\mathrm{f}}=0.60$ (1:4 EtOAc:hexanes)

${ }^{1} \mathrm{H} \mathrm{NMR}\left(\mathrm{CDCl}_{3}, 400 \mathrm{MHz}\right) \delta$ : 7.11-7.04 (m, $\left.2 \mathrm{H}\right), 6.84-6.78$ (tt, $\left.J=8.7,2.3 \mathrm{~Hz}, 1 \mathrm{H}\right), 5.47$ (s, $1 \mathrm{H}), 3.42-3.39$ (m, $4 \mathrm{H}), 1.72-1.67$ (m, $4 \mathrm{H}), 1.63-1.56$ (m, 2 H) ppm.

${ }^{13} \mathrm{C} \mathrm{NMR}\left(\mathrm{CDCl}_{3}, 126 \mathrm{MHz}\right) \delta: 164.4-164.3(\mathrm{~d}, J=12.8 \mathrm{~Hz}), 161.9-161.8(\mathrm{~d}, J=12.8 \mathrm{~Hz})$, $150.7-150.6$ (t, $J=3.4 \mathrm{~Hz}), 137.7-137.5$ (t, $J=9.8 \mathrm{~Hz}), 108.8-108.7$ (d, $J=27.1 \mathrm{~Hz}), 108.6-$ 108.5 (d, $J=11.6 \mathrm{~Hz}), 105.0-104.5(\mathrm{t}, J=25.5 \mathrm{~Hz}), 103.7,48.1,25.1,23.5 \mathrm{ppm}$.

${ }^{19} \mathrm{~F} \mathrm{NMR}\left(\mathrm{CDCl}_{3}, 470 \mathrm{MHz}\right) \delta:-109.3(\mathrm{~m}) \mathrm{ppm}$.

HRMS(+ESI): calc'd for $\mathrm{C}_{13} \mathrm{H}_{15} \mathrm{~F}_{2} \mathrm{NO}_{3} \mathrm{~S}\left[\mathrm{M}+\mathrm{H}^{+}\right] 304.0813$ found 304.0809 . 


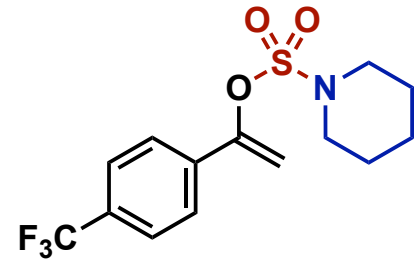

20

General procedure A was used with 4-(trifluoromethyl)acetophenone $(28 \mathrm{mg}, 0.15 \mathrm{mmol})$ and anhydrous $\mathrm{Et}_{2} \mathrm{O}$ at $-78{ }^{\circ} \mathrm{C}$ for $1.5 \mathrm{~h}$. The resulting alkenyl fluorosulfonate was prone to decomposition during column chromatography and was therefore used in the next step without further purification: ${ }^{1} \mathrm{H} \mathrm{NMR}\left(\mathrm{CDCl}_{3}, 400 \mathrm{MHz}\right) \delta: 7.58-7.54(\mathrm{~m}, 2$ H), 7.45-7.42 (m, $2 \mathrm{H}), 5.66(\mathrm{dd}, J=4.0,0.7 \mathrm{~Hz}, 1 \mathrm{H}), 5.46(\mathrm{dd}, J=4.1,1.4 \mathrm{~Hz}, 1 \mathrm{H}) \mathrm{ppm}$. Alkenyl sulfamate 20 was prepared following general procedure $\mathbf{C}$ (reaction time $=24 \mathrm{~h}$ ). Column chromatography ( $\mathrm{SiO}_{2}, 2: 98$ EtOAc:hexanes) afforded 20 as a colorless oil (25 mg, 50\%).

$\mathrm{R}_{\mathrm{f}}=0.53$ (1:4 EtOAc:hexanes)

${ }^{1} \mathrm{H} \mathrm{NMR}\left(\mathrm{CDCl}_{3}, 400 \mathrm{MHz}\right) \delta: 7.69-7.62(\mathrm{q}, J=8.6 \mathrm{~Hz}, 4 \mathrm{H}), 5.53(\mathrm{~d}, J=3.1 \mathrm{~Hz}, 1 \mathrm{H}), 5.49-$ $5.48(\mathrm{~d}, J=3.1 \mathrm{~Hz}, 1 \mathrm{H}), 3.41-3.39$ (m, 4 H), 1.72-1.66 (m, 4 H), $1.61-1.56$ (m, 2 H) ppm. ${ }^{13} \mathrm{C} \mathrm{NMR}\left(\mathrm{CDCl}_{3}, 126 \mathrm{MHz}\right) \delta: 151.7,137.7,131.7-130.9(\mathrm{q}, J=32.7 \mathrm{~Hz}), 125.9,125.7-125.6$ (q, $J=3.8 \mathrm{~Hz}) .127 .3-120.8$ (q, $J=272.1 \mathrm{~Hz}), 103.7,48.2,25.1,23.6 \mathrm{ppm}$.

${ }^{19} \mathrm{~F} \mathrm{NMR}\left(\mathrm{CDCl}_{3}, 470 \mathrm{MHz}\right) \delta:-62.8 \mathrm{ppm}$. HRMS(+ESI): calc'd for $\mathrm{C}_{14} \mathrm{H}_{16} \mathrm{~F}_{3} \mathrm{NO}_{3} \mathrm{~S}\left[\mathrm{M}+\mathrm{H}^{+}\right] 336.0876$ found 336.0871 .

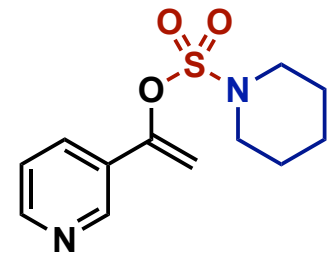

21

General procedure A was used with 3-acetylpyridine (33 mg, $0.16 \mathrm{mmol}$ ) and anhydrous DCM at $0{ }^{\circ} \mathrm{C}$ for $1.5 \mathrm{~h}$. The resulting alkenyl fluorosulfonate was prone to decomposition during column chromatography and was therefore used in the next step without further purification: ${ }^{1} \mathrm{H} \mathrm{NMR}\left(\mathrm{CDCl}_{3}\right.$, $400 \mathrm{MHz}) \delta: 8.82(\mathrm{dd}, J=2.5,0.9 \mathrm{~Hz}, 1 \mathrm{H}), 8.66(\mathrm{dd}, J=4.9,1.6 \mathrm{~Hz}, 1 \mathrm{H})$, $7.83(\mathrm{ddd}, J=8.1,2.5,1.6 \mathrm{~Hz}, 1 \mathrm{H}), 7.37$ (ddd, $J=8.1,4.9,0.9 \mathrm{~Hz}, 1 \mathrm{H}), 5.74$ (dd, $J=4.4,0.7$ $\mathrm{Hz}, 1 \mathrm{H}), 5.57(\mathrm{dd}, J=4.4,1.4 \mathrm{~Hz}, 1 \mathrm{H}) \mathrm{ppm}$. Alkenyl sulfamate 21 was prepared following general procedure $\mathbf{C}$ (reaction time $=16 \mathrm{~h}$ ). Column chromatography $\left(\mathrm{SiO}_{2}, 20: 80\right.$ EtOAc:hexanes) and then preparatory TLC (30:70 EtOAc:hexanes) afforded 21 as a viscous oil (34 mg, 78\%). Note: 21 rearranges to $\beta$-ketosulfonamide 46 over time during chromatography and therefore should be purified rapidly. Less than $10 \%$ of 46 was observed when the purification time was below $20 \mathrm{~min}$.

$\mathrm{R}_{\mathrm{f}}=0.20(2: 3$ EtOAc:hexanes $)$ 
${ }^{1} \mathrm{H}$ NMR $\left(\mathrm{CDCl}_{3}, 400 \mathrm{MHz}\right) \delta: 8.82-8.81(\mathrm{~m}, 1 \mathrm{H}), 8.59-8.58(\mathrm{dd}, J=4.9,1.6 \mathrm{~Hz}, 1 \mathrm{H}), 7.86-$ $7.83(\mathrm{ddd}, J=8.0,2.4,1.6 \mathrm{~Hz}, 1 \mathrm{H}), 7.33-7.29(\mathrm{ddd}, J=8.0,4.9,0.9 \mathrm{~Hz}, 1 \mathrm{H}), 5.49-5.48(\mathrm{~d}, J=$ $3.1 \mathrm{~Hz}, 1 \mathrm{H}), 5.47-5.46(\mathrm{~d}, J=3.1 \mathrm{~Hz}, 1 \mathrm{H}), 3.41-3.38$ (m, $4 \mathrm{H}), 1.70-1.65(\mathrm{~m}, 4 \mathrm{H}), 1.62-1.54$ $(\mathrm{m}, 2 \mathrm{H}) \mathrm{ppm}$.

${ }^{13} \mathrm{C} \mathrm{NMR}\left(\mathrm{CDCl}_{3}, 126 \mathrm{MHz}\right) \delta: 150.5,150.3,1476.9,133.0,130.3,123.4,103.2,48.1,25.1,23.5$ ppm.

HRMS(+APCI): calc'd for $\mathrm{C}_{12} \mathrm{H}_{16} \mathrm{~N}_{2} \mathrm{O}_{3} \mathrm{~S}[\mathrm{M}+\mathrm{H}]^{+}$269.0954, found 269.0948.

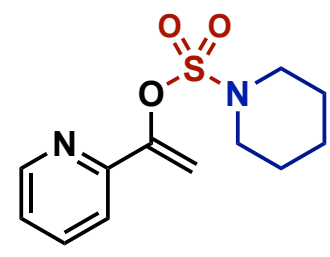

22

General procedure A was used with 2-acetylpyridine (150 mg, $1.24 \mathrm{mmol}$ ) and anhydrous DCM at $0{ }^{\circ} \mathrm{C}$ for $1.5 \mathrm{~h}$. The resulting alkenyl fluorosulfonate was prone to decomposition during column chromatography and was therefore used in the next step without further purification. ${ }^{1} \mathrm{H} \mathrm{NMR}\left(\mathrm{CDCl}_{3}\right.$ $400 \mathrm{MHz}) \delta: 8.64(\mathrm{ddd}, J=4.7,1.8,0.9 \mathrm{~Hz}, 1 \mathrm{H}), 7.79(\mathrm{td}, J=7.9,1.8 \mathrm{~Hz}, 1$ H), $7.56(\mathrm{dt}, J=8.0,1.1 \mathrm{~Hz}, 1 \mathrm{H}), 7.33$ (ddd, $J=7.6,4.8,1.1 \mathrm{~Hz}, 1 \mathrm{H}), 6.40$ (dd, $J=3.4,0.7 \mathrm{~Hz}$, $1 \mathrm{H}), 5.63(\mathrm{dd}, J=3.5,1.6 \mathrm{~Hz}, 1 \mathrm{H}) \mathrm{ppm}$. Alkenyl sulfamate 22 was prepared following general procedure $\mathbf{C}$ (reaction time $=16 \mathrm{~h}$ ). Column chromatography $\left(\mathrm{SiO}_{2}\right.$, hexanes to 15:85 EtOAc:hexanes) afforded 22 as an off-white solid (183 mg, 55\%). Note: 22 rearranges to $\beta$ ketosulfonamide 47 over time during column chromatography and therefore should be purified rapidly. Less than $10 \%$ of 47 was observed when the purification time was below 20 min.

$\mathrm{R}_{\mathrm{f}}=0.50$ (3:7 EtOAc:hexanes)

${ }^{1} \mathrm{H}$ NMR $\left(\mathrm{CDCl}_{3}, 400 \mathrm{MHz}\right) \delta: 8.59-8.58$ (m, $\left.1 \mathrm{H}\right), 7.74-7.70(\mathrm{td}, J=7.8,1.8 \mathrm{~Hz}, 1 \mathrm{H}), 7.59-$ $7.57(\mathrm{~d}, J=8.0 \mathrm{~Hz}, 1 \mathrm{H}), 7.25-7.23(\mathrm{~m}, 1 \mathrm{H}), 6.19-6.18(\mathrm{~d}, J=2.1 \mathrm{~Hz}, 1 \mathrm{H}), 5.50(\mathrm{~d}, J=2.1 \mathrm{~Hz}$, $1 \mathrm{H}), 3.43-3.40$ (m, 4 H), 1.70-1.64 (m, 4 H), 1.60-1.54 (m, 2 H) ppm.

${ }^{13} \mathrm{C} \mathrm{NMR}\left(\mathrm{CDCl}_{3}, 126 \mathrm{MHz}\right) \delta: 151.7,151.4,149.5,136.8,123.7,119.5,104.4,48.1,25.1,23.4$ ppm.

HRMS(+ESI): calc'd for $\mathrm{C}_{12} \mathrm{H}_{17} \mathrm{~N}_{2} \mathrm{O}_{3} \mathrm{~S}[\mathrm{M}+\mathrm{H}]^{+}$269.0954, found 269.0950.

mp: $47-49^{\circ} \mathrm{C}$ 


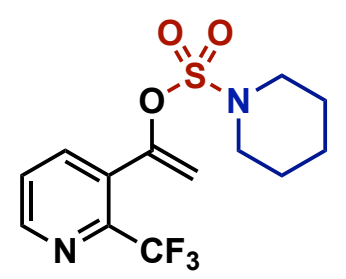

23

General procedure A was used with 1-[2-(trifluoromethyl)pyridin-3yl]ethan-1-one ( $40 \mathrm{mg}, 0.21 \mathrm{mmol}$ ) and anhydrous DCM at $0{ }^{\circ} \mathrm{C}$ for $1.5 \mathrm{~h}$. The resulting alkenyl fluorosulfonate was prone to decomposition during column chromatography and was therefore used in the next step without further purification. ${ }^{1} \mathrm{H} \mathrm{NMR}\left(\mathrm{CDCl}_{3}, 400 \mathrm{MHz}\right) \delta: 8.82(\mathrm{dd}, J=4.8,1.6$ $\mathrm{Hz}, 1 \mathrm{H}), 7.95$ (dd, $J=7.9,1.6 \mathrm{~Hz}, 1 \mathrm{H}), 7.60$ (td, $J=7.8,3.9 \mathrm{~Hz}, 1 \mathrm{H}), 5.78$ (dd, $J=4.1,1.2 \mathrm{~Hz}$, $1 \mathrm{H}), 5.48(\mathrm{~d}, J=4.1 \mathrm{~Hz}, 1 \mathrm{H}) \mathrm{ppm}$. Alkenyl sulfamate 23 was prepared following general procedure $\mathbf{C}$ (reaction time $=16 \mathrm{~h}$ ). Column chromatography $\left(\mathrm{SiO}_{2}\right.$, hexanes to 20:80 EtOAc:hexanes) afforded 23 as a colorless oil (49 mg, 72\%).

$\mathrm{R}_{\mathrm{f}}=0.38$ (1:4 EtOAc:hexanes)

${ }^{1} \mathrm{H}$ NMR $\left(\mathrm{CDCl}_{3}, 400 \mathrm{MHz}\right) \delta 8.74-8.72(\mathrm{~m}, 1 \mathrm{H}), 7.95-7.93(\mathrm{~m}, 1 \mathrm{H}), 7.54-7.52(\mathrm{dd}, J=7.9$, $4.7 \mathrm{~Hz}, 1 \mathrm{H}), 5.58$ (d, $J=3.0 \mathrm{~Hz}, 1 \mathrm{H}), 5.15-5.14$ (d, $J=3.0 \mathrm{~Hz}, 1 \mathrm{H}), 3.29-3.27$ (m, $4 \mathrm{H}), 1.65-$ $1.61(\mathrm{~m}, 4 \mathrm{H}), 1.56-1.52(\mathrm{~m}, 2 \mathrm{H}) \mathrm{ppm}$.

${ }^{13} \mathrm{C} \mathrm{NMR}\left(\mathrm{CDCl}_{3}, 126 \mathrm{MHz}\right) \delta: 149.7,149.1,145.1-145.1$ (q, $\left.J=33.8 \mathrm{~Hz}\right), 140.1,130.2,126.2$, 121.6-117.4 (q, $J=275.6 \mathrm{~Hz}), 107.5,47.8,24.9,23.4$ ppm.

${ }^{19} \mathrm{~F} \mathrm{NMR}\left(\mathrm{CDCl}_{3}, 470 \mathrm{MHz}\right) \delta:-64.1 \mathrm{ppm}$.

HRMS(+ESI): calc'd for $\mathrm{C}_{13} \mathrm{H}_{15} \mathrm{~F}_{2} \mathrm{~N}_{2} \mathrm{O}_{3} \mathrm{~S}[\mathrm{M}+\mathrm{H}]^{+} 337.0828$, found 337.0822 .

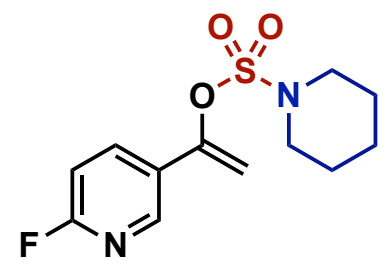

24

General procedure A was used with 1-(6-fluoropyridin-3-yl)ethanone $(57 \mathrm{mg}, 0.41 \mathrm{mmol})$ and anhydrous $\mathrm{DCM}$ at $0{ }^{\circ} \mathrm{C}$ for $1.5 \mathrm{~h}$. The resulting alkenyl fluorosulfonate was prone to decomposition during column chromatography and was therefore used in the next step without further purification. ${ }^{1} \mathrm{H} \mathrm{NMR}\left(\mathrm{CDCl}_{3}, 400 \mathrm{MHz}\right) \delta: 8.45(\mathrm{~d}, J=2.6 \mathrm{~Hz}, 1 \mathrm{H})$, $7.95(\mathrm{ddd}, J=8.6,7.2,2.7 \mathrm{~Hz}, 1 \mathrm{H}), 7.03(\mathrm{dd}, J=8.6,3.1 \mathrm{~Hz}, 1 \mathrm{H}), 5.69$ (d, $J=4.5 \mathrm{~Hz}, 1 \mathrm{H}), 5.58$ $(\mathrm{dd}, J=4.6,1.4 \mathrm{~Hz}, 1 \mathrm{H}) \mathrm{ppm}$. Alkenyl sulfamate 24 was prepared following general procedure $\mathbf{C}($ reaction time $=16 \mathrm{~h})$. Column chromatography $\left(\mathrm{SiO}_{2}, 10: 90\right.$ to 20:80 EtOAc:hexanes $)$ afforded 24 as a yellow oil (31 mg, 26\%). Note: 24 rearranges to $\beta$-ketosulfonamide 49 over time during column chromatography and therefore should be purified rapidly. Less than $10 \%$ of 49 was observed when the purification time was below $20 \mathrm{~min}$. 
$\mathrm{R}_{\mathrm{f}}=0.46(1: 4$ EtOAc:hexanes $)$

${ }^{1} \mathrm{H} \mathrm{NMR}\left(\mathrm{CDCl}_{3}, 400 \mathrm{MHz}\right) \delta: 8.41(\mathrm{~d}, J=2.4 \mathrm{~Hz}, 1 \mathrm{H}), 7.97-7.92$ (ddd, $J=8.6,7.4,2.6 \mathrm{~Hz}, 1$ H), 6.96-6.93 (dd, $J=8.7,2.9 \mathrm{~Hz}, 1 \mathrm{H}), 5.44-5.42$ (q, $J=3.3 \mathrm{~Hz}, 2 \mathrm{H}), 3.40-3.38$ (t, $J=5.5 \mathrm{~Hz}$, $4 \mathrm{H}), 1.71-1.64$ (m, $4 \mathrm{H}), 1.62-1.55$ (m, 2 H) ppm.

${ }^{13} \mathrm{C} \mathrm{NMR}\left(\mathrm{CDCl}_{3}, 126 \mathrm{MHz}\right) \delta: 165.0,162.6,149.5,145.2-145.0$ (d, $\left.J=15.6 \mathrm{~Hz}\right), 138.5-138.4$ $(\mathrm{d}, J=8.3 \mathrm{~Hz}), 128.5-128.4(\mathrm{~d}, J=4.9 \mathrm{~Hz}), 109.7,109.3,103.2(\mathrm{~d}, J=1.4 \mathrm{~Hz}), 48.0,25.0,23.4$ ppm.

${ }^{19} \mathrm{~F} \mathrm{NMR}\left(\mathrm{CDCl}_{3}, 470 \mathrm{MHz}\right) \delta:-67.3 \mathrm{ppm}$.

HRMS(+APCI): calc'd for $\mathrm{C}_{12} \mathrm{H}_{15} \mathrm{FN}_{2} \mathrm{O}_{3} \mathrm{~S}[\mathrm{M}+\mathrm{H}]^{+} 287.0860$, found 287.0854.

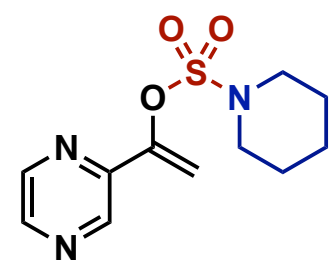

25

General procedure A was used with 2-acetylpyrazine (33 $\mathrm{mg}, 0.27 \mathrm{mmol}$ ) and anhydrous DCM at $0{ }^{\circ} \mathrm{C}$ for $1.5 \mathrm{~h}$. The resulting alkenyl fluorosulfonate was prone to decomposition during column chromatography and was therefore used in the next step without further purification. ${ }^{1} \mathrm{H} \mathrm{NMR}\left(\mathrm{CDCl}_{3}\right.$, $400 \mathrm{MHz}) \delta: 8.85(\mathrm{~d}, J=1.5 \mathrm{~Hz}, 1 \mathrm{H}), 8.64(\mathrm{~d}, J=2.5 \mathrm{~Hz}, 1 \mathrm{H}), 8.62(\mathrm{dd}, J$ $=2.4,1.5 \mathrm{~Hz}, 1 \mathrm{H}), 6.43(\mathrm{dd}, J=3.8,0.6 \mathrm{~Hz}, 1 \mathrm{H}), 5.76(\mathrm{dd}, J=3.8,1.5 \mathrm{~Hz}, 1 \mathrm{H}) \mathrm{ppm}$. Alkenyl sulfamate 25 was prepared following general procedure $\mathbf{C}$ (reaction time $=16 \mathrm{~h}$ ). Column chromatography $\left(\mathrm{SiO}_{2}\right.$, hexanes to 20:80 EtOAc:hexanes) and then preparatory TLC (30:70 EtOAc:hexanes) afforded 25 as a yellow oil (31 mg, 43\%). Note: 25 rearranges to $\beta$ ketosulfonamide 50 over time during column chromatography and therefore should be purified rapidly. Less than 10\% of $\mathbf{5 0}$ was observed when the purification time was below 20 min.

$\mathrm{R}_{\mathrm{f}}=0.55$ (1:2 EtOAc:hexanes)

${ }^{1} \mathrm{H}$ NMR $\left(\mathrm{CDCl}_{3}, 400 \mathrm{MHz}\right) \delta: 8.84(\mathrm{~s}, 1 \mathrm{H}), 8.56-8.54(\mathrm{q}, J=2.6 \mathrm{~Hz}, 1 \mathrm{H}), 6.24-6.23(\mathrm{~d}, J=$ $2.5 \mathrm{~Hz}, 1 \mathrm{H}), 5.63-5.63(\mathrm{~d}, J=2.5 \mathrm{~Hz}, 2 \mathrm{H}), 3.48-3.45(\mathrm{t}, J=5.5 \mathrm{~Hz}, 4 \mathrm{H}), 1.74-1.69(\mathrm{p}, J=5.7$ $\mathrm{Hz}, 4 \mathrm{H}), 1.66-1.58(\mathrm{~m}, 2 \mathrm{H}) \mathrm{ppm}$.

${ }^{13} \mathrm{C} \mathrm{NMR}\left(\mathrm{CDCl}_{3}, 126 \mathrm{MHz}\right) \delta: 149.7,147.2,144.8,144.2,141.0,106.6,48.2,25.1,23.5 \mathrm{ppm}$. HRMS(+ESI): calc'd for $\mathrm{C}_{11} \mathrm{H}_{15} \mathrm{~N}_{3} \mathrm{O}_{3} \mathrm{~S}[\mathrm{M}+\mathrm{H}]^{+} 270.0907$, found 270.0904 


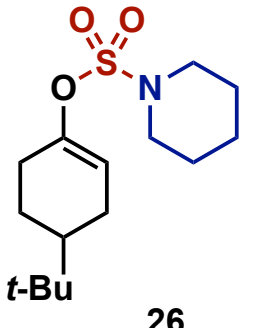

General procedure B was used with 4-tert-butylcyclohexanone (23 mg, 0.15 mmol). The resulting alkenyl fluorosulfonate was prone to decomposition during column chromatography and was therefore used in the next step without further purification. ${ }^{1} \mathrm{H} \mathrm{NMR}\left(\mathrm{CDCl}_{3}, 400 \mathrm{MHz}\right) \delta: 5.83(\mathrm{dd}, J=5.8,2.7 \mathrm{~Hz}$, $1 \mathrm{H}), 2.50-2.33$ (m, $2 \mathrm{H}), 2.20$ (s, $1 \mathrm{H}), 2.03-1.93$ (m, $2 \mathrm{H}), 1.50-1.31$ (m, 1

H), 1.29-1.23 (m, $2 \mathrm{H}), 0.92(\mathrm{~s}, 9 \mathrm{H}) \mathrm{ppm}$. Alkenyl sulfamate 26 was prepared following general procedure $\mathbf{C}$ (reaction time $=16 \mathrm{~h})$. Column chromatography $\left(\mathrm{SiO}_{2}, 2: 98\right.$ EtOAc:hexanes) afforded 26 as a yellow oil (34 $\mathrm{mg}, 75 \%)$.

$\mathrm{R}_{\mathrm{f}}=0.55$ (1:4 EtOAc:hexanes)

${ }^{1} \mathrm{H}$ NMR $\left(\mathrm{CDCl}_{3}, 400 \mathrm{MHz}\right) \delta: 5.64-5.61(\mathrm{dt}, J=5.0,2.2 \mathrm{~Hz}, 1 \mathrm{H}), 3.32-3.29(\mathrm{~m}, 4 \mathrm{H}), 2.35-$ 2.24 (m, 2 H), 2.17-2.10 (m, 1 H), 1.94-1.84 (m, 2 H), 1.70-1.64 (m, 4 H), 1.59-1.53 (m, 2 H), 1.34-1.25 (m, $2 \mathrm{H}), 0.88$ (s, $9 \mathrm{H}) \mathrm{ppm}$.

${ }^{13} \mathrm{C} \mathrm{NMR}\left(\mathrm{CDCl}_{3}, 126 \mathrm{MHz}\right) \delta: 148.4,115.3,47.9,43.4,32.2,28.6,27.4,25.3,25.1,24.2,23.7$ ppm.

HRMS(+ESI): ): calc'd for $\mathrm{C}_{15} \mathrm{H}_{27} \mathrm{NO}_{3} \mathrm{~S}\left[\mathrm{M}+\mathrm{H}^{+}\right]$302.1784, found 302.1778

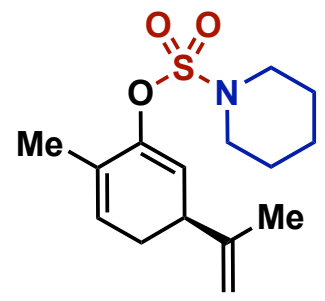

27

General procedure B was used with (+)-Carvone $(23 \mu \mathrm{L}, 0.15 \mathrm{mmol})$. The resulting alkenyl fluorosulfonate was prone to decomposition during column chromatography and was therefore used in the next step without further purification. ${ }^{1} \mathrm{H} \mathrm{NMR}\left(\mathrm{CDCl}_{3}, 400 \mathrm{MHz}\right) \delta: 5.71$ (ddd, $J=11.5,4.4,1.9 \mathrm{~Hz}$, $2 \mathrm{H}), 4.82(\mathrm{~d}, J=1.6 \mathrm{~Hz}, 2 \mathrm{H}), 3.18$ (ddd, $J=11.6,9.2,4.2 \mathrm{~Hz}, 1 \mathrm{H}), 2.43-$ $2.21(\mathrm{~m}, 2 \mathrm{H}), 1.83(\mathrm{q}, J=1.9 \mathrm{~Hz}, 3 \mathrm{H}), 1.76(\mathrm{t}, J=1.2 \mathrm{~Hz}, 3 \mathrm{H}) \mathrm{ppm}$. Alkenyl sulfamate 27 was prepared following general procedure $\mathbf{C}$ (reaction time $=16 \mathrm{~h})$. Column chromatography $\left(\mathrm{SiO}_{2}\right.$, 2:98 EtOAc:hexanes) afforded 27 as a yellow oil (30 mg, 67\%).

$\mathrm{R}_{\mathrm{f}}=0.62(1: 4$ EtOAc:hexanes)

${ }^{1} \mathrm{H}$ NMR $\left(\mathrm{CDCl}_{3}, 400 \mathrm{MHz}\right) \delta: 5.65-5.64(\mathrm{~d}, J=4.1 \mathrm{~Hz}, 1 \mathrm{H}), 5.63-5.60(\mathrm{td}, J=4.5,1.9 \mathrm{~Hz}, 1$ H), 4.81 (s, $1 \mathrm{H}), 4.81-4.76$ (q, $1 \mathrm{H}), 3.37-3.35$ (m, 4 H), 3.14-3.08 (ddd, $J=12.5,8.9,4.1 \mathrm{~Hz}, 1$ H), 2.33-2.15 (m, 2 H), 1.80-1.79 (d, $J=2.0$ Hz, 3 H), 1.75 (br. s., 3 H), 1.71-1.65 (m, 4 H), 1.611.54 (m, $2 \mathrm{H})$ ppm. 
${ }^{13} \mathrm{C} \mathrm{NMR}\left(\mathrm{CDCl}_{3}, 126 \mathrm{MHz}\right) \delta: 147.0,146.5,129.1,124.8,116.0,111.3,48.1,41.5,27.9,25.13$, 23.6, 20.8, $17.2 \mathrm{ppm}$.

HRMS(+ESI): calc'd for $\mathrm{C}_{15} \mathrm{H}_{23} \mathrm{NO}_{3} \mathrm{~S}\left[\mathrm{M}+\mathrm{H}^{+}\right]$298.1471, found 298.1466.

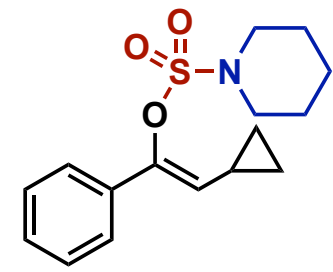

53

General procedure A was used with 2-cyclopropyl-1-phenylethanone (500 $\mathrm{mg}, 3.10 \mathrm{mmol}$ ) and anhydrous DCM at $0{ }^{\circ} \mathrm{C}$ for $1.5 \mathrm{~h}$. The resulting alkenyl fluorosulfonate was prone to decomposition during column chromatography and was therefore used in the next step without further purification. ${ }^{1} \mathrm{H}$ NMR $\left(\mathrm{CDCl}_{3}, 400 \mathrm{MHz}\right) \delta: 7.49-7.31(\mathrm{~m}, 5 \mathrm{H}), 5.31(\mathrm{~d}, J=10.0 \mathrm{~Hz}, 1 \mathrm{H}), 1.92-$ $1.84(\mathrm{~m}, 1 \mathrm{H}), 1.06-1.00(\mathrm{~m}, 2 \mathrm{H}), 0.64(\mathrm{dt}, J=6.6,4.5 \mathrm{~Hz}, 2 \mathrm{H}) \mathrm{ppm}$.

Alkenyl sulfamate $\mathbf{5 3}$ was prepared following general procedure $\mathbf{C}$ (reaction time $=48 \mathrm{~h}$ ). Column chromatography ( $\mathrm{SiO}_{2}$, hexanes to 1:99 $\mathrm{Et}_{2} \mathrm{O}$ :hexanes) afforded $\mathbf{5 3}$ as a white solid (72 $\mathrm{mg}, 15 \%)$. A small amount of E-isomer $(<3 \%)$ was detected by NMR.

$\mathrm{R}_{\mathrm{f}}=0.50$ (1:9 EtOAc:hexanes)

${ }^{1} \mathrm{H}$ NMR $\left(\mathrm{CDCl}_{2} 400 \mathrm{MHz}\right) \delta: 7.44-7.42(\mathrm{dd}, J=7.3,1.9 \mathrm{~Hz}, 2 \mathrm{H}), 7.34-7.31$ (t, $\left.J=7.5 \mathrm{~Hz}, 2 \mathrm{H}\right)$, 7.29-7.26 (m, $1 \mathrm{H}), 5.10-5.08$ (d, $J=10.0 \mathrm{~Hz}, 1 \mathrm{H}), 3.26-3.24$ (m, $4 \mathrm{H}), 2.08-2.01$ (dddd, $J=$ 12.8, 9.7, 8.1, 4.7 Hz, $1 \mathrm{H}), 1.61-1.56$ (m, 4 H), 1.52-1.47 (m, 2 H), 0.96-0.92 (m, 2 H), 0.55$0.52(\mathrm{dt}, J=6.6,4.5 \mathrm{~Hz}, 2 \mathrm{H})$.

${ }^{13} \mathrm{C} \mathrm{NMR}\left(\mathrm{CDCl}_{3}, 126 \mathrm{MHz}\right) \delta: 145.8,135.9,128.5,128.2,127.3,125.4,47.9,25.2,23.7,9.9,7.8$. ppm.

HRMS(+ESI): calc'd for C16H21NO3S [M+H] 308.1315 , found 308.1313.

mp: $59-60^{\circ} \mathrm{C}$

Representative Synthesis of Alkenyl Sulfamates (1 mmol scale)<smiles>O=S(=O)(F)OC1=CCCc2ccccc21</smiles>

$\mathrm{Ca}\left(\mathrm{NTf}_{2}\right)_{2}$ (1.1 equiv) $H N R_{1} R_{2}$ (1.05 equiv) DABCO (1.5 equiv)

THF, rt, $48 \mathrm{~h}$

2<smiles>C#CCNS(=O)(=O)OC1=CCCc2ccccc21</smiles>

12 
To a flame-dried $25 \mathrm{~mL}$ round-bottom-flask equipped with a stir bar, alkenyl fluorosulfonate 2 (228 mg, $1.0 \mathrm{mmol}), \mathrm{Ca}\left(\mathrm{NTf}_{2}\right)_{2}$ (660 mg, $1.1 \mathrm{mmol}, 1.10$ equiv) and DABCO (168 mg, $1.5 \mathrm{mmol}$, 1.50 equiv) were added and the flask was evacuated 3 times and placed under an atmosphere of nitrogen. Propargylamine ( $67 \mu \mathrm{L}, 1.05 \mathrm{mmol}, 1.05$ equiv) was added followed by dry THF (2.0 $\mathrm{mL}, \mathrm{C}=0.5 \mathrm{M}$ ). The solution was stirred at room temperature and monitored by TLC. Once full consumption of the starting material was reached after $48 \mathrm{~h}$, sat. $\mathrm{NH}_{4} \mathrm{Cl}(5 \mathrm{~mL})$ was added and the resulting mixture was extracted using EtOAc $(3 \times 15 \mathrm{~mL})$. The combined organic phases were washed with brine, dried over $\mathrm{MgSO}_{4}$, and the solvent was removed under reduced pressure. Column chromatography ( $\mathrm{SiO}_{2}, 2: 98$ to 5:95 EtOAc:hexanes) afforded $\mathbf{1 2}$ as an off-white solid (184 mg, 70\%).

\section{Chiral HPLC Analysis of Alkenyl Sulfamates $(S)-\mathbf{6}$ and $r a c-6$}

Chiral HPLC was run with compounds (S)-6 and rac-6 using an Agilent 1260 Infinity II HPLC and a Chiralcel(R) OD-H column that is $4.6 \mathrm{~mm}$ I.D. $\times 250 \mathrm{~mm}$ in length and particle size of 5 $\mu \mathrm{m}$. The compounds were eluted with hexane/isopropanol 70/30 at a flow rate of $1 \mathrm{~mL} / \mathrm{min}$. Detection was done by UV at 210, 254, and $280 \mathrm{~nm}$.

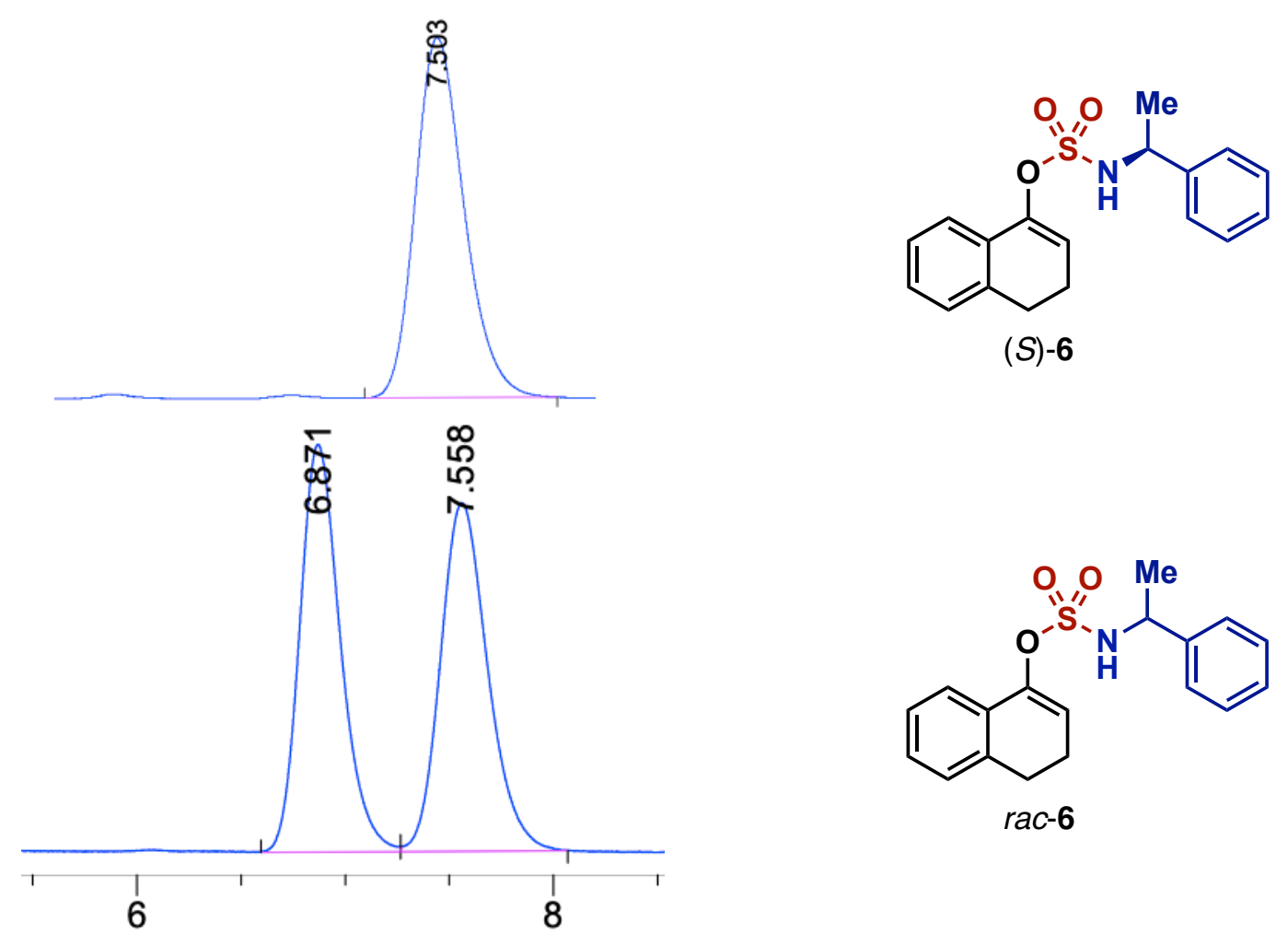

Figure S1. Top: HPLC trace for $(S)-6$; retention time $7.50 \mathrm{~min}$. Bottom: HPLC trace for rac-6; retention time $6.87 \mathrm{~min}$, area $276.96 \mathrm{mAU} . \mathrm{s}$ and retention time $7.56 \mathrm{~min}$, area $275.94 \mathrm{mAU} . \mathrm{s}$. 


\section{Chiral HPLC Analysis of Alkenyl Sulfamates $(S)-\mathbf{1 5}$ and $\mathrm{rac}-\mathbf{1 5}$}

Chiral HPLC was run with compounds (S)-15 and rac-15 using an Agilent 1260 Infinity II HPLC and a Chiralcel(R) OD-H column that is $4.6 \mathrm{~mm}$ I.D. x $250 \mathrm{~mm}$ in length and particle size of 5 $\mu \mathrm{m}$. The compounds were eluted with hexane/isopropanol $70 / 30$ at a flow rate of $1 \mathrm{~mL} / \mathrm{min}$. Detection was done by UV at 210, 254, and $280 \mathrm{~nm}$.

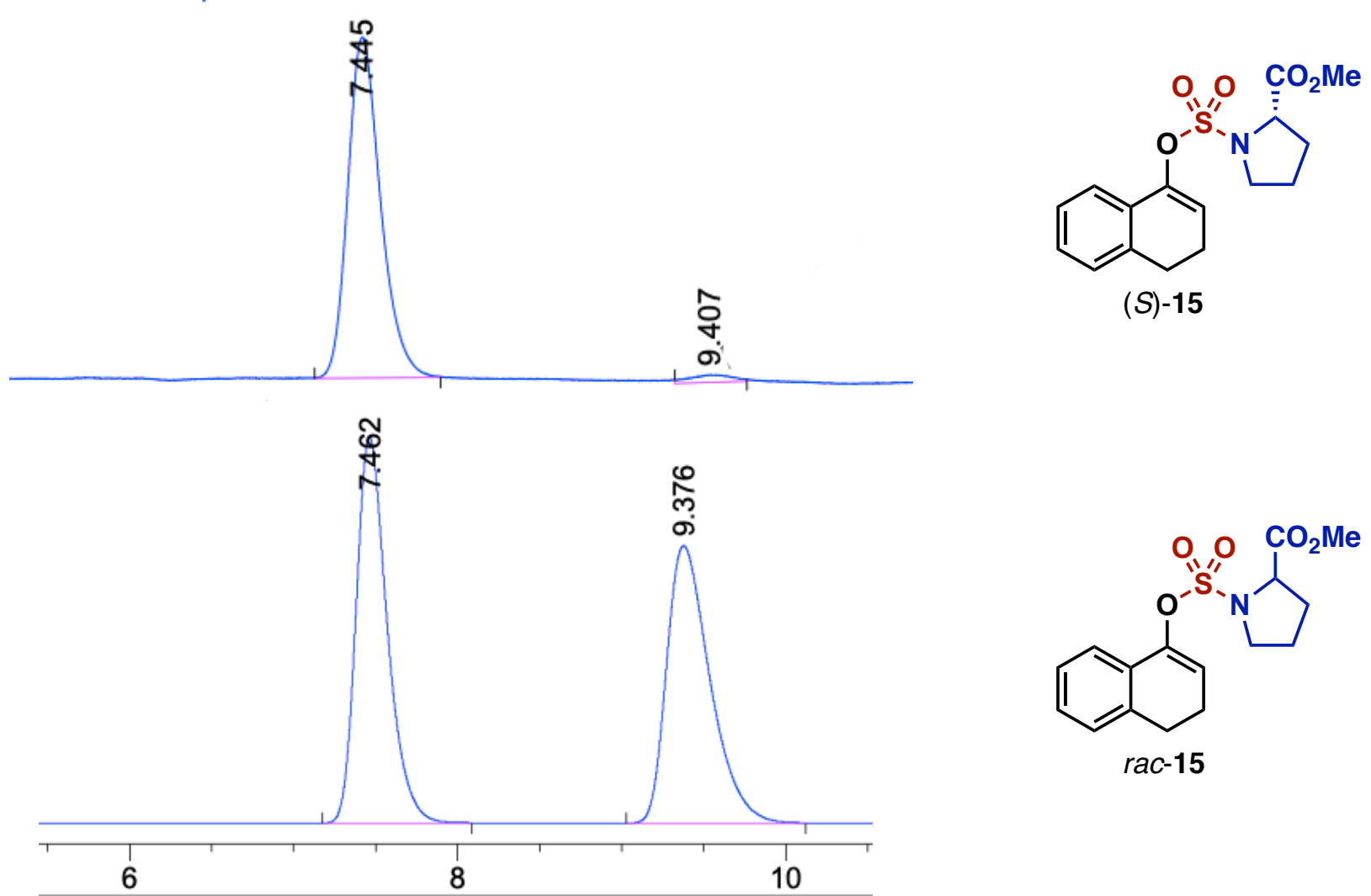

Figure S2. Top: HPLC trace for $(S)-15$; retention time $7.45 \mathrm{~min}$, area $197.58 \mathrm{mAU} . \mathrm{s}$ (major product), retention time $9.41 \mathrm{~min}$, area 5.63 mAU.s (minor product). Bottom: HPLC trace for rac-15; retention time $7.46 \mathrm{~min}$, area 1391.1 mAU.s and retention time $9.38 \mathrm{~min}$, area $1391.5 \mathrm{mAU} . \mathrm{s}$.

This analysis revealed that less than 3\% isomerization took place during the coupling of L- or Dproline methyl ester with $\mathbf{2}$. 


\section{Attempts to Induce the Rearrangements with a Lewis or Brønsted Acid}

To a flame-dried $4 \mathrm{~mL}$ dram vial equipped with a stir bar, alkenyl sulfamate 4 (10 mg, $0.032 \mathrm{mmol}$, 1.00 equiv) was added and placed under nitrogen atmosphere after evacuating the flask 3 times. Then, dry THF $(0.16 \mathrm{~mL}, \mathrm{C}=0.2 \mathrm{M})$ and the Lewis or Brønsted acid (0.035 mmol, 1.12 equiv) were added in that order. After stirring the mixture for $12 \mathrm{~h}$, the solvent was removed in vacuo, and the crude material was analyzed by ${ }^{1} \mathrm{H} \mathrm{NMR}$

Table S3. Attempts of 1,3-rearrangement using Lewis or Brønsted acid

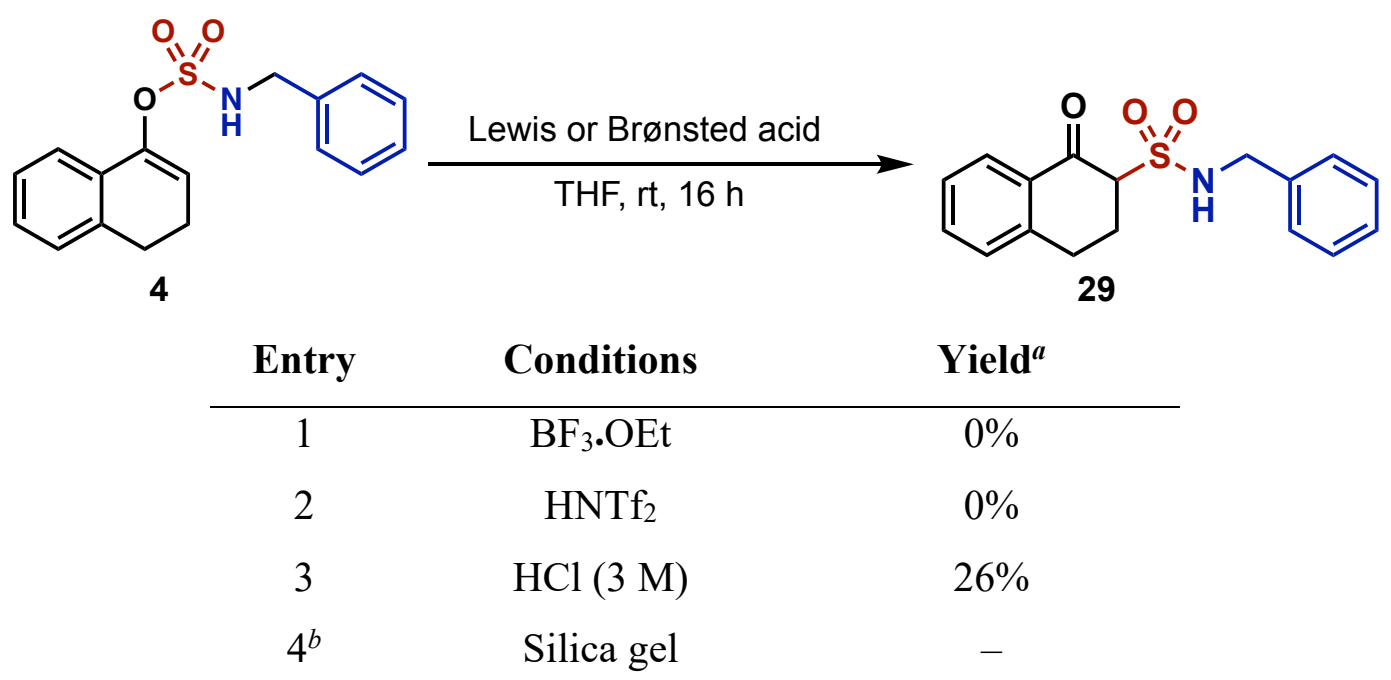

All reactions were carried out using 1.1 equiv of the acid under a nitrogen atmosphere. ${ }^{a}$ Yields were determined using 1,4-dimethoxybenzene as internal standard. ${ }^{b}$ Reaction was stirred at $60{ }^{\circ} \mathrm{C}$ for 12 hours.

Optimization of the Photochemical 1,3-Rearrangement with Substrates $\mathbf{4}$ and $\mathbf{3}$

Alkenyl sulfamate 4 or 3 ( $0.036 \mathrm{mmol}, 1.0$ equiv), PC ( $0.0036 \mathrm{mmol}, 0.01$ equiv) were placed into a $4 \mathrm{~mL}$ vial (borosilicate glass) and DMSO $(0.38 \mathrm{~mL})$ and distilled water $(0.38 \mathrm{~mL})$ were added to the vial. The solution was irradiated with a $456 \mathrm{~nm}$ Kessil lamps (approximately $1 \mathrm{~cm}$ away from the light source) and stirred at room temperature for the indicated amount of time. Then water (5 $\mathrm{mL})$ was added and the resulting mixture was extracted using EtOAc $(3 \times 5 \mathrm{~mL})$. The combined organic phases were washed with brine and dried over $\mathrm{MgSO}_{4}$. The solvent was evaporated in vacuo and the crude material was analyzed by ${ }^{1} \mathrm{H}$ NMR. 
Table S4. Representative Conditions Screened for the Synthesis of $\beta$-Ketosulfonamide 29 from 4

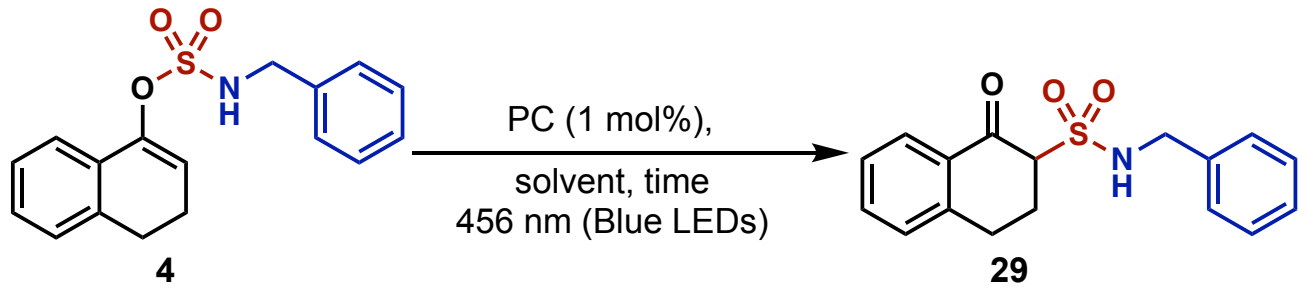

\begin{tabular}{|c|c|c|c|c|}
\hline Entry & Photocatalyst & Solvent & Time & Yield $^{\mathrm{a}}$ \\
\hline 1 & $\left(\operatorname{Ir}\left[\mathrm{dF}\left(\mathrm{CF}_{3}\right) \mathrm{ppy}_{2}\right] \mathrm{dtbpy}\right) \mathrm{PF}_{6}$ & $\mathrm{MeCN}$ & $16 \mathrm{~h}$ & $43 \%$ \\
\hline 2 & $\left(\operatorname{Ir}\left[\mathrm{dF}\left(\mathrm{CF}_{3}\right) \mathrm{ppy}_{2}\right] \mathrm{dtbpy}\right) \mathrm{PF}_{6}$ & THF & $16 \mathrm{~h}$ & $67 \%$ \\
\hline 3 & $\left(\operatorname{Ir}\left[\mathrm{dF}\left(\mathrm{CF}_{3}\right) \mathrm{ppy}_{2}\right] \mathrm{dtbpy}\right) \mathrm{PF}_{6}$ & DCM & $16 \mathrm{~h}$ & $68 \%$ \\
\hline 4 & $\left(\operatorname{Ir}\left[\mathrm{dF}\left(\mathrm{CF}_{3}\right)\right.\right.$ ppy 2$\left.] \mathrm{dtbpy}\right) \mathrm{PF}_{6}$ & DMSO & $16 \mathrm{~h}$ & $88 \%$ \\
\hline 5 & $\left(\operatorname{Ir}\left[\mathrm{dF}\left(\mathrm{CF}_{3}\right) \mathrm{ppy}_{2}\right] \mathrm{dtbpy}\right) \mathrm{PF}_{6}$ & DMSO: $\mathrm{H}_{2} \mathrm{O}(1: 1)$ & $16 \mathrm{~h}$ & $95 \%$ \\
\hline 6 & $\operatorname{Ir}(\text { ppy })_{3}$ & DMSO: $\mathrm{H}_{2} \mathrm{O}(1: 1)$ & $16 \mathrm{~h}$ & $84 \%$ \\
\hline 7 & $\operatorname{eosin} B$ & DMSO: $\mathrm{H}_{2} \mathrm{O}(1: 1)$ & $16 \mathrm{~h}$ & $98 \%$ \\
\hline 8 & No PC & DMSO:H $\mathrm{H}_{2} \mathrm{O}(1: 1)$ & $16 \mathrm{~h}$ & $99 \%$ \\
\hline
\end{tabular}

All reactions were performed using $0.030 \mathrm{mmol}$ of substrate 4 . All reactions were degassed through "freezepump-thaw" technique and left under nitrogen atmosphere before irradiation with $456 \mathrm{~nm}$ LEDs. ${ }^{a}$ Yields were determined using 1,4-dimethoxybenzene as internal standard.

Table S5. Representative Conditions Screened for the Synthesis of $\beta$-Ketosulfonamide $\mathbf{2 8}$ from 3<smiles>O=C1c2cc(C3CCCC3)ccc2[C+]CC1S(=O)(=O)N1CCCC1</smiles>

\begin{tabular}{ccccc} 
Entry & PC (1mol\%) & Solvent & Time & Yield $^{c}$ \\
\hline $1^{a}$ & No PC & DMSO: $\mathrm{H}_{2} \mathrm{O}(1: 1)$ & $16 \mathrm{~h}$ & $0 \%$ \\
$2^{a}$ & eosin B & DMSO: $\mathrm{H}_{2} \mathrm{O}(1: 1)$ & $16 \mathrm{~h}$ & $0 \%$ \\
$3^{a}$ & $9-$ fluorenone & DMSO: $\mathrm{H}_{2} \mathrm{O}(1: 1)$ & $16 \mathrm{~h}$ & $50 \%$ \\
$4^{a}$ & $\mathrm{Ir}(\mathrm{ppy})_{3}$ & DMSO: $\mathrm{H}_{2} \mathrm{O}(1: 1)$ & $16 \mathrm{~h}$ & $70 \%$ \\
$5^{a}$ & $\mathrm{Ir}(\mathrm{ppy})_{3}$ & DMSO: $\mathrm{H}_{2} \mathrm{O}(1: 1)$ & $48 \mathrm{~h}$ & $95 \%$ \\
$\mathbf{6}^{\boldsymbol{b}}$ & $\mathbf{I r}(\mathbf{p p y})_{3}$ & DMSO: $\mathrm{H}_{\mathbf{2}} \mathbf{O}(\mathbf{1 : 1})$ & $\mathbf{6} \mathbf{~ h}$ & $\mathbf{9 7 \%}$
\end{tabular}

All reactions were performed using $0.036 \mathrm{mmol}$ of substrate $3 .{ }^{a}$ The reactions were degassed through "freeze-pump-thaw" technique before irradiation with $456 \mathrm{~nm}$ LEDs. ${ }^{b}$ The reaction was performed without degassing. ${ }^{c}$ Yields were determined using 1,4-dimethoxybenzene as internal standard. 
Probing the Effect of Oxygen in the Photochemical 1,3-rearrangement<smiles>O=S(=O)(OC1=CCCc2ccccc21)N1CCCC1</smiles>

3

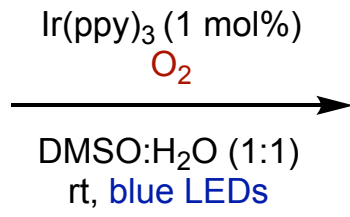

rt, blue LEDs<smiles>O=C1c2ccccc2CCC1S(=O)(=O)N1CCCC1</smiles>

28

Alkenyl sulfamate 3 (0.15 mmol, 1.0 equiv), $\operatorname{Ir}(\text { ppy })_{3}(1.0 \mathrm{mg}, 0.0015 \mathrm{mmol}, 0.01$ equiv) were placed into a $4 \mathrm{~mL}$ vial (borosilicate glass) and DMSO $(0.38 \mathrm{~mL})$ and distilled water $(0.38 \mathrm{~mL})$ were added to the vial. The solution was saturated with an oxygen balloon for 10 min while sonicating. The cloudy solution was irradiated with a $456 \mathrm{~nm}$ Kessil lamps (approximately $1 \mathrm{~cm}$ away from the light source) and stirred at room temperature for $16 \mathrm{~h}$. Then water $(5 \mathrm{~mL})$ was added and the resulting mixture was extracted using EtOAc $(3 \times 5 \mathrm{~mL})$. The combined organic phases were washed with brine and dried over $\mathrm{MgSO}_{4}$. The solvent was evaporated in vacuo and column chromatography ( $\mathrm{SiO}_{2}, 10: 90$ to 15:85 EtOAc:hexanes) afforded 28 as a pale-yellow oil (89\%).
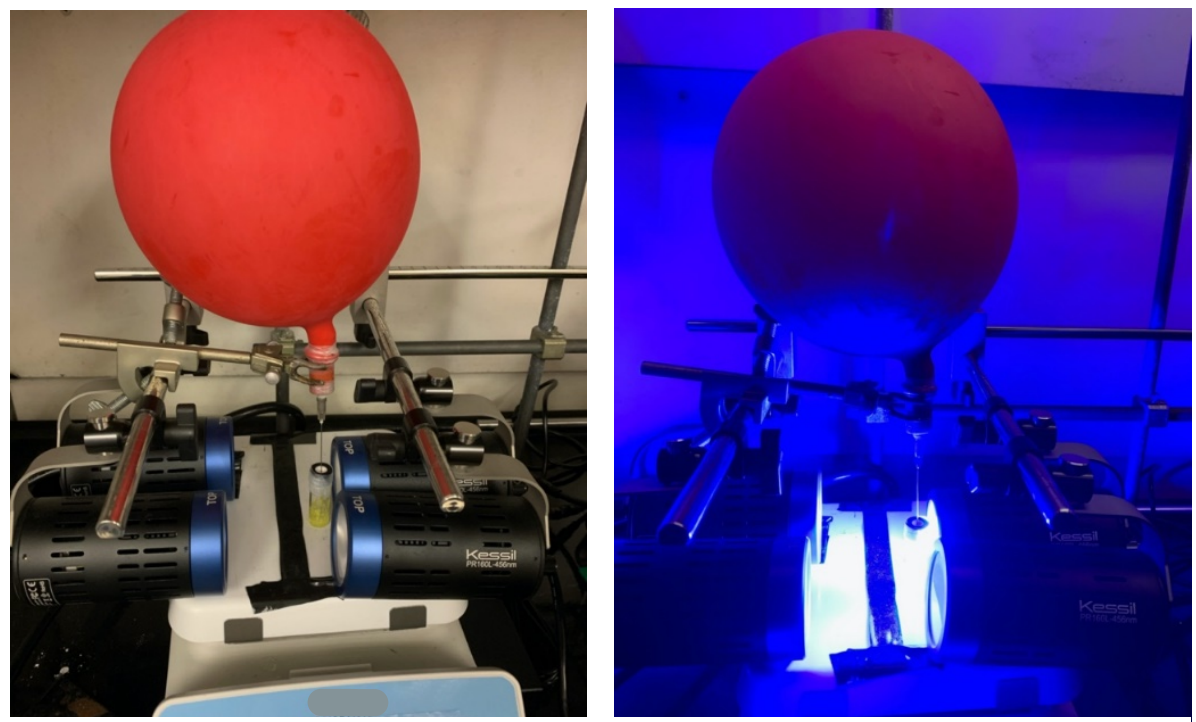

Figure S3. Left: Mixture of $\operatorname{Ir}(\mathrm{ppy})_{3}$, alkenyl sulfamate 28, and DMSO: $\mathrm{H}_{2} \mathrm{O}$ mixture saturated with oxygen. Right: followed by irradiation with blue LEDs $(456 \mathrm{~nm})$. 


\section{$\underline{\text { Synthesis of } \beta \text {-Ketosulfonamides: General Procedure D }}$}

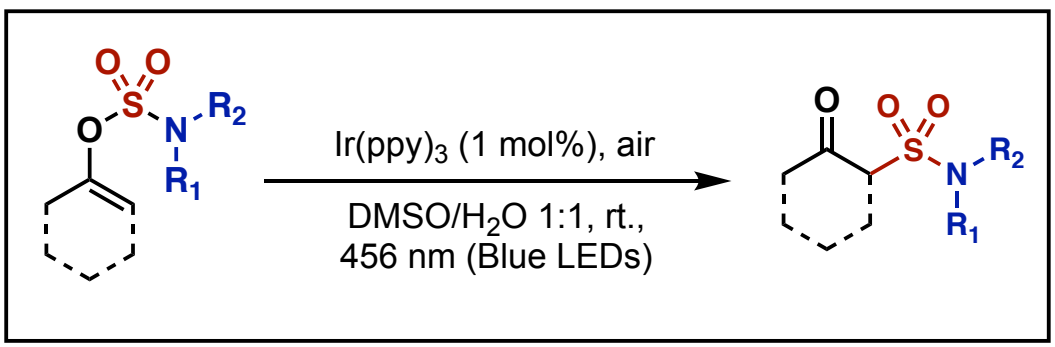

Alkenyl sulfamate ( $0.15 \mathrm{mmol}, 1.0$ equiv) and $\operatorname{Ir}(\mathrm{ppy})_{3}(1 \mathrm{mg}, 0.0015 \mathrm{mmol}, 0.01$ equiv) were placed into a $4 \mathrm{~mL}$ vial (borosilicate glass). DMSO $(0.38 \mathrm{~mL})$ and distilled water $(0.38 \mathrm{~mL})$ were added to the vial. The cloudy solution was irradiated by $456 \mathrm{~nm}$ Kessil lamps (approximately 1 $\mathrm{cm}$ away from the light source) and stirred at room temperature until the full consumption of starting material was observed by TLC. The mixture then was diluted with EtOAc $(3 \mathrm{~mL})$, water $(3 \mathrm{~mL})$, and extracted with EtOAc $(3 \times 3 \mathrm{~mL})$ and the combined organic layers were washed with brine (3 mL) before drying over $\mathrm{MgSO}_{4}$. The solvent was evaporated in vacuo and the crude material was purified via column chromatography.
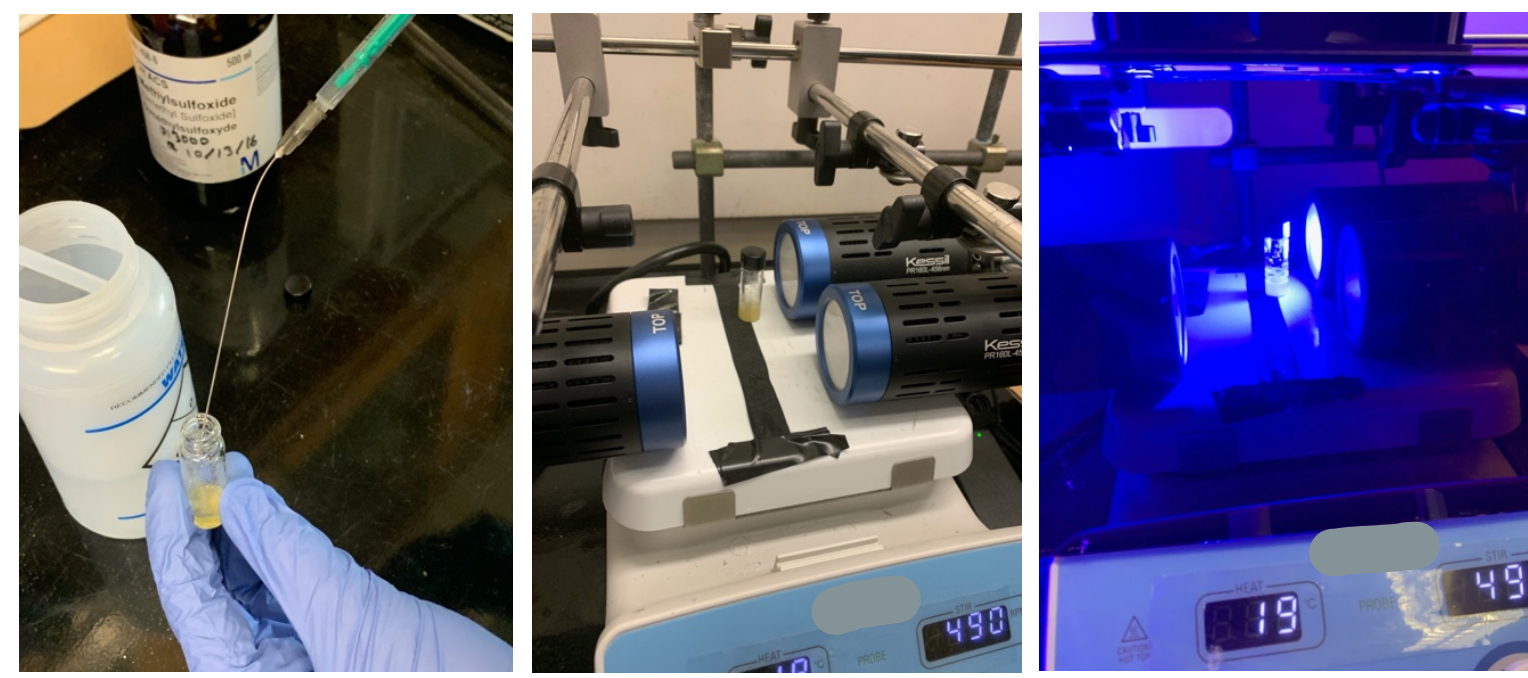

Figure S4. Left: Yellow suspension observed after addition of DMSO and water to the alkenyl sulfamate and $\operatorname{Ir}(\mathrm{ppy})_{3}$. Middle and right: The reaction was placed $1 \mathrm{~cm}$ away from the blue light source and irradiated. 


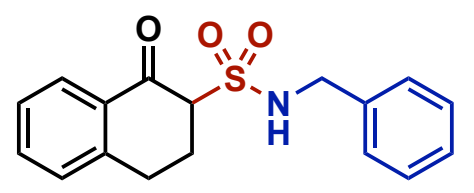

29

$\beta$-Ketosulfonamide 29 was prepared from alkenyl sulfamate 4 (47 $\mathrm{mg}, 0.15 \mathrm{mmol}$ ) following general procedure $\mathbf{D}$ (reaction time $=8$ h). Column chromatography $\left(\mathrm{SiO}_{2}, 10: 90\right.$ to 15:85 EtOAc:hexanes) afforded 29 as a white solid (47 $\mathrm{mg}, 99 \%$ ).

$\mathrm{R}_{\mathrm{f}}=0.22(1: 4$ EtOAc:hexanes)

${ }^{1} \mathrm{H}$ NMR $\left(\mathrm{CDCl}_{3}, 400 \mathrm{MHz}\right) \delta: 8.05-8.03$ (m, $\left.1 \mathrm{H}\right), 7.56-7.52(\mathrm{td}, J=7.5,1.4 \mathrm{~Hz}, 1 \mathrm{H}), 7.41-$ $7.28(\mathrm{~m}, 7 \mathrm{H}), 5.41-5.38(\mathrm{t}, J=6.4 \mathrm{~Hz}, 1 \mathrm{H}), 4.47-4.31(\mathrm{~m}, 2 \mathrm{H}), 3.90-3.86(\mathrm{dd}, J=9.1,5.4 \mathrm{~Hz}$, $1 \mathrm{H}), 3.31-3.24$ (dt, $J=16.9,5.6 \mathrm{~Hz}, 1 \mathrm{H}), 3.01-2.93$ (ddd, $J=16.8,9.1,4.9 \mathrm{~Hz}, 1 \mathrm{H}), 2.74-2.59$ (ddtd, $J=22.9,13.8,8.7,5.4 \mathrm{~Hz}, 2 \mathrm{H}$ ) ppm.

${ }^{13} \mathrm{C} \mathrm{NMR}\left(\mathrm{CDCl}_{3}, 126 \mathrm{MHz}\right) \delta: 190.8,144.0,136.6,134.9,131.7,129.1,129 ., 128.3,128.2,128.2$, $127.4,65.8,47.9,27.3,24.4$ ppm.

HRMS(+APCl) calc'd for $\mathrm{C}_{17} \mathrm{H}_{17} \mathrm{NO}_{3} \mathrm{~S}\left[\mathrm{M}+\mathrm{H}^{+}\right]$316.1002, found 316.0996.

m.p: $87-89^{\circ} \mathrm{C}$

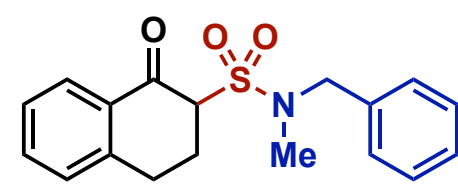

30

$\beta$-Ketosulfonamide 30 was prepared from alkenyl sulfamate 5 (49 $\mathrm{mg}, 0.15 \mathrm{mmol}$ ) following general procedure $\mathbf{D}$ (reaction time $=6$ h). Column chromatography ( $\mathrm{SiO}_{2}, 10: 90$ EtOAc:hexanes) afforded 30 as a colorless oil (47 $\mathrm{mg}, 95 \%)$.

$\mathrm{R}_{\mathrm{f}}=0.37$ (1:4 EtOAc:hexanes)

${ }^{1} \mathrm{H}$ NMR $\left(\mathrm{CDCl}_{3}, 400 \mathrm{MHz}\right) \delta: 8.09-8.06(\mathrm{dd}, J=7.9,1.5 \mathrm{~Hz}, 1 \mathrm{H}), 7.56-7.52(\mathrm{td}, J=7.5,1.5$ Hz, 1 H), 7.38-7.27 (m, 7 H), 4.50-4.26 (m, 2 H), 4.15-4.13 (t, J=4.8 Hz, 1 H), 3.63-3.54 (ddd, $J=16.4,11.3,4.7 \mathrm{~Hz}, 1 \mathrm{H}), 2.97-2.83$ (m, $2 \mathrm{H}), 2.84$ (s, $3 \mathrm{H}), 2.66-2.57$ (ddt, $J=14.4,11.5,5.2$ $\mathrm{Hz}, 1 \mathrm{H}) \mathrm{ppm}$.

${ }^{13} \mathrm{C} \mathrm{NMR}\left(\mathrm{CDCl}_{3}, 126 \mathrm{MHz}\right) \delta: 190.4,144.3,136.3,134.7,131.8,129.2,128.8,128.4,127.99$ (d, $J=8.0 \mathrm{~Hz}), 127.1,65.6,54.9,34.9,26.3,25.5 \mathrm{ppm}$.

HRMS(+ESI) calc'd for $\mathrm{C}_{18} \mathrm{H}_{19} \mathrm{NO}_{3} \mathrm{~S}\left[\mathrm{M}+\mathrm{H}^{+}\right] 330.1158$, found 330.1151 . 


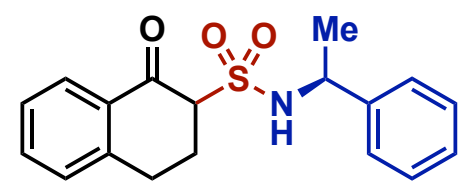

31

$\beta$-Ketosulfonamide $\mathbf{3 1}$ was prepared from alkenyl sulfamate $\mathbf{6}$ (49 $\mathrm{mg}, 0.15 \mathrm{mmol}$ ) following general procedure $\mathrm{D}$ (reaction time $=6$ h). Column chromatography ( $\mathrm{SiO}_{2}, 10: 90$ to 15:85 EtOAc:hexanes) afforded 31 as a colorless oil (47 mg, 95\%).

$\mathrm{R}_{\mathrm{f}}=0.37$ (1:4 EtOAc:hexanes)

${ }^{1} \mathrm{H}$ NMR $\left(\mathrm{CDCl}_{3}, 400 \mathrm{MHz}\right)$ mixture of diastereomers (dr. 1:1.4) (major) $\delta: ~ 7.99-7.96(\mathrm{dd}, J=$ 7.9, $1.5 \mathrm{~Hz}, 1 \mathrm{H}), 7.51-7.47$ (td, $J=7.4,1.5 \mathrm{~Hz}, 1 \mathrm{H}), 7.40-7.18(\mathrm{~m}, 7 \mathrm{H}), 5.64-5.62$ (d, $J=8.9$ Hz, 1 H), 4.78-4.70 (m, 1 H), 3.10-2.94 (m, 2 H), 2.78-2.63 (m, 1 H), 2.56-2.37 (m, 2 H), 1.62$1.60(\mathrm{~d}, J=7.0 \mathrm{~Hz}, 6 \mathrm{H})$; (minor) $\delta: 8.08-8.05(\mathrm{dd}, J=7.9,1.5 \mathrm{~Hz}, 1 \mathrm{H}), 7.56-7.52(\mathrm{td}, J=7.5$, $1.5 \mathrm{~Hz}, 1 \mathrm{H}), 7.40-7.18(\mathrm{~m}, 7 \mathrm{H}), 5.33-5.31(\mathrm{~d}, J=6.9 \mathrm{~Hz}, 1 \mathrm{H}), 4.78-4.70(\mathrm{~m}, 1 \mathrm{H}), 4.04-4.01$ $(\mathrm{dd}, J=9.8,5.0 \mathrm{~Hz}, 1 \mathrm{H}), 3.27-3.20$ (dt, $J=16.8,5.3 \mathrm{~Hz}, 1 \mathrm{H}), 3.10-2.94$ (m, $1 \mathrm{H}), 2.78-2.63$ (m, $1 \mathrm{H}), 2.56-2.37(\mathrm{~m}, 1 \mathrm{H}), 1.62-1.60(\mathrm{~d}, J=7.0 \mathrm{~Hz}, 6 \mathrm{H}) \mathrm{ppm}$.

${ }^{13} \mathrm{C} \mathrm{NMR}\left(\mathrm{CDCl}_{3}, 126 \mathrm{MHz}\right) \delta: 191.0,190.8,144.0,143.8,143.1,142.1,134.8,134.7,131.8$, $131.5,129.0,128.9,128.9,128.8,128.2,128.2,128.0,127.7,127.3,127.2,126.6,126.3,67.0$, 66.5, 54.9, 54.0, 27.6, 27.4, 24.7, 24.3, 24.0, 23.6. ppm.

HRMS(+APCl): calc'd for $\mathrm{C}_{18} \mathrm{H}_{19} \mathrm{NO}_{3} \mathrm{~S}\left[\mathrm{M}+\mathrm{H}^{+}\right]$330.1158, found 330.1151.

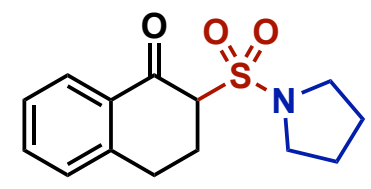

28

$\beta$-Ketosulfonamide 28 was prepared from alkenyl sulfamate 3 (42 $\mathrm{mg}$, $0.15 \mathrm{mmol}$ ) following general procedure $\mathrm{D}$ (reaction time $=6 \mathrm{~h}$ ). Column chromatography ( $\mathrm{SiO}_{2}, 10: 90$ to 15:85 EtOAc:hexanes) afforded as a paleyellow oil (41 mg, 97\%).

$\mathrm{R}_{\mathrm{f}}=0.40$ (1:4 EtOAc:hexanes)

${ }^{1} \mathrm{H}$ NMR $\left(\mathrm{CDCl}_{3}, 400 \mathrm{MHz}\right) \delta: 8.05-8.03(\mathrm{dd}, J=7.9,1.4 \mathrm{~Hz}, 1 \mathrm{H}), 7.54-7.50(\mathrm{td}, J=7.5,1.5$ $\mathrm{Hz}, 1 \mathrm{H}), 7.34-7.31$ (t, $J=7.6 \mathrm{~Hz}, 1 \mathrm{H}), 7.28-7.26$ (d, $J=9.3 \mathrm{~Hz}, 1 \mathrm{H}), 4.14-4.11$ (dd, $J=5.5,4.0$ Hz, $1 \mathrm{H}), 3.60-3.52$ (ddd, $J=16.6,11.5,4.7 \mathrm{~Hz}, 1 \mathrm{H}), 3.47-3.35$ (m, $4 \mathrm{H}), 2.94-2.88$ (dt, $J=17.0$, 4.6 Hz, 1 H), 2.84-2.78 (dq, $J=14.4,4.3 \mathrm{~Hz}, 1 \mathrm{H}), 2.60-2.51$ (ddt, $J=14.4,11.5,5.2 \mathrm{~Hz}, 1 \mathrm{H}$ ), 2.04-1.94 (m, 4 H) ppm.

${ }^{13} \mathrm{C} \mathrm{NMR}\left(\mathrm{CDCl}_{3}, 126 \mathrm{MHz}\right) \delta: 190.6,144.3,134.5,131.9,129.2,127.9,127.0,64.7,48.6,26.2$, 26.0, 25.5 ppm. 
HRMS(+APCl): calc'd for $\mathrm{C}_{14} \mathrm{H}_{17} \mathrm{NO}_{3} \mathrm{~S}\left[\mathrm{M}+\mathrm{H}^{+}\right]$280.1002, found 280.0.995.

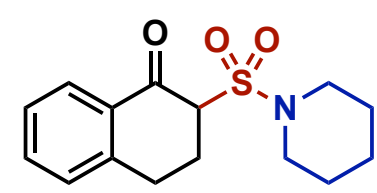

32

$\beta$-Ketosulfonamide 32 was prepared from alkenyl sulfamate 7 (44 mg, $0.15 \mathrm{mmol}$ ) following general procedure D (reaction time $=6 \mathrm{~h}$ ). Column chromatography $\left(\mathrm{SiO}_{2}, 10: 90\right.$ to 15:85 EtOAc:hexanes) afforded 32 as a colorless oil (42 mg, 95\%).

$\mathrm{R}_{\mathrm{f}}=0.43$ (1:4 EtOAc:hexanes)

${ }^{1} \mathrm{H} \mathrm{NMR}\left(\mathrm{CDCl}_{3}, 400 \mathrm{MHz}\right) \delta: 8.04-8.02(\mathrm{dd}, J=8.0,1.4 \mathrm{~Hz}, 1 \mathrm{H}), 7.54-7.49$ (td, $J=7.5,1.5$ $\mathrm{Hz}, 1 \mathrm{H}), 7.34-7.26(\mathrm{t}, J=7.6 \mathrm{~Hz}, 1 \mathrm{H}), 7.28-7.26\left(\mathrm{~d}, J=6.7 \mathrm{~Hz}, 1 \mathrm{H}\right.$, overlaps with $\left.C D C l_{3}\right)$, 4.02-4.00 (t, $J=4.7 \mathrm{~Hz}, 1 \mathrm{H}), 3.58-3.50$ (ddd, $J=16.7,11.5,4.7 \mathrm{~Hz}, 1 \mathrm{H}), 3.40-3.29$ (m, $4 \mathrm{H})$, 2.94-2.87 (dt, $J=17.1,4.6 \mathrm{~Hz}, 1 \mathrm{H}), 2.83-2.76$ (dq, $J=14.4,4.3 \mathrm{~Hz}, 1 \mathrm{H}), 2.58-2.49$ (ddt, $J=$ 14.4, 11.6, $5.2 \mathrm{~Hz}, 1 \mathrm{H}), 1.68-1.57$ (m, $6 \mathrm{H}) \mathrm{ppm}$.

${ }^{13} \mathrm{C} \mathrm{NMR}\left(\mathrm{CDCl}_{3}, 126 \mathrm{MHz}\right) \delta: 190.4,144.2,134.5,131.9,129.2,128.0,127.0,65.7,47.4,26.3$, 26.1, 25.7, $24.0 \mathrm{ppm}$.

HRMS(+APCl): calc'd for $\mathrm{C}_{15} \mathrm{H}_{19} \mathrm{NO}_{3} \mathrm{~S}\left[\mathrm{M}+\mathrm{H}^{+}\right]$294.1158, found 294.1150.

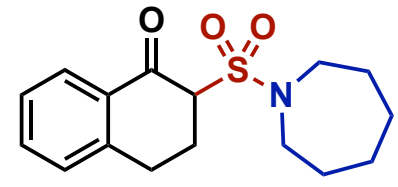

33

$\beta$-Ketosulfonamide 33 was prepared from alkenyl sulfamate 8 (46 mg, $0.15 \mathrm{mmol}$ ) following general procedure $\mathbf{D}$ (reaction time $=6 \mathrm{~h}$ ). Column chromatography $\left(\mathrm{SiO}_{2}, 10: 90\right.$ to 15:85 EtOAc:hexanes) afforded $\mathbf{3 3}$ as a colorless oil (36 mg, 79\%).

$\mathrm{R}_{\mathrm{f}}=0.56(1: 4$ EtOAc:hexanes $)$

${ }^{1} \mathrm{H}$ NMR $\left(\mathrm{CDCl}_{3}, 400 \mathrm{MHz}\right) \delta: 8.04-8.01(\mathrm{dd}, J=8.0,1.4 \mathrm{~Hz}, 1 \mathrm{H}), 7.53-7.49$ (td, $J=7.5,1.5$ $\mathrm{Hz}, 1 \mathrm{H}), 7.34-7.30(\mathrm{t}, J=7.6 \mathrm{~Hz}, 1 \mathrm{H}), 7.28-7.26$ (d, $J=7.2 \mathrm{~Hz}, 1 \mathrm{H}), 4.06-4.04$ (dd, $J=5.5,3.7$ Hz, $1 \mathrm{H}), 3.62-3.54$ (ddd, $J=16.7,11.8,4.8 \mathrm{~Hz}, 1 \mathrm{H}), 3.42-3.31$ (m, $4 \mathrm{H}), 2.92-2.77$ (m, $2 \mathrm{H})$, 2.58-2.48 (ddt, $J=14.5,11.1,5.2 \mathrm{~Hz}, 1 \mathrm{H}), 1.81-1.58$ (m, $8 \mathrm{H})$ ppm.

$\mathrm{NMR}\left(\mathrm{CDCl}_{3}, 126 \mathrm{MHz}\right) \delta: 190.4,144.3,134.5,131.9,129.2,127.9,127.0,65.3,49.5,29.9,27.0$, 26.1, $25.5 \mathrm{ppm}$.

HRMS(+ESI): calc'd for $\mathrm{C}_{16} \mathrm{H}_{21} \mathrm{NO}_{3} \mathrm{~S}\left[\mathrm{M}+\mathrm{H}^{+}\right]$308.1315, found 308.1309. 


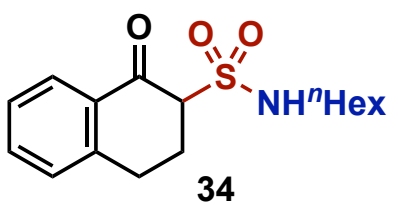

$\beta$-Ketosulfonamide 34 was prepared from alkenyl sulfamate 9 (46 mg, $0.15 \mathrm{mmol}$ ) following general procedure $\mathbf{D}$ (reaction time $=6 \mathrm{~h}$ ). Column chromatography ( $\mathrm{SiO}_{2}$, 5:95 EtOAc:hexanes) afforded 34 as a light-yellow oil (37 mg, 79\%).

$\mathrm{R}_{\mathrm{f}}=0.62(1: 4$ EtOAc:hexanes)

${ }^{1} \mathrm{H}$ NMR $\left(\mathrm{CDCl}_{3}, 400 \mathrm{MHz}\right) \delta: 8.05-8.03(\mathrm{~d}, J=8.7 \mathrm{~Hz}, 1 \mathrm{H}), 7.56-7.52(\mathrm{td}, J=7.5,1.5 \mathrm{~Hz}, 1$ H), 7.37-7.33 (m, 1 H), 7.29-7.27 (d, J=7.9 Hz, $1 \mathrm{H}), 5.00-4.97$ (dd, J=7.7, 4.7 Hz, 1 H), 4.03$3.99(\mathrm{dd}, J=8.5,5.7 \mathrm{~Hz}, 1 \mathrm{H}), 3.34-3.26$ (m, $1 \mathrm{H}), 3.24-3.11(\mathrm{~m}, 2 \mathrm{H}), 3.04-2.97$ (m, $1 \mathrm{H}), 2.74-$ $2.61(\mathrm{~m}, 2 \mathrm{H}), 1.68-1.54$ (m, $2 \mathrm{H}), 1.42-1.25$ (m, $6 \mathrm{H}), 0.91-0.87$ (m, $3 \mathrm{H})$.

${ }^{13} \mathrm{C} \mathrm{NMR}\left(\mathrm{CDCl}_{3}, 126 \mathrm{MHz}\right) \delta: 190.9,144.1,134.9,131.7,129.1,128.1,127.3,64.8,44.0,31.5$, $30.1,27.2,26.4,24.4,22.6,14.1 \mathrm{ppm}$.

HRMS(+ESI): calc'd for $\mathrm{C}_{16} \mathrm{H}_{24} \mathrm{NO}_{3} \mathrm{~S}[\mathrm{M}+\mathrm{H}]^{+} 310.1471$, found 310.1466 .

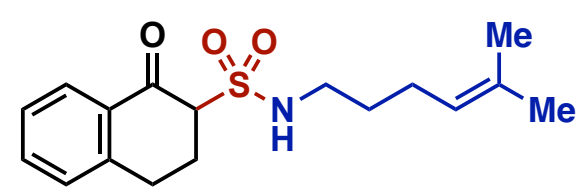

35
$\beta$-Ketosulfonamide 35 was prepared from alkenyl sulfamate 10 (48 $\mathrm{mg}, 0.15 \mathrm{mmol})$ following general procedure D (reaction time $=8 \mathrm{~h})$. Column chromatography $\left(\mathrm{SiO}_{2}\right.$, hexanes to 1:99 $\mathrm{Et}_{2} \mathrm{O}$ :hexanes) afforded $\mathbf{3 5}$ as a light-yellow oil (39

mg, 80\%).

$\mathrm{R}_{\mathrm{f}}=0.50$ (3:7 EtOAc:hexanes)

${ }^{1} \mathrm{H} \mathrm{NMR}\left(\mathrm{CDCl}_{3}, 400 \mathrm{MHz}\right) \delta: 8.06-8.04(\mathrm{dd}, J=7.9,1.5 \mathrm{~Hz}, 1 \mathrm{H}), 7.56-7.52$ (dd, $J=7.5,1.5$ $\mathrm{Hz}, 1 \mathrm{H}), 7.37-7.33$ (t, $J=7.6 \mathrm{~Hz}, 1 \mathrm{H}), 7.29-7.27$ (d, $J=7.7 \mathrm{~Hz}, 1 \mathrm{H}), 5.12-5.07$ (tt, $J=7.3,1.5$ $\mathrm{Hz}, 1 \mathrm{H}), 5.01-4.98(\mathrm{~m}, 1 \mathrm{H}), 4.02-3.99$ (dd, $J=8.6,5.6 \mathrm{~Hz}, 1 \mathrm{H}), 3.30-3.26$ (m, $1 \mathrm{H}), 3.24-3.11$ (m, $2 \mathrm{H}), 3.05-2.97$ (ddd, $J=16.8,8.6,5.0 \mathrm{~Hz}, 1 \mathrm{H}), 2.75-2.61(\mathrm{~m}, 2 \mathrm{H}), 2.11-2.05$ (q, $J=7.3$ $\mathrm{Hz}, 2 \mathrm{H}), 1.71-1.62(\mathrm{~m}, 8 \mathrm{H}) \mathrm{ppm}$.

${ }^{13} \mathrm{C} \mathrm{NMR}\left(\mathrm{CDCl}_{3}, 126 \mathrm{MHz}\right) \delta: \delta 190.9,144.1,134.9,133.0,131.7,129.1,128.1,127.3,123.1$, 64.8, 43.5, 30.2, 27.2, 25.9, 25.2, 24.4, 17.9 ppm.

HRMS(+APCI): calc'd for $\mathrm{C}_{17} \mathrm{H}_{24} \mathrm{NO}_{3} \mathrm{~S}[\mathrm{M}+\mathrm{H}]^{+}$322.1471, found 322.1469. 


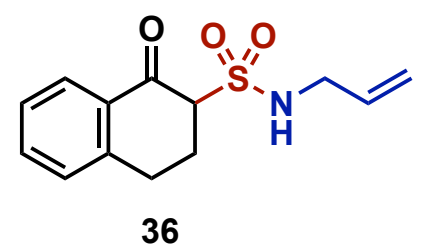

$\beta$-Ketosulfonamide 36 was prepared from alkenyl sulfamate 11 (40 mg, $0.15 \mathrm{mmol}$ ) following general procedure $\mathbf{D}$ (reaction time $=6 \mathrm{~h}$ ). Column chromatography $\left(\mathrm{SiO}_{2}, 10: 90\right.$ to 15:85 EtOAc:hexanes) afforded 36 as a yellow oil (24 mg, 59\%).

$\mathrm{R}_{\mathrm{f}}=0.17$ (1:4 EtOAc:hexanes)

${ }^{1} \mathrm{H}$ NMR $\left(\mathrm{CDCl}_{3}, 400 \mathrm{MHz}\right) \delta: 8.06-8.03(\mathrm{dd}, J=7.9,1.4 \mathrm{~Hz}, 1 \mathrm{H}), 7.56-7.51(\mathrm{td}, J=7.5,1.5$ Hz, $1 \mathrm{H}), 7.37-7.33$ (td, $J=7.6,1.1 \mathrm{~Hz}, 1 \mathrm{H}), 7.29-7.27$ (d, $J=7.8 \mathrm{~Hz}, 1 \mathrm{H}), 5.96-5.83$ (dddd, $J$ $=17.0,10.2,6.6,5.1 \mathrm{~Hz}, 1 \mathrm{H}), 5.37-5.32(\mathrm{dq}, J=17.1,1.5 \mathrm{~Hz}, 1 \mathrm{H}), 5.24-5.19(\mathrm{dq}, J=10.2,1.3$ $\mathrm{Hz}, 1 \mathrm{H}), 5.17-5.11(\mathrm{t}, J=6.4 \mathrm{~Hz}, 1 \mathrm{H}), 4.05-4.01(\mathrm{dd}, J=9.1,5.3 \mathrm{~Hz}, 1 \mathrm{H}), 3.92-3.75(\mathrm{~m}, 2 \mathrm{H})$, 3.32-3.25 (ddd, $J=16.8,6.5,4.6 \mathrm{~Hz}, 1 \mathrm{H}), 3.05-2.98$ (ddd, $J=16.9,9.1,4.8 \mathrm{~Hz}, 1 \mathrm{H}), 2.75-2.60$ $(\mathrm{m}, 2 \mathrm{H}) \mathrm{ppm}$.

${ }^{13} \mathrm{C} \mathrm{NMR}\left(\mathrm{CDCl}_{3}, 126 \mathrm{MHz}\right) \delta: 190.8,144.0,134.9,133.4,131.6,129.1,128.1,127.3,123.1$, 64.8, 43.6, 30.2, 27.2, 25.9, 25.2, 24.4, 17.9 ppm.

HRMS(+APCl): calc'd for $\mathrm{C}_{13} \mathrm{H}_{15} \mathrm{NO}_{3} \mathrm{~S}[\mathrm{M}+\mathrm{H}]^{+} 266.0845$ found 266.0840.

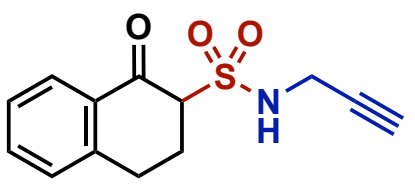

37

$\beta$-Ketosulfonamide 37 was prepared from alkenyl sulfamate 12 (55 $\mathrm{mg}, 0.15 \mathrm{mmol}$ ) following general procedure $\mathrm{D}$ (reaction time $=5 \mathrm{~h}$ ). Column chromatography ( $\mathrm{SiO}_{2}, 10: 90$ to 15:85 EtOAc:hexanes) afforded 37 as a yellow oil (44 mg, 80\%).

$\mathrm{R}_{\mathrm{f}}=0.17$ (1:4 EtOAc:hexanes)

${ }^{1} \mathrm{H}$ NMR $\left(\mathrm{CDCl}_{3}, 400 \mathrm{MHz}\right) 8.08-8.05(\mathrm{dd}, J=7.9,1.4 \mathrm{~Hz}, 1 \mathrm{H}), 7.57-7.53(\mathrm{td}, J=7.5,1.5 \mathrm{~Hz}$, $1 \mathrm{H}), 7.38-7.34$ (t, $J=7.6 \mathrm{~Hz}, 1 \mathrm{H}), 7.29-7.28(\mathrm{~d}, J=7.7 \mathrm{~Hz}, 1 \mathrm{H}), 5.65-5.61(\mathrm{~m}, 1 \mathrm{H}), 4.33-4.29$ $(\mathrm{dd}, J=11.2,4.9 \mathrm{~Hz}, 1 \mathrm{H}), 4.16-4.08$ (m, $2 \mathrm{H}), 3.26-3.19(\mathrm{dt}, J=16.8,4.8 \mathrm{~Hz}, 1 \mathrm{H}), 3.11-3.03$ $(\mathrm{ddd}, J=16.4,10.8,4.5 \mathrm{~Hz}, 1 \mathrm{H}), 2.82-2.74(\mathrm{~m}, 1 \mathrm{H}), 2.67-2.56(\mathrm{~m}, 1 \mathrm{H}), 2.30-2.29(\mathrm{t}, J=2.5$ $\mathrm{Hz}, 1 \mathrm{H}) \mathrm{ppm}$.

${ }^{13} \mathrm{C} \mathrm{NMR}\left(\mathrm{CDCl}_{3}, 126 \mathrm{MHz}\right) \delta: \delta 190.8,143.9,134.9,131.6,129.1,128.2,127.4,78.7,73.7,66.7$, 33.2, 27.7, $24.3 \mathrm{ppm}$.

HRMS(+ESI): calc'd for $\mathrm{C}_{13} \mathrm{H}_{13} \mathrm{NO}_{3} \mathrm{~S}[\mathrm{M}+\mathrm{H}]^{+} 264.0689$ found 264.0685 . 


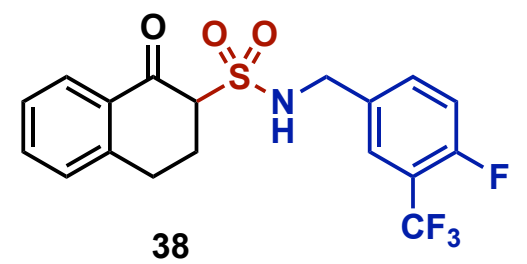

$\beta$-Ketosulfonamide $\mathbf{3 8}$ was prepared from alkenyl sulfamate $\mathbf{1 3}$ (60 $\mathrm{mg}, 0.15 \mathrm{mmol}$ ) following general procedure D (reaction time $=8$ h $)$. Column chromatography $\left(\mathrm{SiO}_{2}, \quad 10: 90\right.$ EtOAc:hexanes) afforded $\mathbf{3 8}$ as a white solid (49 $\mathrm{mg}, 82 \%)$.

$\mathrm{R}_{\mathrm{f}}=0.35$ (3:7 EtOAc:hexanes)

${ }^{1} \mathrm{H}$ NMR $\left(\mathrm{CDCl}_{3}, 400 \mathrm{MHz}\right) \delta: 8.04-8.02(\mathrm{dd}, J=7.9,1.4 \mathrm{~Hz}, 1 \mathrm{H}), 7.64-7.60$ (m, $\left.2 \mathrm{H}\right), 7.57-$ $7.54(\mathrm{td}, J=7.5,1.5 \mathrm{~Hz}, 1 \mathrm{H}), 7.37-7.34(\mathrm{t}, J=7.6 \mathrm{~Hz}, 1 \mathrm{H}), 7.30-7.28(\mathrm{~d}, J=7.8 \mathrm{~Hz}, 1 \mathrm{H}), 7.21-$ 7.18 (t, $J=9.2 \mathrm{~Hz}, 1 \mathrm{H}), 5.50-5.47$ (t, $J=6.6 \mathrm{~Hz}, 1 \mathrm{H}), 4.48-4.33(\mathrm{~m}, 2 \mathrm{H}), 4.00-3.97$ (dd, $J=$ 9.0, 5.3 Hz, $1 \mathrm{H}$ ), 3.34-3.28 (ddd, $J=16.9,6.8,4.7 \mathrm{~Hz}, 1 \mathrm{H}$ ), 3.05-2.98 (ddd, $J=16.8,9.0,4.8$ $\mathrm{Hz}, 1 \mathrm{H}), 2.79-2.58$ (m, $2 \mathrm{H}) \mathrm{ppm}$.

${ }^{13} \mathrm{C}$ NMR $\left(\mathrm{CDCl}_{3}, 126 \mathrm{MHz}\right) \delta: 190.6,160.4$ (d, $\left.J=2.3 \mathrm{~Hz}\right), 158.4-158.3$ (q, $\left.J=2.3 \mathrm{~Hz}\right), 143.9$, 135.0, 133.5 (d, $J=8.6 \mathrm{~Hz}), 133.2$ (d, $J=3.8 \mathrm{~Hz}), 131.4,129.0,128.1,127.3,126.8-126.7$ (qd, $J$ $=4.3,1.6 \mathrm{~Hz}), 125.6-119.0(\mathrm{q}, J=272.2 \mathrm{~Hz}), 119.1-118.2(\mathrm{qd}, J=33.1,12.6 \mathrm{~Hz}), 117.5,117.3$, 65.9, 46.5, 27.1, $24.3 \mathrm{ppm}$.

${ }^{19} \mathrm{~F}$ NMR $\left(\mathrm{CDCl}_{3}, 470 \mathrm{MHz}\right) \delta:-61.8(\mathrm{~d}, J=12.6 \mathrm{~Hz}),-115.7(\mathrm{~m}) \mathrm{ppm}$.

HRMS(+ESI): calc'd for $\mathrm{C}_{18} \mathrm{H}_{15} \mathrm{~F}_{4} \mathrm{NO}_{3} \mathrm{~S}[\mathrm{M}+\mathrm{H}]^{+}$402.0782, found 402.0778 .

mp: $97-100^{\circ} \mathrm{C}$

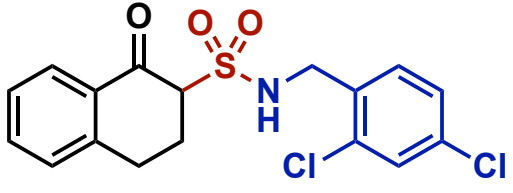

39

$\beta$-Ketosulfonamide 39 was prepared from alkenyl sulfamate 14 (58 $\mathrm{mg}, 0.15 \mathrm{mmol}$ ) following general procedure D (reaction time $=8$ h). Column chromatography $\left(\mathrm{SiO}_{2}, 10: 90\right.$ to $15: 85$ EtOAc:hexanes) afforded 39 as a colorless oil (36 mg, 63\%).

$\mathrm{R}_{\mathrm{f}}=0.33$ (1:4 EtOAc:hexanes)

${ }^{1} \mathrm{H}$ NMR $\left(\mathrm{CDCl}_{3}, 400 \mathrm{MHz}\right) \delta: 8.06-8.04(\mathrm{~d}, J=7.9 \mathrm{~Hz}, 1 \mathrm{H}), 7.57-7.48(\mathrm{~m}, 2 \mathrm{H}), 7.40-7.34$ (m, 2 H), 7.29-7.26 (m, 2 H), 5.61-5.57 (t, $J=6.7$ Hz, 1 H), 4.54-4.41 (m, 2 H), 3.94-3.90 (dd, $J=9.5,5.2 \mathrm{~Hz}, 1 \mathrm{H}), 3.31-3.24$ (dt, $J=16.7,5.4 \mathrm{~Hz}, 1 \mathrm{H}), 3.04-2.97$ (m, $1 \mathrm{H}), 2.76-2.59$ (m, 2 H) $\mathrm{ppm}$.

${ }^{13} \mathrm{C} \mathrm{NMR}\left(\mathrm{CDCl}_{3}, 126 \mathrm{MHz}\right) \delta: 190.6,143.9,135.0,134.7,134.3,133.3,131.6,130.9,129.6$, $129.1,128.2,127.6,127.4,66.3,44.9,27.3,24.4 \mathrm{ppm}$.

HRMS(+APCl): calc'd for $\mathrm{C}_{17} \mathrm{H}_{15} \mathrm{Cl}_{2} \mathrm{NO}_{3} \mathrm{~S}[\mathrm{M}+\mathrm{H}]^{+} 384.0222$ found 384.0215 . 


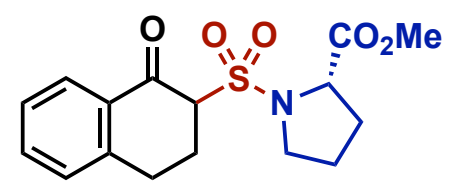

40

$\beta$-Ketosulfonamide 40 was prepared from alkenyl sulfamate 15 (51 $\mathrm{mg}, 0.15 \mathrm{mmol}$ ) following general procedure $\mathbf{D}$ (reaction time $=8$ h). Column chromatography ( $\mathrm{SiO}_{2}, 15: 85$ EtOAc:hexanes) afforded 40 as a dark yellow oil (23 mg, 45\%).

$\mathrm{R}_{\mathrm{f}}=0.25$ (1:4 EtOAc:hexanes)

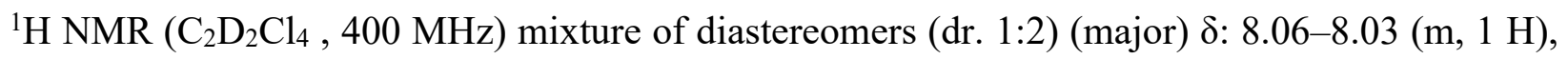
$7.58-7.55$ (t, $J=7.5 \mathrm{~Hz}, 1 \mathrm{H}), 7.39-7.35$ (t, $J=7.6 \mathrm{~Hz}, 1 \mathrm{H}), 7.31-7.29$ (d, $J=7.6 \mathrm{~Hz}, 1 \mathrm{H}), 4.49-$ $4.45(\mathrm{~m}, 1 \mathrm{H}), 4.35-4.32(\mathrm{t}, J=5.6 \mathrm{~Hz}, 1 \mathrm{H}), 3.71(\mathrm{~s}, 3 \mathrm{H}), 3.70-3.48(\mathrm{~m}, 2 \mathrm{H}), 3.45-3.33$ (m, 1 H), 3.03-2.88 (m, $1 \mathrm{H}), 2.76-2.55(\mathrm{~m}, 2 \mathrm{H}), 2.45-2.34(\mathrm{~m}, 1 \mathrm{H}), 2.12-1.98(\mathrm{~m}, 3 \mathrm{H})$; (minor) $\delta$ : 8.06-8.03 (m, $1 \mathrm{H}), 7.58-7.55$ (t, $J=7.5 \mathrm{~Hz}, 1 \mathrm{H}), 7.39-7.35(\mathrm{t}, J=7.6 \mathrm{~Hz}, 1 \mathrm{H}), 7.31-7.29$ (d, $J$ $=7.6 \mathrm{~Hz}, 1 \mathrm{H}), 4.49-4.45(\mathrm{~m}, 1 \mathrm{H}), 4.22-4.19(\mathrm{t}, J=5.7 \mathrm{~Hz}, 1 \mathrm{H}), 3.75(\mathrm{~s}, 3 \mathrm{H}), 3.70-3.48(\mathrm{~m}, 2$ H), 3.45-3.33 (m, $1 \mathrm{H}), 3.03-2.88$ (m, $1 \mathrm{H}), 2.76-2.55$ (m, $2 \mathrm{H}), 2.45-2.34(\mathrm{~m}, 1 \mathrm{H}), 2.12-1.98$ $(\mathrm{m}, 3 \mathrm{H}) \mathrm{ppm}$.

${ }^{13} \mathrm{C} \mathrm{NMR}\left(\mathrm{C}_{2} \mathrm{D}_{2} \mathrm{Cl}_{4}, 126 \mathrm{MHz}\right) \delta: 190.0,189.4,172.8,172.7,143.7,143.6,134.5,134.5,131.5$, $131.5,128.9,128.9,127.7,127.7,127.0,67.1,65.8,61.5,60.5,52.5,52.4,49.9,48.6,31.1,30.7$, 26.4, 26.3, 25.1, 25.0, 24.8, 24.6. ppm.

HRMS(+APCI): calc'd for $\mathrm{C}_{16} \mathrm{H}_{19} \mathrm{NO}_{5} \mathrm{~S}[\mathrm{M}+\mathrm{H}]^{+} 338.1057$, found 336.1050.

Following the method developed by Ley and coworkers (J. Org. Chem. 2012, 77, 11, 5198), 1D gradient NOE NMR of $\mathbf{4 0}$ with an initial selective pulse at $4.3 \mathrm{ppm}$ supports the presence of two diastereomers rather than two rotamers

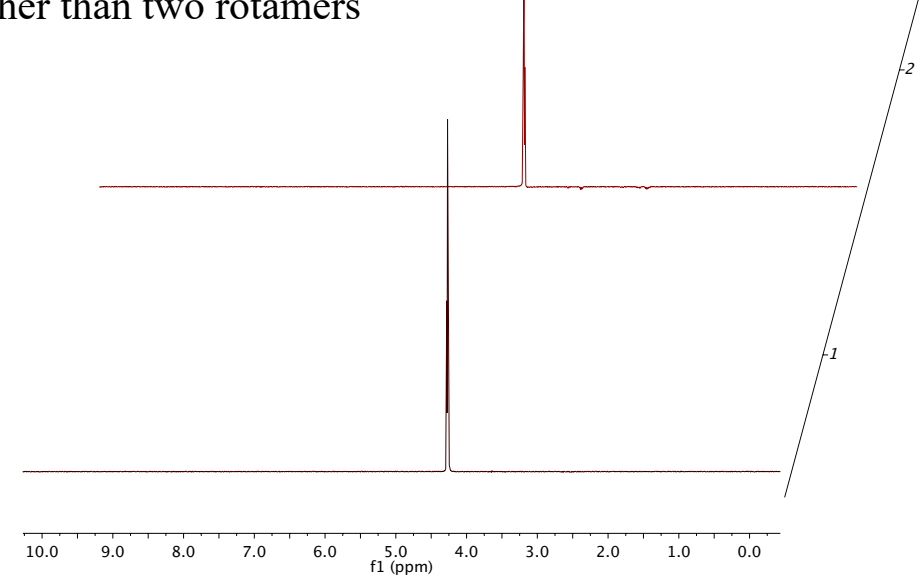

Figure S5. 1D gradient NOE of compound 40. 


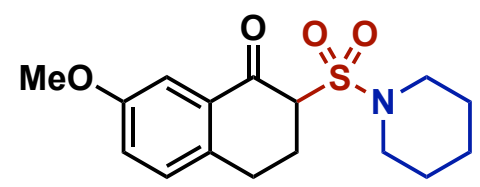

41

$\beta$-Ketosulfonamide 41 was prepared from alkenyl sulfamate 16 (49 $\mathrm{mg}, 0.15 \mathrm{mmol}$ ) following general procedure $\mathbf{D}$ (reaction time $=6$ h). Preparative TLC ( $\mathrm{SiO}_{2}, 30: 70$ EtOAc:hexanes) afforded 41 as brown oil (37 mg, 77\%).

$\mathrm{R}_{\mathrm{f}}=0.47$ (3:7 EtOAc:hexanes)

${ }^{1} \mathrm{H}$ NMR $\left(\mathrm{CDCl}_{3}, 400 \mathrm{MHz}\right) \delta: 7.49(\mathrm{~d}, J=2.9 \mathrm{~Hz}, 1 \mathrm{H}), 7.19-7.16(\mathrm{~d}, J=8.5 \mathrm{~Hz}, 1 \mathrm{H}), 7.11-$ 7.08 (dd, $J=8.5,2.8 \mathrm{~Hz}, 1 \mathrm{H}), 4.00-3.98$ (t, $J=4.8 \mathrm{~Hz}, 1 \mathrm{H}), 3.83$ (s, $3 \mathrm{H}), 3.48-3.29$ (m, $5 \mathrm{H})$, 2.89-2.72 (m, 2 H), 2.55-2.46 (m, $1 \mathrm{H}), 1.68-1.59$ (m, $6 \mathrm{H})$ ppm.

${ }^{13} \mathrm{C} \mathrm{NMR}\left(\mathrm{CDCl}_{3}, 126 \mathrm{MHz}\right) \delta: 190.3,158.6,136.9,132.6,130.4,123.0,109.7,65.5,55.6,47.3$, 26.0, 27.0, 25.5, $24.0 \mathrm{ppm}$.

HRMS(+ESI): calc'd for $\mathrm{C}_{16} \mathrm{H}_{21} \mathrm{NO}_{4} \mathrm{~S}[\mathrm{M}+\mathrm{H}]^{+}$324.1264, found 324.1262.

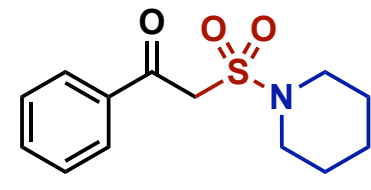

42

$\beta$-Ketosulfonamide 42 was prepared from alkenyl sulfamate 17 (40 mg, $0.15 \mathrm{mmol}$ ) following general procedure $\mathbf{D}$ (reaction time $=8 \mathrm{~h}$ ). Column chromatography ( $\mathrm{SiO}_{2}, 10: 90$ to 15:85 EtOAc:hexanes) afforded $\mathbf{4 2}$ as a white solid (33 mg, 83\%).

The spectroscopic data for this compound were consistent with those reported in the literature (in DMSO-d6, J. Org. Chem. 2019, 84 , 13907).

$\mathrm{R}_{\mathrm{f}}=0.25$ (1:4 EtOAc:hexanes)

${ }^{1} \mathrm{H}$ NMR $\left(\mathrm{CDCl}_{3}, 400 \mathrm{MHz}\right) \delta: 8.05-8.03(\mathrm{~d}, J=7.0 \mathrm{~Hz}, 2 \mathrm{H}), 7.65-7.61(\mathrm{t}, J=7.4 \mathrm{~Hz}, 1 \mathrm{H})$, $7.53-7.49(\mathrm{t}, J=7.7 \mathrm{~Hz}, 2 \mathrm{H}), 4.54(\mathrm{~s}, 2 \mathrm{H}), 3.32-3.29(\mathrm{t}, J=5.1 \mathrm{~Hz}, 4 \mathrm{H}), 1.65-1.54(\mathrm{~m}, 6 \mathrm{H})$ ppm.

${ }^{13} \mathrm{C} \mathrm{NMR}\left(\mathrm{CDCl}_{3}, 126 \mathrm{MHz}\right) \delta: 189.6,136.0,134.4,129.6,128.9,57.6,47.2,25.8,23.8 \mathrm{ppm}$.<smiles>O=C(CS(=O)(=O)N1CCCCC1)c1cc(F)cc(F)c1</smiles>

$\beta$-Ketosulfonamide 43 was prepared from alkenyl sulfamate 19 (46 mg, $0.15 \mathrm{mmol}$ ) following general procedure $\mathbf{D}$ (reaction time $=6 \mathrm{~h}$ ). Column chromatography $\left(\mathrm{SiO}_{2}, 10: 90\right.$ to 15:85 EtOAc:hexanes) afforded 43 as a white solid (23 $\mathrm{mg}, 51 \%)$. 
The spectroscopic data for this compound were consistent with those reported in the literature in DMSO- $d_{6}($ J. Org. Chem. 2019, 84,13907$)$.

$\mathrm{R}_{\mathrm{f}}=0.33$ (1:4 EtOAc:hexanes)

${ }^{1} \mathrm{H}$ NMR $\left(\mathrm{CDCl}_{3}, 400 \mathrm{MHz}\right) \delta: 7.59-7.53$ (m, $\left.2 \mathrm{H}\right), 7.11-7.05$ (tt, $\left.J=8.3,2.3 \mathrm{~Hz}, 1 \mathrm{H}\right), 4.47$ (s, $2 \mathrm{H}), 3.31-3.29$ (m, $4 \mathrm{H}), 1.67-1.56$ (m,6 H) ppm.

${ }^{13} \mathrm{C} \mathrm{NMR}\left(\mathrm{CDCl}_{3}, 126 \mathrm{MHz}\right) \delta: 187.5(\mathrm{t}, J=2.7 \mathrm{~Hz}), 164.4(\mathrm{~d}, J=11.7 \mathrm{~Hz}), 161.9(\mathrm{~d}, J=11.7$ $\mathrm{Hz}), 138.7$ (t, $J=7.9 \mathrm{~Hz}), 112.7$ (d, $J=7.5 \mathrm{~Hz}), 112.5$ (d, $J=7.6 \mathrm{~Hz}), 109.8(\mathrm{t}, J=25.3 \mathrm{~Hz})$, 57.8, 47.3, 25.8, $23.8 \mathrm{ppm}$.

${ }^{19} \mathrm{~F}$ NMR $\left(\mathrm{CDCl}_{3}, 470 \mathrm{MHz}\right) \delta$ : -107.5--107.6 (m) ppm.

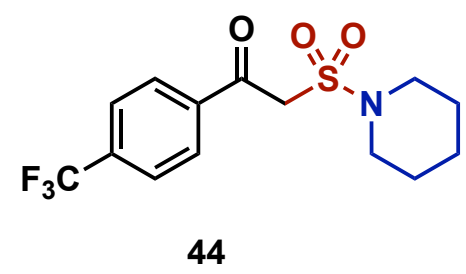

$\beta$-Ketosulfonamide $\mathbf{4 4}$ was prepared from alkenyl sulfamate 20 (50 $\mathrm{mg}, 0.15 \mathrm{mmol}$ ) following general procedure $\mathbf{D}$ (reaction time $=6$ h) using $\left.\left(\operatorname{Ir}\left[\mathrm{dF}\left(\mathrm{CF}_{3}\right) \text { ppy }\right]_{2}\right) \mathrm{dtbpy}\right) \mathrm{PF}_{6}(1 \mathrm{~mol} \%)$ instead of $\operatorname{Ir}(\mathrm{ppy})_{3}$. Column chromatography $\left(\mathrm{SiO}_{2}, 10: 90\right.$ to 15:85 EtOAc:hexanes) afforded $\mathbf{4 4}$ as a white solid (45 $\mathrm{mg}, 90 \%$ ).

The spectroscopic data for this compound were consistent with those reported in the literature (in DMSO- $d_{6}$, J. Org. Chem. 2019, 84,13907$)$.

$\mathrm{R}_{\mathrm{f}}=0.34$ (1:4 EtOAc:hexanes)

${ }^{1} \mathrm{H} \mathrm{NMR}\left(\mathrm{CDCl}_{3}, 400 \mathrm{MHz}\right) \delta: 8.17-8.15(\mathrm{~d}, J=8.1 \mathrm{~Hz}, 2 \mathrm{H}), 7.79-7.77(\mathrm{~d}, J=8.2 \mathrm{~Hz}, 2 \mathrm{H})$, 4.55 (s, $2 \mathrm{H}), 3.32-3.29$ (m, $4 \mathrm{H}), 1.67-1.56$ (m, $6 \mathrm{H}) \mathrm{ppm}$.

${ }^{13} \mathrm{C}$ NMR $\left(\mathrm{CDCl}_{3}, 126 \mathrm{MHz}\right) \delta: 188.9,138.6,135.9-135.2(\mathrm{q}, J=32.8 \mathrm{~Hz}), 130.0,126.1-126.0$ (q, $J=3.7 \mathrm{~Hz}), 126.8-120.3$ (q, $J=272.9 \mathrm{~Hz}), 58.0,47.3,25.8,23.8 \mathrm{ppm}$.

${ }^{19} \mathrm{~F} \mathrm{NMR}\left(\mathrm{CDCl}_{3}, 470 \mathrm{MHz}\right) \delta:-63.7 \mathrm{ppm}$.<smiles></smiles>

$\mathrm{R}_{\mathrm{f}}=0.37$ (1:4 EtOAc:hexanes)

$\beta$-Ketosulfonamide 45 was prepared from alkenyl sulfamate 18 (44 $\mathrm{mg}, 0.15 \mathrm{mmol}$ ) following general procedure $\mathbf{D}$ (reaction time $=6$ h). Column chromatography $\left(\mathrm{SiO}_{2}, 10: 90\right.$ to 15:85 EtOAc:hexanes) afforded $\mathbf{4 5}$ as a white solid (40 mg, 90\%). 
${ }^{1} \mathrm{H}$ NMR $\left(\mathrm{CDCl}_{3}, 400 \mathrm{MHz}\right) \delta: 7.63(\mathrm{~s}, 2 \mathrm{H}), 7.26(\mathrm{~s}, 4 \mathrm{H}), 4.51(\mathrm{~s}, 2 \mathrm{H}), 3.32-3.30$ (t, $J=5.1$ $\mathrm{Hz}, 4 \mathrm{H}), 2.39$ (s, $6 \mathrm{H}), 1.64-1.56$ (m, $6 \mathrm{H}) \mathrm{ppm}$.

${ }^{13} \mathrm{C} \mathrm{NMR}\left(\mathrm{CDCl}_{3}, 126 \mathrm{MHz}\right) \delta: 189.8,138.6,136.1,136.0,127.2,57.5,47.1,25.7,23.7,21.2$ ppm.

HRMS(+ESI): calc'd for $\mathrm{C}_{15} \mathrm{H}_{21} \mathrm{NO}_{3} \mathrm{~S}\left[\mathrm{M}+\mathrm{H}^{+}\right] 296.1315$ found 296.1309. mp: $66-68^{\circ} \mathrm{C}$

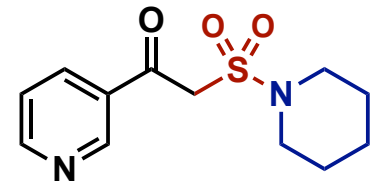

46

$\beta$-Ketosulfonamide 46 was prepared from alkenyl sulfamate 21 (40 mg, $0.15 \mathrm{mmol}$ ) following general procedure $\mathbf{D}$ (reaction time $=16 \mathrm{~h}$ ). Column chromatography ( $\mathrm{SiO}_{2}, 20: 80$ EtOAc:hexanes) afforded 46 as a white solid (33 mg, 83\%).

The spectroscopic data for this compound were consistent with those reported in the literature (in DMSO-d6, J. Org. Chem. 2019, 84,13907$)$.

${ }^{1} \mathrm{H}$ NMR $\left(\mathrm{CDCl}_{3}, 400 \mathrm{MHz}\right) \delta 9.24$ (br.s., $\left.1 \mathrm{H}\right), 8.84-8.83(\mathrm{~d}, J=4.0 \mathrm{~Hz}, 1 \mathrm{H}), 8.34-8.31$ (dt, $J=$ 8.2, $2.0 \mathrm{~Hz}, 1 \mathrm{H}), 7.48-7.45$ (dd, $J=8.1,4.8 \mathrm{~Hz}, 1 \mathrm{H}), 4.53$ (s, $2 \mathrm{H}), 3.31-3.29$ (t, $J=5.1 \mathrm{~Hz}, 4$ H), $1.66-1.56(\mathrm{~m}, 6 \mathrm{H}) \mathrm{ppm}$.

${ }^{13} \mathrm{C} \mathrm{NMR}\left(\mathrm{CDCl}_{3}, 126 \mathrm{MHz}\right) \delta: 188.8,154.5,150.9,136.9,131.4,123.8,57.9,47.3,25.8,23.8$ ppm.

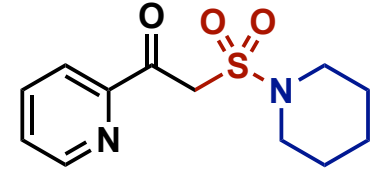

47

$\beta$-Ketosulfonamide 47 was prepared from alkenyl sulfamate 22 (40 $\mathrm{mg}$, $0.15 \mathrm{mmol}$ ) following general procedure $\mathbf{D}$ (reaction time $=16 \mathrm{~h}$ ). Column chromatography ( $\mathrm{SiO}_{2}, 5: 95$ to 20:80 EtOAc:hexanes) afforded 47 as an off white solid (38 mg, 95\%).

$\mathrm{R}_{\mathrm{f}}=0.50$ (3:7 EtOAc:hexanes)

${ }^{1} \mathrm{H}$ NMR $\left(\mathrm{CDCl}_{3}, 400 \mathrm{MHz}\right) \delta: 8.74-8.72$ (m, $\left.1 \mathrm{H}\right), 8.11-8.09$ (dt, $\left.J=7.9,1.1 \mathrm{~Hz}, 1 \mathrm{H}\right), 7.89-$ $7.86(\mathrm{td}, J=7.7,1.7 \mathrm{~Hz}, 1 \mathrm{H}), 7.54-7.51(\mathrm{ddd}, J=7.6,4.7,1.2 \mathrm{~Hz}, 1 \mathrm{H}), 4.94$ (s, 2 H), 3.31-3.29 (t, $J=5.4 \mathrm{~Hz}, 4 \mathrm{H}), 1.65-1.53(\mathrm{~m}, 6 \mathrm{H}) \mathrm{ppm}$.

${ }^{13} \mathrm{C} \mathrm{NMR}\left(\mathrm{CDCl}_{3}, 126 \mathrm{MHz}\right) \delta: 190.9,152.5,149.4,137.4,128.1,122.8,54.5,47.1,25.8,23.9$ ppm.

HRMS(+ESI): calc'd for $\mathrm{C}_{12} \mathrm{H}_{17} \mathrm{~N}_{2} \mathrm{O}_{3} \mathrm{~S}[\mathrm{M}+\mathrm{H}]^{+}$269.0954, found 269.0951. mp: $44-45^{\circ} \mathrm{C}$ 


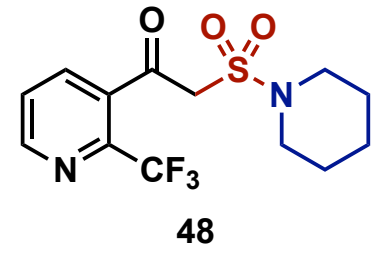

yellow oil (41 mg, 82\%).

$\mathrm{R}_{\mathrm{f}}=0.25$ (3:7 EtOAc:hexanes)

${ }^{1} \mathrm{H}$ NMR $\left(\mathrm{CDCl}_{3}, 400 \mathrm{MHz}\right) \delta: 8.83-8.82(\mathrm{~d}, J=4.5 \mathrm{~Hz}, 1 \mathrm{H}), 8.11-8.10(\mathrm{~d}, J=7.8 \mathrm{~Hz}, 1 \mathrm{H})$, 7.64-7.61 (dd, $J=7.9,4.7 \mathrm{~Hz}, 1 \mathrm{H}), 4.40$ (s, $2 \mathrm{H}), 3.34-3.32$ (t, $J=5.3 \mathrm{~Hz}, 4 \mathrm{H}), 1.70-1.59$ (m, $6 \mathrm{H}) \mathrm{ppm}$.

${ }^{13} \mathrm{C} \mathrm{NMR}\left(\mathrm{CDCl}_{3}, 126 \mathrm{MHz}\right) \delta: 191.5,151.1,144.2-143.4(\mathrm{q}, J=35.0 \mathrm{~Hz}), 137.3,135.0,126.5$, 124.6-118.1 (q, $J=275.3 \mathrm{~Hz}), 61.0-60.9$ (q, $J=2.2 \mathrm{~Hz}), 47.2,25.8,23.7 \mathrm{ppm}$.

${ }^{19} \mathrm{~F}$ NMR $\left(\mathrm{CDCl}_{3}, 470 \mathrm{MHz}\right) \delta$ : $-63.4 \mathrm{ppm}$.

HRMS(+ESI): calc'd for $\mathrm{C}_{13} \mathrm{H}_{15} \mathrm{~F}_{2} \mathrm{~N}_{2} \mathrm{O}_{3} \mathrm{~S}[\mathrm{M}+\mathrm{H}]^{+} 337.0828$, found 337.0822 .

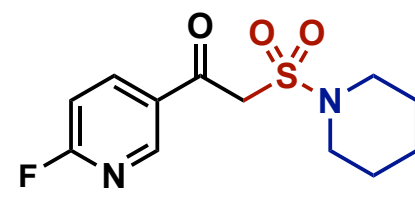

49

$\beta$-Ketosulfonamide 49 was prepared from alkenyl sulfamate 24 (43 $\mathrm{mg}, 0.15 \mathrm{mmol}$ ) following general procedure $\mathbf{D}$ (reaction time $=16$ h). Column chromatography ( $\mathrm{SiO}_{2}, 15: 85$ EtOAc:hexanes) afforded 49 as a yellow solid (40 $\mathrm{mg}, 94 \%$ ).

$\mathrm{R}_{\mathrm{f}}=0.22(3: 7$ EtOAc:hexanes)

${ }^{1} \mathrm{H}$ NMR $\left(\mathrm{CDCl}_{3}, 400 \mathrm{MHz}\right) \delta 8.93-8.92(\mathrm{~d}, J=2.6 \mathrm{~Hz}, 1 \mathrm{H}), 8.48-8.44(\mathrm{ddd}, J=8.6,7.4,2.6$ Hz, 1 H), 7.08-7.06 (m, 1 H), 4.50 (s, 2 H), 3.31-3.29 (m, 4 H), 1.67-1.56 (m, 6 H) ppm.

${ }^{13} \mathrm{C}$ NMR $\left(\mathrm{CDCl}_{3}, 126 \mathrm{MHz}\right) \delta: 187.3,167.3,165.3,151.0-150.9$ (d, $\left.J=16.9 \mathrm{~Hz}\right), 142.5$ (d, $J=$ $9.8 \mathrm{~Hz}), 130.1$ (d, $J=4.4 \mathrm{~Hz}), 110.4-110.1$ (d, $J=37.5 \mathrm{~Hz}), 58.1,47.3,25.8,23.8 \mathrm{ppm}$.

${ }^{19} \mathrm{~F}$ NMR $\left(\mathrm{CDCl}_{3}, 470 \mathrm{MHz}\right) \delta:-58.9(\mathrm{~d}, J=7.4 \mathrm{~Hz})$.

HRMS(+APCI): calc'd for $\mathrm{C}_{12} \mathrm{H}_{16} \mathrm{FN}_{2} \mathrm{O}_{3} \mathrm{~S}[\mathrm{M}+\mathrm{H}]^{+}$287.0860, found 287.0857 . mp: $87-92^{\circ} \mathrm{C}$ 


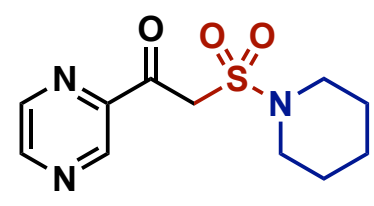

50

$\beta$-Ketosulfonamide $\mathbf{5 0}$ was prepared from alkenyl sulfamate $\mathbf{2 5}$ (40 $\mathrm{mg}$, $0.15 \mathrm{mmol}$ ) following general procedure $\mathbf{D}$ (reaction time $=22 \mathrm{~h}$ ). Preparative TLC (40:60 EtOAc:hexanes) afforded $\mathbf{5 0}$ as a dark yellow oil (26 mg, 64\%).

$\mathrm{R}_{\mathrm{f}}=0.15$ (3:7 EtOAc:hexanes)

${ }^{1} \mathrm{H} \mathrm{NMR}\left(\mathrm{CDCl}_{3}, 400 \mathrm{MHz}\right)$ 8: 9.28 (br.s., $\left.1 \mathrm{H}\right), 8.81$ (d, $\left.J=2.5 \mathrm{~Hz}, 1 \mathrm{H}\right), 8.71-8.70$ (t, $J=2.0$ $\mathrm{Hz}, 1 \mathrm{H}), 4.86$ (s, $2 \mathrm{H}), 3.33-3.30$ (t, $J=5.3 \mathrm{~Hz}, 4 \mathrm{H}), 1.67-1.63$ (m, $4 \mathrm{H}), 1.60-1.56$ (m, $2 \mathrm{H})$ ppm.

${ }^{13} \mathrm{C} \mathrm{NMR}\left(\mathrm{CDCl}_{3}, 126 \mathrm{MHz}\right) \delta:$ 190.5, 148.6, 146.9, 144.5, 143.9, 54.7, 47.2, 25.8, $23.8 \mathrm{ppm}$. HRMS(+ESI): calc'd for $\mathrm{C}_{11} \mathrm{H}_{15} \mathrm{~N}_{3} \mathrm{O}_{3} \mathrm{~S}$ [M+H] 270.0907 , found 270.0906 .

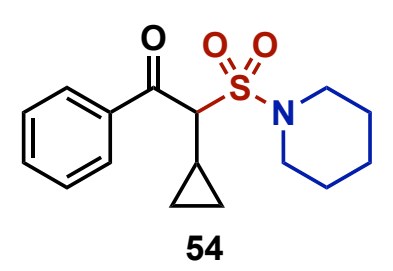

$\beta$-Ketosulfonamide 54 was prepared from alkenyl sulfamate $\mathbf{5 3}$ (92 $\mathrm{mg}$, $0.30 \mathrm{mmol}$ ) following general procedure $\mathbf{D}$ (reaction time $=16 \mathrm{~h}$ ) using $\left(\operatorname{Ir}\left[\mathrm{dF}_{(}\left(\mathrm{CF}_{3}\right) \mathrm{ppy}\right]_{2}\right) \mathrm{dtbpy} \mathrm{PF}_{6}(1 \mathrm{~mol} \%)$ instead of $\operatorname{Ir}(\mathrm{ppy})_{3}$. Column chromatography ( $\mathrm{SiO}_{2}, 10: 90$ EtOAc:hexanes, long column) afforded 54 as a light-yellow solid (10 mg, 11\%).

$\mathrm{R}_{\mathrm{f}}=0.42$ (1:4 EtOAc:hexanes)

${ }^{1} \mathrm{H} \mathrm{NMR}\left(\mathrm{CDCl}_{3}, 400 \mathrm{MHz}\right) \delta$ 7.98-7.95 (m, $\left.2 \mathrm{H}\right), 7.63-7.59$ (m, $\left.1 \mathrm{H}\right), 7.52-7.47$ (dd, $J=8.4$, $7.1 \mathrm{~Hz}, 2 \mathrm{H}), 4.22-4.18$ (d, $J=10.2 \mathrm{~Hz}, 1 \mathrm{H}), 3.42-3.21$ (m, $4 \mathrm{H}), 1.66-1.59$ (m, $7 \mathrm{H}), 0.90-0.76$ (m, $2 \mathrm{H}), 0.70-0.63(\mathrm{~m}, 1 \mathrm{H}), 0.25-0.19$ (ddt, $J=9.6,6.2,4.8 \mathrm{~Hz}, 1 \mathrm{H}) \mathrm{ppm}$.

${ }^{13} \mathrm{C} \mathrm{NMR}\left(\mathrm{CDCl}_{3}, 126 \mathrm{MHz}\right) \delta: 193.9,136.9,134.0,129.1,129.0,73.0,48.0,26.4,24.0,9.9,5.3$, $4.6 \mathrm{ppm}$.

HRMS(+ESI): calc'd for $\mathrm{C}_{16} \mathrm{H}_{21} \mathrm{NO}_{3} \mathrm{~S}[\mathrm{M}+\mathrm{H}]^{+} 308.1315$, found 308.1315 .

mp: $69-75^{\circ} \mathrm{C}$ 
Representative Synthesis of $\beta$-Ketosulfonamides ( $1 \mathrm{mmol} \mathrm{scale})$

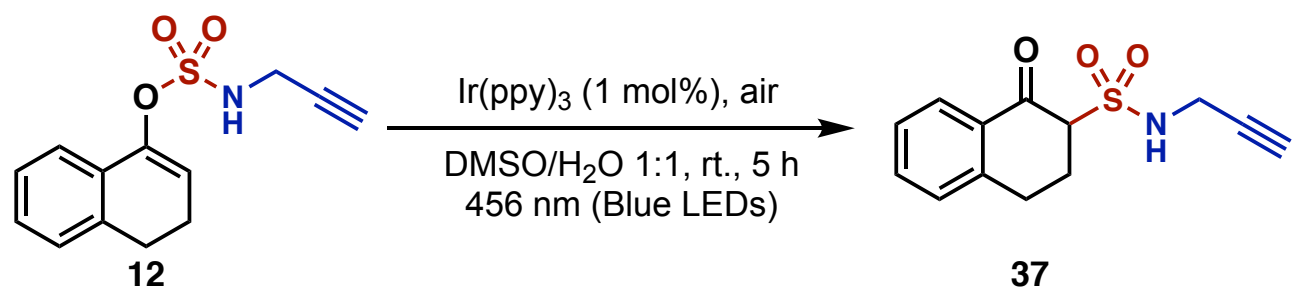

Alkenyl sulfamate 12 (263, $1.0 \mathrm{mmol}, 1.0$ equiv) and $\operatorname{Ir}(\mathrm{ppy})_{3}(6.54 \mathrm{mg}, 0.01 \mathrm{mmol}, 0.01$ equiv) were placed into a $8 \mathrm{~mL}$ vial_(borosilicate glass). DMSO $(2.5 \mathrm{~mL})$ and distilled water $(2.5 \mathrm{~mL})$ were added to the vial. The cloudy solution was irradiated by $456 \mathrm{~nm}$ Kessil lamps (approximately $1 \mathrm{~cm}$ away from the light source) and stirred at room temperature until the full consumption of starting material was observed by TLC. After $5 \mathrm{~h}$, the mixture then was diluted with EtOAc (3 $\mathrm{mL})$, water $(3 \mathrm{~mL})$, and extracted with EtOAc $(3 \times 3 \mathrm{~mL})$ and the combined organic layers were washed with brine $(3 \mathrm{~mL})$ before drying over $\mathrm{MgSO}_{4}$. The solvent was evaporated in vacuo and the crude material was purified via column chromatography $\left(\mathrm{SiO}_{2}, 10: 90\right.$ to 15:85 EtOAc:hexanes) to afford $\mathbf{3 7}$ as a yellow oil (205 $\mathrm{mg}, 78 \%$ ).

Attempt to Induce the 1,3-Rearrangement of 2 Using AIBN as Radical Initiator<smiles>O=S(=O)(OC1=CCCc2ccccc21)N1CCCCC1</smiles>

2

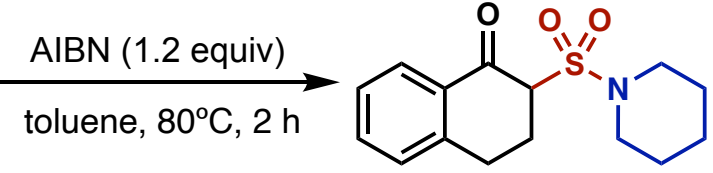

32

To a flame-dried $10 \mathrm{~mL}$ round bottom flask, sulfamate $2(38.0 \mathrm{mg}, 0.13 \mathrm{mmol}, 1.00$ equiv) and AIBN (26.0 mg, $0.16 \mathrm{mmol}, 1.20$ equiv) were added under a nitrogen atmosphere. Anhydrous toluene $(0.65 \mathrm{~mL}, \mathrm{C}=0.2 \mathrm{M})$ was added and the reaction was heated to $80^{\circ} \mathrm{C}$ and monitored by TLC for $2 \mathrm{~h}$. At this point the reaction was cooled to room temperature, the solvent was removed, and the crude material was analyzed by ${ }^{1} \mathrm{H}-\mathrm{NMR}$, which revealed the presence of $\beta$ ketosulfonamide 32 in 30\% yield along with unreacted starting material and several side products. 


\section{Testing the Stability of Alkenyl Sulfamate 3 in Basic and Acidic Aqueous Media}

To a $4 \mathrm{~mL}$ vial the alkenyl sulfamate 3 ( $5 \mathrm{mg}, 0.018 \mathrm{mmol}, 1.0$ equiv) and the aqueous solution (C $=3 \mathrm{M})$ of acid or base $(0.12 \mathrm{~mL}, 0.36 \mathrm{mmol}, 20$ equiv $)$ were added and the reaction was stirred at room temperature for $16 \mathrm{~h}$. Then sat. $\mathrm{NH}_{4} \mathrm{Cl}(5 \mathrm{~mL})$ was added and the resulting mixture was extracted using EtOAc $(3 \times 5 \mathrm{~mL})$. The combined organic phases were washed with brine $(5 \mathrm{~mL})$ and dried over $\mathrm{MgSO}_{4}$. The solvent was evaporated in vacuo and column chromatography $\left(\mathrm{SiO}_{2}\right.$, 10:90 EtOAc:hexanes) afforded pure compound 3.

Table S6. Stability of alkenyl sulfamate 3 under acidic or basic conditions.<smiles>O=S(=O)(OC1=CCCc2ccccc21)N1CCCC1</smiles>

3

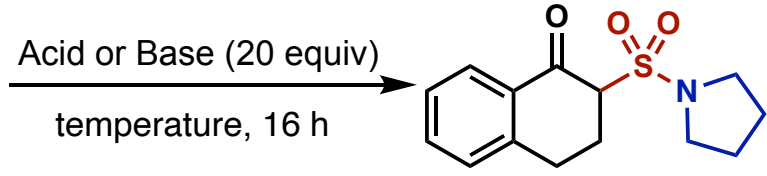

28

\begin{tabular}{cccc} 
Entry & Acid or Base & Temperature & Observations \\
\hline 1 & $a q . \mathrm{NaOH}$ & $\mathrm{rt}$ & full recovery of $\mathbf{3}$ \\
$2^{\mathrm{a}}$ & $a q . \mathrm{NaOH}$ & $\mathrm{rt}$ & full recovery of $\mathbf{3}$ \\
3 & $a q . \mathrm{HCl}$ & $\mathrm{rt}$ & full recovery of $\mathbf{3}$ \\
$4^{\mathrm{a}}$ & $a q . \mathrm{HCl}$ & $\mathrm{rt}$ & $\mathbf{3}(90 \%)+\mathbf{1}(7 \%)$ \\
$5^{\mathrm{b}}$ & $\mathrm{Et}_{3} \mathrm{~N}$ & $80^{\circ} \mathrm{C}$ & full recovery of $\mathbf{3}$
\end{tabular}

$\overline{{ }^{a} \mathrm{MeCN}}(90 \mu \mathrm{L})$ was added in addition to the aqueous solution of acid/base. ${ }^{b} \mathrm{MeCN}(90 \mu \mathrm{L})$ used as the solvent. 


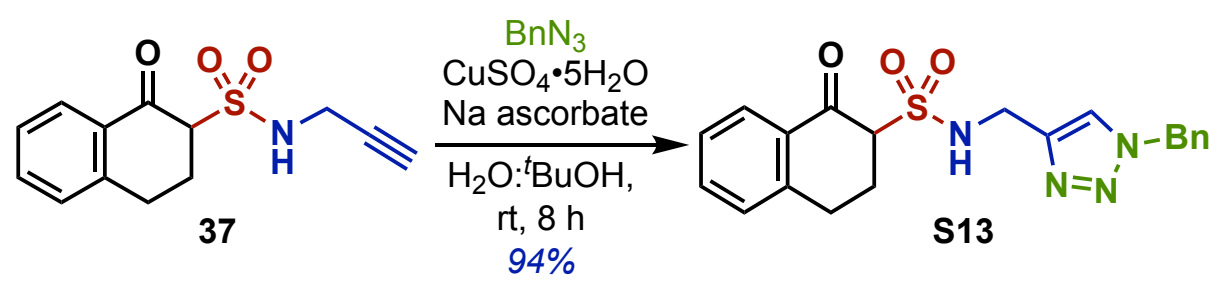

$\beta$-Ketosulfonamide 37 (87 mg, $0.33 \mathrm{mmol}, 1.00$ equiv), $\mathrm{BnN}_{3}$ (S6) (44 mg, $0.33 \mathrm{mmol}$, $\mathrm{CuSO}_{4} \cdot 5 \mathrm{H}_{2} \mathrm{O}(3.4 \mathrm{mg}, 0.0033 \mathrm{mmol}, 0.01$ equiv), and sodium ascorbate $(3.4 \mathrm{mg}, 0.017 \mathrm{mmol}$, 0.05 equiv) were added to a $4 \mathrm{~mL}$ vial under air. ${ }^{t} \mathrm{BuOH}(0.66 \mathrm{~mL})$ and water $(0.66 \mathrm{~mL})$ were added to the vial. The mixture was stirred at room temperature and monitored by TLC until full consumption of the starting material $(8 \mathrm{~h})$. The white suspension was cooled to $0{ }^{\circ} \mathrm{C}$ and diluted with $\mathrm{H}_{2} \mathrm{O}(25 \mathrm{~mL})$ and the white precipitate was collected by filtration and dried under vacuum for $12 \mathrm{~h}$ to afford triazole $\mathbf{S 1 3}$ as a white solid (123 $\mathrm{mg}, 94 \%)$.

${ }^{1} \mathrm{H}$ NMR (DMSO-d $\left.6,400 \mathrm{MHz}\right) \delta: 8.07$ (s, $\left.1 \mathrm{H}\right), 7.94-7.88(\mathrm{~m}, 2 \mathrm{H}), 7.62-7.58(\mathrm{t}, J=7.5 \mathrm{~Hz}, 1$ H), 7.38-7.30 (m, 7 H), 5.59 (s, 2 H), 4.44-4.30 (m, 3 H), 3.22-3.15 (m, 1 H), 2.94-2.87 (m, 1 $\mathrm{H}), 2.48-2.37$ (m, $1 \mathrm{H}) \mathrm{ppm}$.

${ }^{13} \mathrm{C}$ NMR (DMSO- $\left.d_{6}, 126 \mathrm{MHz}\right) \delta: 189.4,144.5,144.0,136.0,134.2,131.6,129.1,128.7,128.1$, $127.9,126.9,126.8,123.4,65.8,52.7,38.2,25.7,24.5 \mathrm{ppm}$.

HRMS(+ESI) calc'd for $\mathrm{C}_{20} \mathrm{H}_{20} \mathrm{~N}_{4} \mathrm{O}_{3} \mathrm{~S}[\mathrm{M}+\mathrm{H}]^{+} 397.1329$, found 397.1328

mp: $105-106^{\circ} \mathrm{C}$ 


\section{Cross-over Experiment}

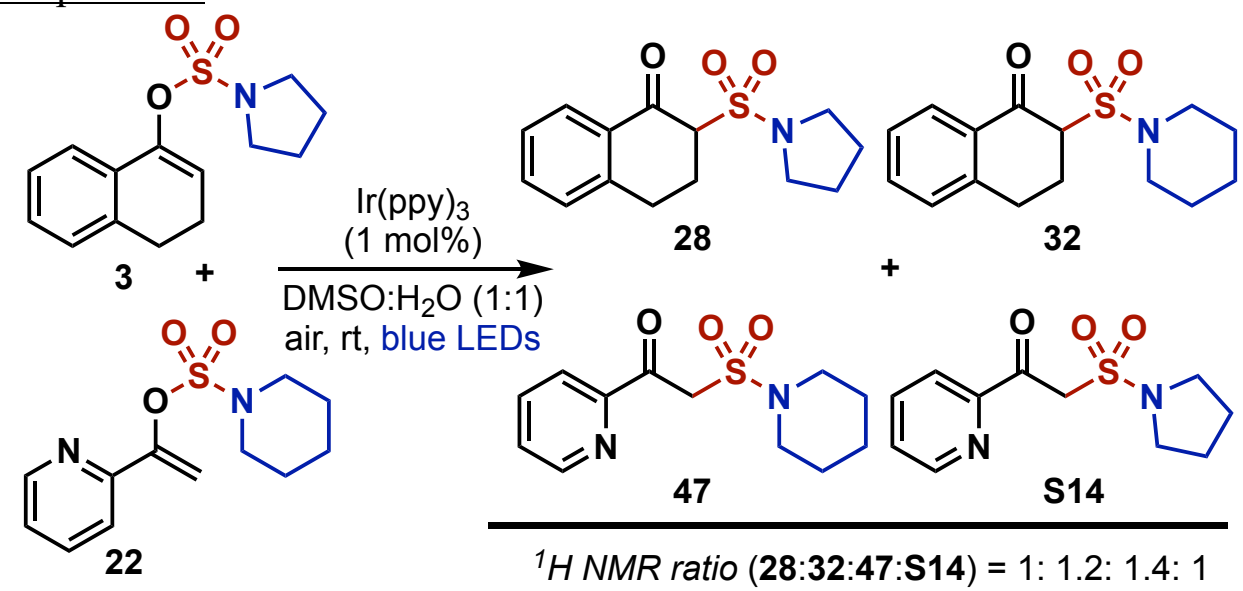

Alkenyl sulfamate 3 (22.3 mg, $0.08 \mathrm{mmol}, 1.00$ equiv), $\operatorname{Ir}(\mathrm{ppy})_{3}(0.5 \mathrm{mg}, 0.0008 \mathrm{mmol}, 0.01$ equiv), and alkenyl sulfamate $22(21.5 \mathrm{mg}, 0.08 \mathrm{mmol}, 1.00$ equiv) were added to a $4 \mathrm{~mL}$ vial (borosilicate glass) open to air. DMSO $(0.2 \mathrm{~mL})$ and water $(0.2 \mathrm{~mL})$ were added to the vial. The cloudy solution was irradiated with a $456 \mathrm{~nm}$ Kessil lamps (approximately $1 \mathrm{~cm}$ away from the light source) and stirred at room temperature for $20 \mathrm{~h}$ (full consumption of both starting materials by TLC analysis). The mixture was the diluted with EtOAc $(3 \mathrm{~mL})$, water $(3 \mathrm{~mL})$, and extracted with EtOAc $(3 \times 3 \mathrm{~mL})$. The combined organic layers were washed with brine $(3 \mathrm{~mL})$, dried over $\mathrm{MgSO}_{4}$, and the solvent was removed under vacuum. Then, the crude material was analyzed by ${ }^{1} \mathrm{H}-\mathrm{NMR}$ (Figure S6), which revealed a distribution of 4 products.

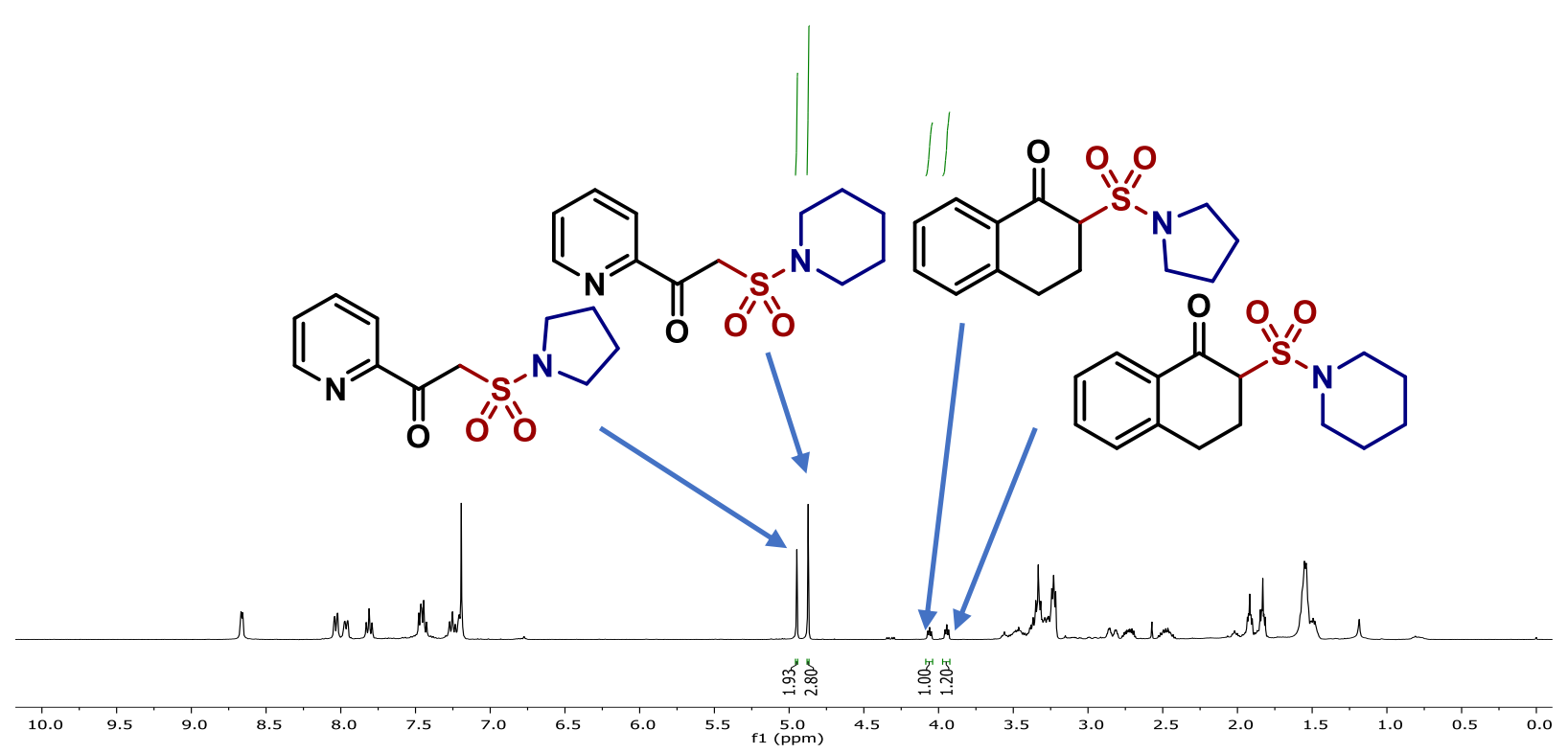

Figure S6. ${ }^{1} \mathrm{H}$ NMR of the crude mixture indicating four different products. 
<smiles>O=S(=O)(OC1=CCCc2ccccc21)N1CCCCC1</smiles>

7

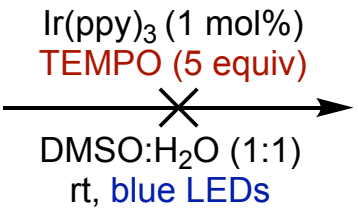

rt, blue LEDs<smiles>O=C1c2ccccc2CCC1S(=O)(=O)N1CCCCC1</smiles>

32

Alkenyl sulfamate 7 (44 mg, $0.15 \mathrm{mmol}, 1.0$ equiv), $\operatorname{Ir}(\mathrm{ppy})_{3}(1.00 \mathrm{mg}, 0.0015 \mathrm{mmol}, 0.01$ equiv), and TEMPO (117 mg, $0.75 \mathrm{mmol}, 5.00$ equiv) were placed into a $4 \mathrm{~mL}$ vial (borosilicate glass). Next, DMSO $(0.38 \mathrm{~mL})$ and water $(0.38 \mathrm{~mL})$ were added to the vial. The cloudy solution was irradiated with a $456 \mathrm{~nm}$ Kessil lamps (approximately $1 \mathrm{~cm}$ away from the light source) and stirred at room temperature for $16 \mathrm{~h}$. Then water $(5 \mathrm{~mL})$ was added and the resulting mixture was extracted using EtOAc $(3 \times 5 \mathrm{~mL})$. The combined organic phases were washed with brine and dried over $\mathrm{MgSO}_{4}$. The solvent was evaporated in vacuo and the crude material was analyzed by ${ }^{1} \mathrm{H}$ NMR indicating full recovery of alkenyl sulfamate 7 .

\section{Radical Clock Experiment}

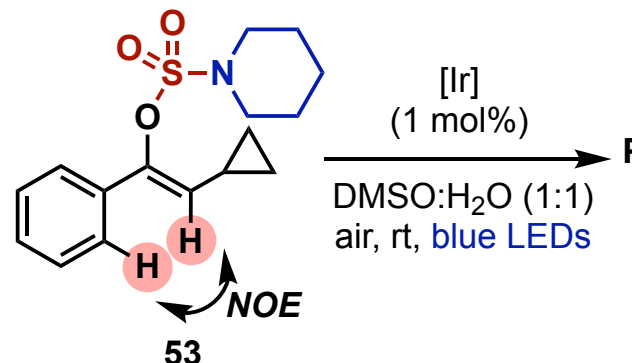<smiles>O=C(c1ccccc1)C(C1CC1)S(=O)(=O)N1CCCCC1</smiles>

54

0.3

|||<smiles>O=S1(=O)OC(c2ccccc2)=CC2CC23CCCCN31</smiles>

53

1 $(Z: E$ ratio $=1: 1)$
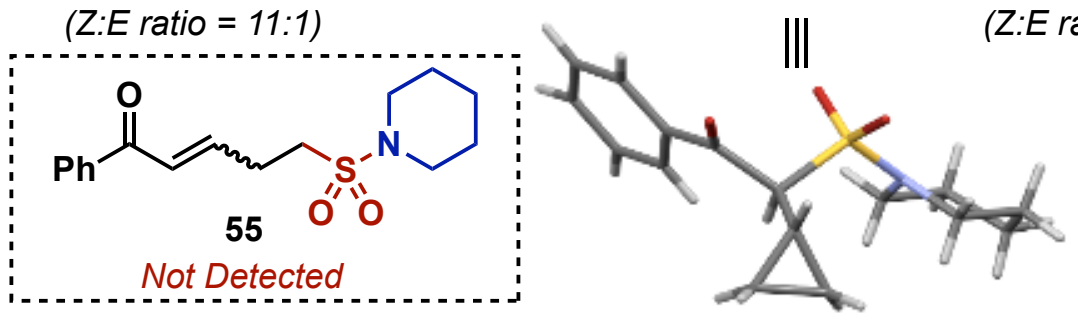
Alkenyl sulfamate 53 (72 mg, $0.23 \mathrm{mmol}, 1.0$ equiv), ( $\left.\operatorname{Ir}\left[\mathrm{dF}\left(\mathrm{CF}_{3}\right) \text { ppy }\right]_{2}(\mathrm{dtbpy})\right) \mathrm{PF}_{6}(2.4 \mathrm{mg}, 0.0023$ mmol, 0.01 equiv) or $\operatorname{Ir}(\text { ppy })_{3}(1.50 \mathrm{mg}, 0.0023 \mathrm{mmol}, 0.01$ equiv), were placed into a $4 \mathrm{~mL}$ vial (borosilicate glass). Next, DMSO $(0.38 \mathrm{~mL})$ and water $(0.38 \mathrm{~mL})$ were added, totalizing a final volume of $0.76 \mathrm{~mL}(\mathrm{C}=0.2 \mathrm{M})$. The cloudy solution was irradiated by $456 \mathrm{~nm}$ Kessil lamps (approximately $1 \mathrm{~cm}$ away from the light source) and stirred at room temperature for $16 \mathrm{~h}$. Then water $(5 \mathrm{~mL})$ was added and the resulting mixture was extracted using EtOAc $(3 \times 5 \mathrm{~mL})$. The combined organic phases were washed with brine and dried over $\mathrm{MgSO}_{4}$. The solvent was evaporated in vacuo and the material was analyzed by ${ }^{1} \mathrm{H}$ NMR indicating recovery of the starting material, isomerization, and 1,3-rearrangement. (Figure S7).

Table S7. Radical clock experiment with two different photocatalysts.

\section{Entry Photocatalyst Product Distribution}

\begin{tabular}{ccc} 
& & $(Z-\mathbf{5 3}: E-\mathbf{5 3}: \mathbf{5 4})$ \\
\hline 1 & $\mathrm{Ir}(\mathrm{ppy})_{3}$ & $1.0: 0.31:$ traces \\
2 & $\left(\mathrm{Ir}\left[\mathrm{dF}\left(\mathrm{CF}_{3}\right) \mathrm{ppy}\right]_{2}(\mathrm{dtbpy})\right) \mathrm{PF}_{6}$ & $1.0: 1.0: 0.35$ \\
\hline
\end{tabular}

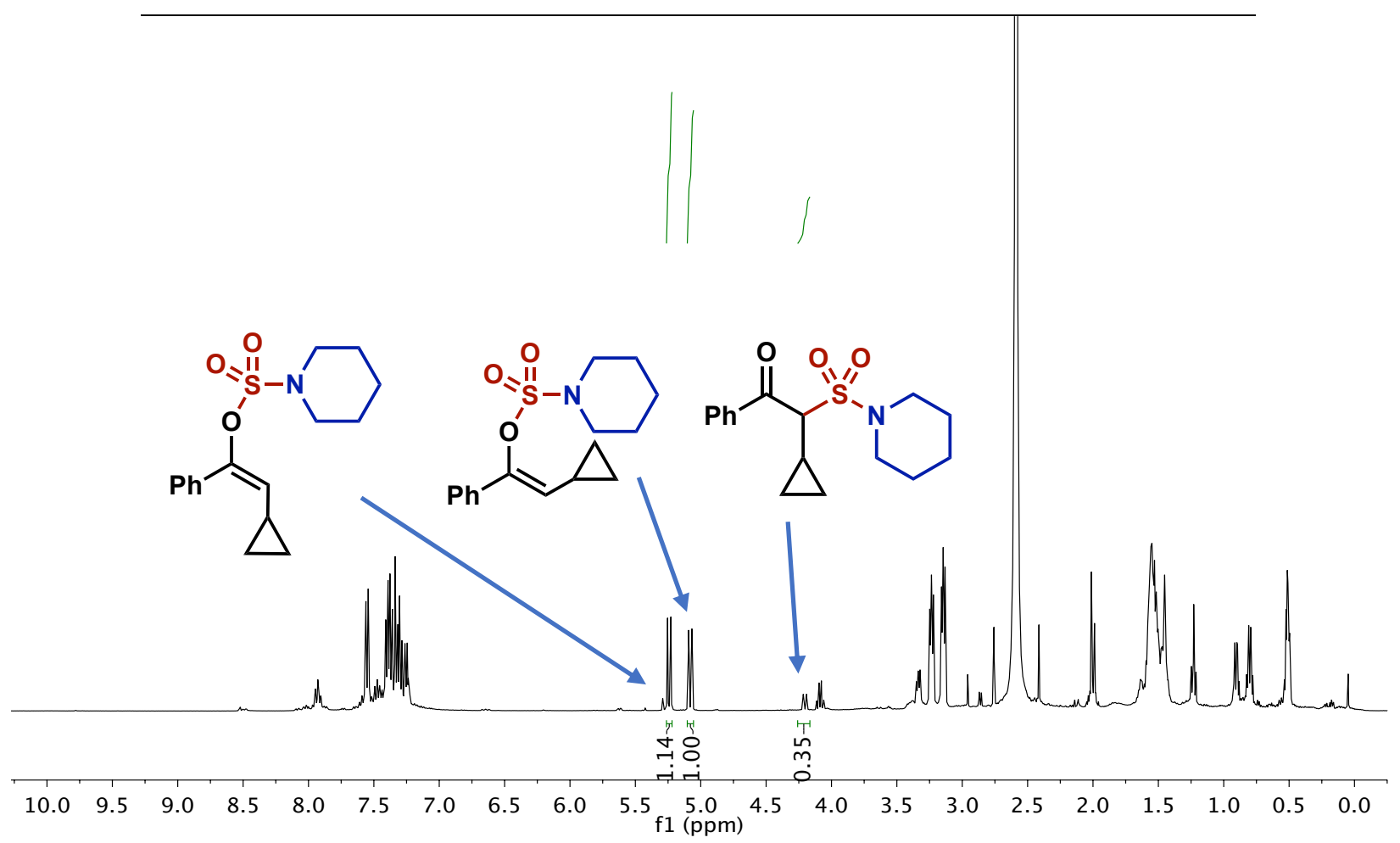

Figure S7. Radical clock experiment: ${ }^{1} \mathrm{H}$ NMR of the crude mixture after irradiation for $16 \mathrm{~h}$. 
Crystallographic Data for Compounds 7 and $\mathbf{5 4}$

Alkenyl Sulfamate 7 (CCDC 2083389)

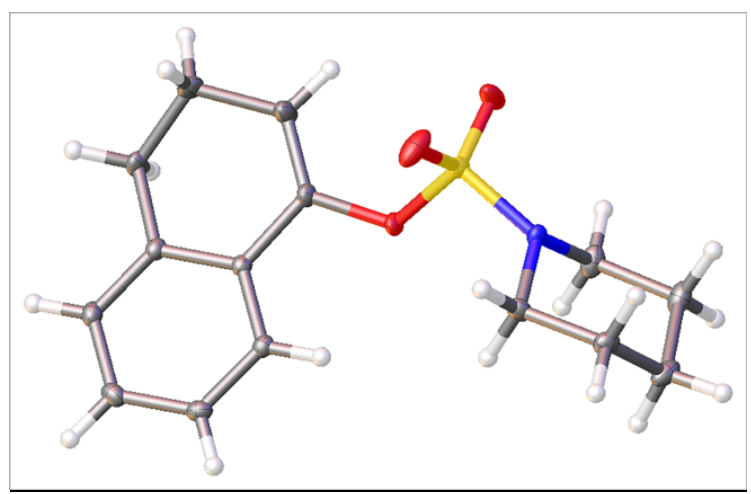

Figure S8. ORTEP drawing of 7 showing thermal ellipsoids at the $50 \%$ probability level.

Table S8. Crystal data and structure refinement for alkenyl sulfamate 7 .

Identification code

Empirical formula

Formula weight

Temperature/K

Crystal system

Space group

$\mathrm{a} / \AA \AA$

$\mathrm{b} / \AA$

$\mathrm{c} / \AA$

$\alpha /{ }^{\circ}$

$\beta /{ }^{\circ}$

$\gamma /{ }^{\circ}$

Volume/ $\AA^{3}$

Z

$\rho_{\text {calc } g} / \mathrm{cm}^{3}$

$\mu / \mathrm{mm}^{-1}$

$\mathrm{F}(000)$

Crystal size $/ \mathrm{mm}^{3}$

Radiation

$2 \Theta$ range for data collection $/{ }^{\circ}$

Index ranges

Reflections collected

Independent reflections

Data/restraints/parameters

Goodness-of-fit on $\mathrm{F}^{2}$
7

$\mathrm{C}_{15} \mathrm{H}_{19} \mathrm{NO}_{3} \mathrm{~S}$

293.37

110

monoclinic

$\mathrm{P} 2{ }_{1} / \mathrm{c}$

8.1961(2)

$8.5339(3)$

20.4844(6)

90

96.782(3)

90

1422.75(7)

4

1.370

0.234

624.0

$0.1 \times 0.1 \times 0.1$

$\operatorname{MoK} \alpha(\lambda=0.71073)$

4.004 to 66.202

$-12 \leq \mathrm{h} \leq 12,-13 \leq \mathrm{k} \leq 13,-31 \leq 1 \leq 31$

28995

$5080\left[\mathrm{R}_{\text {int }}=0.0535, \mathrm{R}_{\text {sigma }}=0.0458\right]$

$5080 / 0 / 181$

1.054 
Final $\mathrm{R}$ indexes $[\mathrm{I}>=2 \sigma(\mathrm{I})] \quad \mathrm{R}_{1}=0.0441, \mathrm{wR}_{2}=0.0965$

Final $\mathrm{R}$ indexes [all data] $\quad \mathrm{R}_{1}=0.0718, \mathrm{wR}_{2}=0.1072$

Largest diff. peak/hole / e $\AA^{-3} \quad 0.43 /-0.56$

Table S9. Fractional Atomic Coordinates $\left(\times 10^{4}\right)$ and Equivalent Isotropic Displacement Parameters $\left(\AA^{2} \times 10^{3}\right)$ for $7 \mathrm{U}_{\text {eq }}$ is defined as $1 / 3$ of of the trace of the orthogonalised $\mathrm{U}_{\text {IJ }}$ tensor.

\begin{tabular}{lrrrr} 
Atom & \multicolumn{3}{c}{$\boldsymbol{y}$} & \multicolumn{1}{c}{$\boldsymbol{U}(\mathbf{e q})$} \\
S1 & $6966.5(4)$ & $7046.0(4)$ & $1852.4(2)$ & $14.19(8)$ \\
O1 & $6826.1(12)$ & $5508.9(11)$ & $2109.6(5)$ & $27.9(2)$ \\
O2 & $8205.4(11)$ & $7359.4(14)$ & $1436.8(5)$ & $27.4(2)$ \\
O3 & $7220.5(11)$ & $8250.3(11)$ & $2458.5(4)$ & $15.85(18)$ \\
N1 & $5219.9(12)$ & $7602.9(12)$ & $1484.9(5)$ & $12.8(2)$ \\
C1 & $7218.2(15)$ & $1801.6(6)$ & $15.4(2)$ \\
C2 & $3729.5(15)$ & $7236.1(15)$ & $1276.0(6)$ & $16.6(2)$ \\
C3 & $2250.6(15)$ & $8838.1(15)$ & $942.6(7)$ & $20.2(3)$ \\
C4 & $2077.0(16)$ & $9238.4(15)$ & $647.0(7)$ & $19.9(3)$ \\
C5 & $3648.5(16)$ & $9166.6(15)$ & $1164.6(6)$ & $18.7(3)$ \\
C6 & $5147.4(16)$ & $7923.1(14)$ & $2997.3(6)$ & $13.6(2)$ \\
C7 & $8420.5(15)$ & $8138.3(13)$ & $3635.8(6)$ & $11.3(2)$ \\
C8 & $7812.1(14)$ & $8298.8(14)$ & $3696.3(6)$ & $12.5(2)$ \\
C9 & $6133.3(14)$ & $8507.6(14)$ & $4314.6(6)$ & $15.1(2)$ \\
C10 & $5620.7(15)$ & $8528.1(14)$ & $4872.7(6)$ & $15.7(2)$ \\
C11 & $8366.7(14)$ & $4813.3(6)$ & $14.4(2)$ \\
C12 & $8764.2(15)$ & $8185.8(13)$ & $4197.2(6)$ & $12.6(2)$ \\
C13 & $8436.2(15)$ & $8137.3(16)$ & $4107.8(6)$ & $17.0(2)$ \\
C14 & $8977.7(14)$ & $7098.7(17)$ & $3530.3(6)$ & $20.5(3)$ \\
C15 & $7457.6(17)$ & $2929.6(6)$ & $20.9(3)$ \\
& $10786.8(14)$ & & &
\end{tabular}

Table S10. Anisotropic Displacement Parameters $\left(\AA^{2} \times 10^{3}\right)$ for 7 . The Anisotropic displacement factor exponent takes the form: $-2 \pi^{2}\left[\mathrm{~h}^{2} \mathrm{a}^{* 2} \mathrm{U}_{11}+2 \mathrm{hka} \mathrm{b}^{*} \mathrm{U}_{12}+\ldots\right]$.

\begin{tabular}{lrrrrrr} 
Atom & \multicolumn{1}{c}{$\mathbf{U}_{\mathbf{1 1}}$} & \multicolumn{1}{c}{$\mathbf{U}_{\mathbf{2 2}}$} & \multicolumn{1}{c}{$\mathbf{U}_{\mathbf{3 3}}$} & \multicolumn{1}{c}{$\mathbf{U}_{\mathbf{2 3}}$} & \multicolumn{1}{c}{$\mathbf{U}_{\mathbf{1 3}}$} & \multicolumn{1}{c}{$\mathbf{U}_{\mathbf{1 2}}$} \\
$\mathrm{S} 1$ & $10.92(14)$ & $18.77(15)$ & $12.18(14)$ & $-4.42(11)$ & $-1.56(10)$ & $1.69(11)$ \\
$\mathrm{O} 1$ & $24.5(5)$ & $17.0(5)$ & $38.1(6)$ & $0.6(4)$ & $-13.3(4)$ & $1.9(4)$ \\
$\mathrm{O} 2$ & $11.6(4)$ & $57.5(7)$ & $13.2(4)$ & $-7.3(4)$ & $1.6(4)$ & $2.9(4)$ \\
$\mathrm{O} 3$ & $15.0(4)$ & $21.8(4)$ & $9.8(4)$ & $-4.7(3)$ & $-3.0(3)$ & $3.8(3)$ \\
$\mathrm{N} 1$ & $9.6(4)$ & $16.6(5)$ & $11.8(5)$ & $0.2(4)$ & $-0.9(4)$ & $-0.8(4)$ \\
$\mathrm{C} 1$ & $12.9(5)$ & $19.7(6)$ & $14.1(5)$ & $-1.2(4)$ & $3.6(4)$ & $-2.1(4)$ \\
C2 & $11.0(5)$ & $19.7(6)$ & $18.9(6)$ & $-2.1(5)$ & $1.1(4)$ & $-0.8(4)$ \\
C3 & $16.0(6)$ & $17.1(6)$ & $25.6(7)$ & $-4.3(5)$ & $-4.8(5)$ & $4.0(5)$ \\
C4 & $19.9(6)$ & $16.2(6)$ & $21.5(6)$ & $4.3(5)$ & $-5.8(5)$ & $-1.9(5)$ \\
C5 & $18.5(6)$ & $16.9(6)$ & $19.3(6)$ & $3.4(5)$ & $-3.2(5)$ & $-5.2(5)$ \\
C6 & $11.9(5)$ & $17.3(6)$ & $10.7(5)$ & $-1.8(4)$ & $-1.7(4)$ & $-0.3(4)$
\end{tabular}




$\begin{array}{lrrrrrr}\text { C7 } & 11.7(5) & 10.4(5) & 11.6(5) & -0.6(4) & 0.4(4) & 0.1(4) \\ \text { C8 } & 12.1(5) & 11.9(5) & 13.0(5) & 0.0(4) & -0.6(4) & 0.2(4) \\ \text { C9 } & 13.3(5) & 15.0(5) & 17.5(6) & -0.1(4) & 4.0(4) & 1.4(4) \\ \text { C10 } & 19.3(6) & 15.4(6) & 13.2(5) & 0.0(4) & 4.9(5) & 0.5(5) \\ \text { C11 } & 15.8(6) & 14.4(5) & 12.3(5) & -0.5(4) & -1.5(4) & 0.9(4) \\ \text { C12 } & 12.2(5) & 12.0(5) & 12.9(5) & -1.1(4) & -1.1(4) & 0.4(4) \\ \text { C13 } & 10.5(5) & 25.7(7) & 13.8(5) & -1.2(5) & -2.2(4) & -0.2(5) \\ \text { C14 } & 10.7(5) & 31.3(7) & 19.0(6) & -4.1(5) & -0.2(5) & 4.0(5) \\ \text { C15 } & 13.6(6) & 35.7(7) & 13.1(6) & -5.6(5) & 0.3(5) & 2.9(5)\end{array}$

Table S11. Bond Lengths for 7.

\begin{tabular}{|c|c|c|c|c|c|c|}
\hline \multicolumn{2}{|c|}{ Atom } & \multirow[t]{2}{*}{ Atom } & \multirow{2}{*}{$\begin{array}{c}\text { Length/§ } \\
1.4233(11)\end{array}$} & Atom & Atom & \multirow{2}{*}{$\begin{array}{l}\text { Length/A } \\
1.4654(16)\end{array}$} \\
\hline $\mathrm{S} 1$ & $\mathrm{O} 1$ & & & C6 & $\mathrm{C} 7$ & \\
\hline S1 & $\mathrm{O} 2$ & & $1.4253(10)$ & C6 & $\mathrm{C} 15$ & $1.3354(17)$ \\
\hline S1 & $\mathrm{O} 3$ & & $1.6062(9)$ & $\mathrm{C} 7$ & $\mathrm{C} 8$ & $1.4027(16)$ \\
\hline S1 & N1 & & $1.6085(11)$ & $\mathrm{C} 7$ & $\mathrm{C} 12$ & $1.4061(16)$ \\
\hline $\mathrm{O} 3$ & C6 & & $1.4166(14)$ & $\mathrm{C} 8$ & C9 & $1.3921(16)$ \\
\hline N1 & $\mathrm{C} 1$ & & $1.4860(15)$ & C9 & $\mathrm{C} 10$ & $1.3902(18)$ \\
\hline N1 & $\mathrm{C} 5$ & & $1.4851(16)$ & $\mathrm{C} 10$ & $\mathrm{C} 11$ & $1.3969(17)$ \\
\hline $\mathrm{C} 1$ & $\mathrm{C} 2$ & & $1.5234(18)$ & $\mathrm{C} 11$ & $\mathrm{C} 12$ & $1.3949(16)$ \\
\hline $\mathrm{C} 2$ & $\mathrm{C} 3$ & & $1.5273(18)$ & $\mathrm{C} 12$ & $\mathrm{C} 13$ & $1.5160(16)$ \\
\hline $\mathrm{C} 3$ & $\mathrm{C} 4$ & & $1.5253(18)$ & $\mathrm{C} 13$ & $\mathrm{C} 14$ & $1.5296(17)$ \\
\hline $\mathrm{C} 4$ & $\mathrm{C} 5$ & & $1.5263(18)$ & $\mathrm{C} 14$ & $\mathrm{C} 15$ & $1.5035(18)$ \\
\hline
\end{tabular}

Table S12. Bond Angles for 7.

\begin{tabular}{llllllll} 
Atom Atom & Atom & \multicolumn{1}{c}{ Angle/ } & \multicolumn{2}{c}{ Atom } & Atom & Atom & Angle/ \\
O1 & S1 & O2 & $119.24(7)$ & C15 & C6 & O3 & $123.41(11)$ \\
O1 & S1 & O3 & $108.20(6)$ & C15 & C6 & C7 & $123.48(11)$ \\
O1 & S1 & N1 & $109.82(6)$ & C8 & C7 & C6 & $122.13(11)$ \\
O2 & S1 & O3 & $107.77(6)$ & C8 & C7 & C12 & $120.24(11)$ \\
O2 & S1 & N1 & $108.81(6)$ & C12 & C7 & C6 & $117.63(10)$ \\
O3 & S1 & N1 & $101.52(5)$ & C9 & C8 & C7 & $119.83(11)$ \\
C6 & O3 & S1 & $119.39(8)$ & C10 & C9 & C8 & $120.21(11)$ \\
C1 & N1 & S1 & $117.54(8)$ & C9 & C10 & C11 & $120.01(11)$ \\
C5 & N1 & S1 & $117.34(8)$ & C12 & C11 & C10 & $120.66(11)$ \\
C5 & N1 & C1 & $113.54(10)$ & C7 & C12 & C13 & $118.70(10)$ \\
N1 & C1 & C2 & $108.40(10)$ & C11 & C12 & C7 & $119.02(11)$ \\
C1 & C2 & C3 & $110.45(10)$ & C11 & C12 & C13 & $122.18(11)$ \\
C4 & C3 & C2 & $109.85(10)$ & C12 & C13 & C14 & $112.26(10)$ \\
C3 & C4 & C5 & $111.30(11)$ & C15 & C14 & C13 & $110.91(11)$ \\
N1 & C5 & C4 & $109.26(10)$ & C6 & C15 & C14 & $119.67(11)$ \\
O3 & C6 & C7 & $113.11(10)$ & & & &
\end{tabular}


Table S13. Hydrogen Atom Coordinates $\left(\AA \times 10^{4}\right)$ and Isotropic Displacement Parameters $\left(\AA^{2} \times 10^{3}\right)$ for 7 .

\begin{tabular}{lrrrrr}
\multicolumn{1}{c}{ Atom } & \multicolumn{1}{c}{$\boldsymbol{x}$} & $\boldsymbol{y}$ & \multicolumn{2}{c}{$\mathbf{U ( e q )}$} \\
H1A & 3578.44 & 7998.29 & 2147.15 & 19 \\
H1B & 3848.81 & 6170.09 & 2008.94 & 19 \\
H2A & 1241.93 & 6996.4 & 1479.37 & 20 \\
H2B & 2385.74 & 6419.12 & 943.53 & 20 \\
H3A & 1135.7 & 8824.11 & 592.09 & 24 \\
H3B & 1862.57 & 9646.62 & 1268.4 & 24 \\
H4A & 3552.55 & 10304.43 & 455.65 & 24 \\
H4B & 3794.15 & 8491.58 & 288.57 & 24 \\
H5A & 6159.3 & 9346.44 & 954.85 & 22 \\
H5B & 5070.74 & 9994.81 & 1497.99 & 22 \\
H8 & 5348.19 & 8265.21 & 3316.33 & 15 \\
H9 & 4485.86 & 8636.42 & 4355.27 & 18 \\
H10 & 6408.42 & 8651.91 & 5294.59 & 19 \\
H11 & 9212.42 & 8380.31 & 5196.3 & 17 \\
H13A & 11417.42 & 7736.65 & 4515.66 & 20 \\
H13B & 11172.51 & 9214.69 & 4033.05 & 20 \\
H14A & 12265.7 & 7272.38 & 3431.69 & 25 \\
H14B & 11017.63 & 5983.74 & 3651.57 & 25 \\
H15 & 10286.58 & 7353.72 & 2504.08 & 25
\end{tabular}

Experimental

Single crystals of $\mathrm{C}_{15} \mathrm{H}_{19} \mathrm{NO}_{3} \mathrm{~S}$ (7) were grown through slow evaporation of a concentrated solution of 7 in $\mathrm{CDCl}_{3}$. A suitable crystal was selected and analyzed on a Bruker Venture Photon 2 Microsource diffractometer. The crystal was kept at $110 \mathrm{~K}$ during data collection. Using Olex2 [1], the structure was solved with the ShelXT [2] structure solution program using Intrinsic Phasing and refined with the ShelXL [3] refinement package using Least Squares minimisation.

1. Dolomanov, O.V., Bourhis, L.J., Gildea, R.J, Howard, J.A.K. \& Puschmann, H. (2009), J. Appl. Cryst. 42, 339-341.

2. Sheldrick, G.M. (2015). Acta Cryst. A71, 3-8.

3. Sheldrick, G.M. (2015). Acta Cryst. C71, 3-8.

Crystal structure determination of 7

Crystal Data for $\mathrm{C}_{15} \mathrm{H}_{19} \mathrm{NO}_{3} \mathrm{~S}(M=293.37 \mathrm{~g} / \mathrm{mol})$ : monoclinic, space group $\mathrm{P} 21 / \mathrm{c}$ (no. 14), $a=8.1961(2) \AA, b=8.5339(3) \AA, c=20.4844(6) \AA, \beta=96.782(3)^{\circ}, V=1422.75(7) \AA^{3}, Z=4, T=$ $110 \mathrm{~K}, \mu(\mathrm{MoK} \alpha)=0.234 \mathrm{~mm}^{-1}$, Dcalc $=1.370 \mathrm{~g} / \mathrm{cm}^{3}, 28995$ reflections measured $\left(4.004^{\circ} \leq 2 \Theta \leq\right.$ $\left.66.202^{\circ}\right), 5080$ unique $\left(R_{\text {int }}=0.0535, R_{\text {sigma }}=0.0458\right)$ which were used in all calculations. The final $R_{1}$ was 0.0441 (I $\left.>2 \sigma(\mathrm{I})\right)$ and $w R_{2}$ was 0.1072 (all data).

Refinement model description

Number of restraints - 0 , number of constraints - unknown. 
Details:

1. Fixed Uiso

At 1.2 times of:

All C( $\mathrm{H})$ groups, All $\mathrm{C}(\mathrm{H}, \mathrm{H})$ groups

2.a Secondary $\mathrm{CH} 2$ refined with riding coordinates:

C1(H1A,H1B), C2(H2A,H2B), C3(H3A,H3B), C4(H4A,H4B), C5(H5A,H5B), C13(H13A, H13B), C14(H14A,H14B)

2.b Aromatic/amide $\mathrm{H}$ refined with riding coordinates:

C8(H8), C9(H9), C10(H10), C11(H11), C15(H15)

$\beta$-Ketosulfonamide 54 (CCDC 2083388)

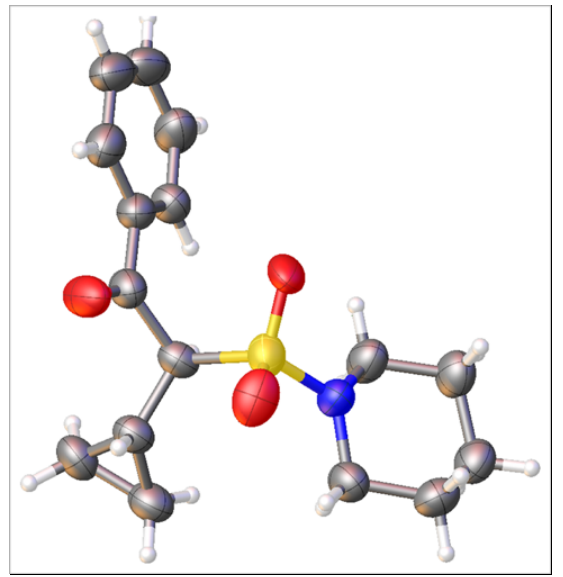

Figure S9. ORTEP drawing of $\mathbf{5 4}$ showing thermal ellipsoids at the $50 \%$ probability level.

Table S14. Crystal data and structure refinement for $\beta$-ketosulfonamide 54.

Identification code

54

Empirical formula

$\mathrm{C}_{16} \mathrm{H}_{21} \mathrm{NO}_{3} \mathrm{~S}$

Formula weight

307.40

Temperature/K

110

Crystal system

monoclinic

Space group

$\mathrm{P} 21 / \mathrm{n}$

$\mathrm{a} / \AA$

5.4513(3)

$\mathrm{b} / \AA \AA$

28.2495(18)

$\mathrm{c} / \AA$

$10.4075(8)$

$\alpha /{ }^{\circ}$

90

$\beta /{ }^{\circ}$

$\gamma /{ }^{\circ}$

$100.332(7)$

Volume $/ \AA^{3}$

90

Z

1576.73(18)

4 


$\begin{array}{ll}\rho_{\text {calcg }} / \mathrm{cm}^{3} & 1.295 \\ \mu / \mathrm{mm}^{-1} & 1.904 \\ \mathrm{~F}(000) & 656.0 \\ \text { Crystal size } / \mathrm{mm}^{3} & 0.2 \times 0.05 \times 0.05 \\ \text { Radiation } & \mathrm{CuK} \alpha(\lambda=1.54184) \\ 2 \Theta \text { range for data collection } /{ }^{\circ} & 6.258 \text { to } 158.368 \\ \text { Index ranges } & -6 \leq \mathrm{h} \leq 5,-35 \leq \mathrm{k} \leq 35,-13 \leq 1 \leq 13 \\ \text { Reflections collected } & 36657 \\ \text { Independent reflections } & 3355\left[\mathrm{R}_{\text {int }}=0.1305, \mathrm{R}_{\text {sigma }}=0.0510\right] \\ \text { Data/restraints/parameters } & 3355 / 0 / 190 \\ \text { Goodness-of-fit on } \mathrm{F}^{2} & 1.035 \\ \text { Final R indexes }[\mathrm{I}>=2 \sigma(\mathrm{I})] & \mathrm{R}_{1}=0.1395, \mathrm{wR}_{2}=0.2930 \\ \text { Final R indexes }[\text { all data] } & \mathrm{R}_{1}=0.1613, \mathrm{wR}_{2}=0.3043 \\ \text { Largest diff. peak/hole } / \mathrm{e} \AA^{-3} & 0.99 /-0.51\end{array}$

Table S15. Fractional Atomic Coordinates $\left(\times 10^{4}\right)$ and Equivalent Isotropic Displacement Parameters $\left(\AA^{2} \times 10^{3}\right)$ for 54. $U_{\text {eq }}$ is defined as $1 / 3$ of of the trace of the orthogonalised $U_{\text {IJ }}$ tensor.

\begin{tabular}{lrrrr} 
Atom & \multicolumn{2}{c}{$\boldsymbol{y}$} & \multicolumn{1}{c}{ U(eq) } \\
S1 & \multicolumn{1}{c}{$\boldsymbol{z}$} & $6724.5(18)$ & $57.8(5)$ \\
O1 & $5230(4)$ & $6143.5(7)$ & $7078(5)$ & $74.0(17)$ \\
O2 & $5675(12)$ & $6630.5(17)$ & $6674(5)$ & $74.2(16)$ \\
O3 & $7343(9)$ & $5856(2)$ & $9867(5)$ & $76.0(17)$ \\
N1 & $5947.8(19)$ & $5296(5)$ & $55.4(14)$ \\
C1 & $6569(11)$ & $6127(2)$ & $5002(7)$ & $64(2)$ \\
C2 & $3481(12)$ & $6442(3)$ & $3602(7)$ & $66(2)$ \\
C3 & $1332(15)$ & $6618(3)$ & $2654(7)$ & $64.0(19)$ \\
C4 & $1098(16)$ & $6203(3)$ & $3051(7)$ & $66(2)$ \\
C5 & $962(15)$ & $5874(3)$ & $4468(7)$ & $61.7(19)$ \\
C6 & $5712(3)$ & $7891(7)$ & $50.1(15)$ \\
C7 & $5901(2)$ & $7990(7)$ & $53.3(16)$ \\
C8 & $5370(2)$ & $7120(8)$ & $64(2)$ \\
C9 & $3341(16)$ & $8526(8)$ & $65(2)$ \\
C10 & $3545(13)$ & $5079(3)$ & $9229(7)$ & $56.8(17)$ \\
C11 & $3716(14)$ & $5117(3)$ & $9633(7)$ & $58.4(18)$ \\
C12 & $1782(15)$ & $6128(2)$ & $9067(7)$ & $60.2(18)$ \\
C13 & $1730(15)$ & $6582(2)$ & $9494(8)$ & $76(2)$ \\
C14 & $4715(14)$ & $6751(3)$ & $10498(9)$ & $81(3)$ \\
C15 & $3667(15)$ & $7169(3)$ & $11060(9)$ & $82(3)$ \\
C16 & $1327(15)$ & $7415(3)$ & $10657(7)$ & $69(2)$
\end{tabular}


Table S16. Anisotropic Displacement Parameters $\left(\AA^{2} \times 10^{3}\right)$ for 54. The Anisotropic displacement factor exponent takes the form: $-2 \pi^{2}\left[h^{2} a^{* 2} U_{11}+2 h k a * b * U_{12}+\ldots\right]$.

\begin{tabular}{lrrrrrr} 
Atom & \multicolumn{1}{c}{$\mathbf{U}_{\mathbf{1 1}}$} & \multicolumn{1}{c}{$\mathbf{U}_{\mathbf{2 2}}$} & \multicolumn{1}{c}{$\mathbf{U}_{\mathbf{3 3}}$} & \multicolumn{1}{c}{$\mathbf{U}_{\mathbf{2 3}}$} & \multicolumn{1}{c}{$\mathbf{U}_{\mathbf{1 2}}$} \\
$\mathrm{S} 1$ & $65.5(11)$ & $57.5(10)$ & $49.6(10)$ & $6.2(8)$ & $8.4(8)$ & $-8.7(9)$ \\
$\mathrm{O} 1$ & $122(5)$ & $45(3)$ & $52(3)$ & $5(2)$ & $7(3)$ & $-29(3)$ \\
$\mathrm{O} 2$ & $46(3)$ & $111(5)$ & $66(3)$ & $5(3)$ & $11(2)$ & $1(3)$ \\
$\mathrm{O} 3$ & $97(4)$ & $55(3)$ & $63(3)$ & $-2(3)$ & $-20(3)$ & $10(3)$ \\
$\mathrm{N} 1$ & $75(4)$ & $48(3)$ & $42(3)$ & $-1(2)$ & $7(3)$ & $4(3)$ \\
$\mathrm{C} 1$ & $75(5)$ & $67(5)$ & $48(4)$ & $-1(3)$ & $6(4)$ & $13(4)$ \\
$\mathrm{C} 2$ & $74(5)$ & $69(5)$ & $52(4)$ & $8(4)$ & $3(4)$ & $12(4)$ \\
$\mathrm{C} 3$ & $70(5)$ & $70(5)$ & $49(4)$ & $-1(4)$ & $2(3)$ & $-5(4)$ \\
$\mathrm{C} 4$ & $82(5)$ & $62(5)$ & $55(4)$ & $-13(4)$ & $11(4)$ & $-1(4)$ \\
$\mathrm{C} 5$ & $77(5)$ & $49(4)$ & $56(4)$ & $3(3)$ & $3(4)$ & $2(3)$ \\
$\mathrm{C} 6$ & $53(4)$ & $38(3)$ & $59(4)$ & $1(3)$ & $10(3)$ & $-1(3)$ \\
$\mathrm{C} 7$ & $59(4)$ & $41(3)$ & $59(4)$ & $2(3)$ & $10(3)$ & $0(3)$ \\
$\mathrm{C} 8$ & $74(5)$ & $46(4)$ & $71(5)$ & $0(3)$ & $7(4)$ & $-7(3)$ \\
$\mathrm{C} 9$ & $76(5)$ & $48(4)$ & $71(5)$ & $14(4)$ & $14(4)$ & $1(4)$ \\
C10 & $73(5)$ & $47(4)$ & $47(4)$ & $4(3)$ & $1(3)$ & $-1(3)$ \\
C11 & $82(5)$ & $46(4)$ & $47(4)$ & $-2(3)$ & $11(4)$ & $-6(3)$ \\
C12 & $80(5)$ & $47(4)$ & $53(4)$ & $2(3)$ & $9(4)$ & $7(4)$ \\
C13 & $106(7)$ & $65(5)$ & $60(5)$ & $3(4)$ & $21(5)$ & $19(5)$ \\
C14 & $121(8)$ & $52(5)$ & $79(6)$ & $-13(4)$ & $46(6)$ & $-5(5)$ \\
C15 & $126(9)$ & $60(5)$ & $66(5)$ & $-12(4)$ & $34(6)$ & $-18(5)$ \\
C16 & $91(6)$ & $66(5)$ & $52(4)$ & $0(4)$ & $15(4)$ & $-11(4)$
\end{tabular}

Table S17. Bond Lengths for 54.

\begin{tabular}{llrllr}
\multicolumn{2}{c}{ Atom Atom } & Length/ $\boldsymbol{\AA}$ & \multicolumn{1}{c}{ Atom } & \multicolumn{1}{c}{ Atom } & \multicolumn{1}{c}{ Length/ $\boldsymbol{\AA}$} \\
S1 & O1 & $1.433(5)$ & C6 & C10 & $1.563(10)$ \\
S1 & O2 & $1.418(6)$ & C7 & C8 & $1.506(10)$ \\
S1 & N1 & $1.616(6)$ & C7 & C 9 & $1.488(10)$ \\
S1 & C6 & $1.786(7)$ & C8 & C9 & $1.472(11)$ \\
O3 & C10 & $1.217(9)$ & C10 & C11 & $1.493(10)$ \\
N1 & C1 & $1.459(9)$ & C11 & C12 & $1.391(11)$ \\
N1 & C5 & $1.450(9)$ & C11 & C16 & $1.394(10)$ \\
C1 & C2 & $1.524(10)$ & C12 & C13 & $1.380(10)$ \\
C2 & C3 & $1.525(11)$ & C13 & C14 & $1.375(13)$ \\
C3 & C4 & $1.505(11)$ & C14 & C15 & $1.364(14)$ \\
C4 & C5 & $1.529(10)$ & C15 & C16 & $1.386(12)$ \\
C6 & C7 & $1.503(9)$ & & &
\end{tabular}


Table S18. Bond Angles for 54.

\begin{tabular}{|c|c|c|c|c|c|c|c|}
\hline \multicolumn{2}{|c|}{ Atom Atom } & \multirow[t]{2}{*}{ Atom } & \multirow{2}{*}{$\begin{array}{l}\text { Angle/ }{ }^{\circ} \\
108.0(3)\end{array}$} & Atom & \multirow[t]{2}{*}{ Atom } & \multirow{2}{*}{$\begin{array}{l}\text { Atom } \\
\text { C8 }\end{array}$} & \multirow{2}{*}{$\begin{array}{l}\text { Angle/ }{ }^{\circ} \\
\qquad 118.4(6)\end{array}$} \\
\hline $\mathrm{O} 1$ & $\mathrm{~S} 1$ & & & C6 & & & \\
\hline $\mathrm{O} 1$ & S1 & C6 & $106.2(3)$ & C9 & $\mathrm{C} 7$ & C6 & $117.7(6)$ \\
\hline $\mathrm{O} 2$ & S1 & $\mathrm{O} 1$ & $117.3(4)$ & C9 & $\mathrm{C} 7$ & $\mathrm{C} 8$ & $58.9(5)$ \\
\hline $\mathrm{O} 2$ & S1 & N1 & $107.4(3)$ & C9 & $\mathrm{C} 8$ & $\mathrm{C} 7$ & $59.9(5)$ \\
\hline $\mathrm{O} 2$ & $\mathrm{~S} 1$ & C6 & $109.0(3)$ & $\mathrm{C} 8$ & C9 & $\mathrm{C} 7$ & $61.2(5)$ \\
\hline N1 & $\mathrm{S} 1$ & C6 & $108.9(3)$ & $\mathrm{O} 3$ & $\mathrm{C} 10$ & C6 & $118.7(7)$ \\
\hline $\mathrm{C} 1$ & N1 & $\mathrm{S} 1$ & $119.6(5)$ & $\mathrm{O} 3$ & $\mathrm{C} 10$ & $\mathrm{C} 11$ & $121.9(7)$ \\
\hline $\mathrm{C} 5$ & N1 & $\mathrm{S} 1$ & $122.0(5)$ & C11 & $\mathrm{C} 10$ & C6 & $119.3(6)$ \\
\hline C5 & N1 & $\mathrm{C} 1$ & $114.4(6)$ & $\mathrm{C} 12$ & C11 & $\mathrm{C} 10$ & $123.0(7)$ \\
\hline N1 & $\mathrm{C} 1$ & $\mathrm{C} 2$ & $109.0(6)$ & $\mathrm{C} 12$ & C11 & $\mathrm{C} 16$ & $118.9(7)$ \\
\hline C3 & $\mathrm{C} 2$ & $\mathrm{C} 1$ & $110.7(6)$ & $\mathrm{C} 16$ & C11 & $\mathrm{C} 10$ & $118.1(7)$ \\
\hline $\mathrm{C} 4$ & C3 & $\mathrm{C} 2$ & $110.6(6)$ & C13 & $\mathrm{C} 12$ & C11 & $120.5(8)$ \\
\hline C3 & $\mathrm{C} 4$ & $\mathrm{C} 5$ & $111.6(7)$ & $\mathrm{C} 12$ & C13 & $\mathrm{C} 14$ & $119.4(9)$ \\
\hline N1 & $\mathrm{C} 5$ & $\mathrm{C} 4$ & $108.6(6)$ & $\mathrm{C} 15$ & $\mathrm{C} 14$ & C13 & $121.4(8)$ \\
\hline $\mathrm{C} 7$ & C6 & $\mathrm{S} 1$ & $113.4(5)$ & $\mathrm{C} 14$ & $\mathrm{C} 15$ & $\mathrm{C} 16$ & $119.6(9)$ \\
\hline $\mathrm{C} 7$ & C6 & $\mathrm{C} 10$ & $109.8(6)$ & $\mathrm{C} 15$ & $\mathrm{C} 16$ & $\mathrm{C} 11$ & $120.2(9)$ \\
\hline C10 & C6 & S1 & $105.7(5)$ & & & & \\
\hline
\end{tabular}

Table S19. Hydrogen Atom Coordinates $\left(\AA \times 10^{4}\right)$ and Isotropic Displacement Parameters $\left(\AA^{2} \times 10^{3}\right)$ for 54 .

$\begin{array}{lrrrr}\text { Atom } & \boldsymbol{x} & \boldsymbol{y} & \boldsymbol{z} & \mathbf{U}(\mathbf{e q}) \\ \text { H1A } & 1542.3 & 6708.06 & 5599.96 & 77 \\ \text { H1B } & -170.52 & 6273.14 & 5107.13 & 77 \\ \text { H2A } & -392.39 & 6809.63 & 3378.86 & 79 \\ \text { H2B } & 2524.96 & 6813.96 & 3525.87 & 79 \\ \text { H3A } & -583.33 & 6031.1 & 2641.32 & 77 \\ \text { H3B } & 971.5 & 6320.99 & 1779.65 & 77 \\ \text { H4A } & 4662.61 & 6033.64 & 2947.51 & 80 \\ \text { H4B } & 2938.38 & 5599.04 & 2482.06 & 80 \\ \text { H5A } & 1897.09 & 5523.14 & 4562.14 & 74 \\ \text { H5B } & 4821.21 & 5518.91 & 4719.28 & 74 \\ \text { H6 } & 5993.9 & 7662.15 & 60 \\ \text { H7 } & 1789.39 & 5235.75 & 8196.78 & 64 \\ \text { H8A } & 5397.17 & 5245.11 & 6519.03 & 77 \\ \text { H8B } & 505.51 & 4779.66 & 6798.91 & 77 \\ \text { H9A } & 2305.93 & 4840.46 & 9065.26 & 78 \\ \text { H9B } & 2212.81 & 5305.51 & 8785.61 & 78 \\ \text { H12 } & 413.91 & 6581.96 & 8395.52 & 72 \\ \text { H13 } & 355.56 & 7283.65 & 9107.88 & 92 \\ \text { H14 } & -1122.52 & 7692.33 & 10798.78 & 97\end{array}$




\section{Experimental}

Single crystals of $\mathrm{C}_{16} \mathrm{H}_{21} \mathrm{NO}_{3} \mathrm{~S}$ (54) were grown via slow diffusion of pentane through a solution of 54 in acetone. A suitable crystal was selected and analyzed on a Bruker Venture Photon 2 Microsource diffractometer. The crystal was kept at $110 \mathrm{~K}$ during data collection. Using Olex2 [1], the structure was solved with the ShelXT [2] structure solution program using Intrinsic Phasing and refined with the ShelXL [3] refinement package using Least Squares minimization.

1. Dolomanov, O.V., Bourhis, L.J., Gildea, R.J, Howard, J.A.K. \& Puschmann, H. (2009), J. Appl. Cryst. 42, 339-341.

2. Sheldrick, G.M. (2015). Acta Cryst. A71, 3-8.

3. Sheldrick, G.M. (2015). Acta Cryst. C71, 3-8.

\section{Crystal structure determination of 54}

Crystal Data for $\mathrm{C}_{16} \mathrm{H}_{21} \mathrm{NO}_{3} \mathrm{~S}(M=307.40 \mathrm{~g} / \mathrm{mol})$ : monoclinic, space group $\mathrm{P} 2{ }_{1} / \mathrm{n}$ (no. 14), $a=5.4513(3) \AA, b=28.2495(18) \AA, c=10.4075(8) \AA, \beta=100.332(7)^{\circ}, V=1576.73(18) \AA^{3}, Z=$ $4, T=110 \mathrm{~K}, \mu(\mathrm{CuK} \alpha)=1.904 \mathrm{~mm}^{-1}$, Dcalc $=1.295 \mathrm{~g} / \mathrm{cm}^{3}, 36657$ reflections measured $\left(6.258^{\circ} \leq\right.$ $\left.2 \Theta \leq 158.368^{\circ}\right), 3355$ unique $\left(R_{\text {int }}=0.1305, \mathrm{R}_{\text {sigma }}=0.0510\right)$ which were used in all calculations. The final $R_{1}$ was $0.1395(\mathrm{I}>2 \sigma(\mathrm{I}))$ and $w R_{2}$ was 0.3043 (all data).

\section{Refinement model description}

Number of restraints - 0 , number of constraints - unknown.

Details:

1. Fixed Uiso

At 1.2 times of:

All $\mathrm{C}(\mathrm{H})$ groups, All $\mathrm{C}(\mathrm{H}, \mathrm{H})$ groups

2.a Ternary $\mathrm{CH}$ refined with riding coordinates:

C6(H6), C7(H7)

2.b Secondary $\mathrm{CH} 2$ refined with riding coordinates:

C1(H1A,H1B), C2(H2A,H2B), C3(H3A,H3B), C4(H4A,H4B), C5(H5A,H5B), C8(H8A,H8B), C9(H9A,H9B)

2.c Aromatic/amide $\mathrm{H}$ refined with riding coordinates:

C12(H12), C13(H13), C14(H14), C15(H15), C16(H16) 
NMR Spectra

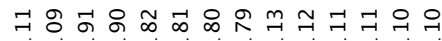

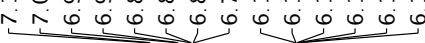

क

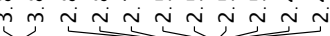<smiles>COc1ccc2c(c1)C(OS(=O)(=O)F)=CCC2</smiles>

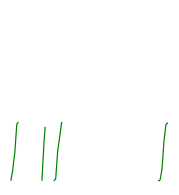

$\left({ }^{1} \mathrm{H}, 400 \mathrm{MHz}, \mathrm{CDCl}_{3}\right)$

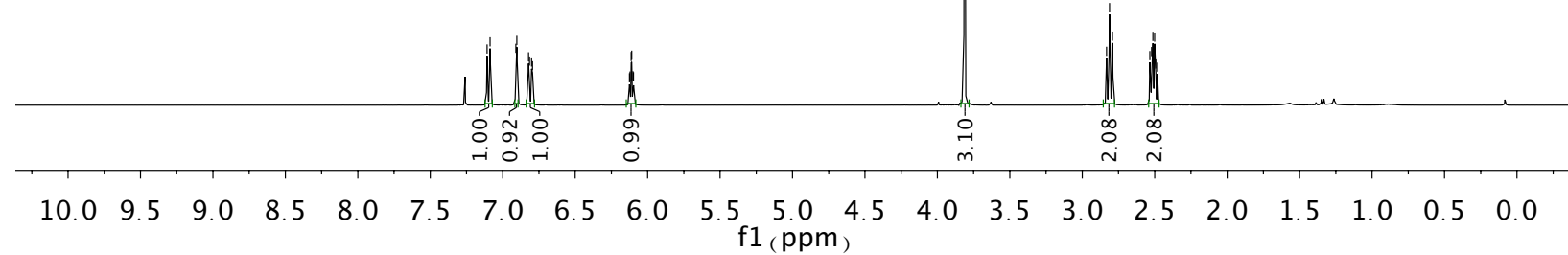

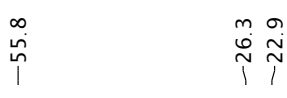

$\left({ }^{13} \mathrm{C}, 126 \mathrm{MHz}, \mathrm{CDCl}_{3}\right)$

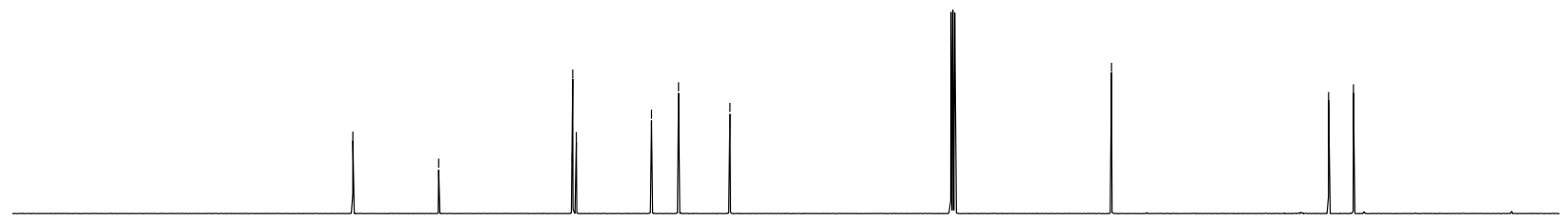

$\begin{array}{llllllllllllllllllll}200 & 190 & 180 & 170 & 160 & 150 & 140 & 130 & 120 & 110 \underset{\mathrm{f} 1(\mathrm{ppm})}{100} & 90 & 80 & 70 & 60 & 50 & 40 & 30 & 20 & 10 & 0\end{array}$ 


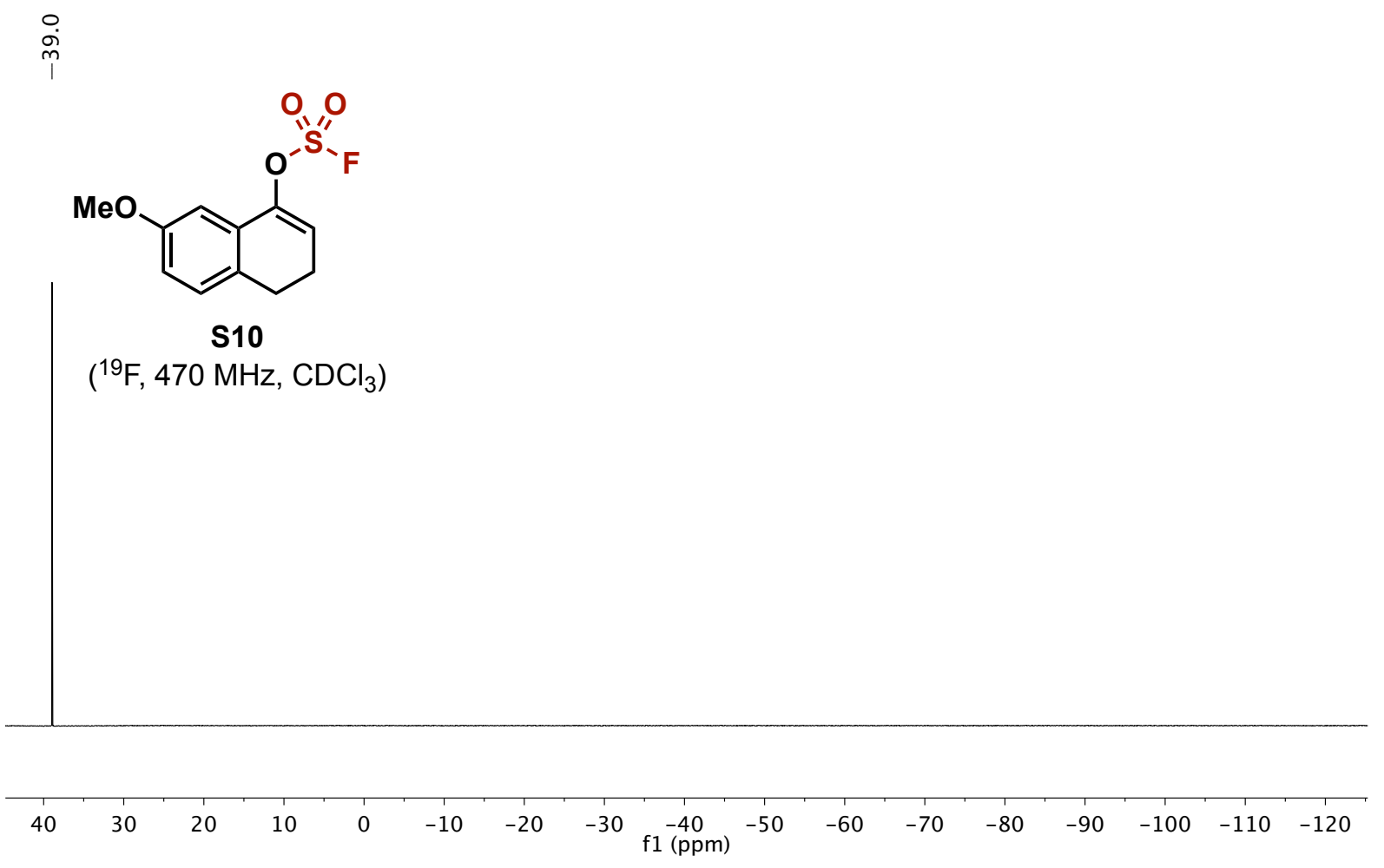




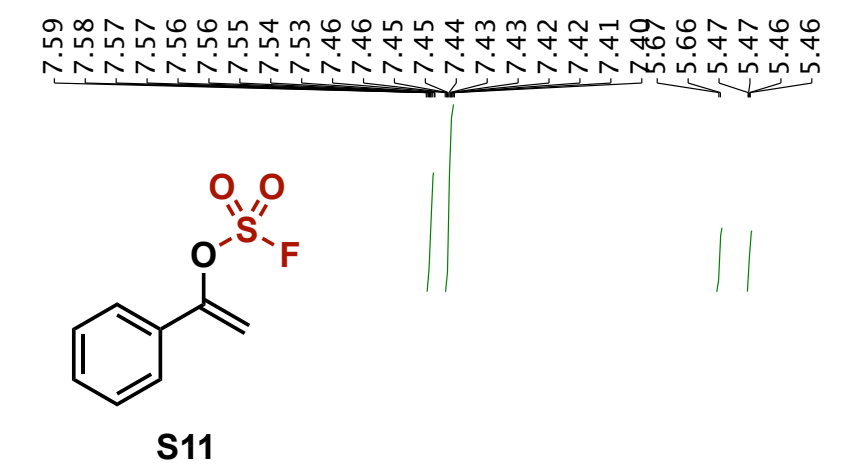

$\left({ }^{1} \mathrm{H}, 400 \mathrm{MHz}, \mathrm{CDCl}_{3}\right)$
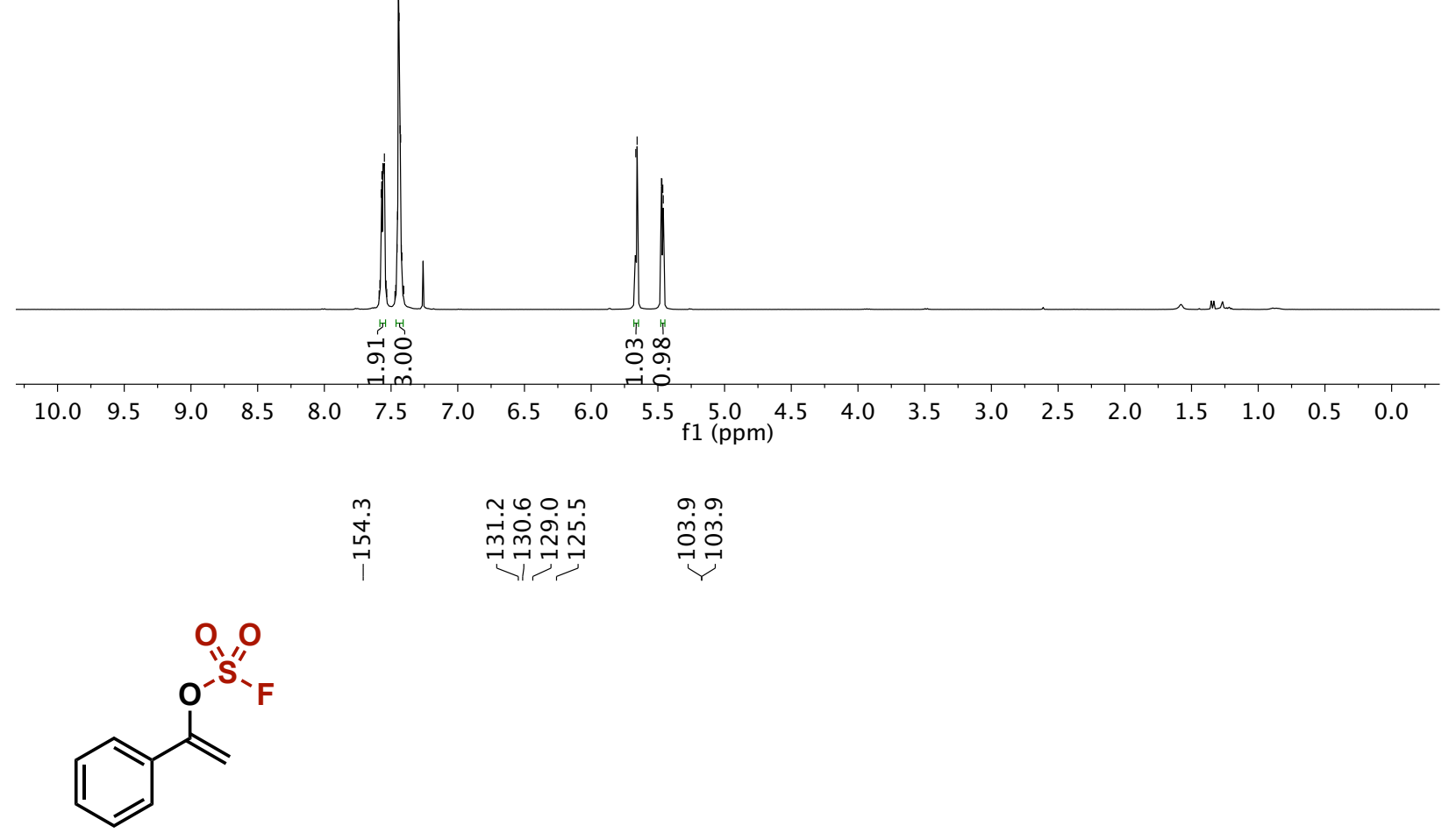

S11

$\left({ }^{13} \mathrm{C}, 126 \mathrm{MHz}, \mathrm{CDCl}_{3}\right)$

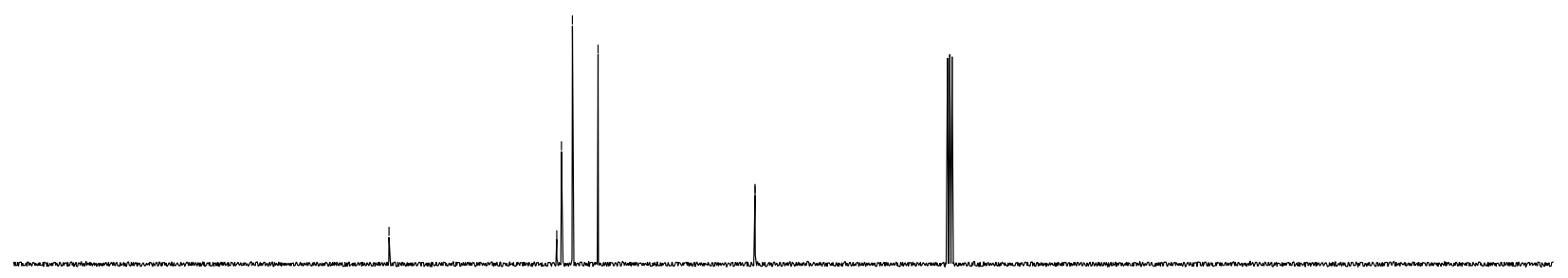

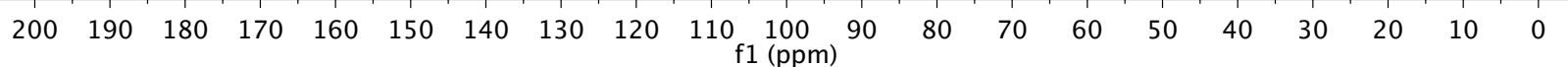




$$
\stackrel{0}{i}
$$<smiles>C=C(OS(=O)(=O)F)c1ccccc1</smiles>

$\mathrm{S} 11$

$\left({ }^{19} \mathrm{~F}, 470 \mathrm{MHz}, \mathrm{CDCl}_{3}\right)$

\begin{tabular}{|c|c|c|c|c|c|c|c|c|c|c|c|c|c|c|c|c|}
\hline 40 & 30 & 20 & 10 & 0 & -10 & -20 & -30 & f1 $\begin{array}{c}-40 \\
(\mathrm{ppm})\end{array}$ & -50 & -60 & -70 & -80 & -90 & -100 & -110 & -120 \\
\hline
\end{tabular}



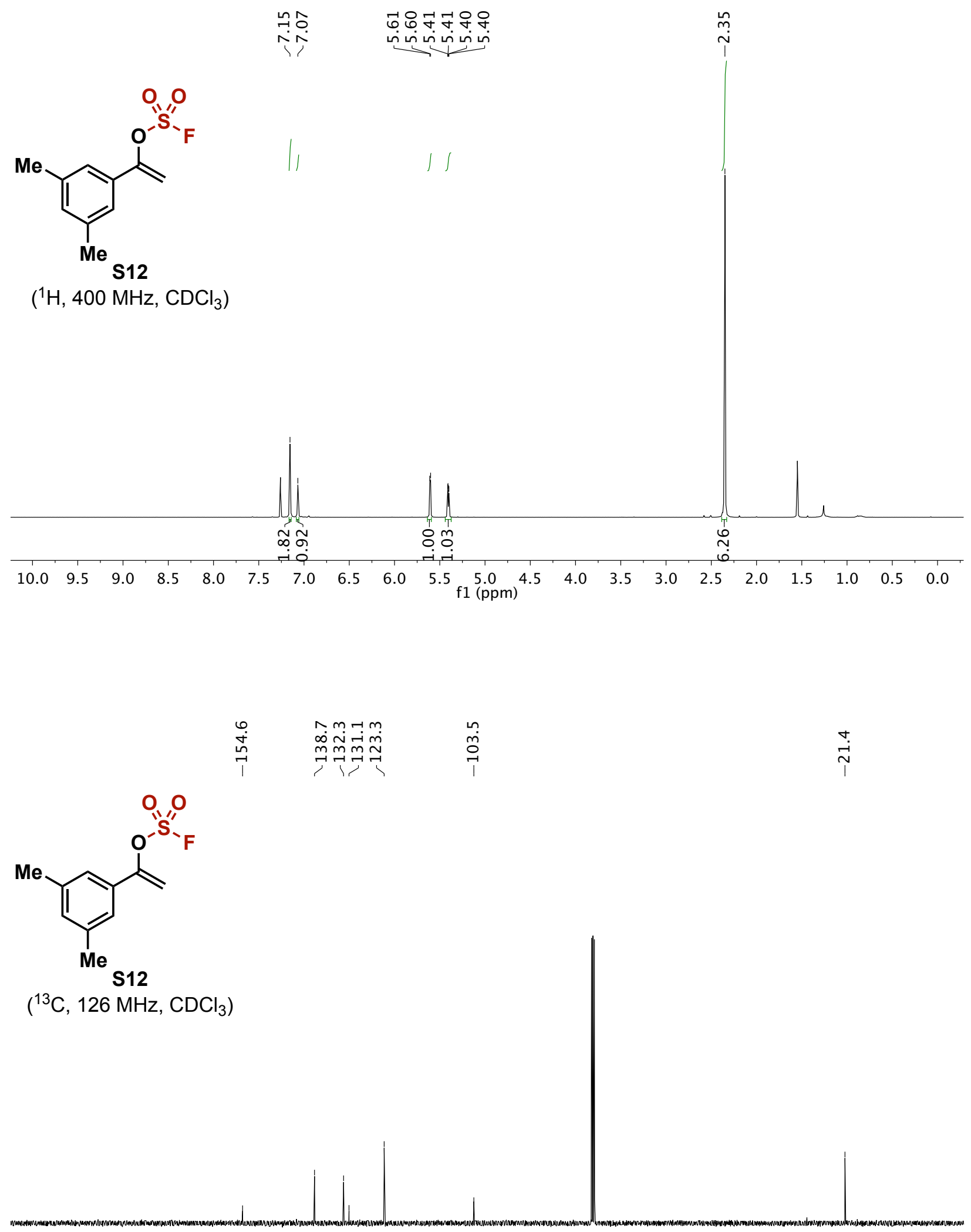

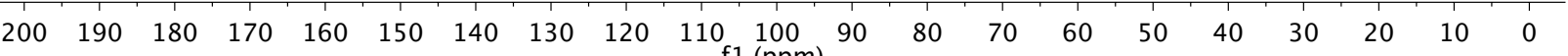




$$
\text { กั }
$$<smiles>C=C(OS(=O)(=O)F)c1cc(C)cc(C)c1</smiles>

$\left({ }^{19} \mathrm{~F}, 470 \mathrm{MHz}, \mathrm{CDCl}_{3}\right)$

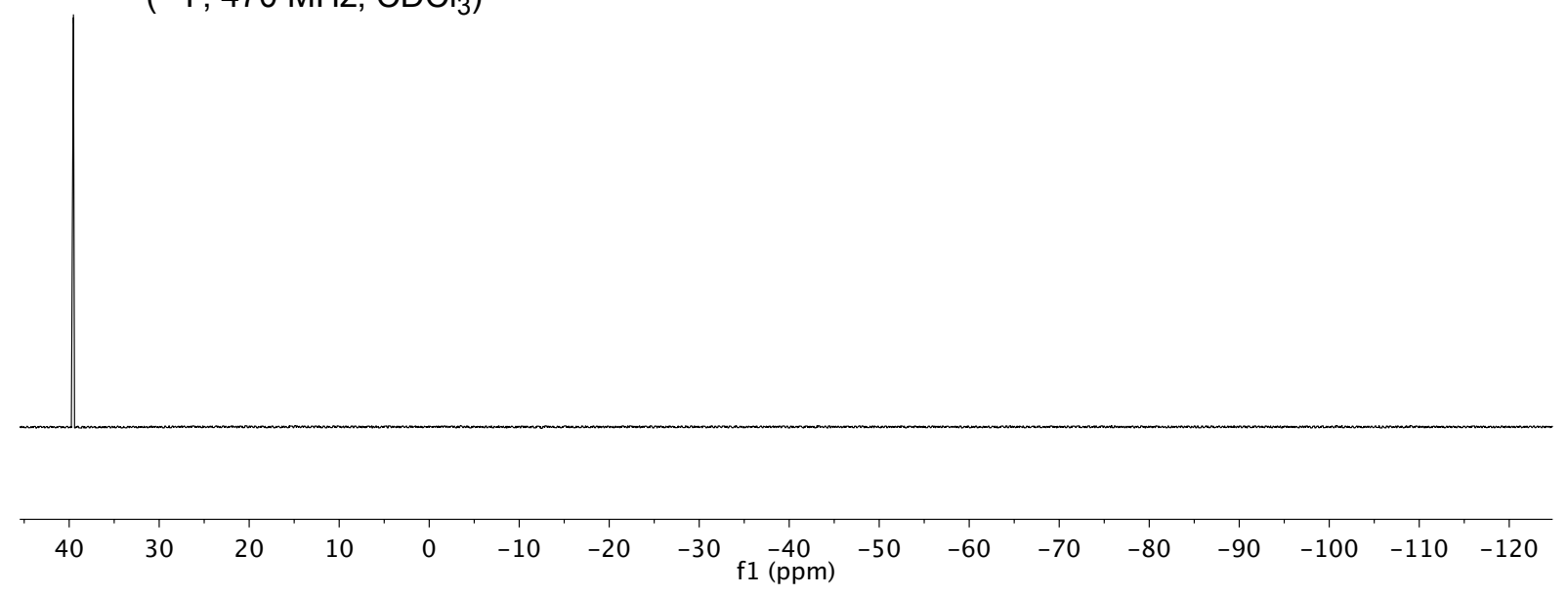



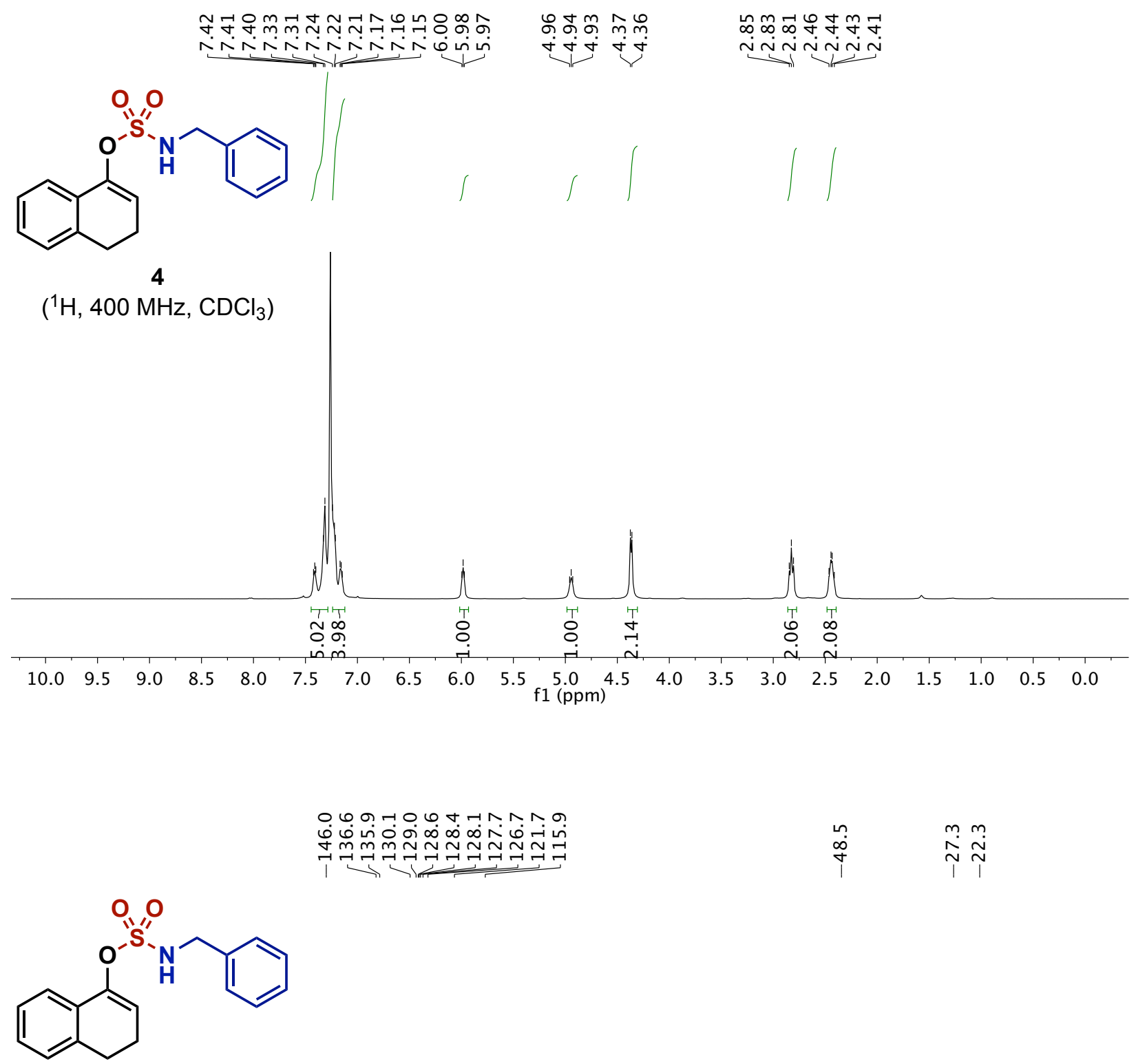

4

$\left({ }^{13} \mathrm{C}, 126 \mathrm{MHz}, \mathrm{CDCl}_{3}\right)$

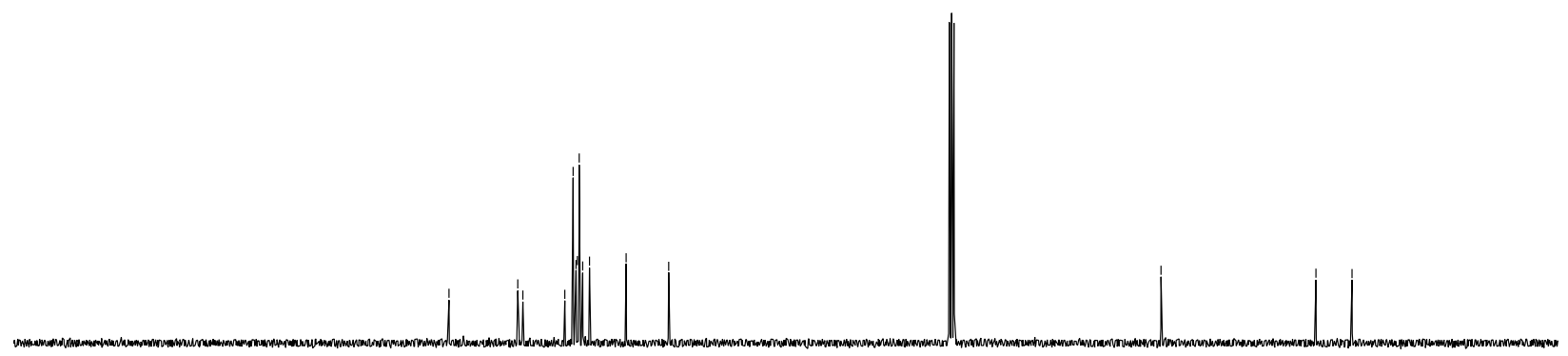

$\begin{array}{lllllllllllllllllllll}200 & 190 & 180 & 170 & 160 & 150 & 140 & 130 & 120 & 110 & 100 & 90 & 80 & 70 & 60 & 50 & 40 & 30 & 20 & 10 & 0\end{array}$ 


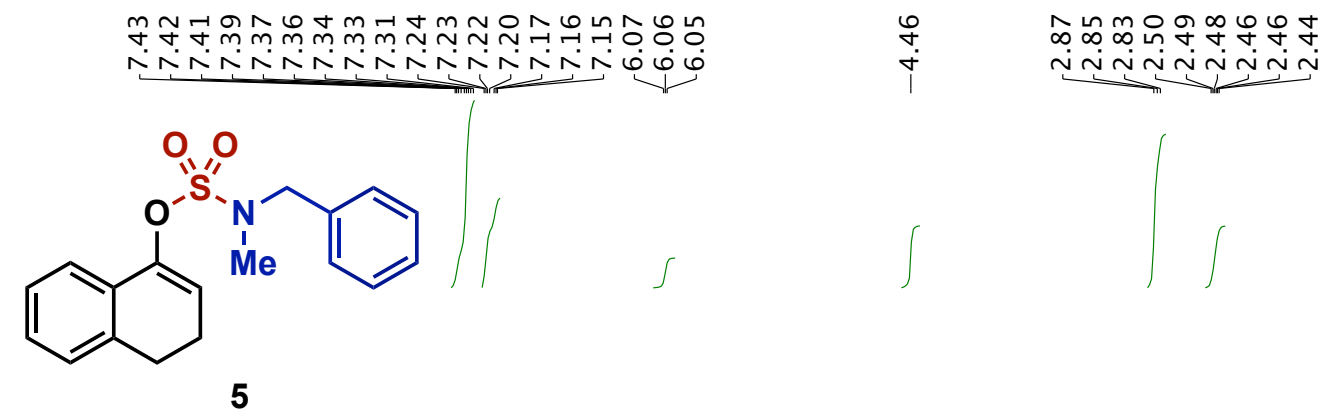

$\left({ }^{1} \mathrm{H}, 400 \mathrm{MHz}, \mathrm{CDCl}_{3}\right)$
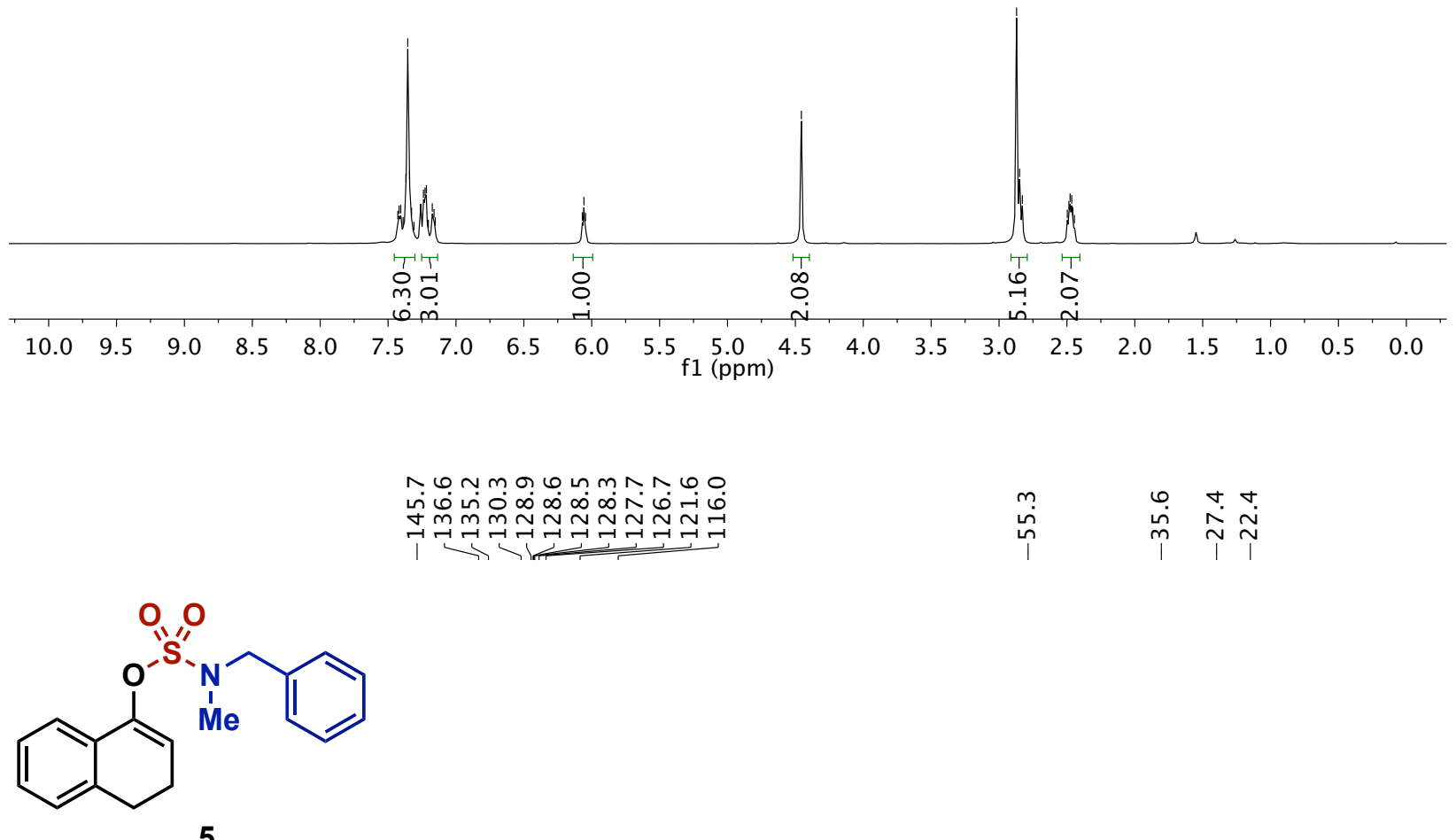

$\left({ }^{13} \mathrm{C}, 126 \mathrm{MHz}, \mathrm{CDCl}_{3}\right)$

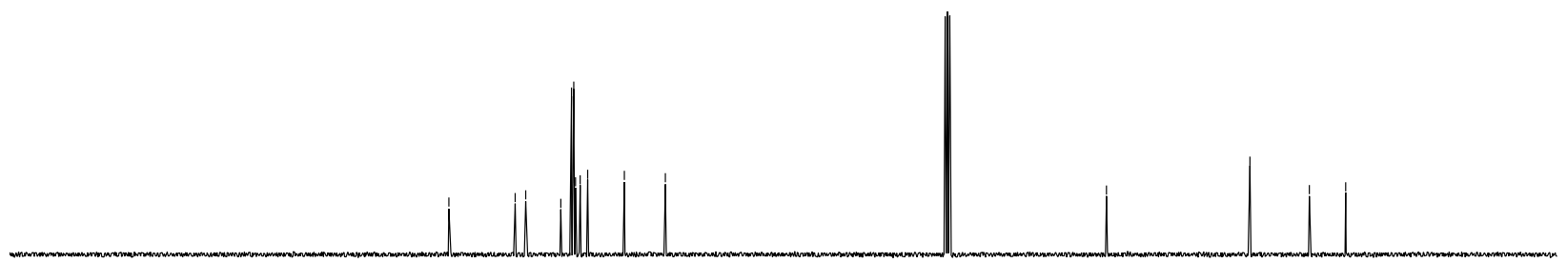

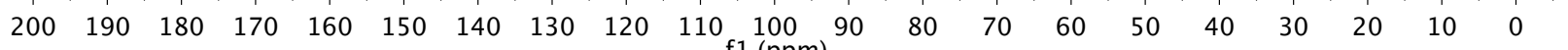




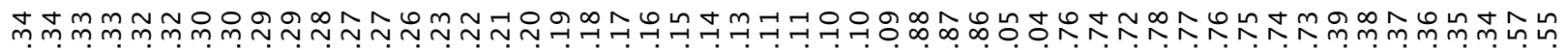

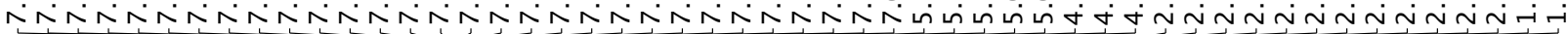<smiles>C[C@H](NS(=O)(=O)OC1=CCCc2ccccc21)c1ccccc1</smiles>

6

$\left({ }^{1} \mathrm{H}, 400 \mathrm{MHz}, \mathrm{CDCl}_{3}\right)$
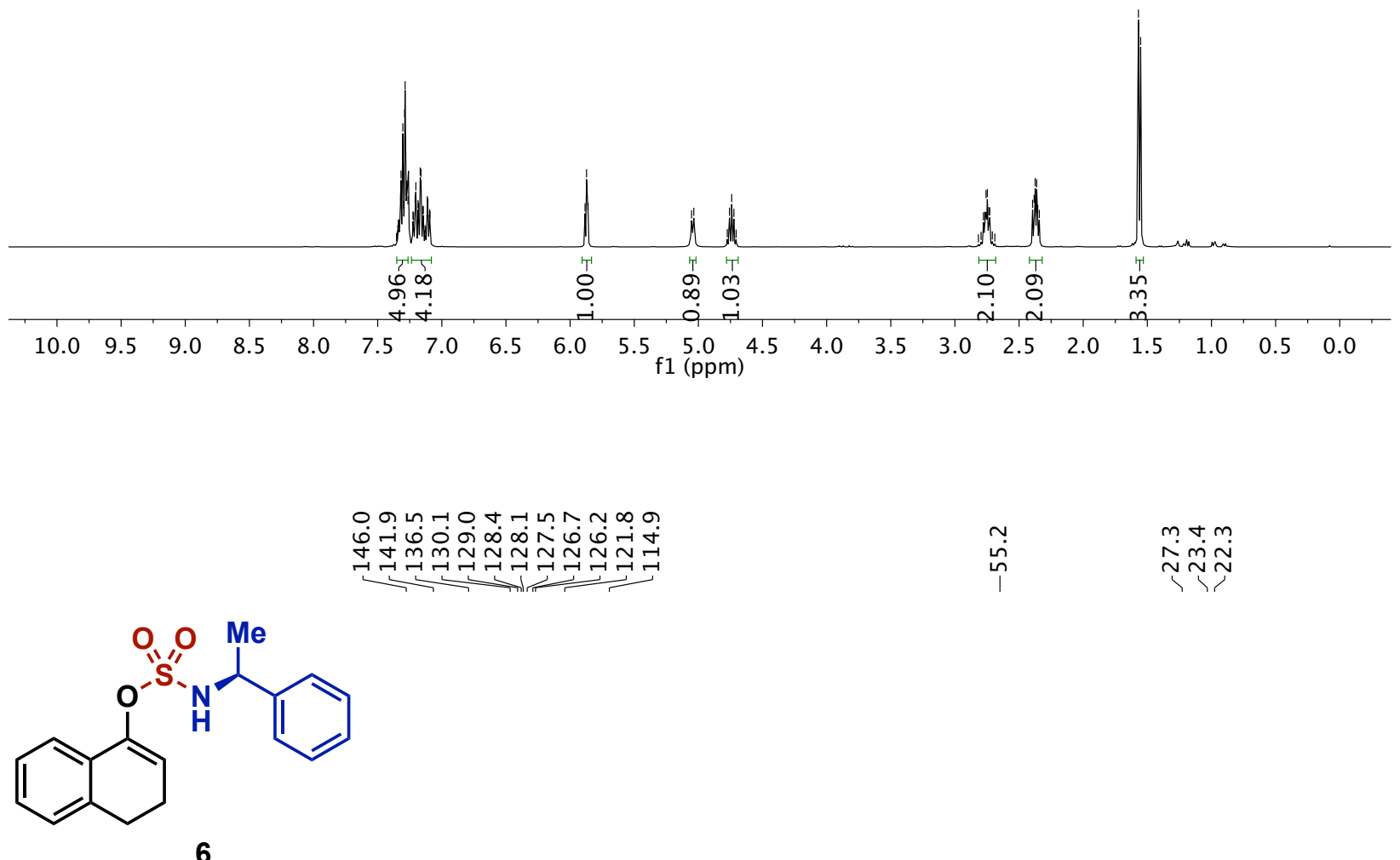

$\left({ }^{13} \mathrm{C}, 126 \mathrm{MHz}, \mathrm{CDCl}_{3}\right)$

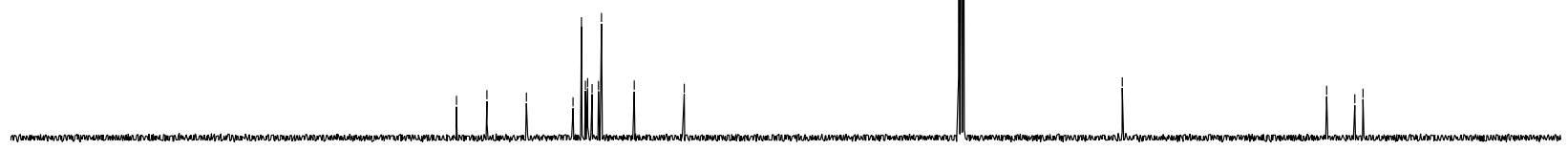

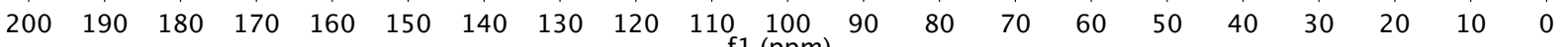




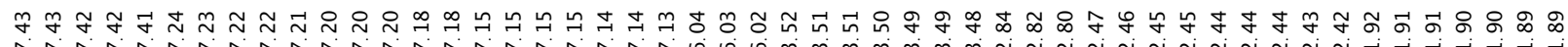

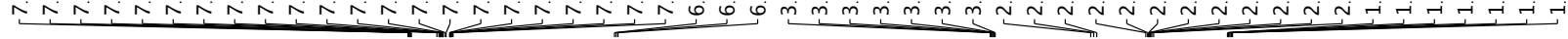<smiles>O=S(=O)(OC1=CCCc2ccccc21)N1CCCC1</smiles>

3

$\left({ }^{1} \mathrm{H}, 400 \mathrm{MHz}, \mathrm{CDCl}_{3}\right)$
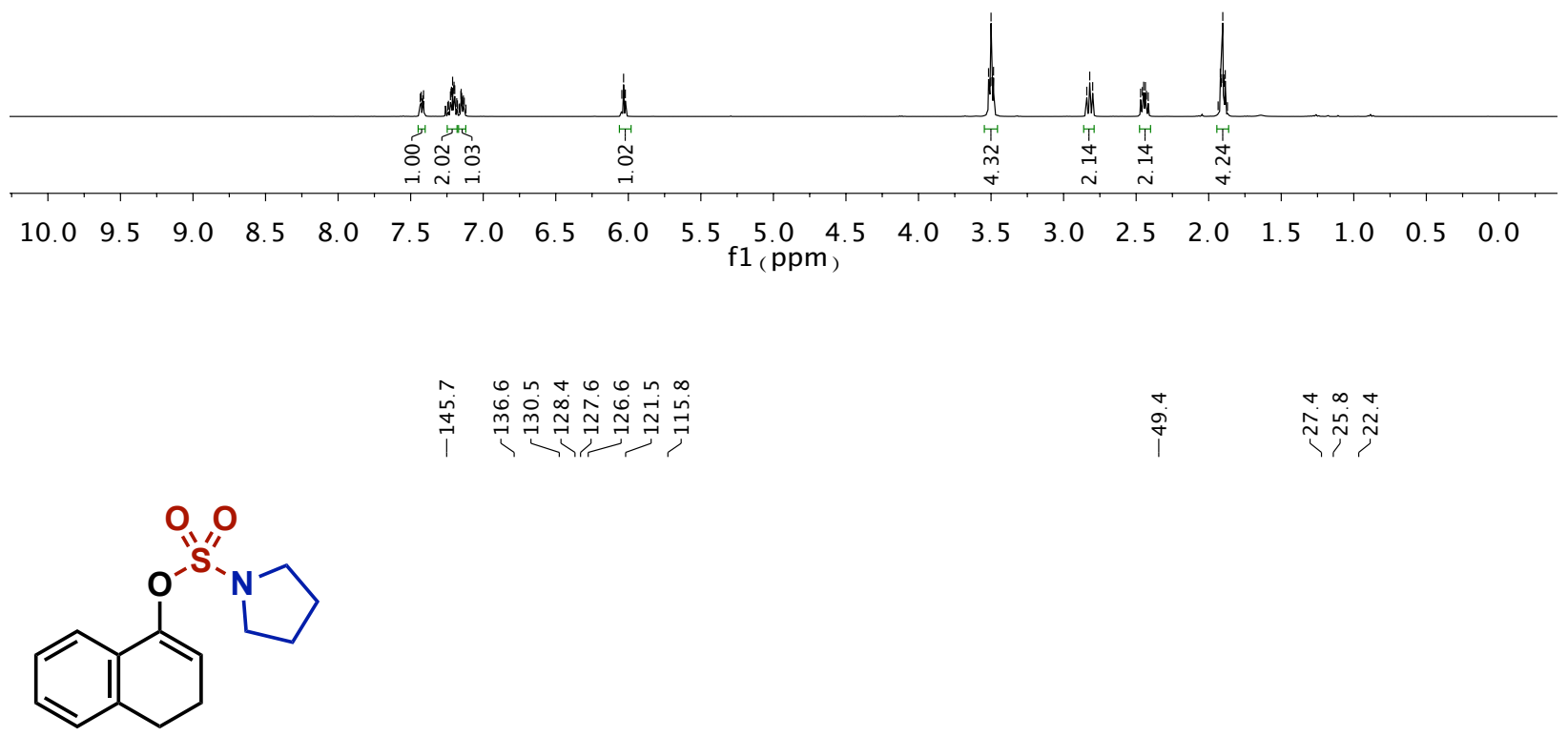

3

$\left({ }^{13} \mathrm{C}, 126 \mathrm{MHz}, \mathrm{CDCl}_{3}\right)$

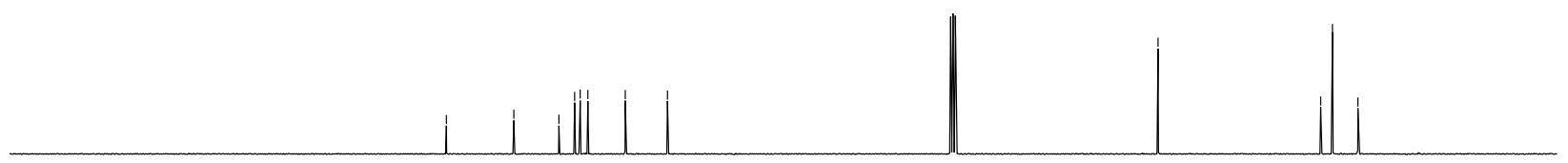

$\begin{array}{llllllllllllllllllll}200 & 190 & 180 & 170 & 160 & 150 & 140 & 130 & 120 & 110 \underset{\mathrm{f} 1}{1000}\left(\begin{array}{ll}\mathrm{ppm}) \\ 100\end{array}\right. & 80 & 70 & 60 & 50 & 40 & 30 & 20 & 10 & 0\end{array}$ 


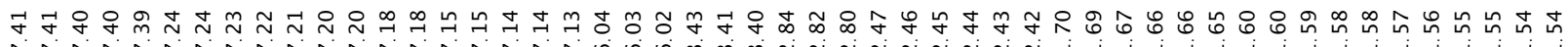

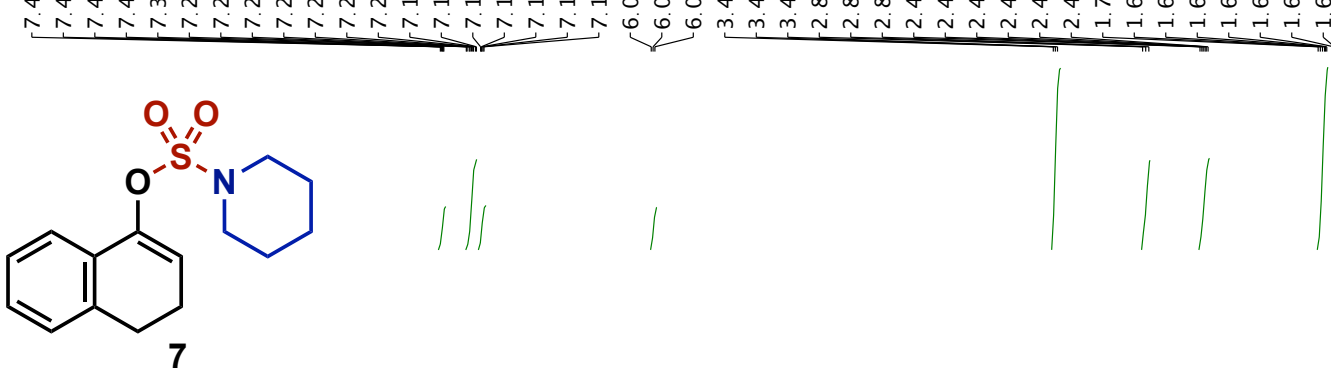

$\left({ }^{1} \mathrm{H}, 400 \mathrm{MHz}, \mathrm{CDCl}_{3}\right)$
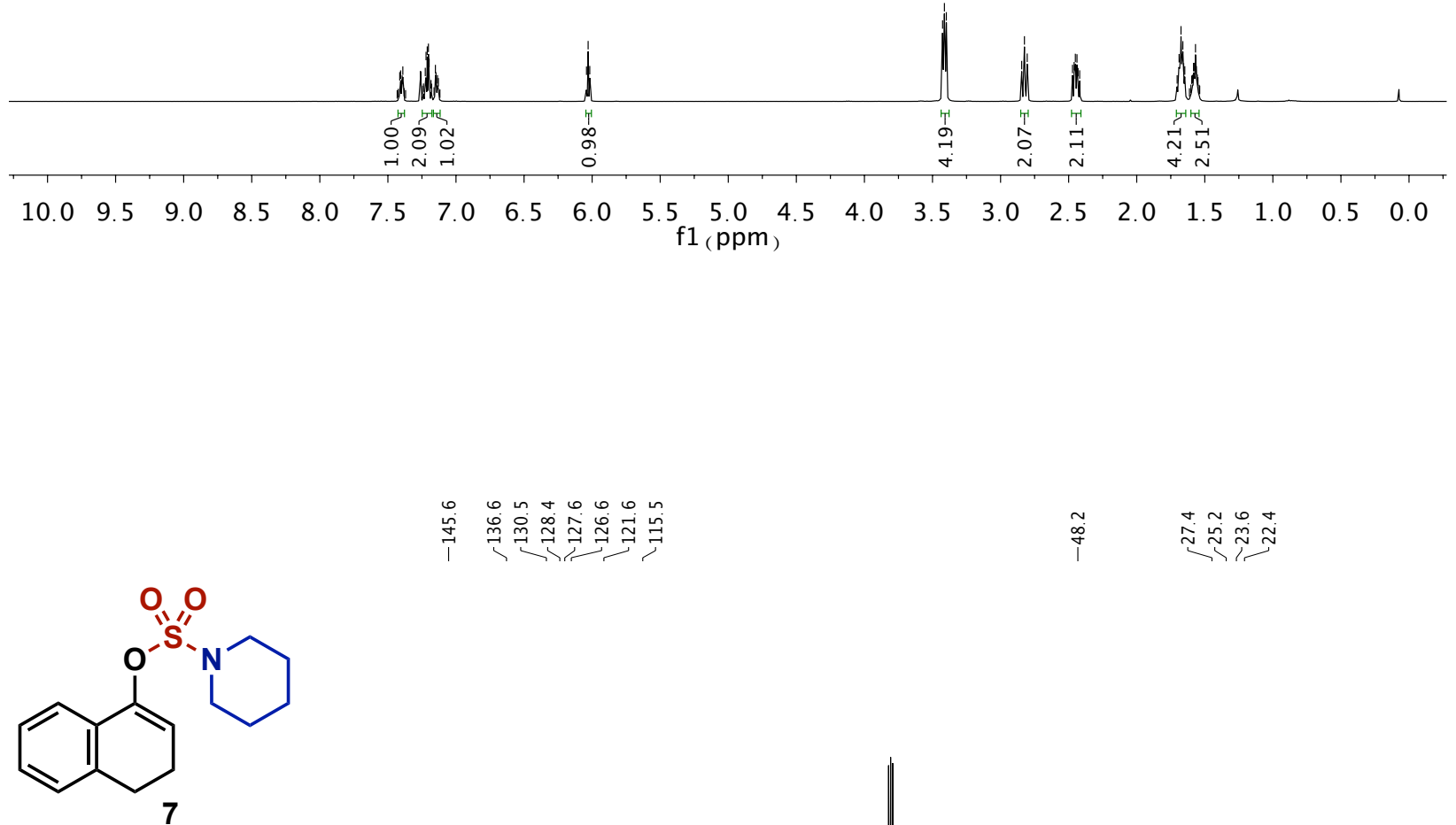

$\left({ }^{13} \mathrm{C}, 126 \mathrm{MHz}, \mathrm{CDCl}_{3}\right)$ 


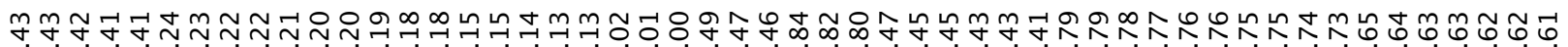

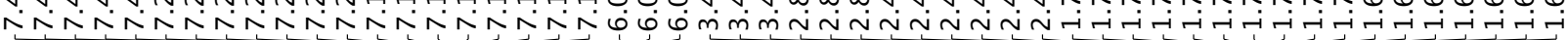<smiles>O=S(=O)(OC1=CCCc2ccccc21)N1CCCCCC1</smiles>

8

$\left({ }^{1} \mathrm{H}, 400 \mathrm{MHz}, \mathrm{CDCl}_{3}\right)$
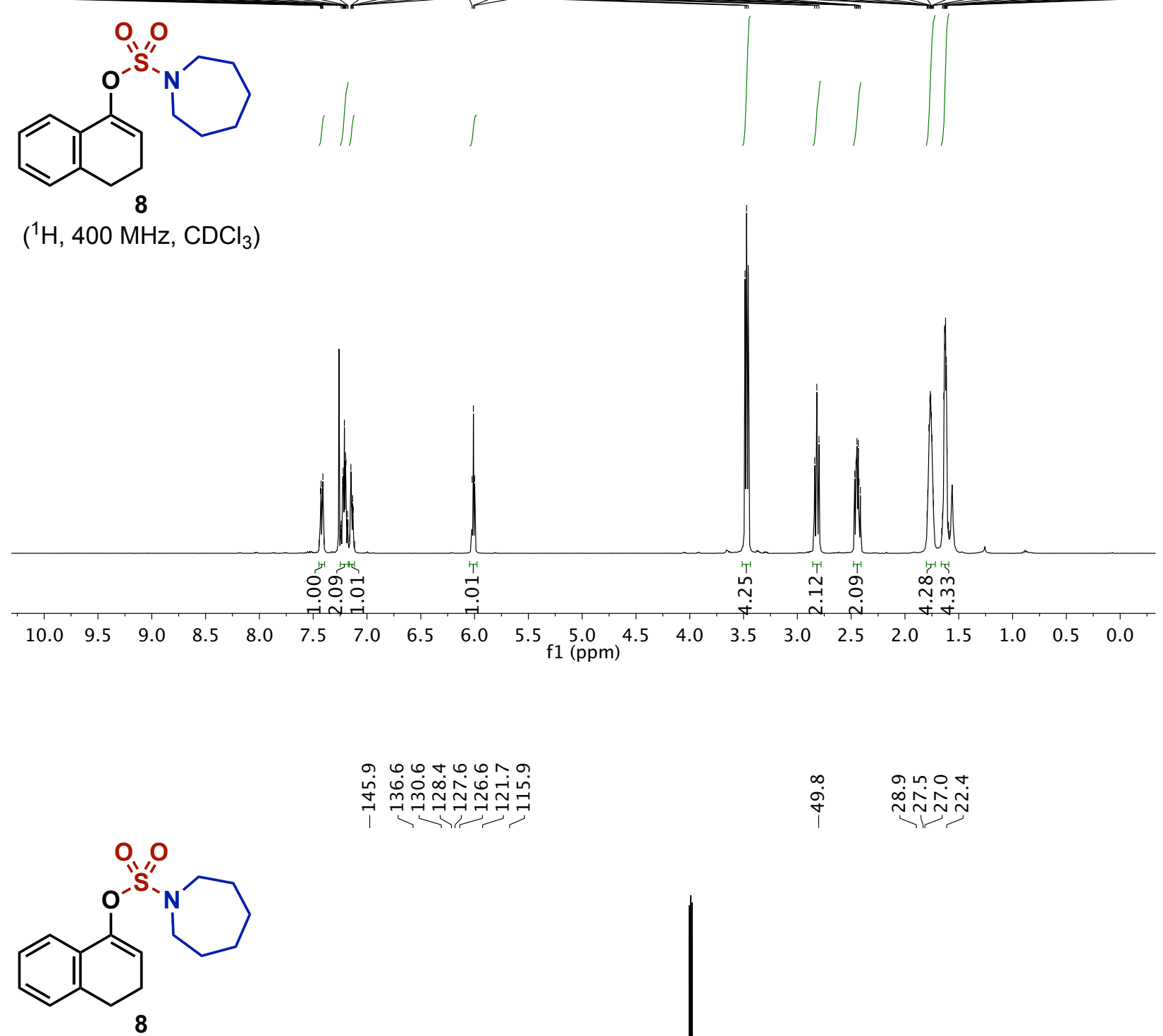

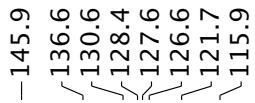

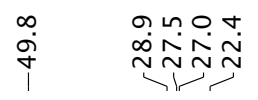

$\left({ }^{13} \mathrm{C}, 126 \mathrm{MHz}, \mathrm{CDCl}_{3}\right)$

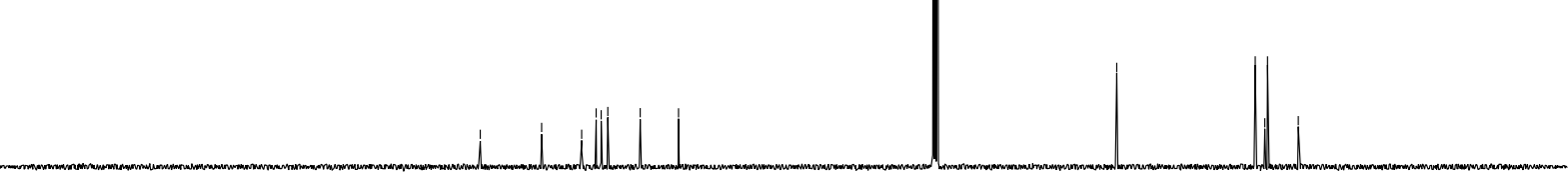

$\begin{array}{lllllllllllllllllllllll}210 & 200 & 190 & 180 & 170 & 160 & 150 & 140 & 130 & 120 & 110 \underset{\mathrm{f} 1(\mathrm{ppm})}{100} & 90 & 80 & 70 & 60 & 50 & 40 & 30 & 20 & 10 & 0 & -10\end{array}$ 


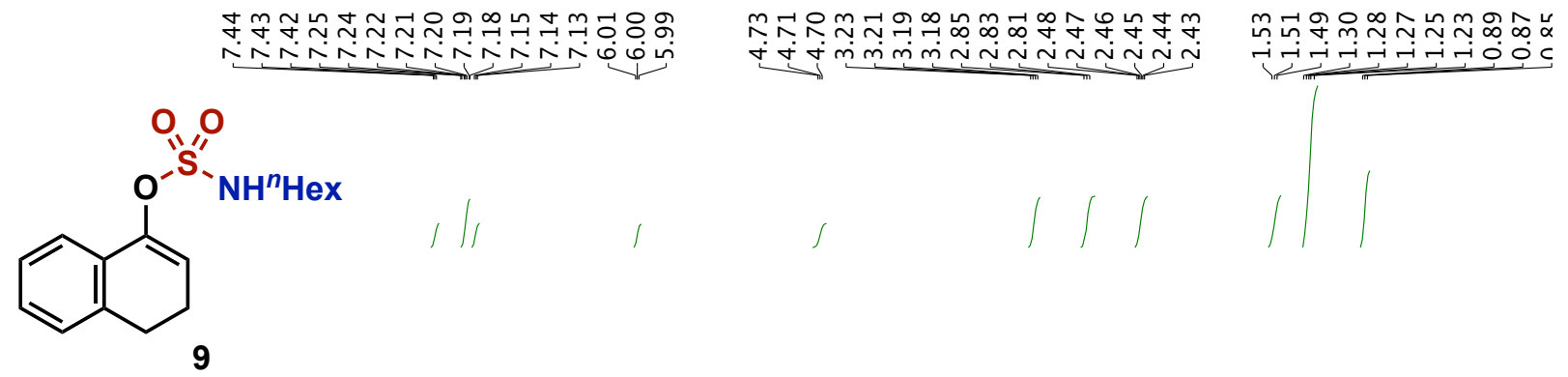

$\left({ }^{1} \mathrm{H}, 400 \mathrm{MHz}, \mathrm{CDCl}_{3}\right)$
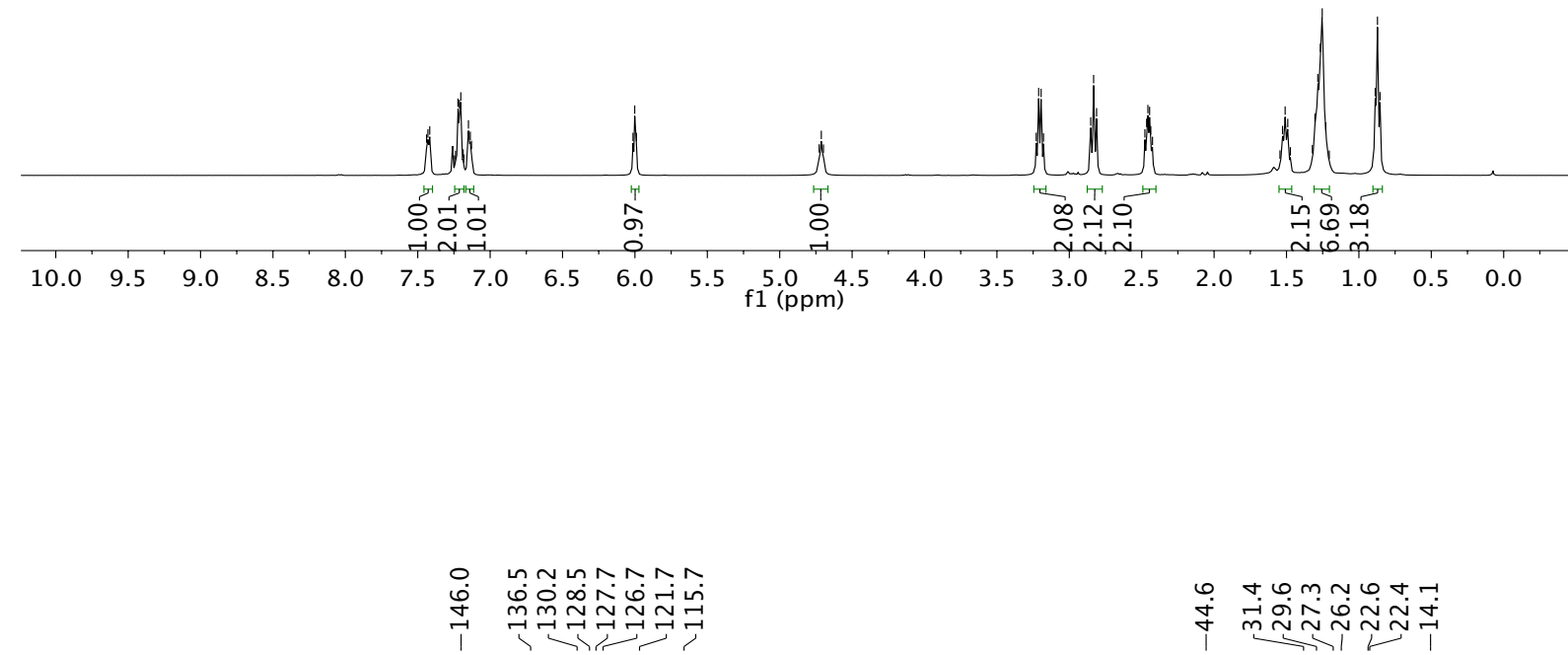

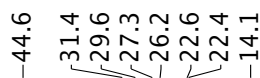

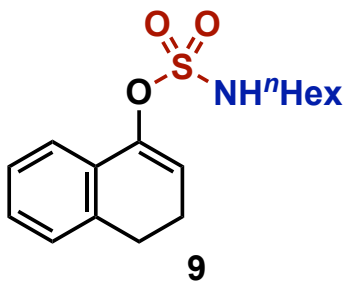

$\left({ }^{13} \mathrm{C}, 126 \mathrm{MHz}, \mathrm{CDCl}_{3}\right)$ 


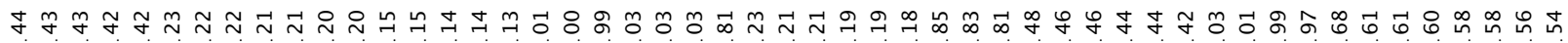

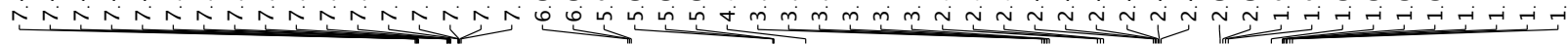<smiles>C=C(C)/C=C(\C)CCCCNS(=O)(=O)OC1=CCCc2ccccc21</smiles>

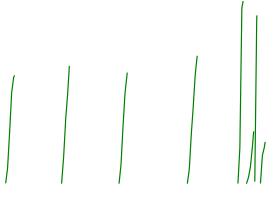

10

$\left({ }^{1} \mathrm{H}, 400 \mathrm{MHz}, \mathrm{CDCl}_{3}\right)$

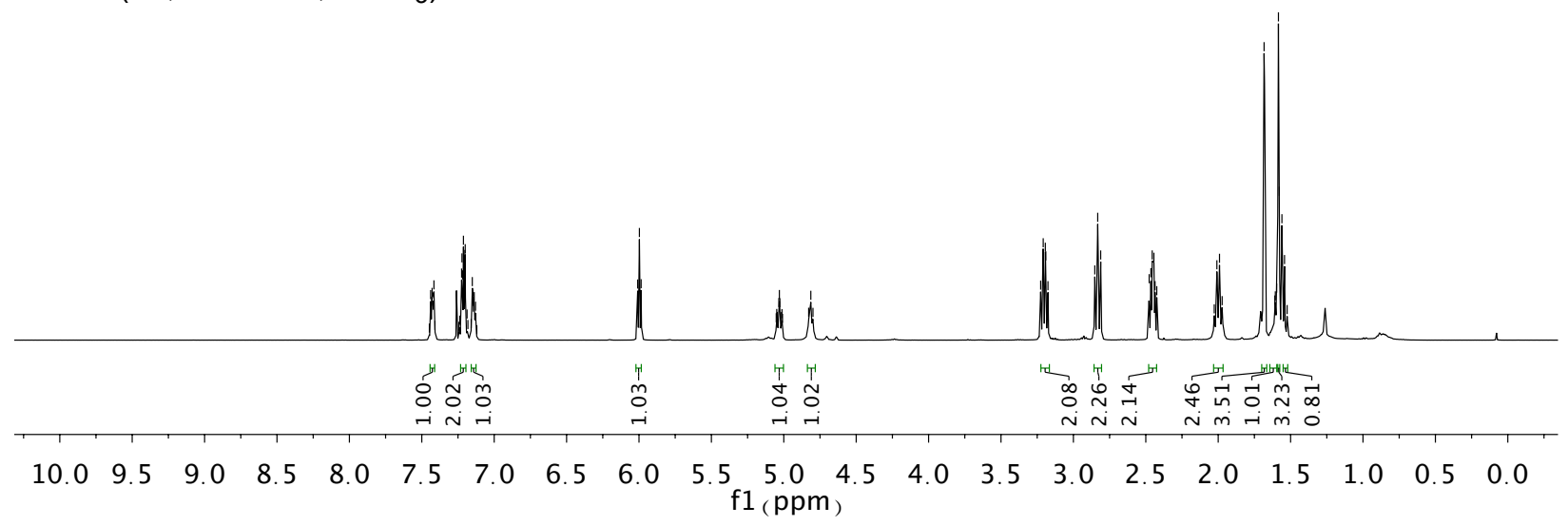

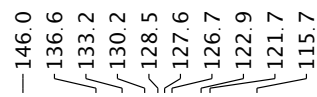

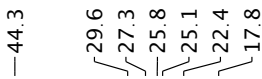<smiles>CC(C)=CCCCNS(=O)(=O)OC1=CCCc2ccccc21</smiles>

10

$\left({ }^{13} \mathrm{C}, 126 \mathrm{MHz}, \mathrm{CDCl}_{3}\right)$

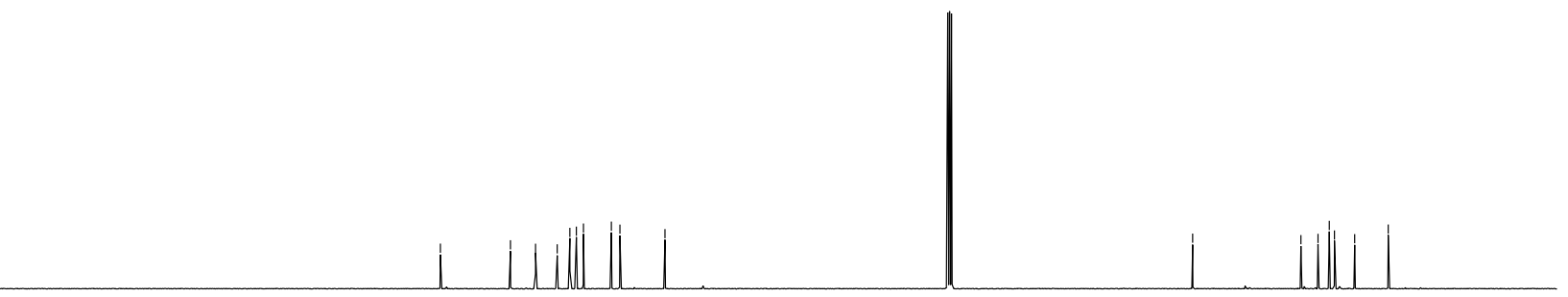

$\begin{array}{lllllllllllllllllllll}1 & 100 & 190 & 180 & 170 & 160 & 150 & 140 & 130 & 120 & 110 \underset{\mathrm{f} 1(\mathrm{ppm})}{100} & 90 & 80 & 70 & 60 & 50 & 40 & 30 & 20 & 10 & 0\end{array}$




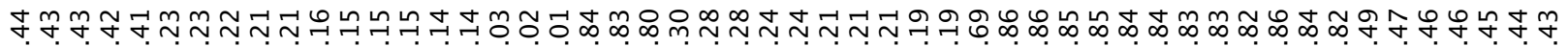

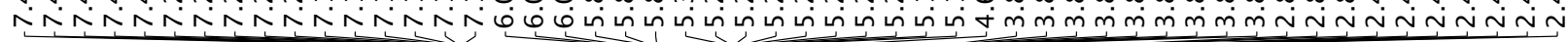<smiles>C=CCNS(=O)(=O)OC1=CCCc2ccccc21</smiles>

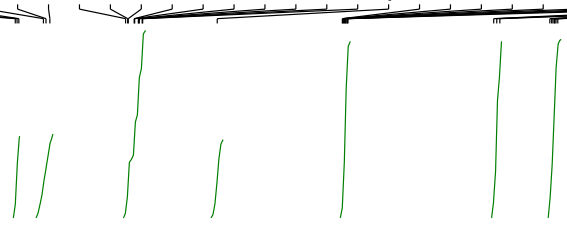

11

$\left({ }^{1} \mathrm{H}, 400 \mathrm{MHz}, \mathrm{CDCl}_{3}\right)$

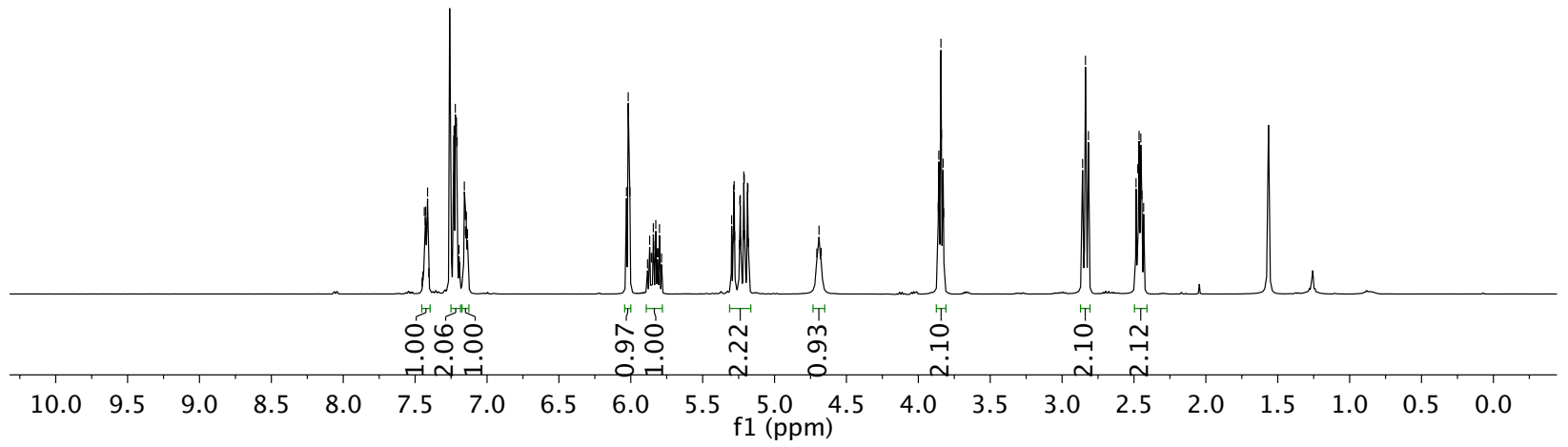

فำ

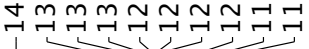<smiles>C=CCNS(=O)(=O)OC1=CCCc2ccccc21</smiles>

11

$\left({ }^{13} \mathrm{C}, 126 \mathrm{MHz}, \mathrm{CDCl}_{3}\right)$

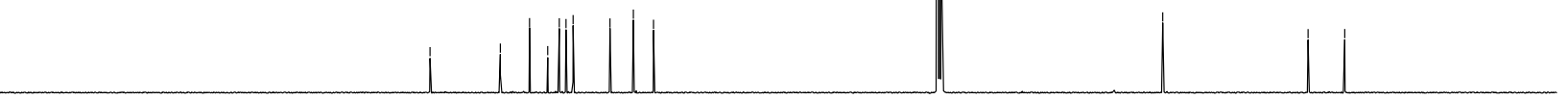

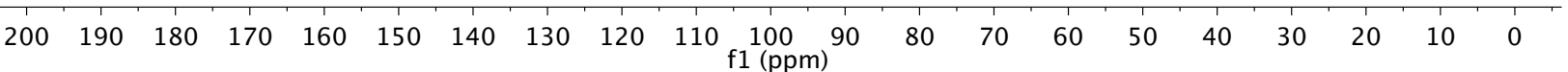




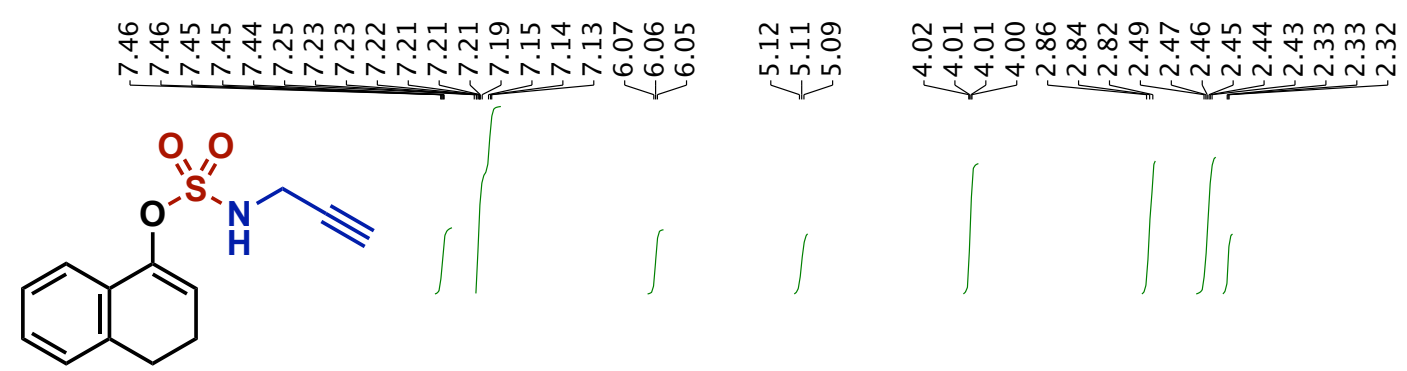

12

$\left({ }^{1} \mathrm{H}, 400 \mathrm{MHz}, \mathrm{CDCl}_{3}\right)$
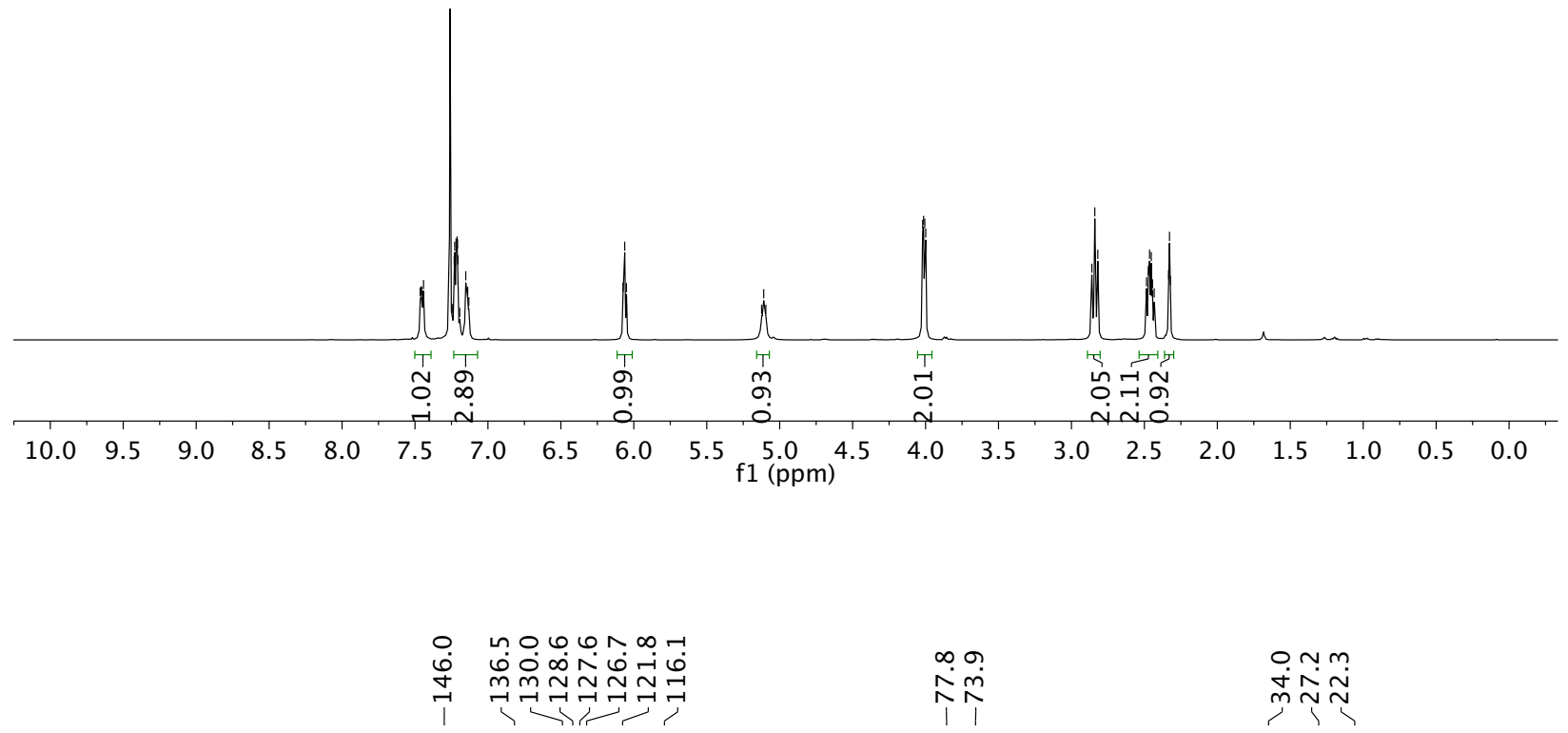

$\stackrel{\infty}{N} \underset{i}{\stackrel{9}{i}}$

mNㅗำ<smiles>C#CCNS(=O)(=O)OC1=CCCc2ccccc21</smiles>

12

$\left({ }^{13} \mathrm{C}, 126 \mathrm{MHz}, \mathrm{CDCl}_{3}\right)$

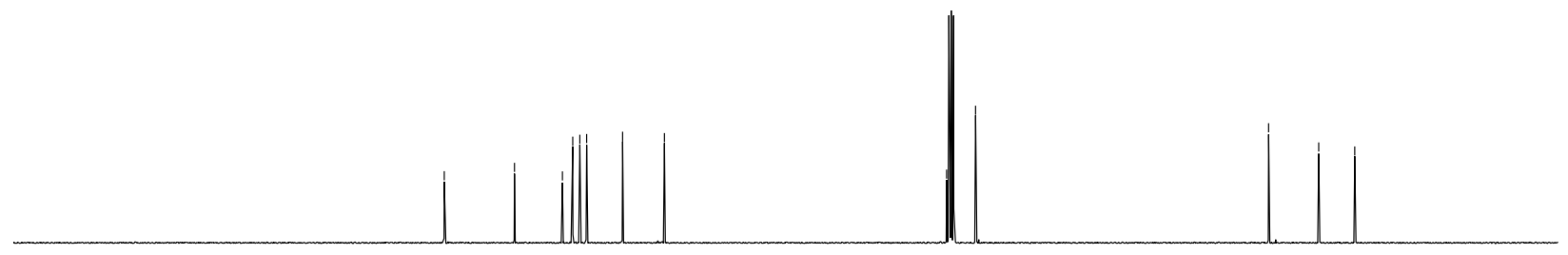

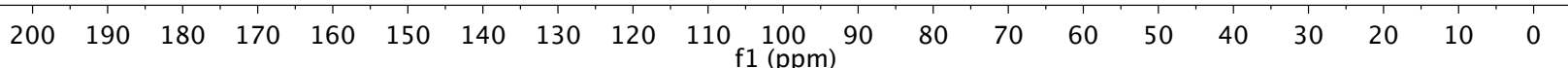




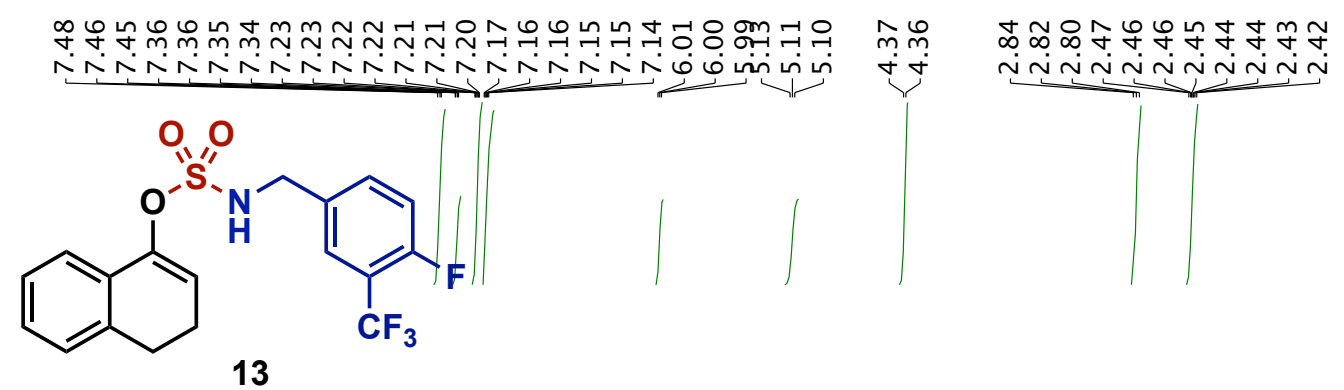

$\left({ }^{1} \mathrm{H}, 400 \mathrm{MHz}, \mathrm{CDCl}_{3}\right)$

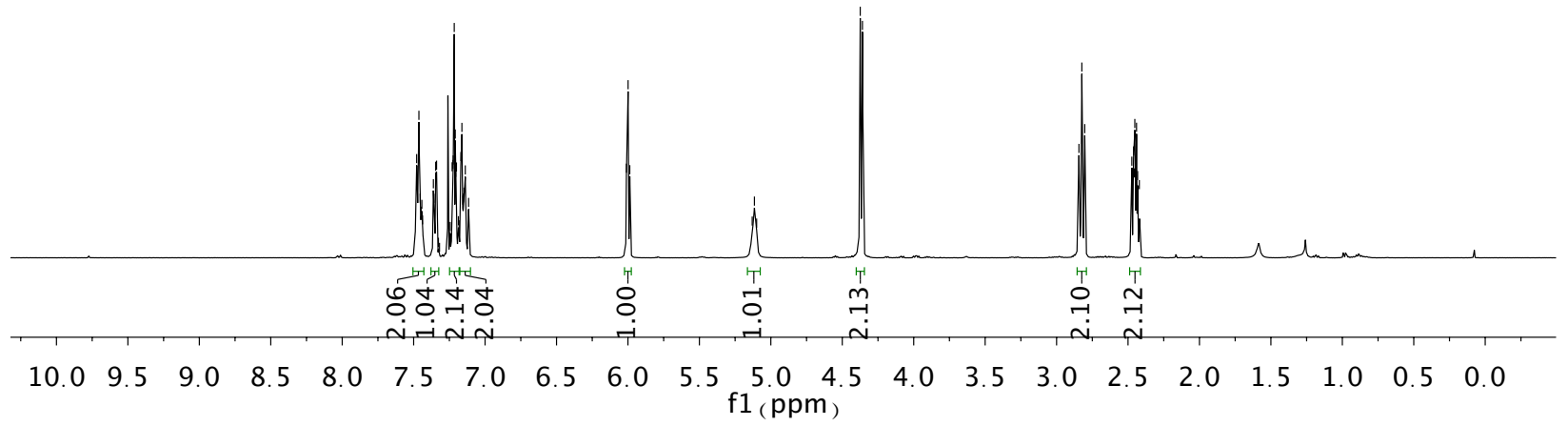

ம

0 $\infty$ i 범듬<smiles>O=S(=O)(NCc1ccc(F)c(C(F)(F)F)c1)OC1=CCCc2ccccc21</smiles>

13

$\left({ }^{13} \mathrm{C}, 126 \mathrm{MHz}, \mathrm{CDCl}_{3}\right)$

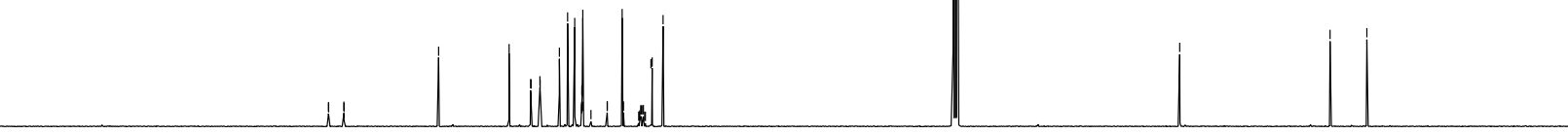

$\begin{array}{lllllllllllllllllllll}1200 & 190 & 180 & 170 & 160 & 150 & 140 & 130 & 120 & 110 \underset{\mathrm{f} 1(\mathrm{ppm})}{100} & 90 & 80 & 70 & 60 & 50 & 40 & 30 & 20 & 10 & 0\end{array}$


<smiles>O=S(=O)(NCc1ccc(F)c(C(F)(F)F)c1)OC1=CCCc2ccccc21</smiles>

13

$\left({ }^{19} \mathrm{~F}, 470 \mathrm{MHz}, \mathrm{CDCl}_{3}\right)$

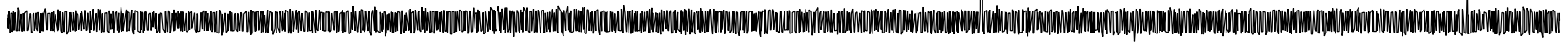

\begin{tabular}{|c|c|c|c|c|c|c|c|c|c|c|c|c|c|c|c|}
\hline 40 & 30 & 20 & 10 & 0 & -10 & -20 & -30 & $\mathrm{f} 1 \begin{array}{l}-40 \\
(\mathrm{ppm})\end{array}$ & -50 & -60 & -70 & -80 & -90 & -100 & $\begin{array}{ll}-110 & -120\end{array}$ \\
\hline
\end{tabular}




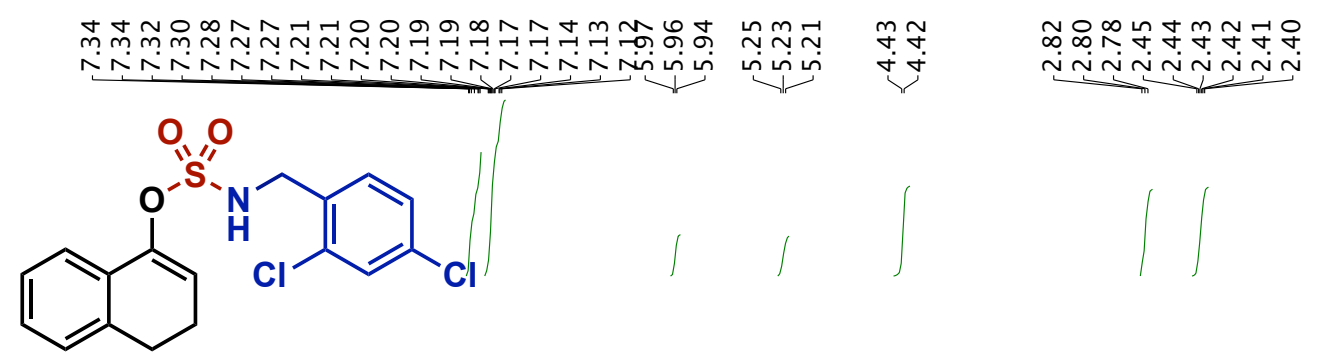

14

$\left({ }^{1} \mathrm{H}, 400 \mathrm{MHz}, \mathrm{CDCl}_{3}\right)$
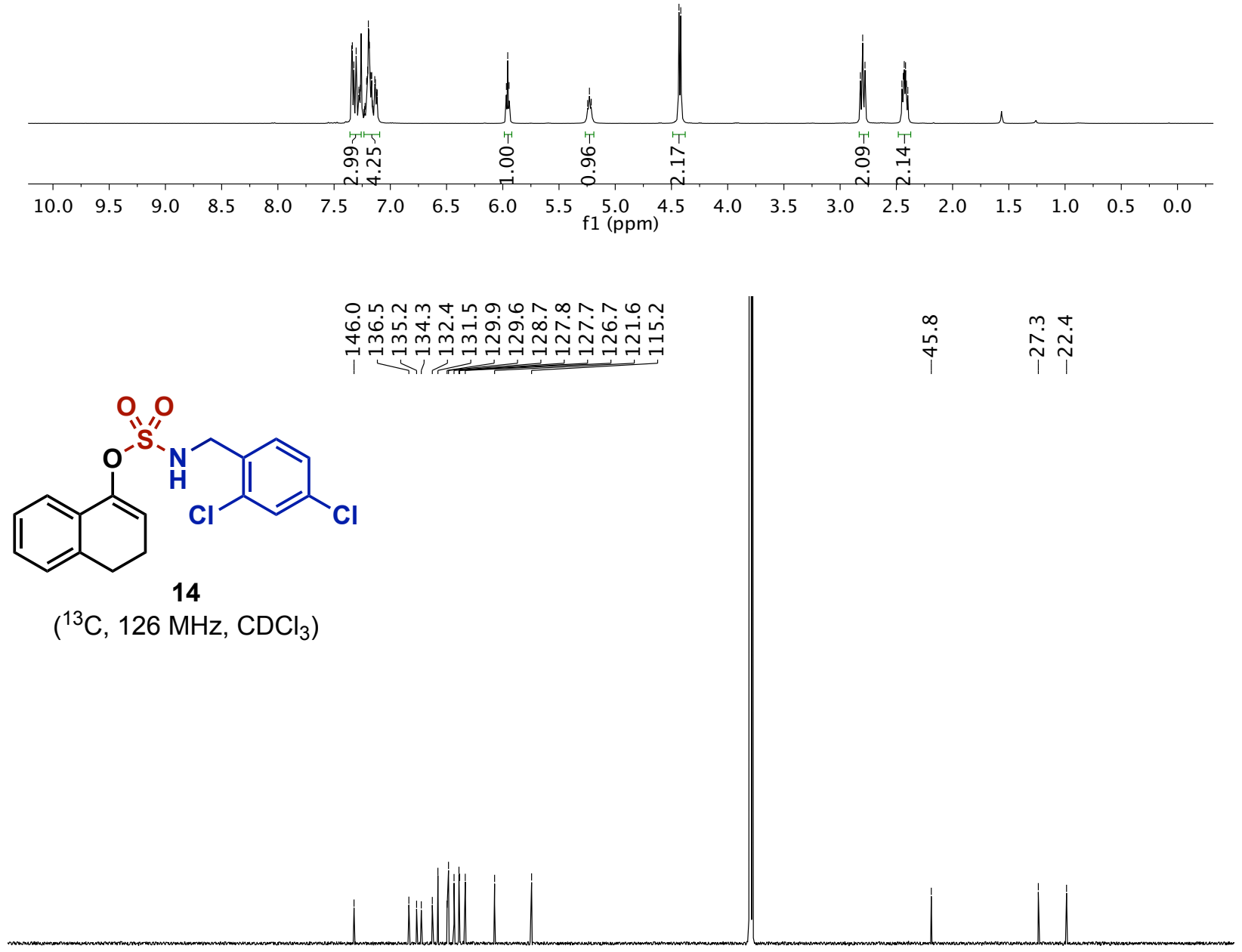

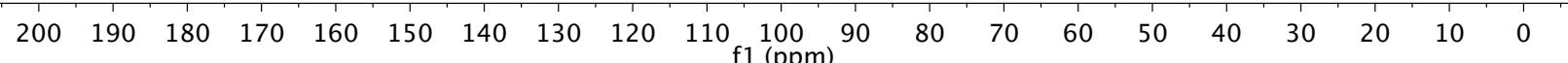




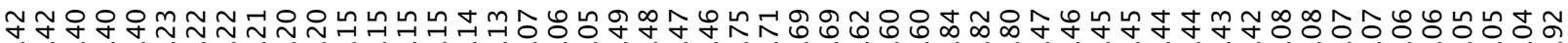

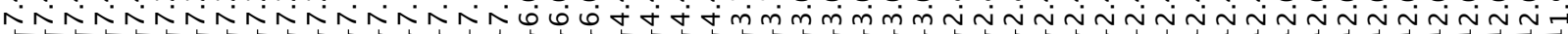

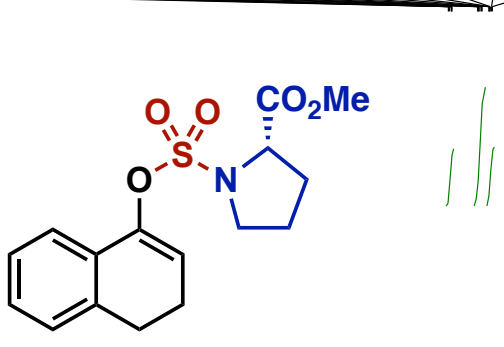

15

$\left({ }^{1} \mathrm{H}, 400 \mathrm{MHz}, \mathrm{CDCl}_{3}\right)$
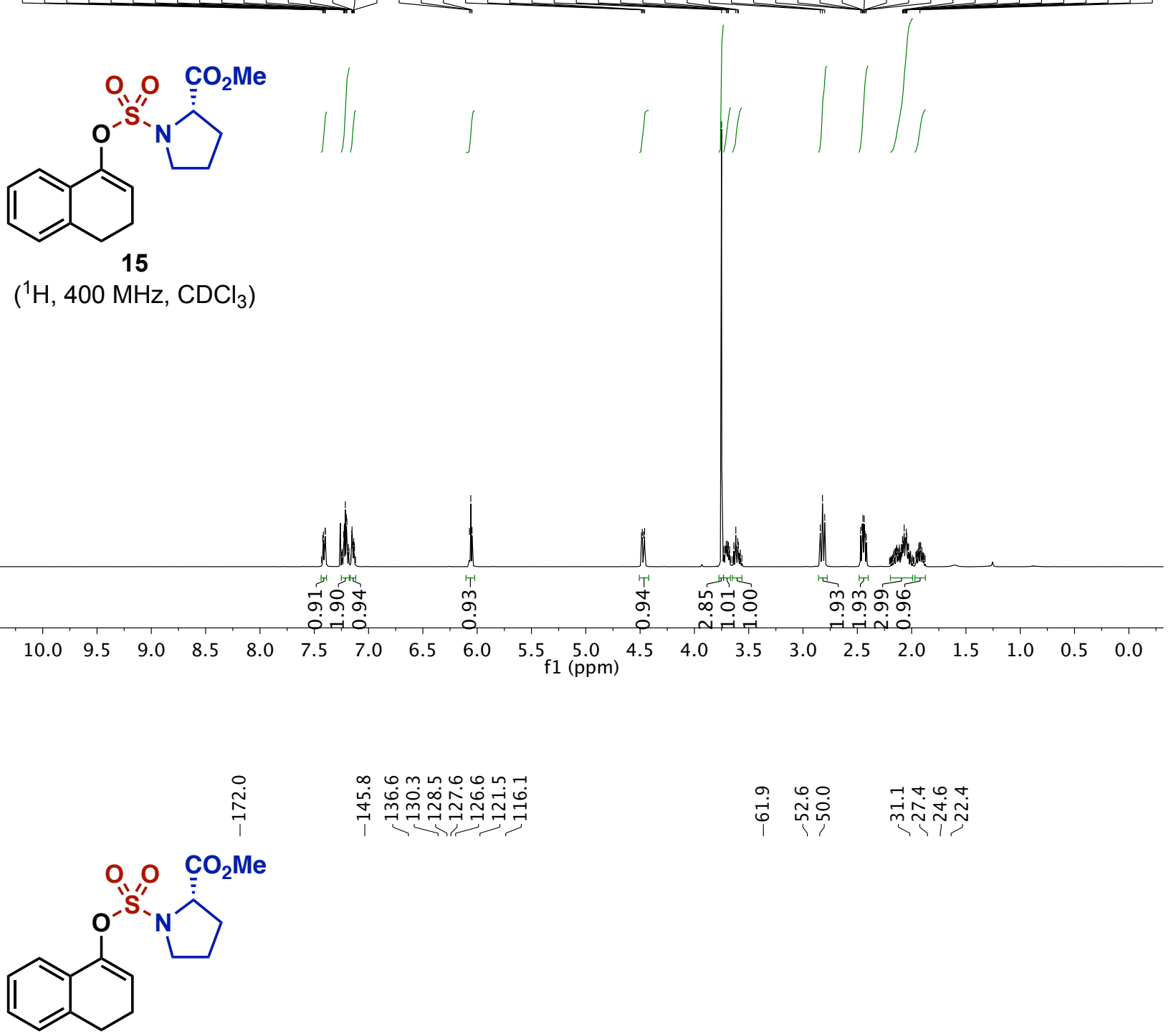

15

$\left({ }^{13} \mathrm{C}, 126 \mathrm{MHz}, \mathrm{CDCl}_{3}\right)$

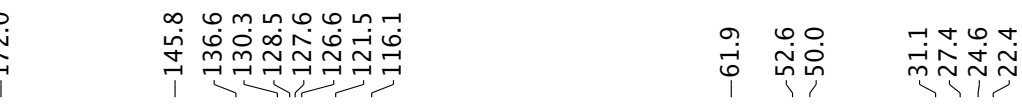

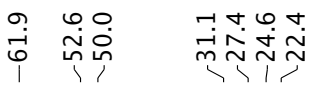
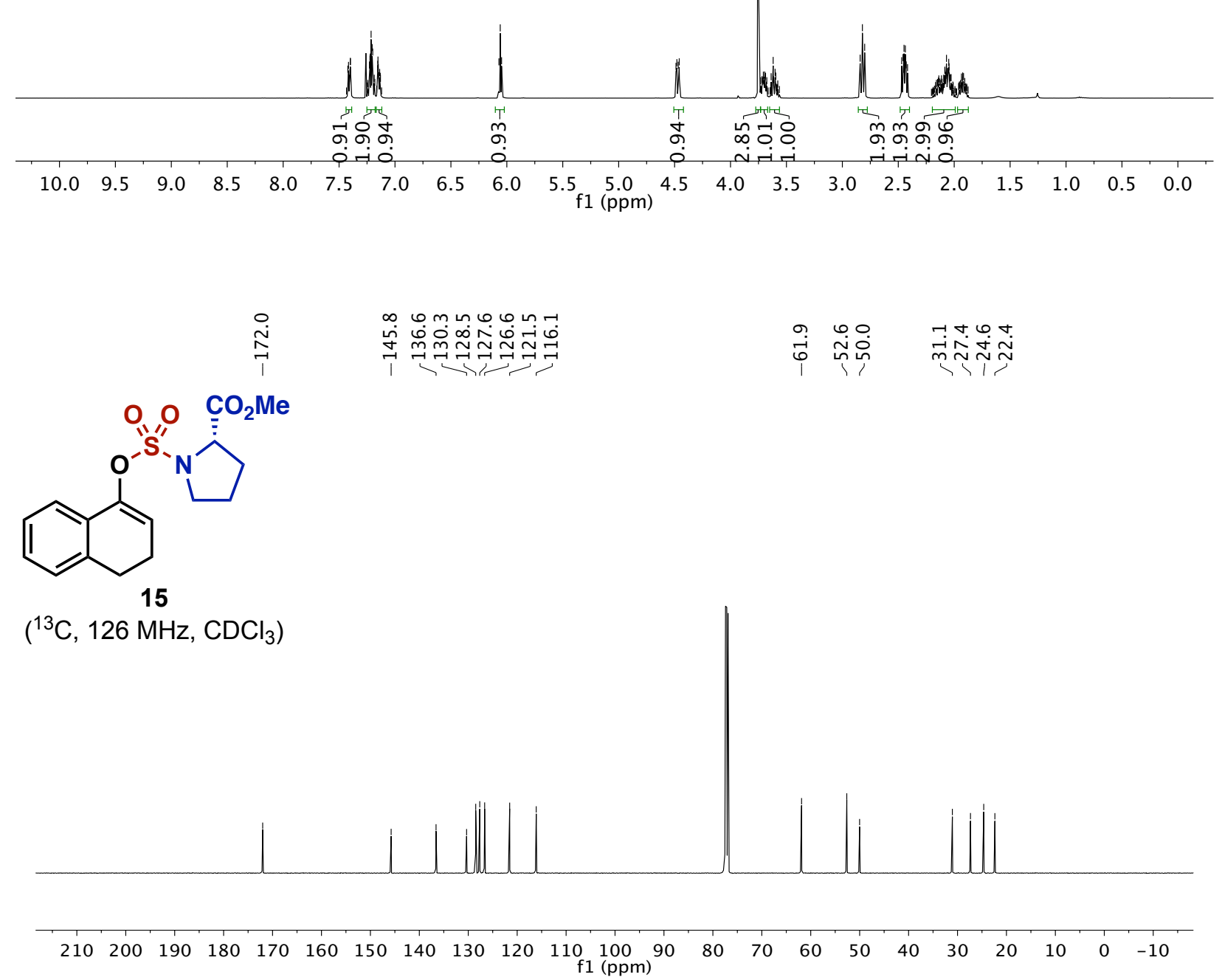


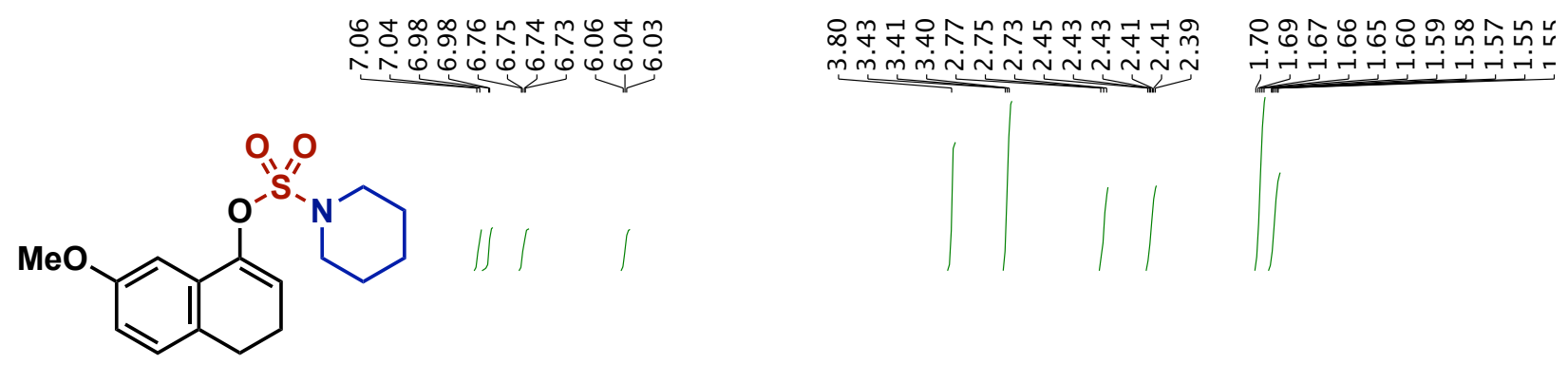

16

$\left({ }^{1} \mathrm{H}, 400 \mathrm{MHz}, \mathrm{CDCl}_{3}\right)$

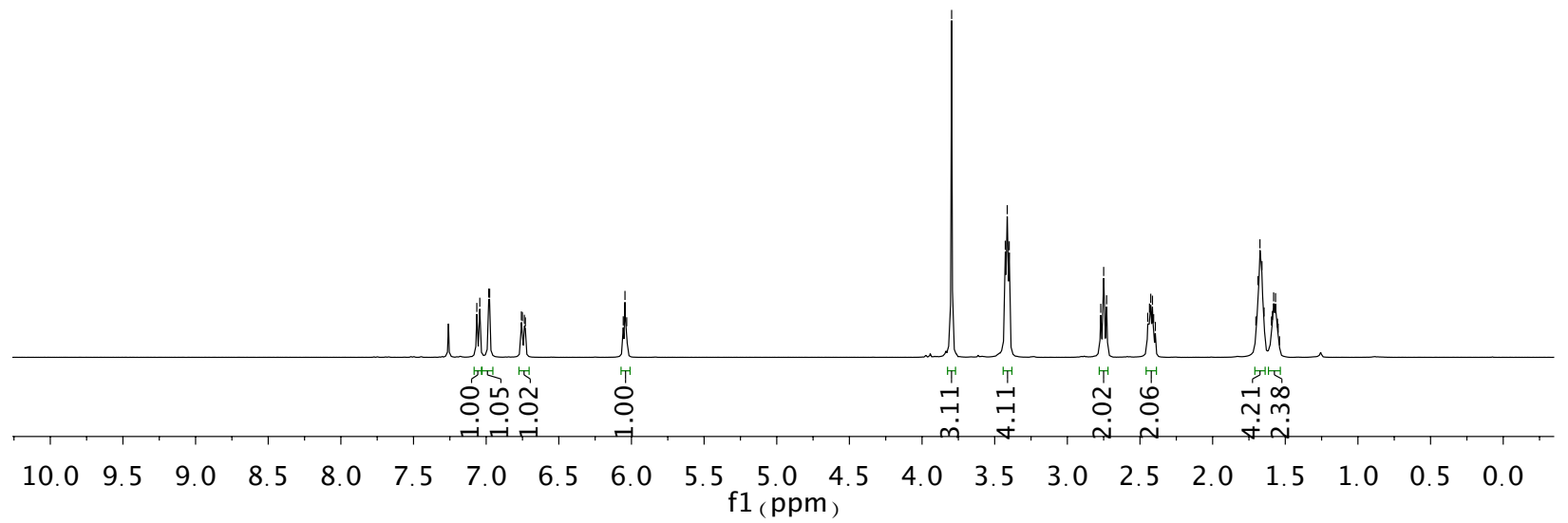

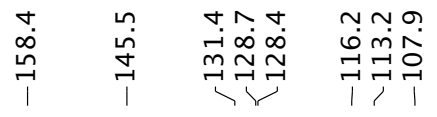

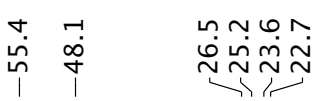<smiles>COc1ccc2c(c1)C(OS(=O)(=O)N1CCCCC1)=CCC2</smiles>

16

$\left({ }^{13} \mathrm{C}, 126 \mathrm{MHz}, \mathrm{CDCl}_{3}\right)$

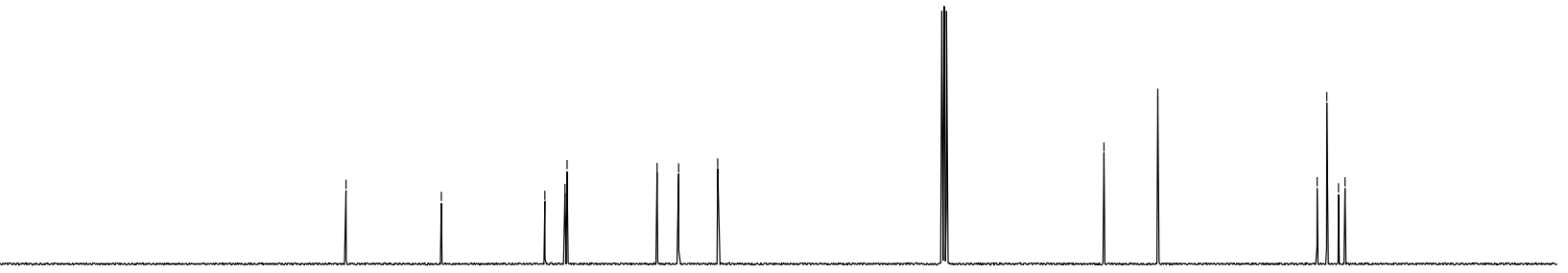

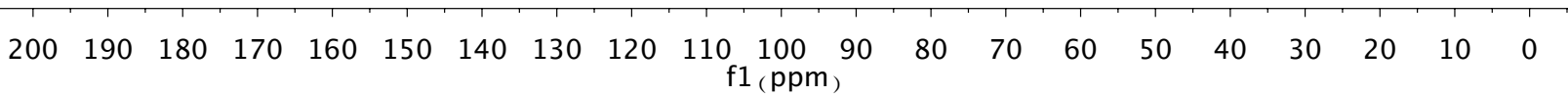




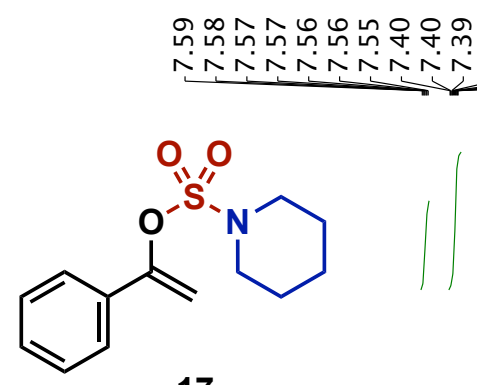

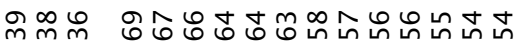

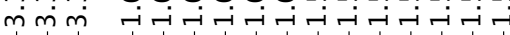

17

$\left({ }^{1} \mathrm{H}, 400 \mathrm{MHz}, \mathrm{CDCl}_{3}\right)$
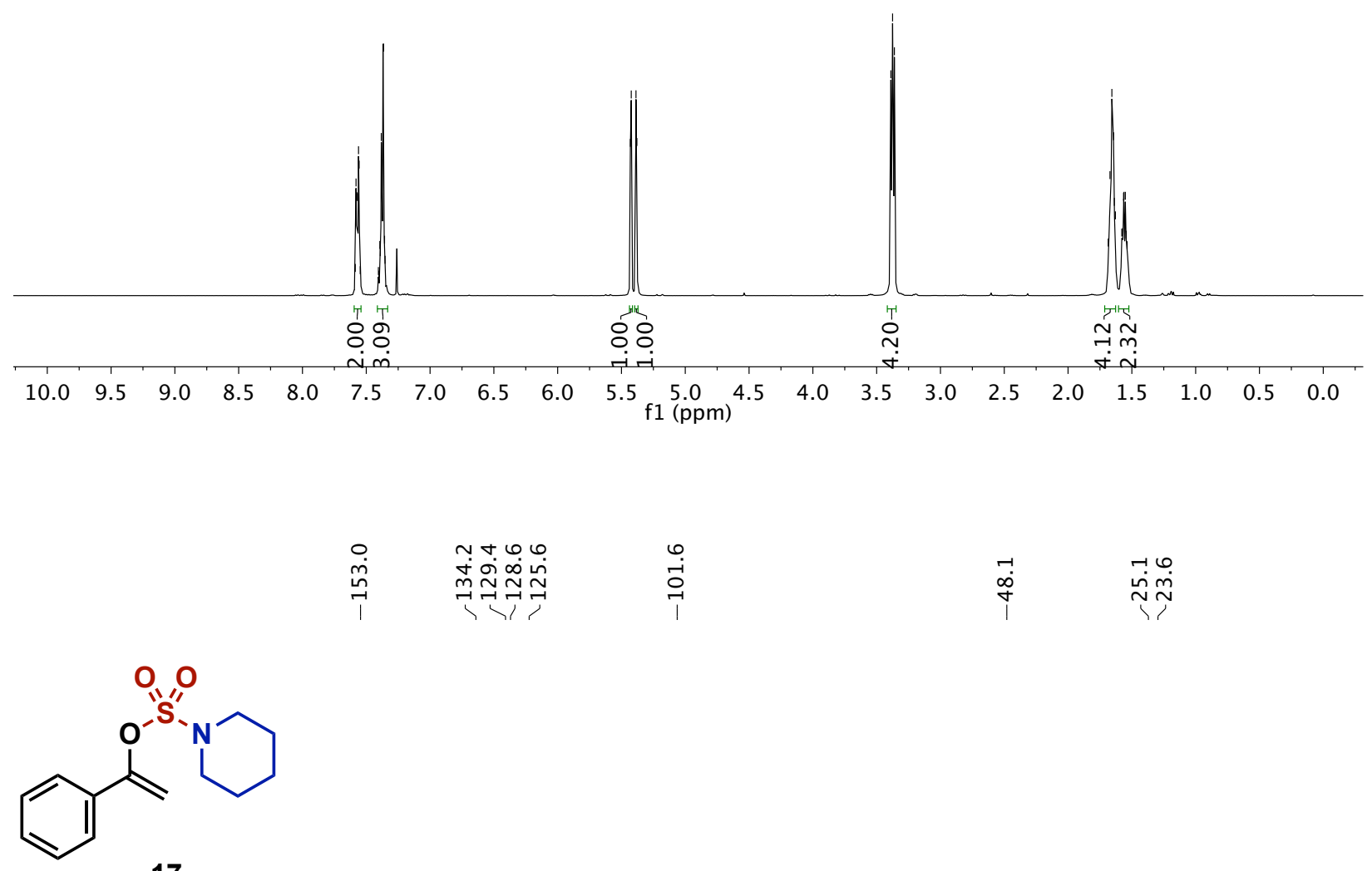

17

$\left({ }^{13} \mathrm{C}, 126 \mathrm{MHz}, \mathrm{CDCl}_{3}\right)$

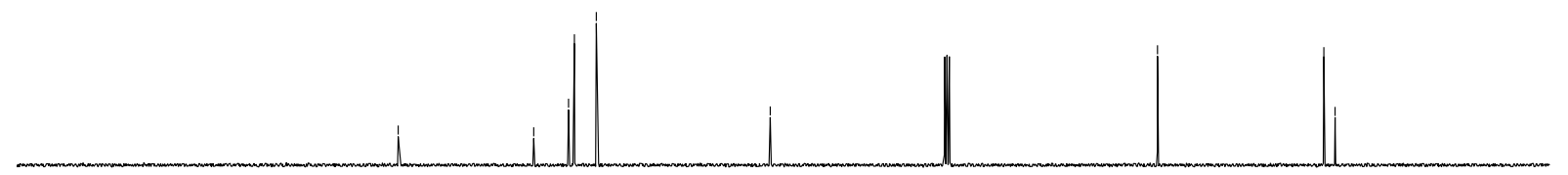

$\begin{array}{lllllllllllllllllllll}200 & 190 & 180 & 170 & 160 & 150 & 140 & 130 & 120 & 110 & 100 & 90 & 80 & 70 & 60 & 50 & 40 & 30 & 20 & 10 & 0\end{array}$ 


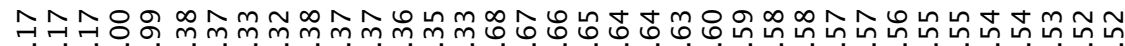

ヘरNं<smiles>C=C(OS(=O)(=O)N1CCCCC1)c1cc(C)cc(C)c1</smiles>

$\left({ }^{1} \mathrm{H}, 400 \mathrm{MHz}, \mathrm{CDCl}_{3}\right)$
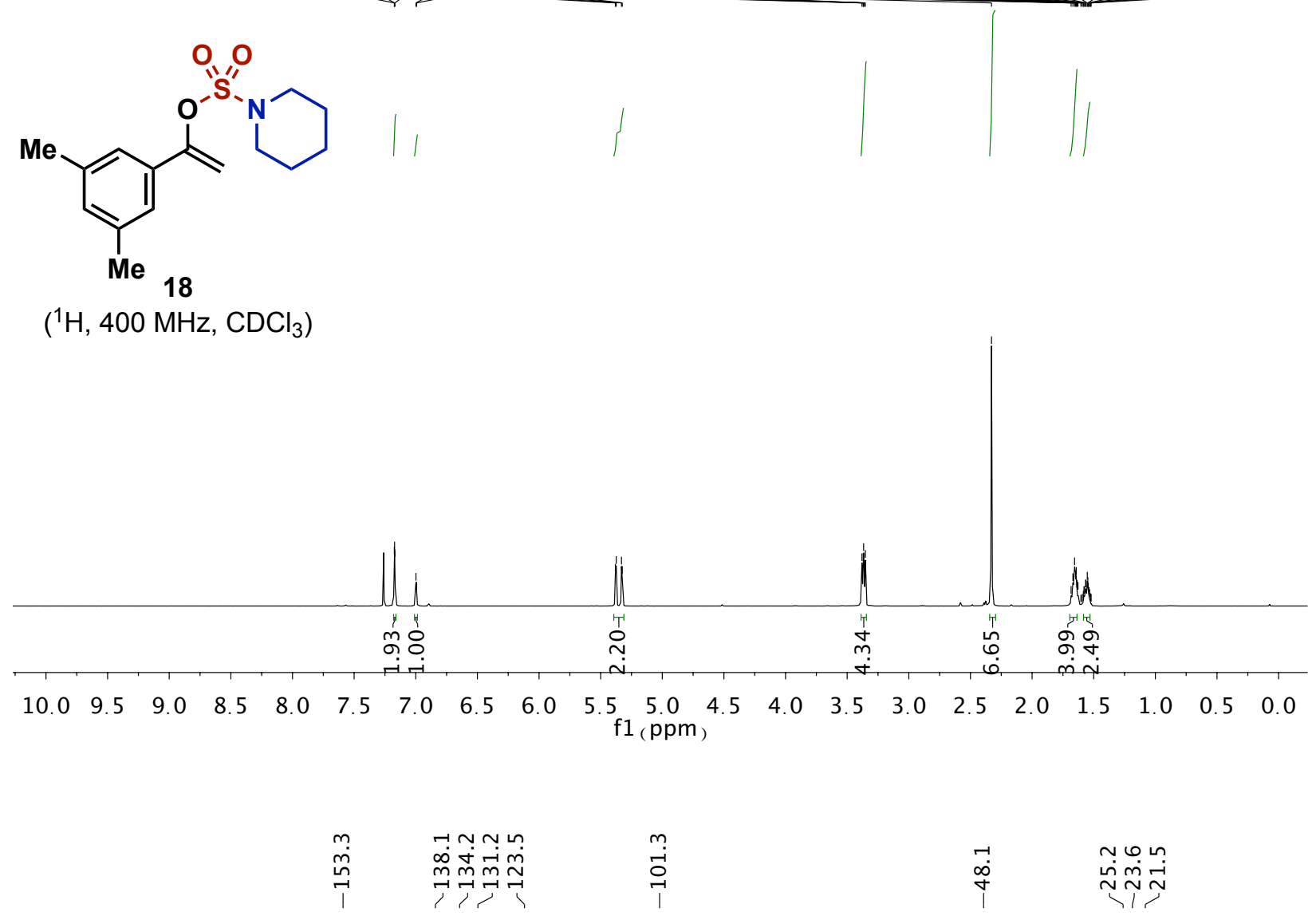<smiles>C=C(OS(=O)(=O)N1CCCCC1)c1cc(C)cc(C)c1</smiles>

$\left({ }^{13} \mathrm{C}, 126 \mathrm{MHz}, \mathrm{CDCl}_{3}\right)$

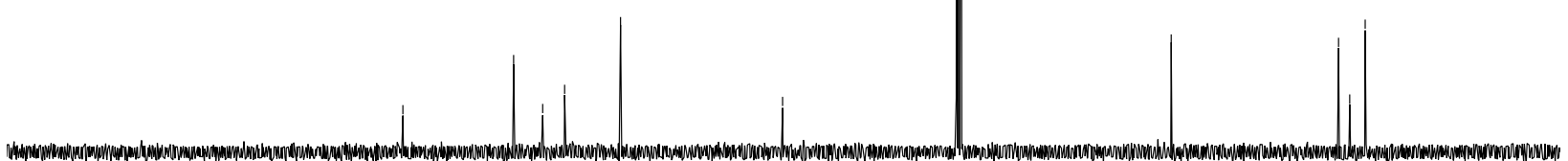

$\begin{array}{llllllllllllllllllll}200 & 190 & 180 & 170 & 160 & 150 & 140 & 130 & 120 & \begin{array}{c}110 \\ \mathrm{f} 1\end{array} \underset{(\mathrm{ppm})}{100} & 90 & 80 & 70 & 60 & 50 & 40 & 30 & 20 & 10 & 0\end{array}$



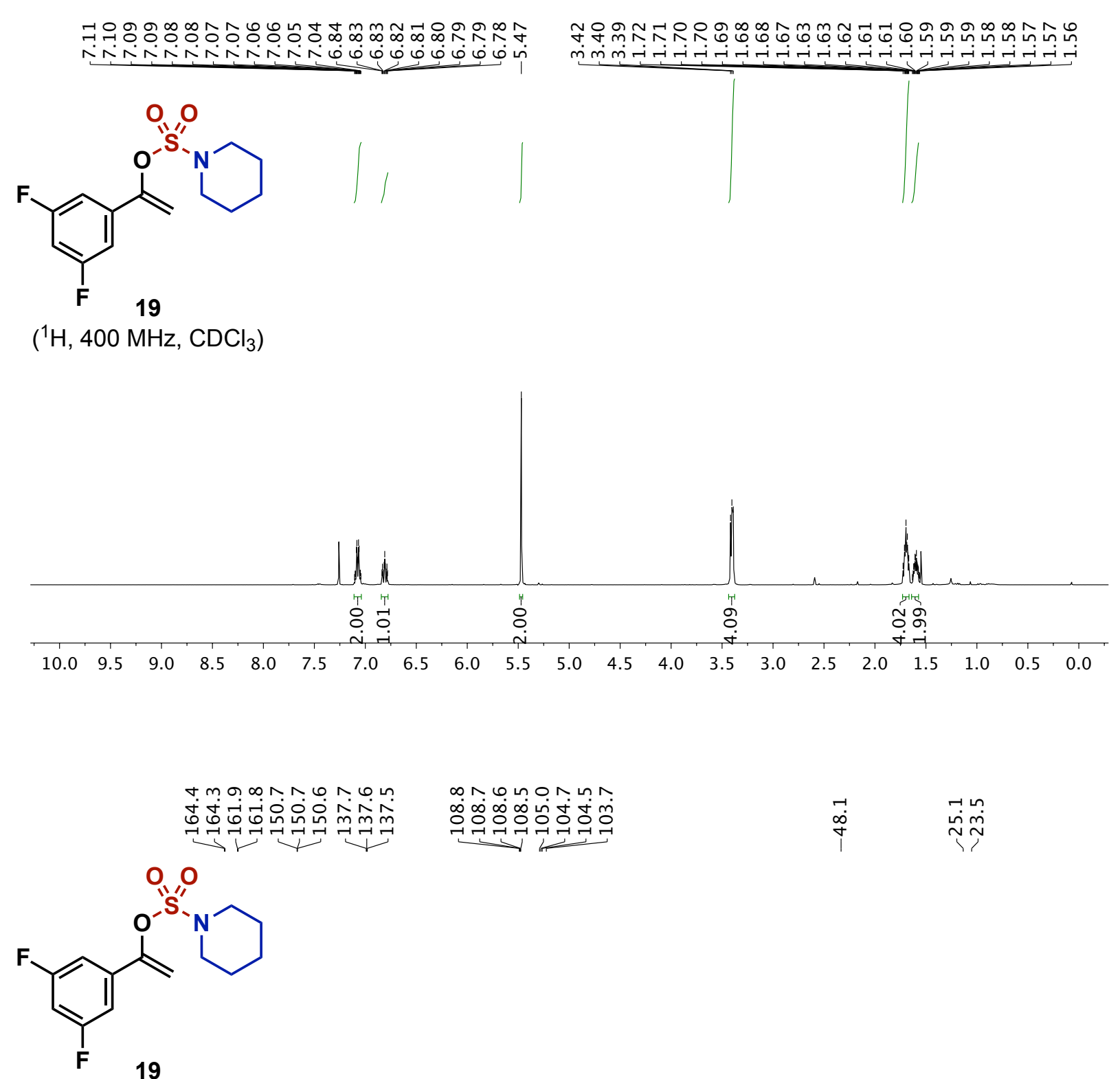

$\left({ }^{13} \mathrm{C}, 126 \mathrm{MHz}, \mathrm{CDCl}_{3}\right)$
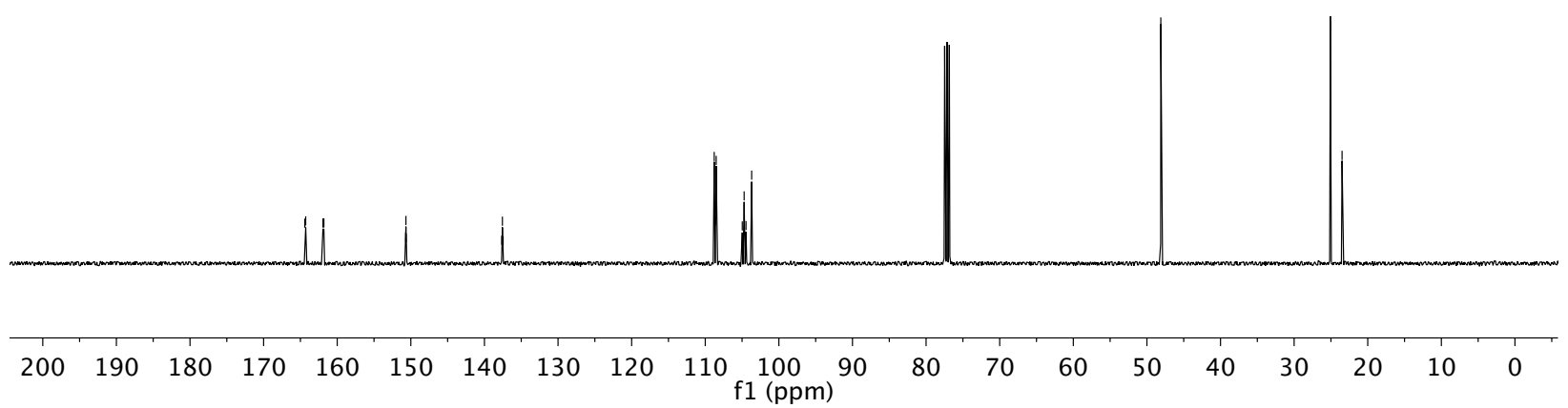
$m m m m$

8.ं8०

111

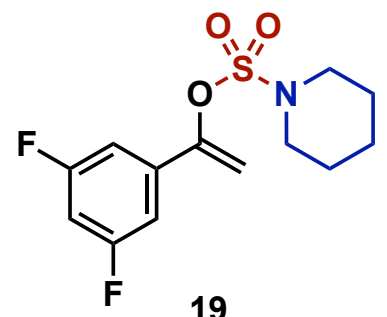

$\left({ }^{19} \mathrm{~F}, 470 \mathrm{MHz}, \mathrm{CDCl}_{3}\right)$

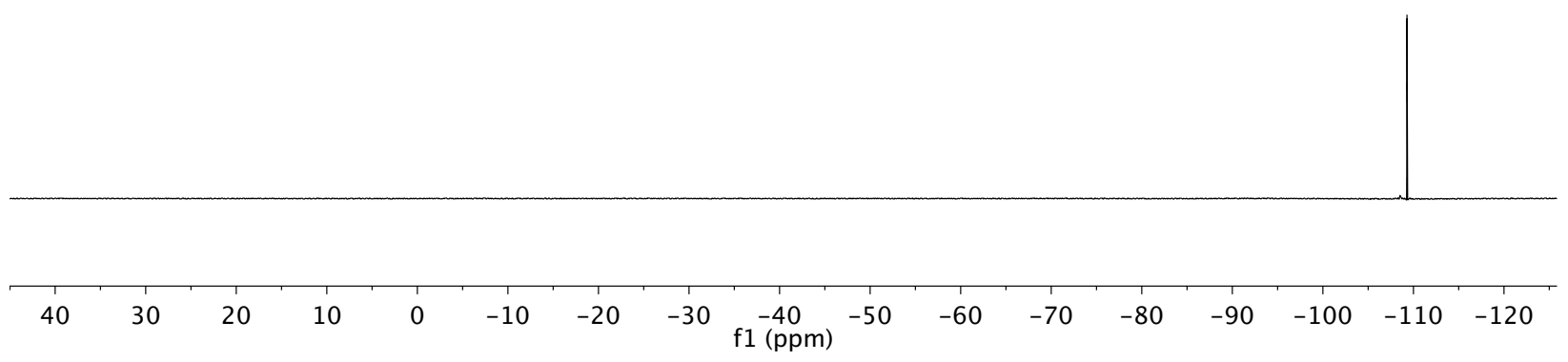




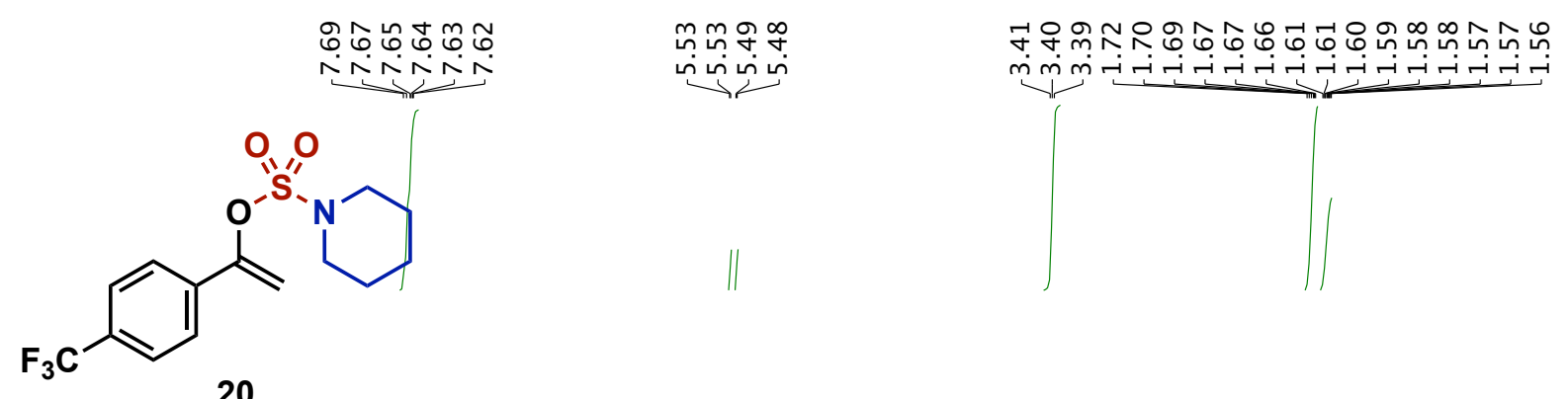

$\left({ }^{1} \mathrm{H}, 400 \mathrm{MHz}, \mathrm{CDCl}_{3}\right)$
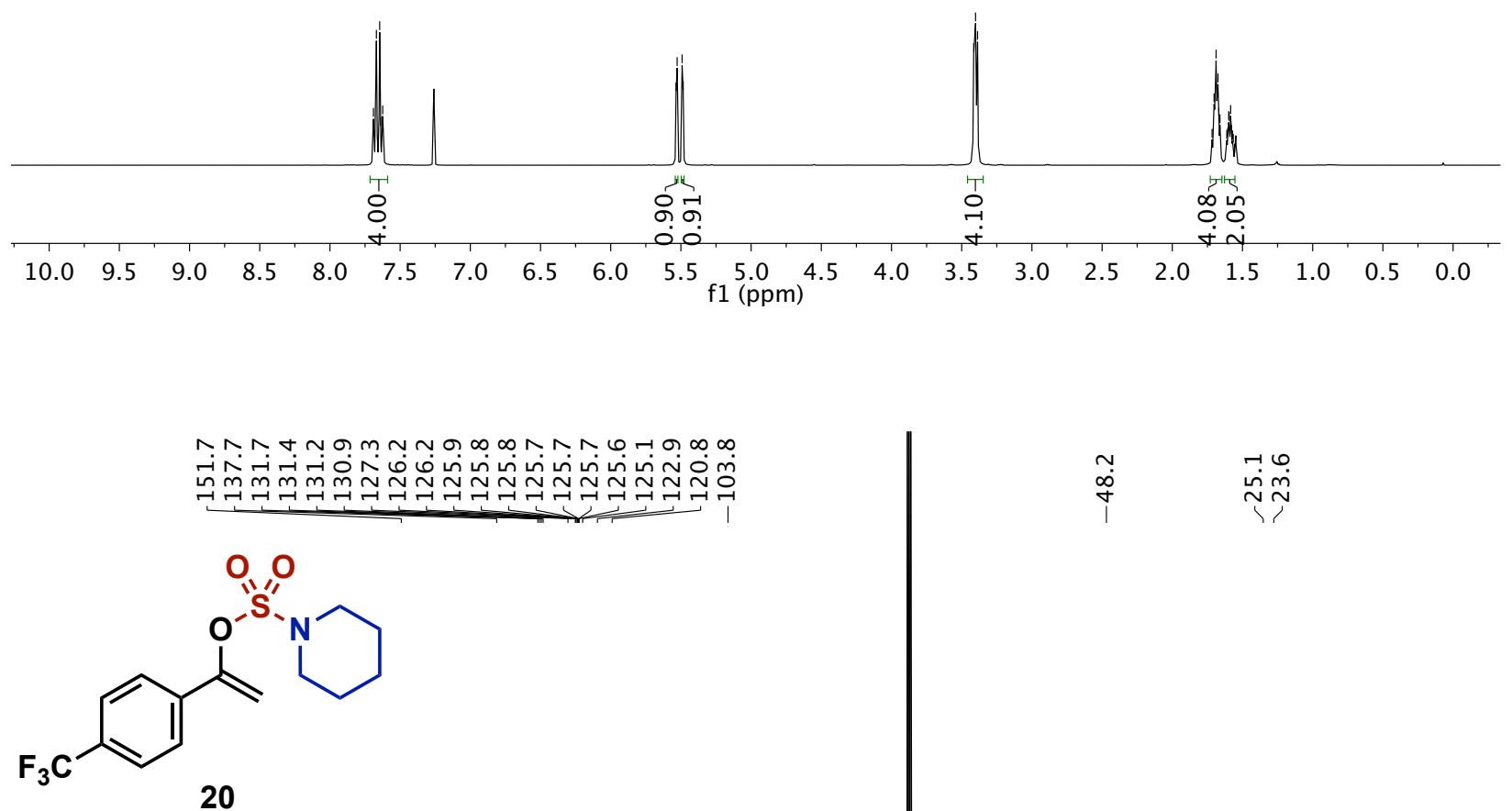

$\left({ }^{13} \mathrm{C}, 126 \mathrm{MHz}, \mathrm{CDCl}_{3}\right)$

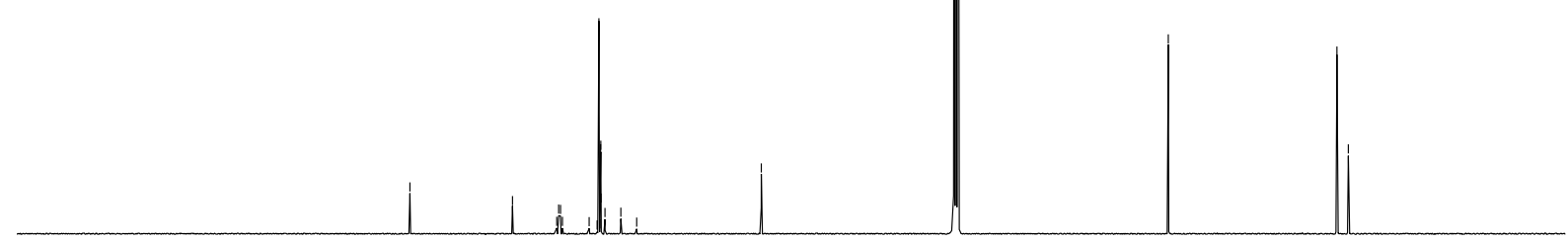

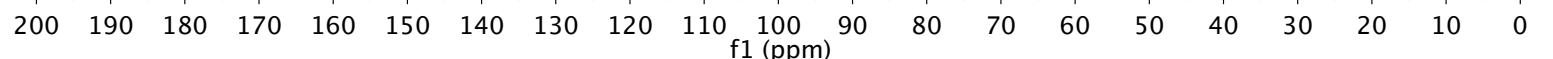




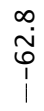<smiles>C=C(OS(=O)(=O)N1CCCCC1)c1ccc(C(F)(F)F)cc1</smiles>

$\left({ }^{19} \mathrm{~F}, 470 \mathrm{MHz}, \mathrm{CDCl}_{3}\right)$

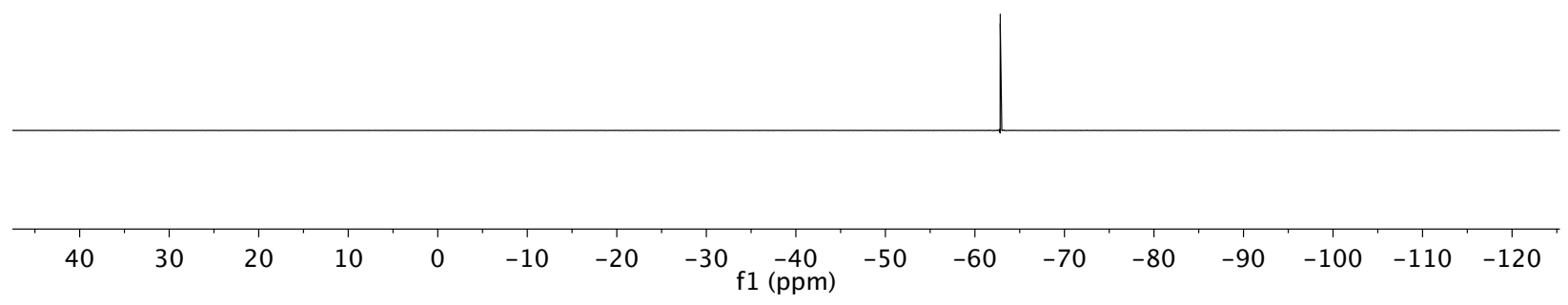




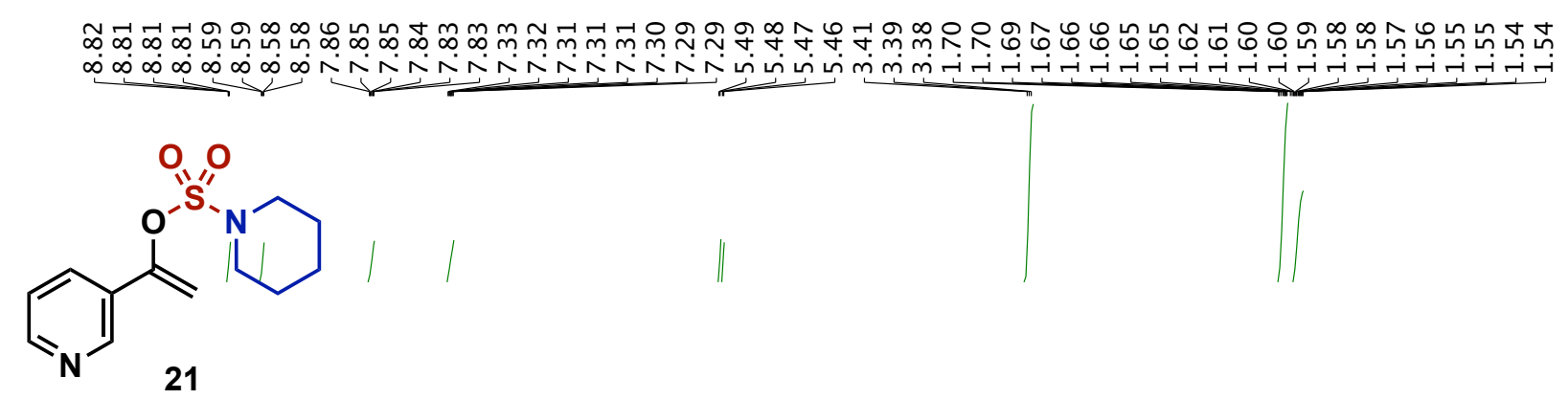

$\left({ }^{1} \mathrm{H}, 400 \mathrm{MHz}, \mathrm{CDCl}_{3}\right)$
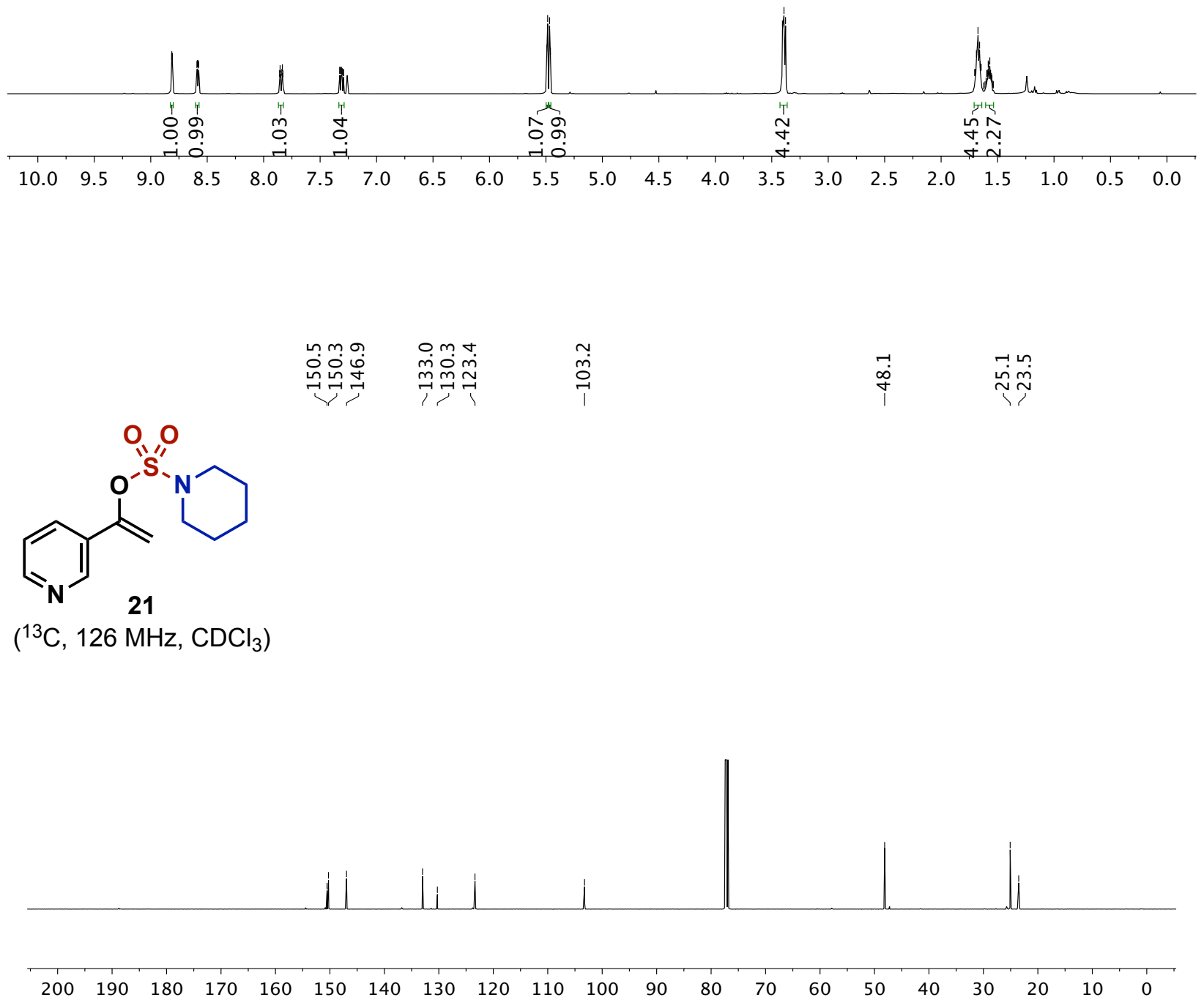


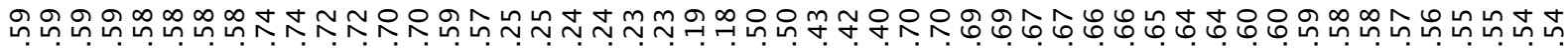

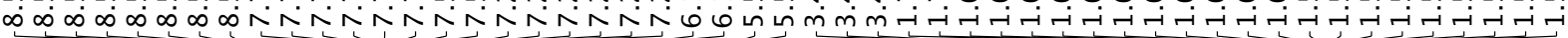<smiles>C=C(OS(=O)(=O)N1CCCCC1)c1ccccn1</smiles>

$\left({ }^{1} \mathrm{H}, 400 \mathrm{MHz}, \mathrm{CDCl}_{3}\right)$

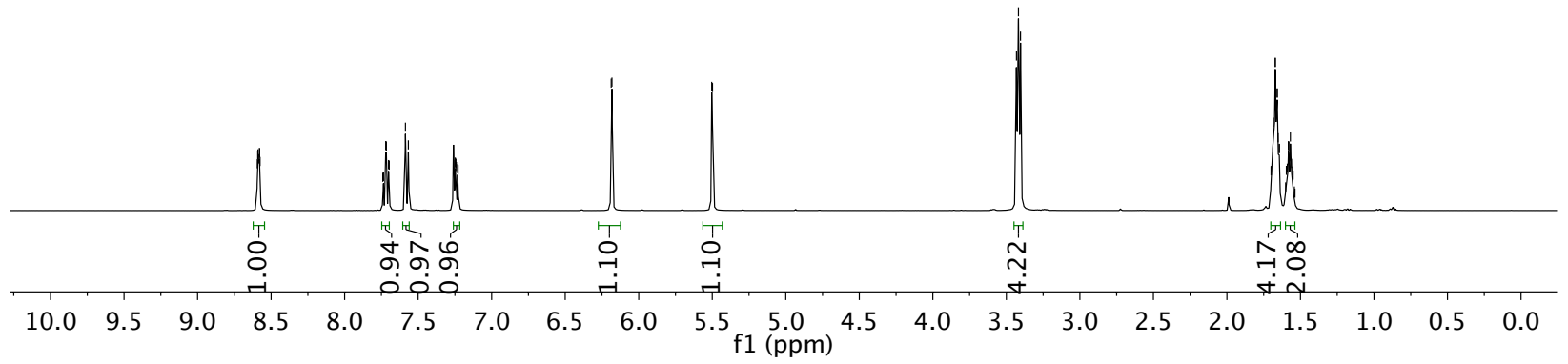

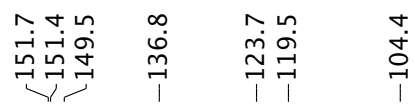

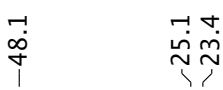<smiles>C=C(OS(=O)(=O)N1CCCCC1)c1ccccn1</smiles>

$\left({ }^{13} \mathrm{C}, 126 \mathrm{MHz}, \mathrm{CDCl}_{3}\right)$

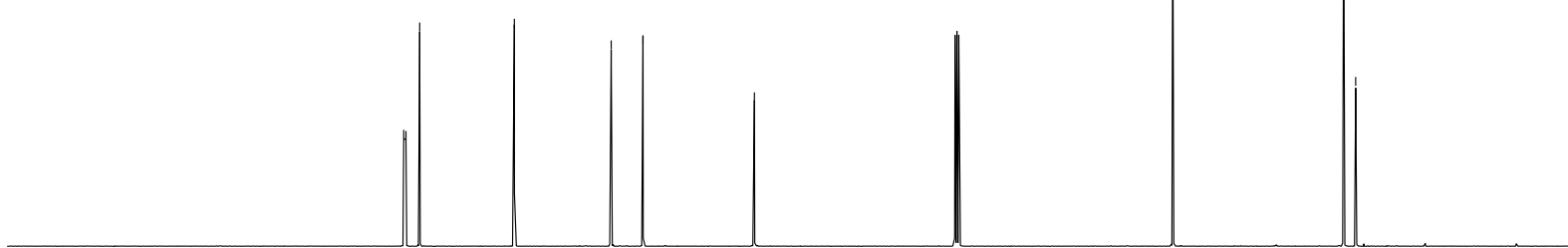

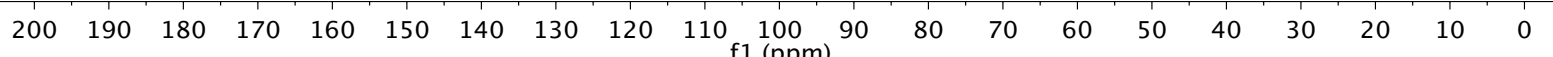




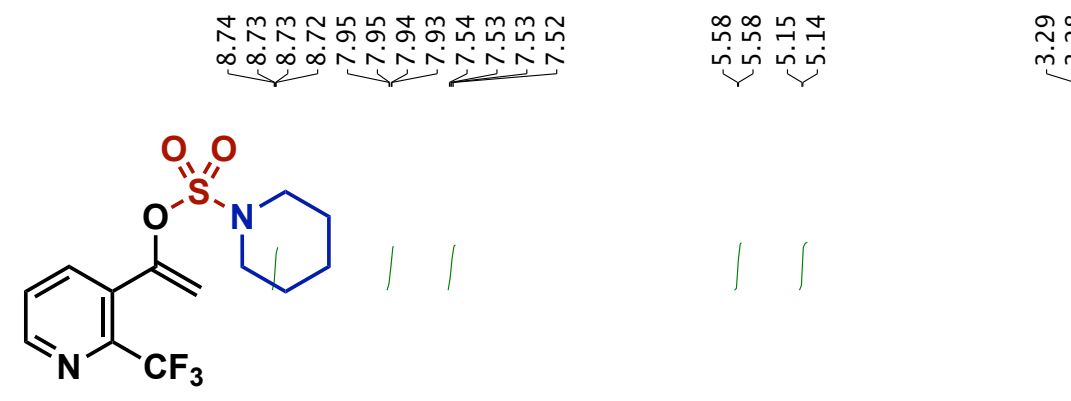

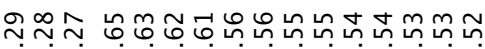

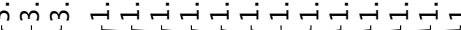

23

$\left({ }^{1} \mathrm{H}, 400 \mathrm{MHz}, \mathrm{CDCl}_{3}\right)$
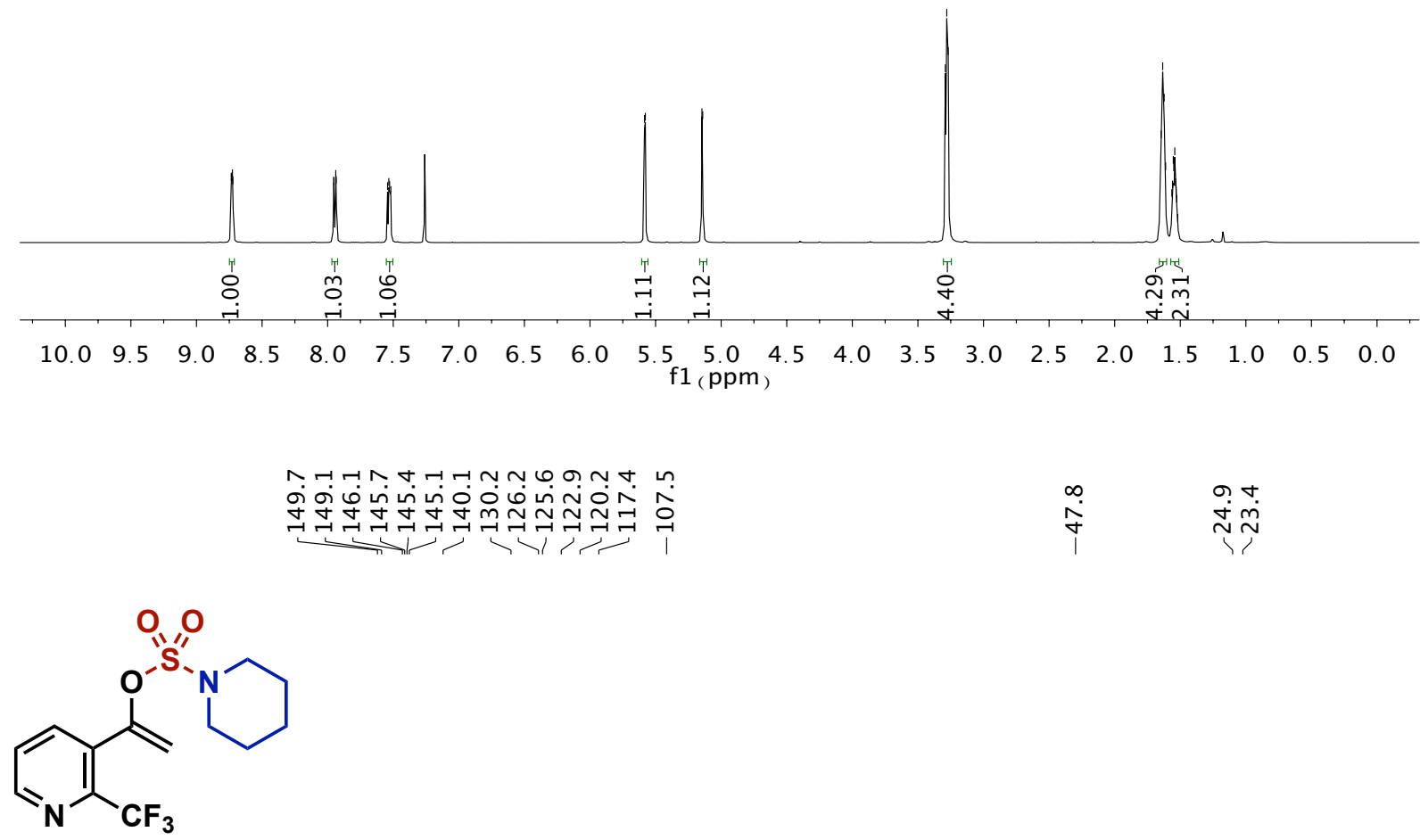

23

$\left({ }^{13} \mathrm{C}, 126 \mathrm{MHz}, \mathrm{CDCl}_{3}\right)$

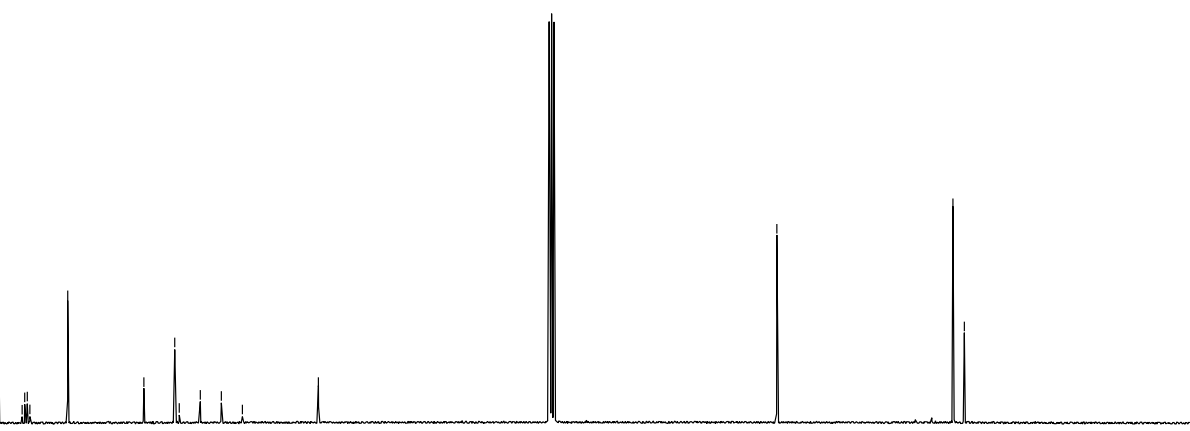

\begin{tabular}{|c|c|c|c|c|c|c|c|c|c|c|c|c|c|c|c|c|c|c|c|c|c|}
\hline 200 & 190 & 180 & 170 & 160 & 150 & 140 & 130 & 120 & & $\begin{array}{r}100 \\
1 \text { (ppr }\end{array}$ & & 80 & 7 & & 6 & & 50 & 40 & 30 & 20 & 10 \\
\hline
\end{tabular}




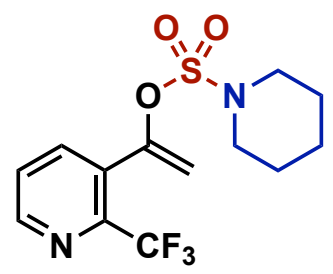

23

$\left({ }^{19} \mathrm{~F}, 470 \mathrm{MHz}, \mathrm{CDCl}_{3}\right)$

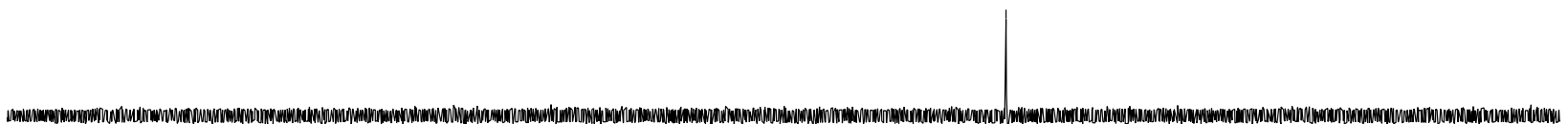

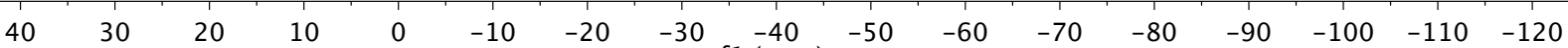




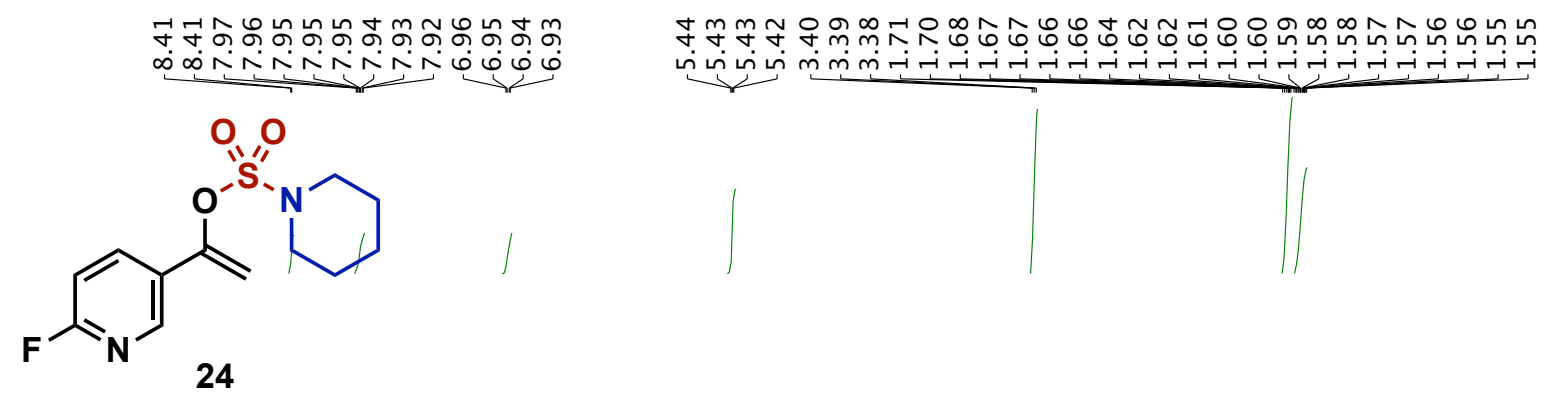

$\left({ }^{1} \mathrm{H}, 400 \mathrm{MHz}, \mathrm{CDCl}_{3}\right)$

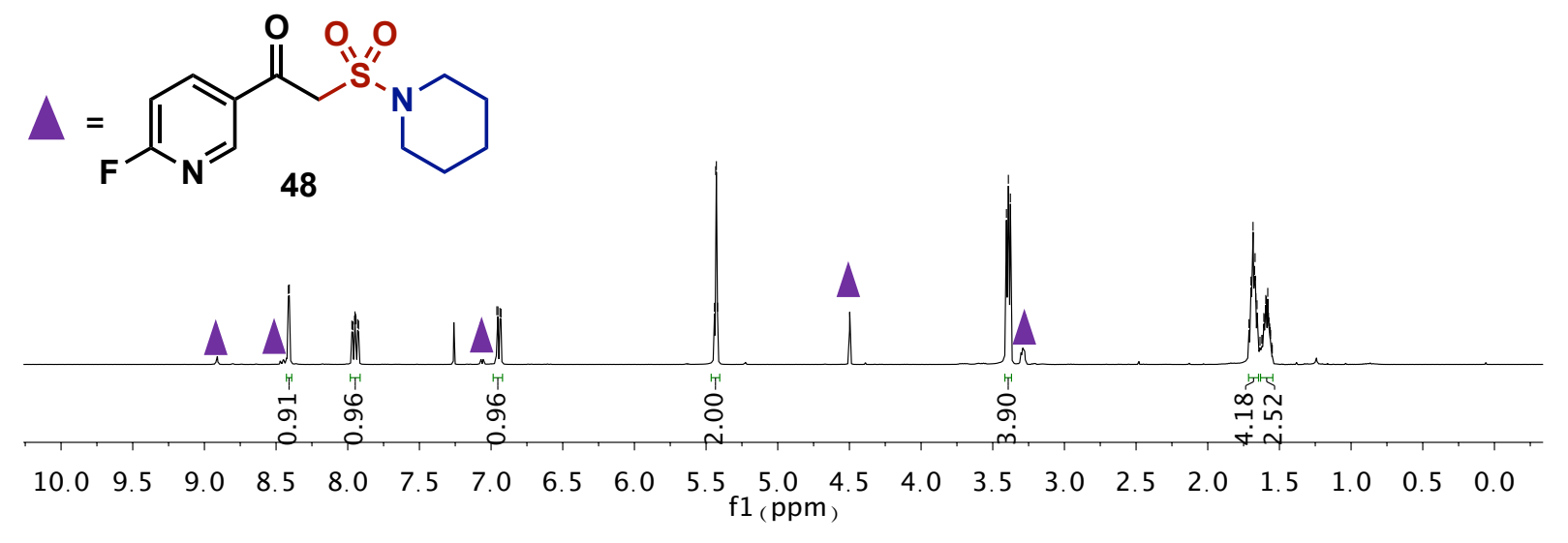

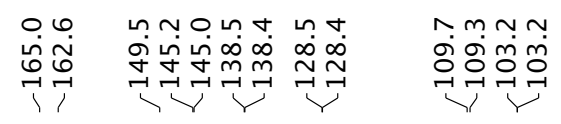
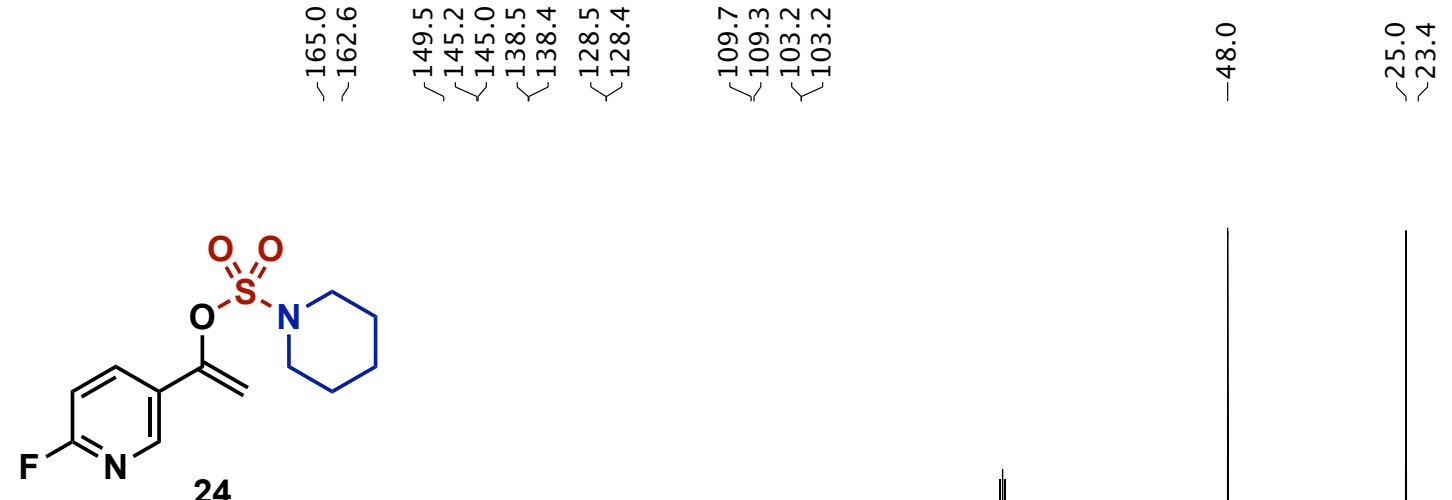

$\left({ }^{13} \mathrm{C}, 126 \mathrm{MHz}, \mathrm{CDCl}_{3}\right)$
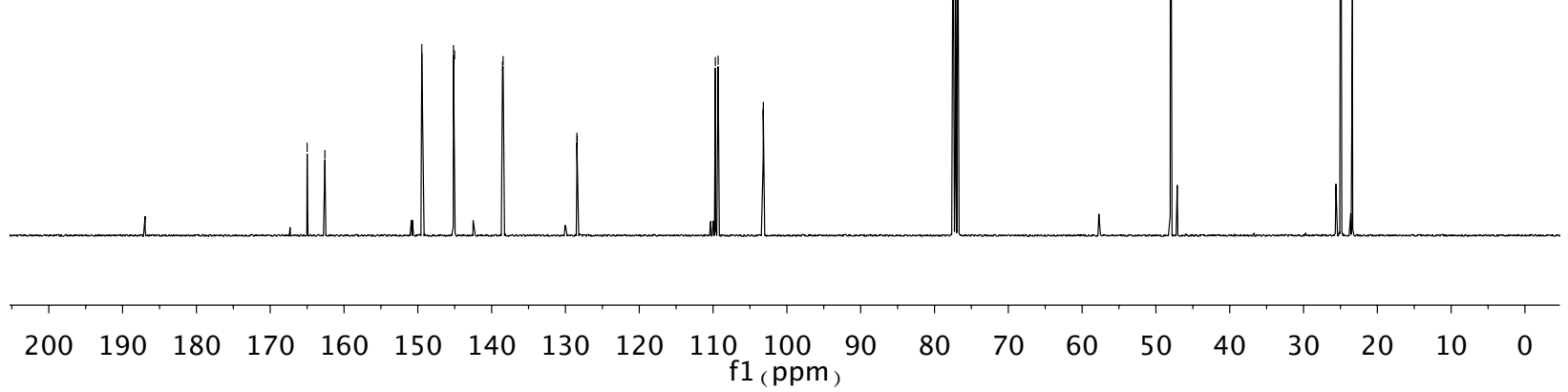


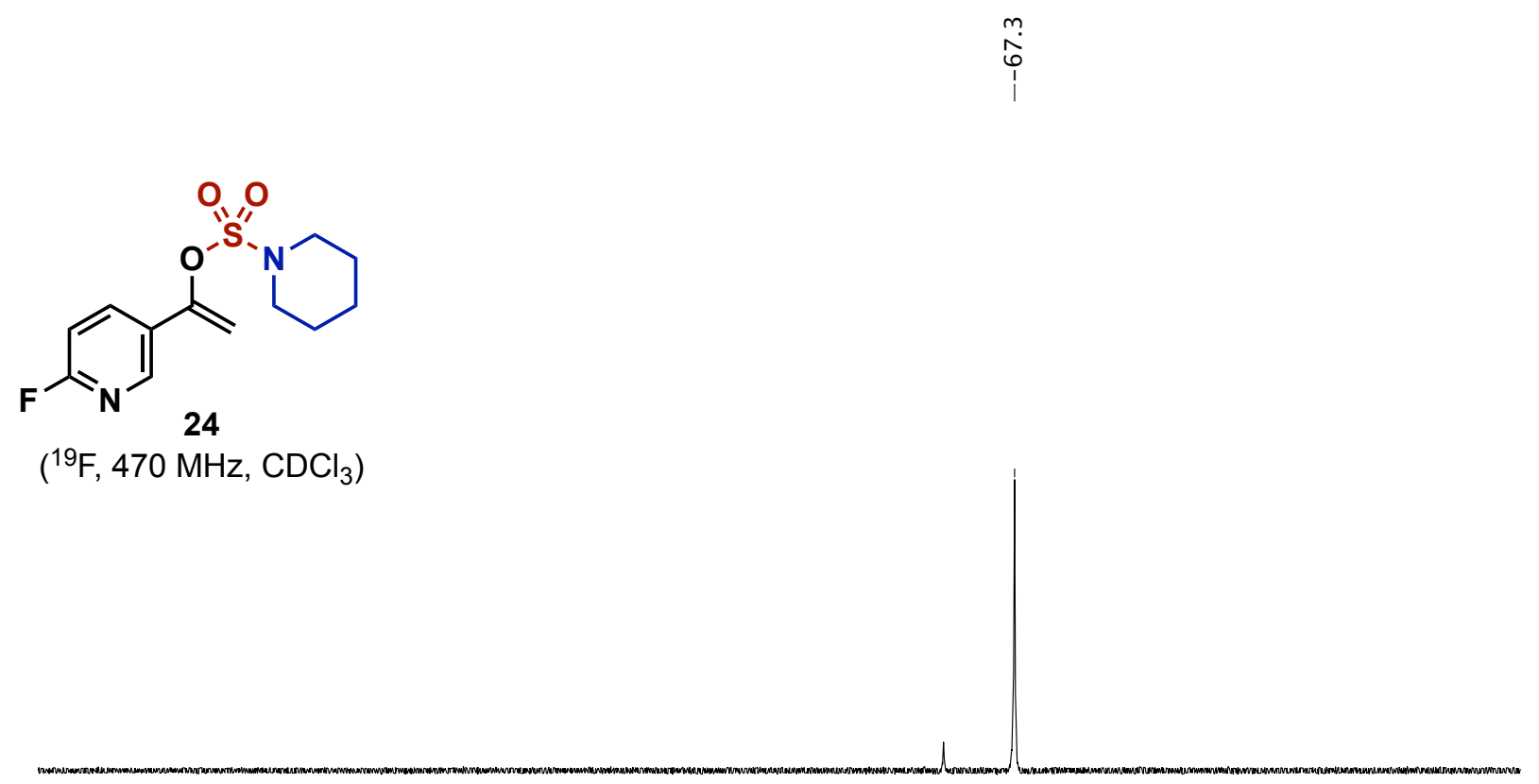

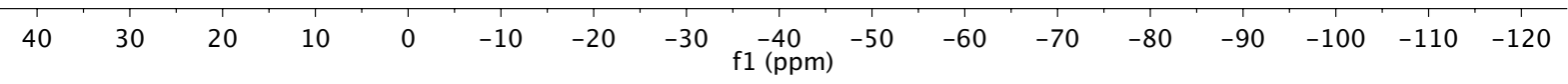



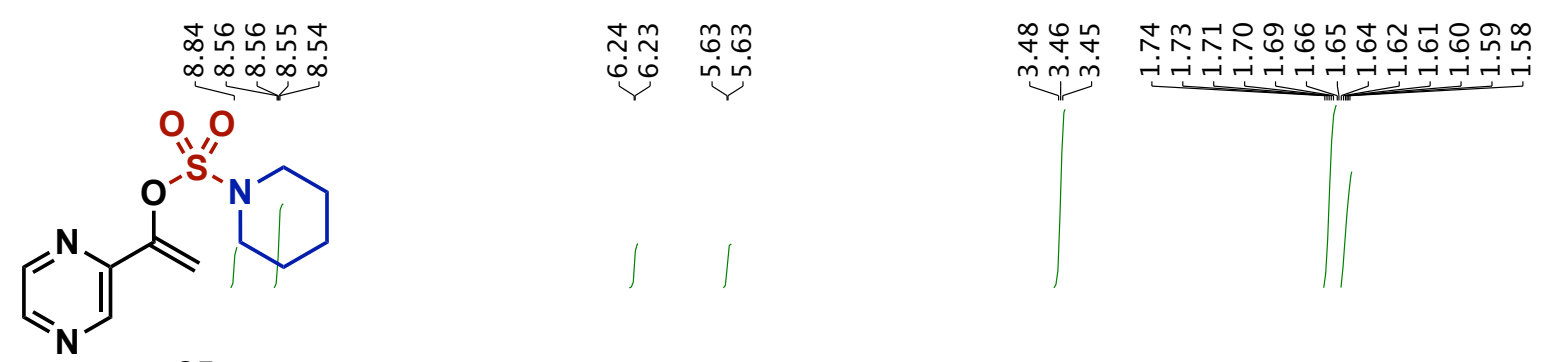

25

$\left({ }^{1} \mathrm{H}, 400 \mathrm{MHz}, \mathrm{CDCl}_{3}\right)$
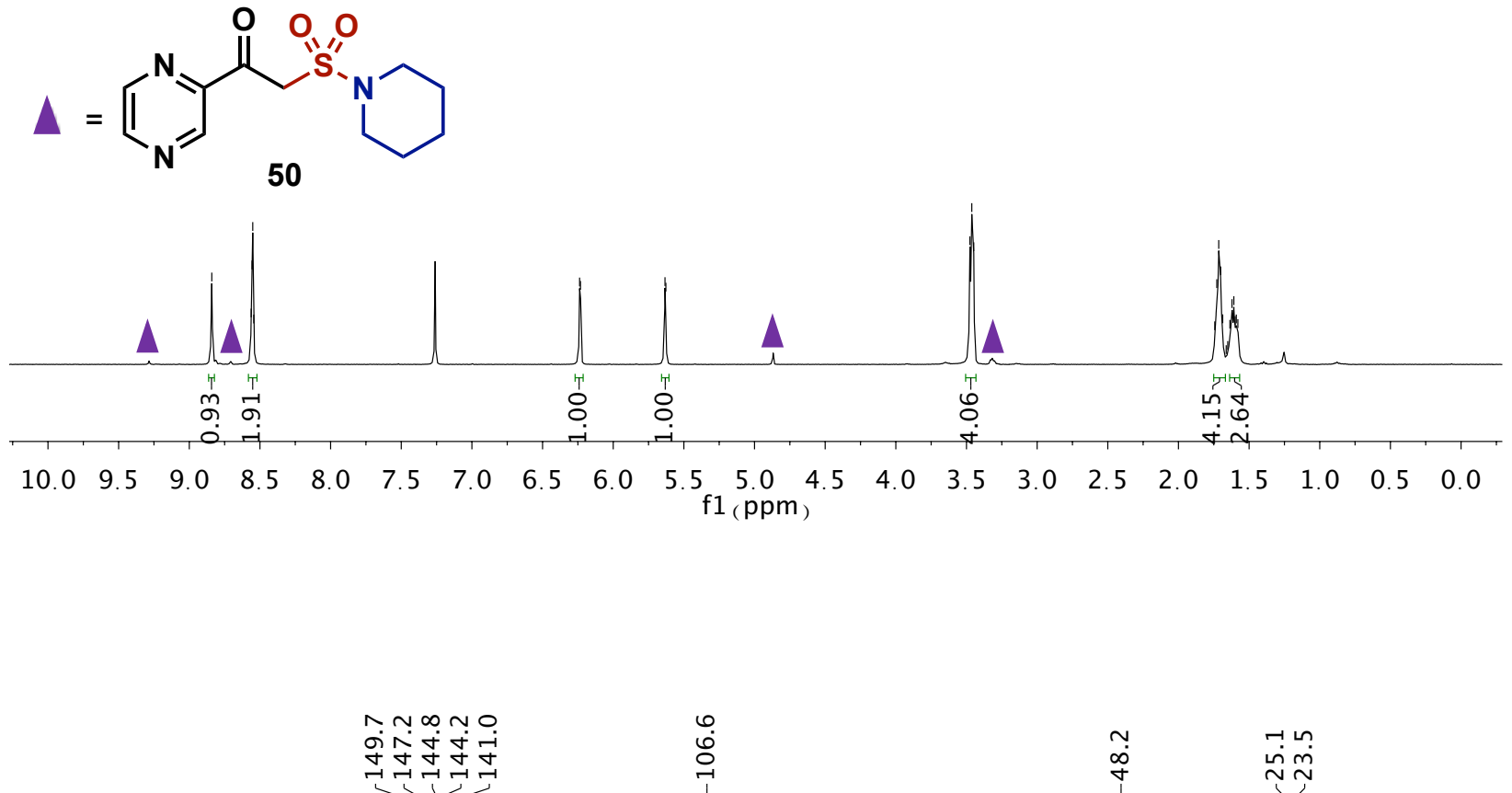

0
0
0
+1

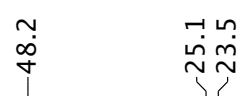<smiles>C=C(OS(=O)(=O)N1CCCCC1)c1cnccn1</smiles>

25

$\left({ }^{13} \mathrm{C}, 126 \mathrm{MHz}, \mathrm{CDCl}_{3}\right)$
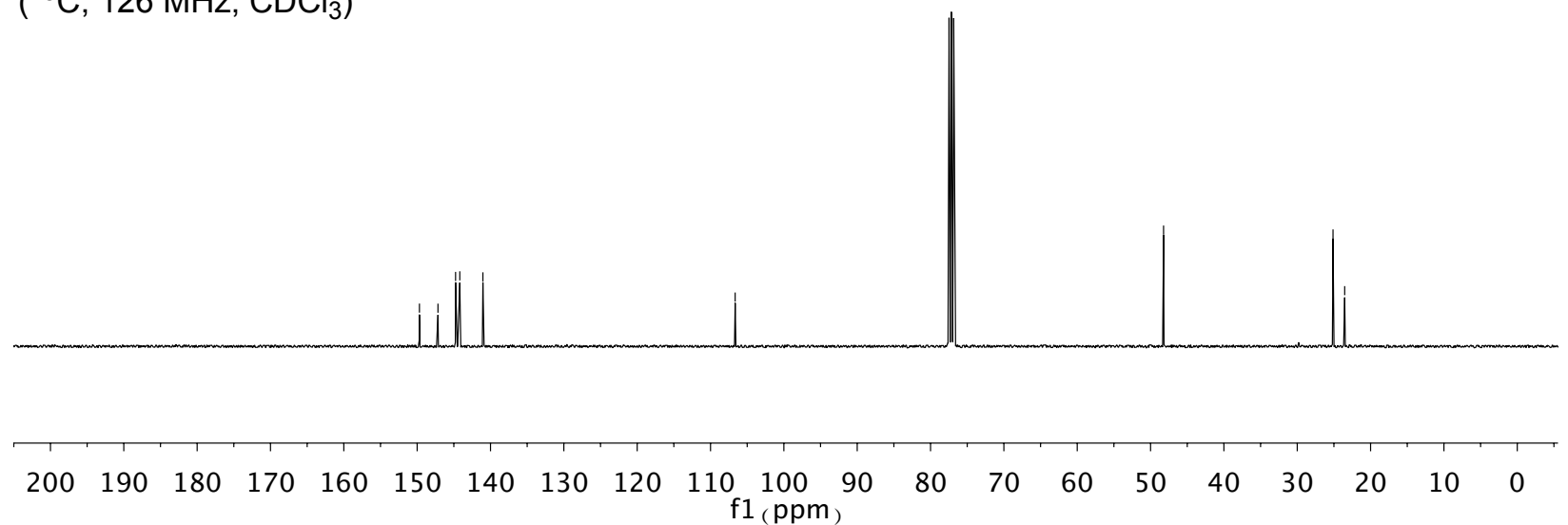


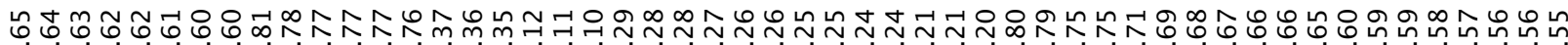
ம்<smiles>CC1=CCC(C(C)=C[Te])C=C1OS(=O)(=O)N1CCCCC1</smiles>

$\left({ }^{1} \mathrm{H}, 400 \mathrm{MHz}, \mathrm{CDCl}_{3}\right)$
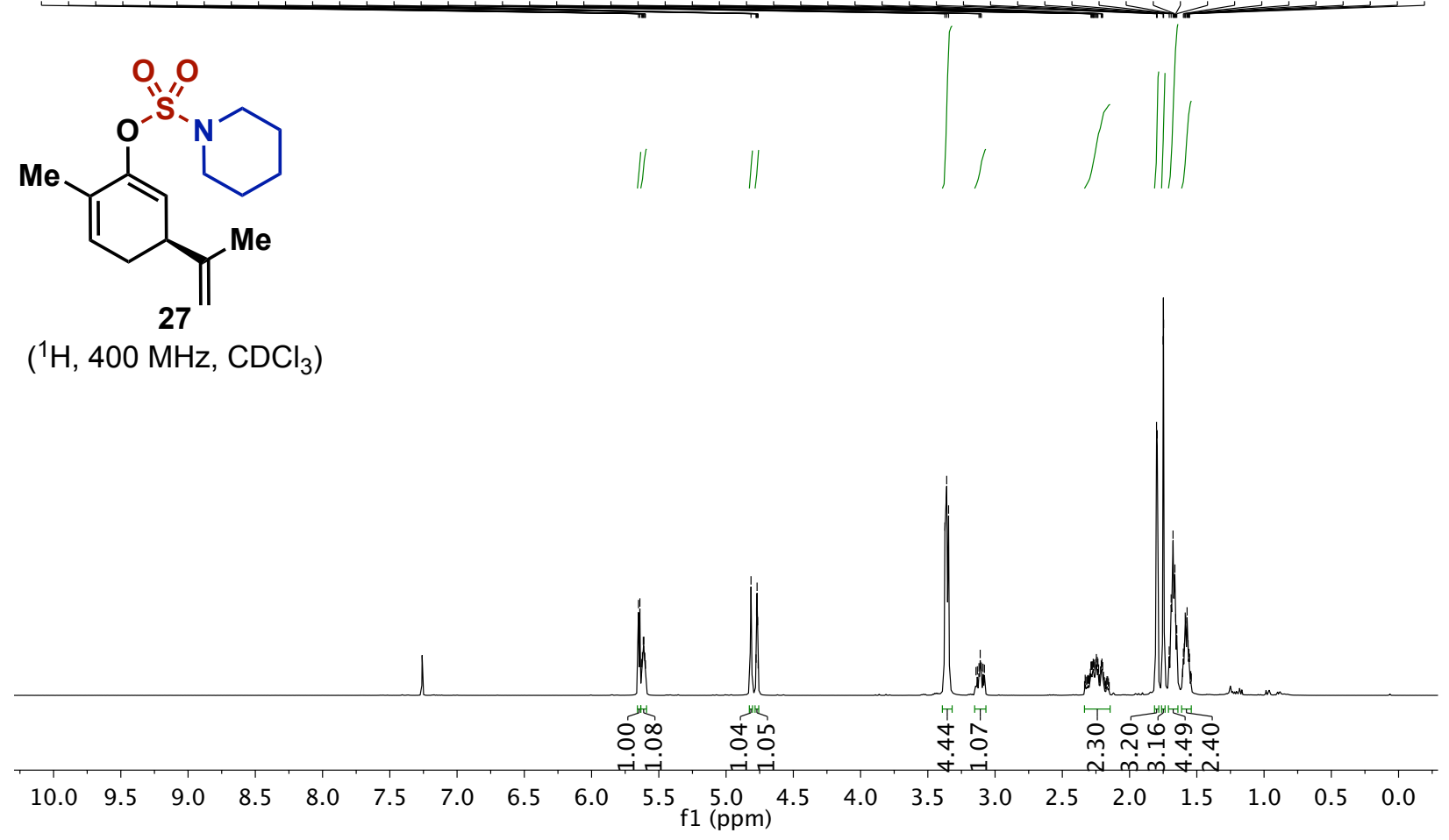

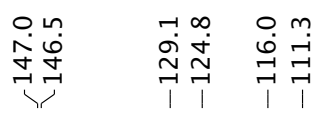

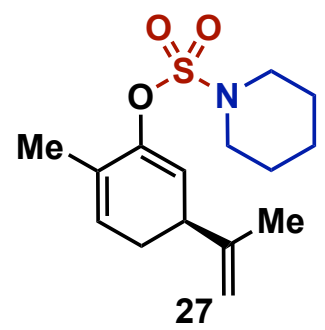

$\left({ }^{13} \mathrm{C}, 126 \mathrm{MHz}, \mathrm{CDCl}_{3}\right)$
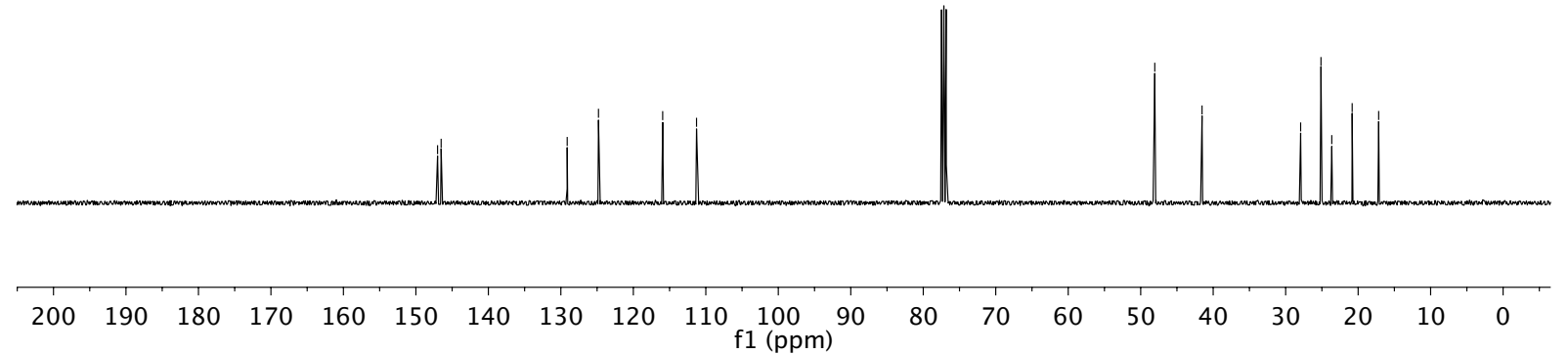


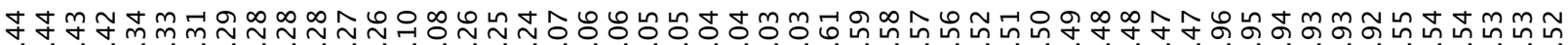

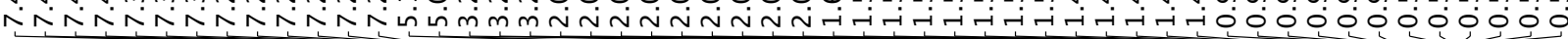<smiles>O=S1(=O)OC(c2ccccc2)=CC2CC23CCCCN31</smiles>

53

$\left({ }^{1} \mathrm{H}, 400 \mathrm{MHz}, \mathrm{CDCl}_{3}\right)$

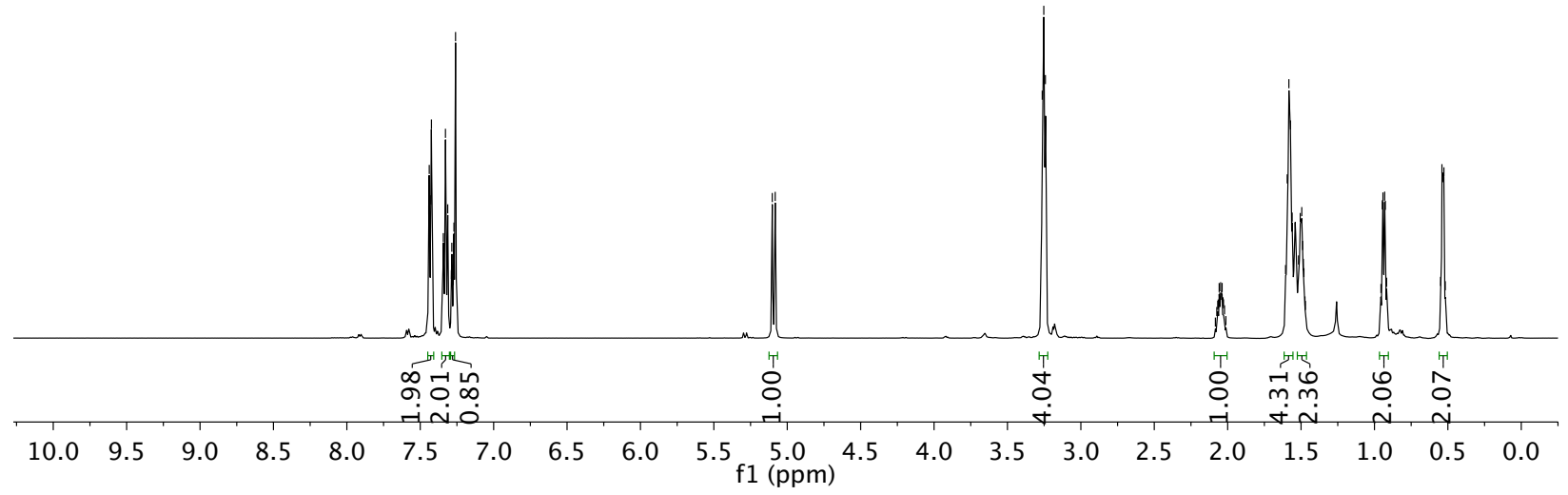

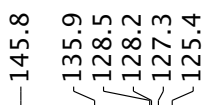<smiles>O=S1(=O)OC(c2ccccc2)=CC2CC23CCCCN31</smiles>

53

$\left({ }^{13} \mathrm{C}, 126 \mathrm{MHz}, \mathrm{CDCl}_{3}\right)$

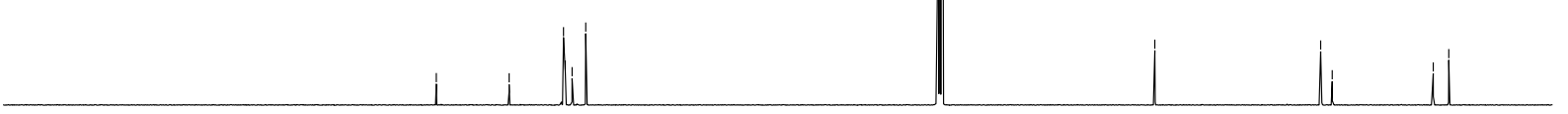

$\begin{array}{llllllllllllllllllll}200 & 190 & 180 & 170 & 160 & 150 & 140 & 130 & 120 & 110_{\mathrm{f} 1(\mathrm{ppm})}^{100} 90 & 80 & 70 & 60 & 50 & 40 & 30 & 20 & 10 & 0\end{array}$



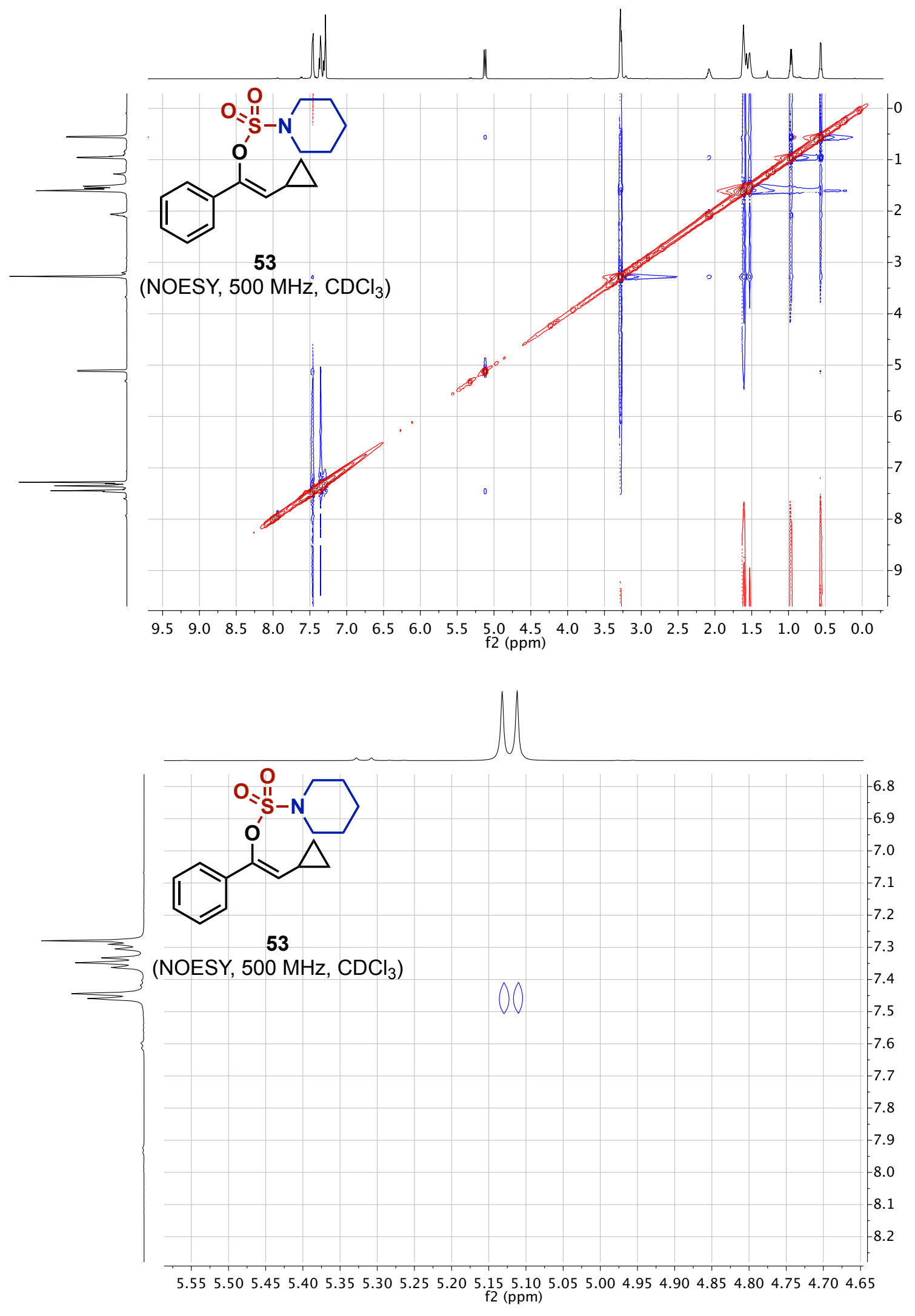


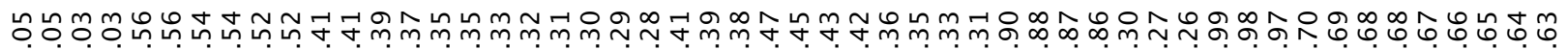

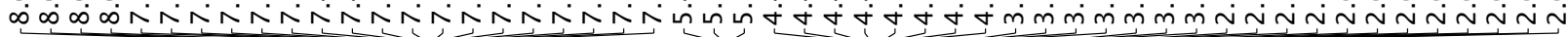<smiles>O=C1c2ccccc2CCC1S(=O)(=O)NCC1=IC=CC=C1</smiles>

29

$\left({ }^{1} \mathrm{H}, 400 \mathrm{MHz}, \mathrm{CDCl}_{3}\right)$
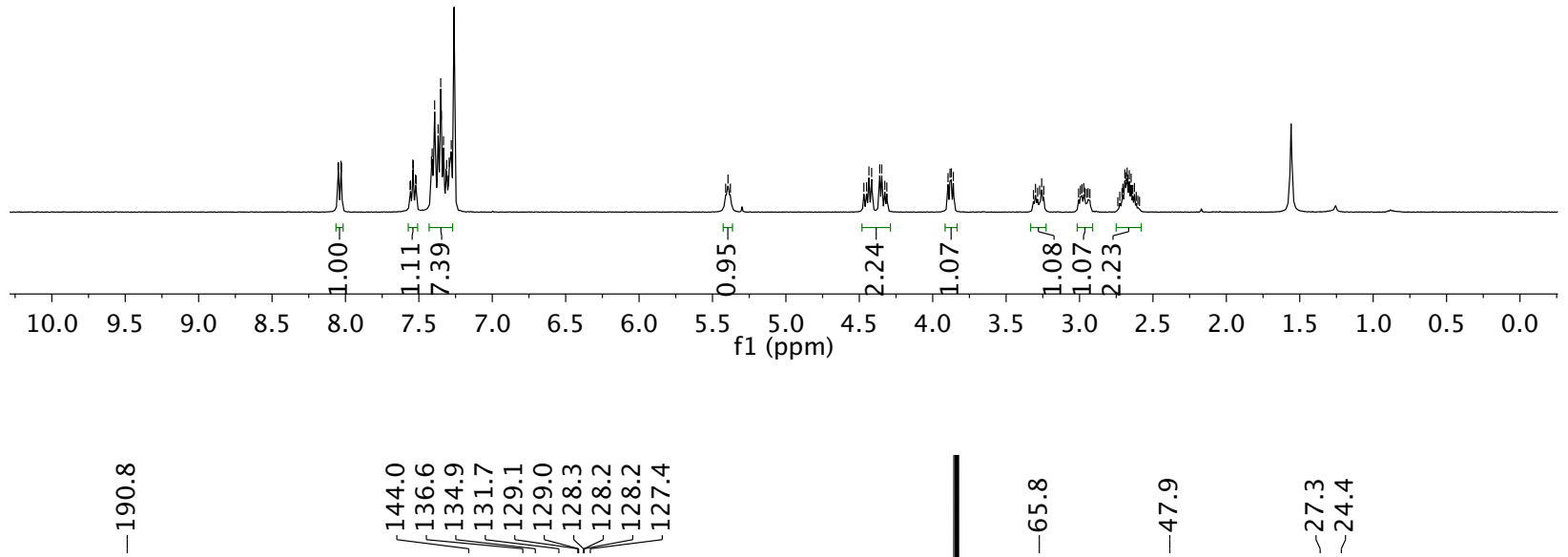

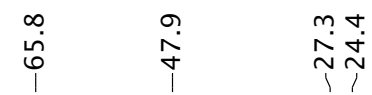<smiles>O=C1c2ccccc2CCC1S(=O)(=O)NCc1ccccc1</smiles>

29

$\left({ }^{13} \mathrm{C}, 126 \mathrm{MHz}, \mathrm{CDCl}_{3}\right)$

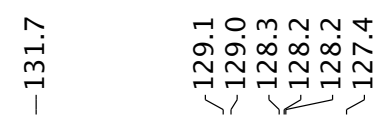
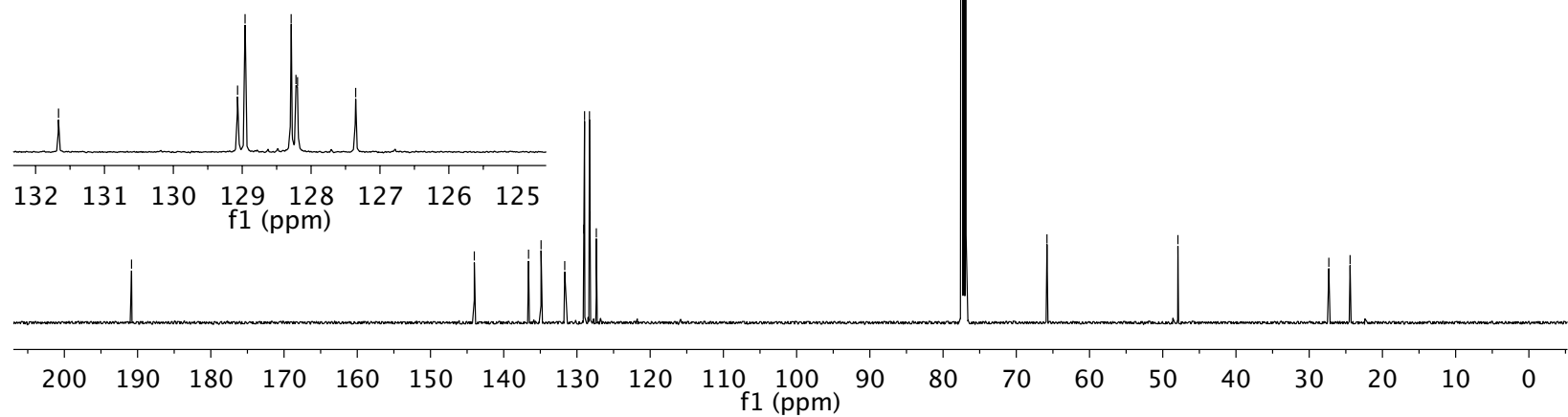


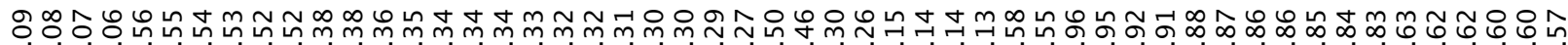

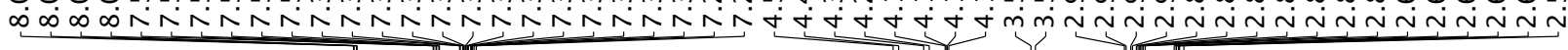<smiles>CN(Cc1ccccc1)S(=O)(=O)C1CCc2ccccc2C1=O</smiles>

$\left({ }^{1} \mathrm{H}, 400 \mathrm{MHz}, \mathrm{CDCl}_{3}\right)$

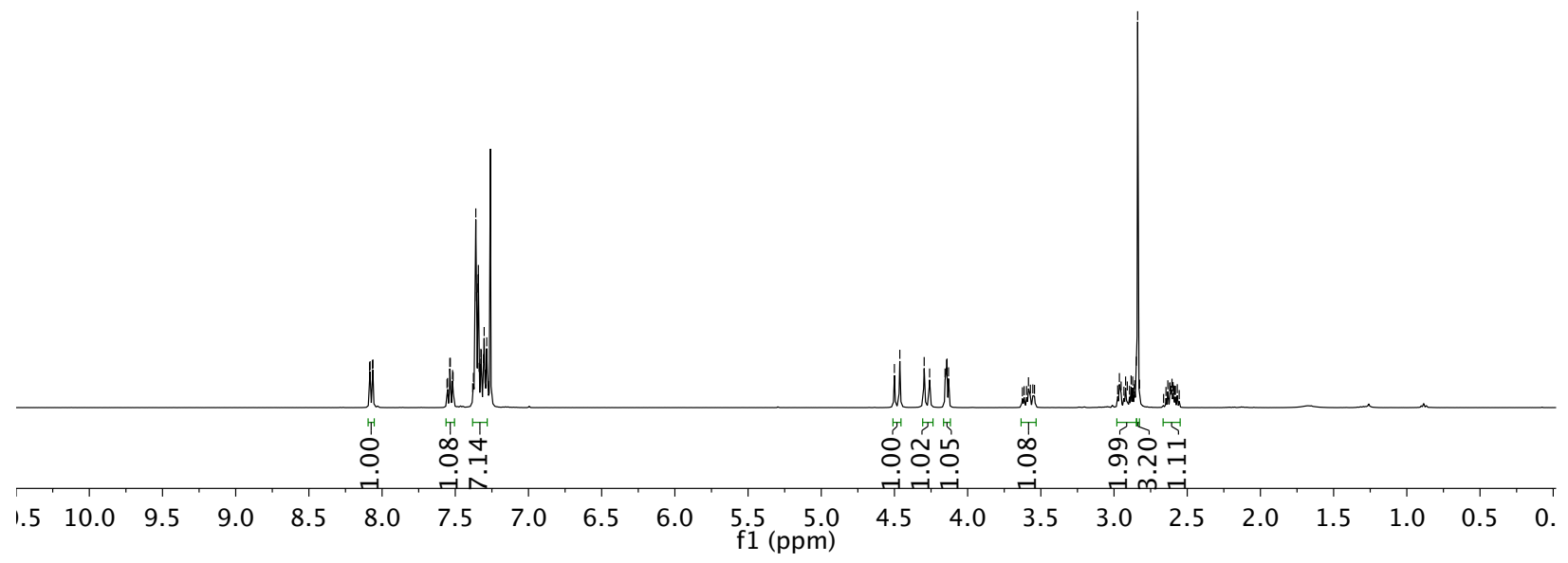

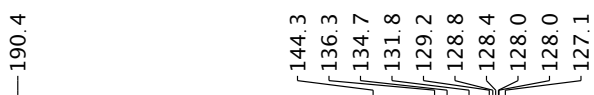

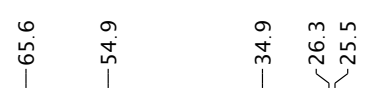<smiles>CN(Cc1ccccc1)S(=O)(=O)C1CCc2ccccc2C1=O</smiles>

30

$\left({ }^{13} \mathrm{C}, 126 \mathrm{MHz}, \mathrm{CDCl}_{3}\right)$

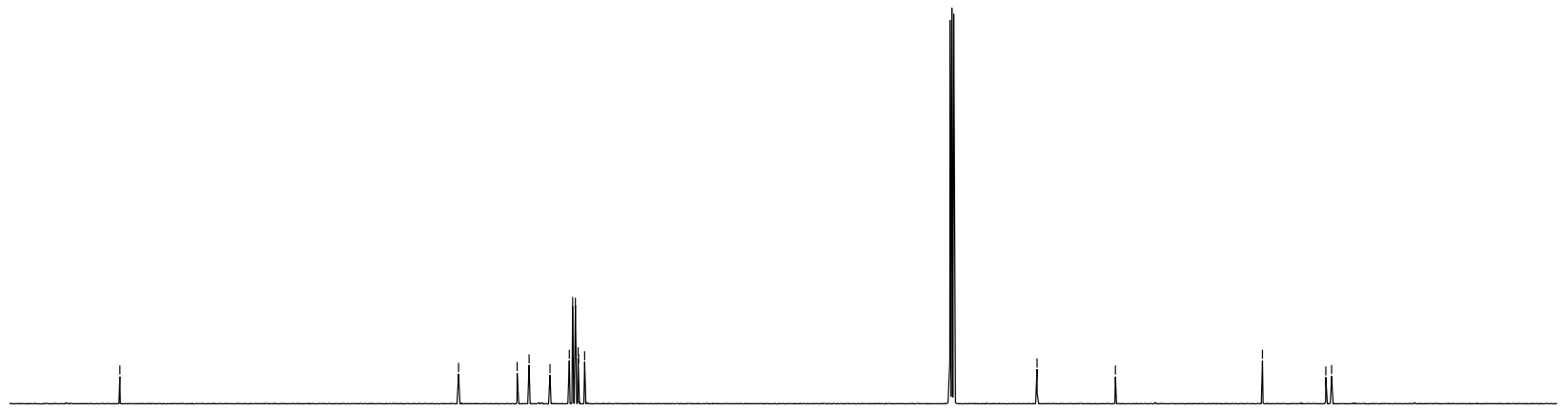

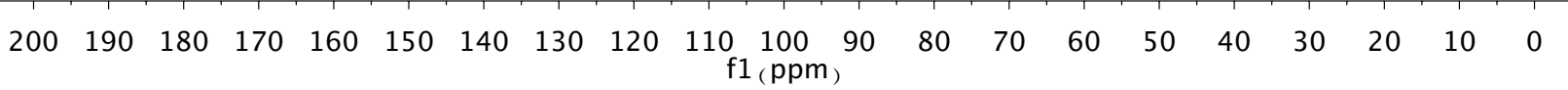




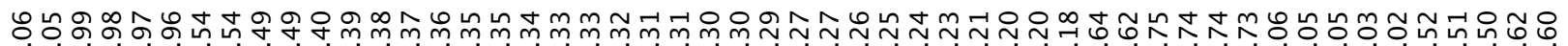

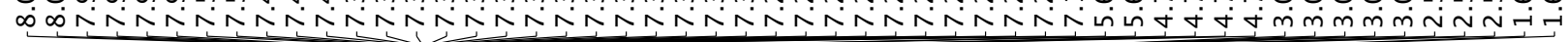<smiles>C[C@H](NS(=O)(=O)C1CCc2ccccc2C1=O)c1ccccc1</smiles>

31

$\left({ }^{1} \mathrm{H}, 400 \mathrm{MHz}, \mathrm{CDCl}_{3}\right)$

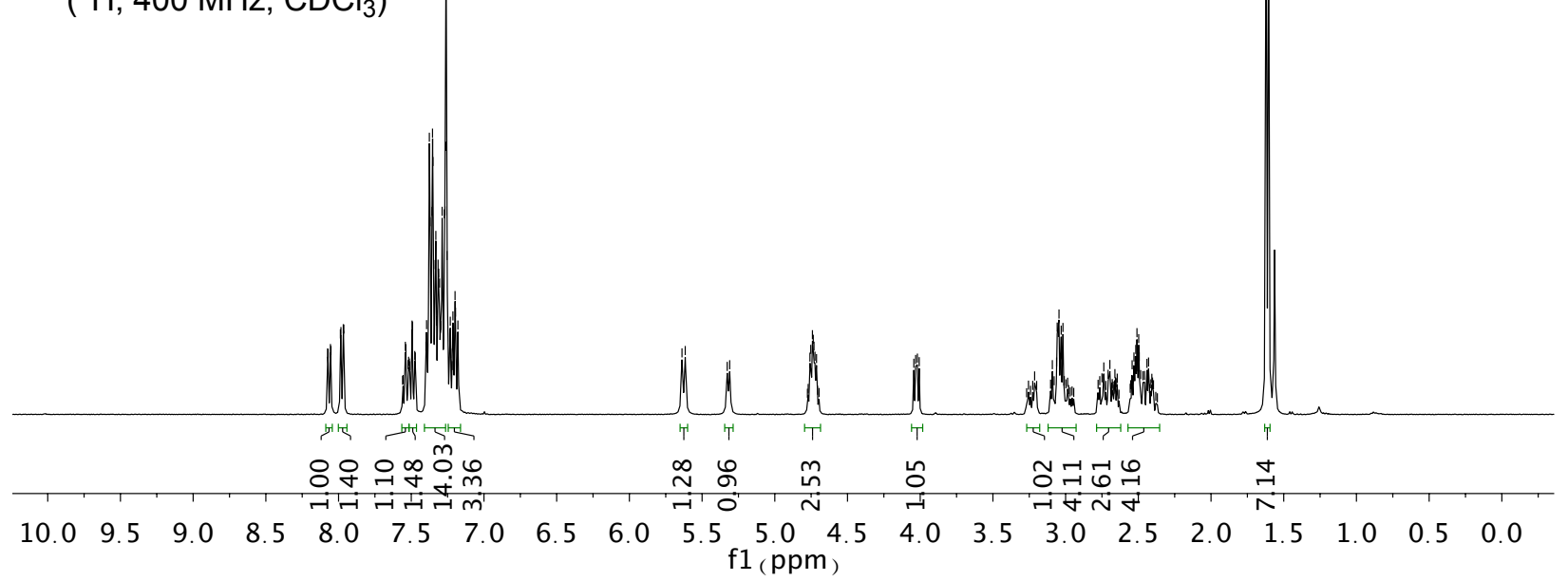

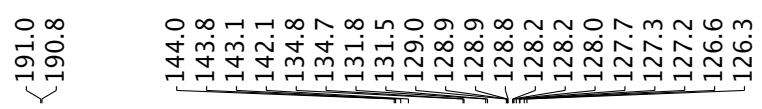

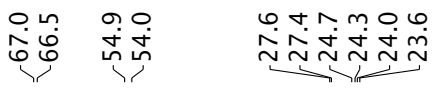<smiles>C[C@@H](NS(=O)(=O)C1CCc2ccccc2C1=O)c1ccccc1</smiles>

31

$\left({ }^{13} \mathrm{C}, 126 \mathrm{MHz}, \mathrm{CDCl}_{3}\right)$

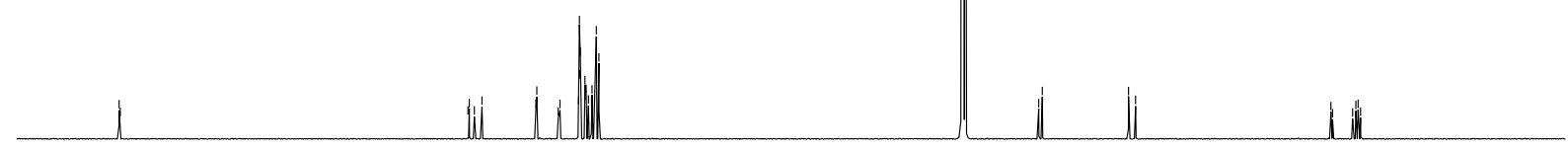

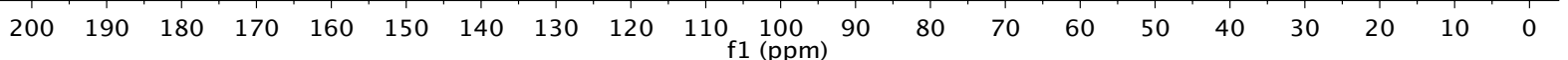




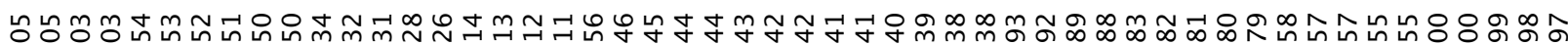

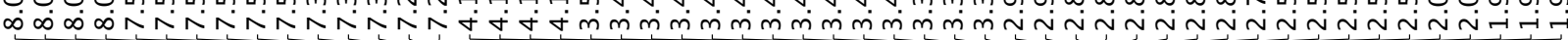<smiles>O=C1c2ccccc2CCC1S(=O)(=O)N1CCCC1</smiles>

28

$\left({ }^{1} \mathrm{H}, 400 \mathrm{MHz}, \mathrm{CDCl}_{3}\right)$
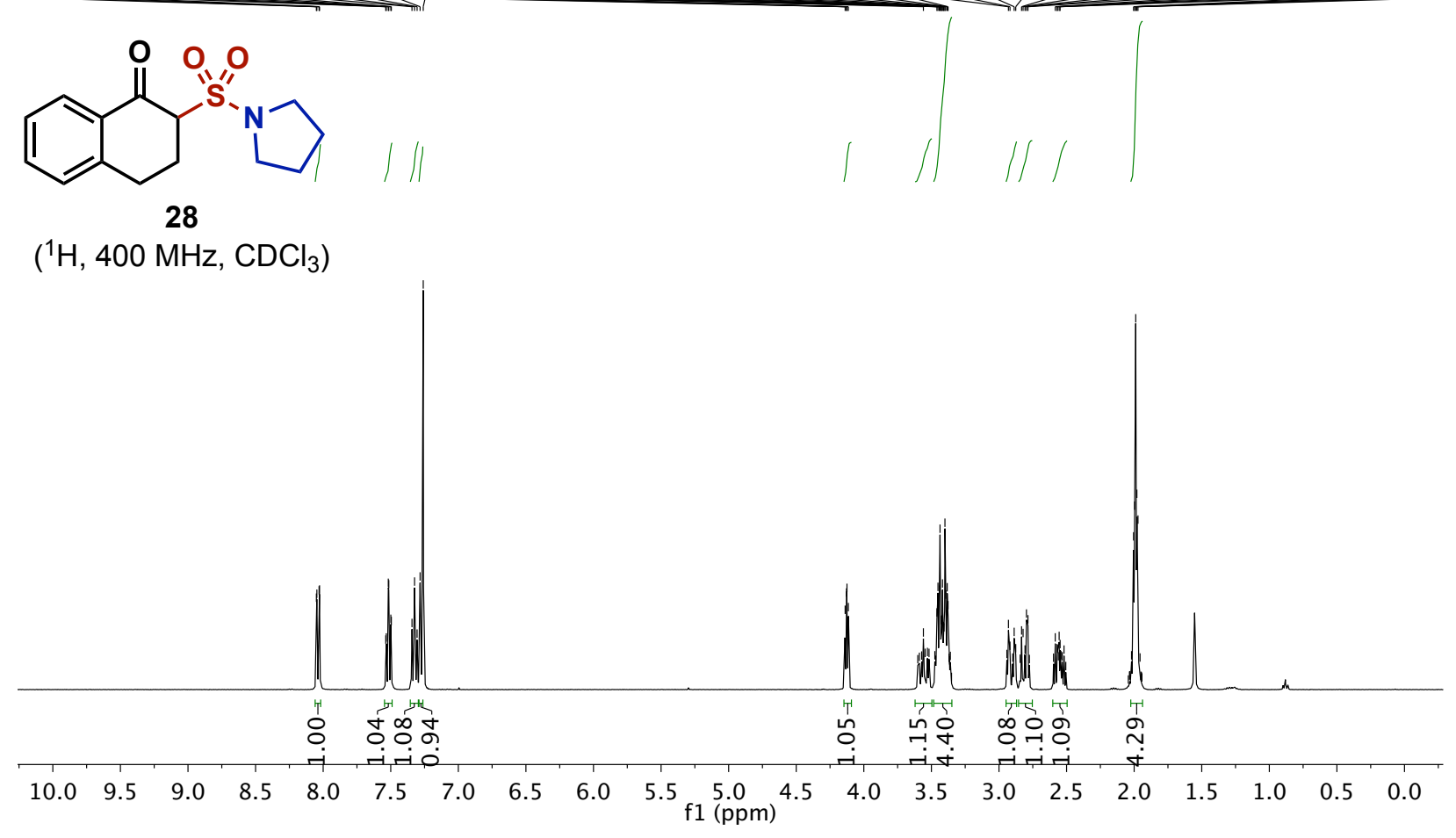

促

\section{$0.09 .5 \quad 9.0 \quad 8.5 \quad 8.0 \quad 7.5$}

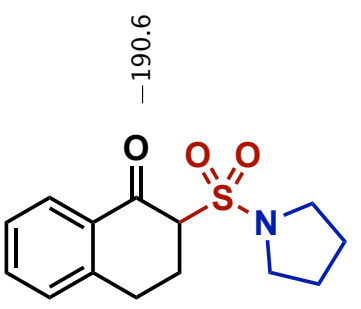

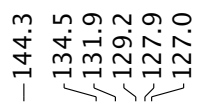

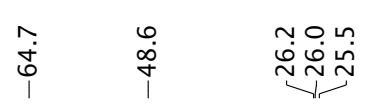

$\left({ }^{13} \mathrm{C}, 126 \mathrm{MHz}, \mathrm{CDCl}_{3}\right)$
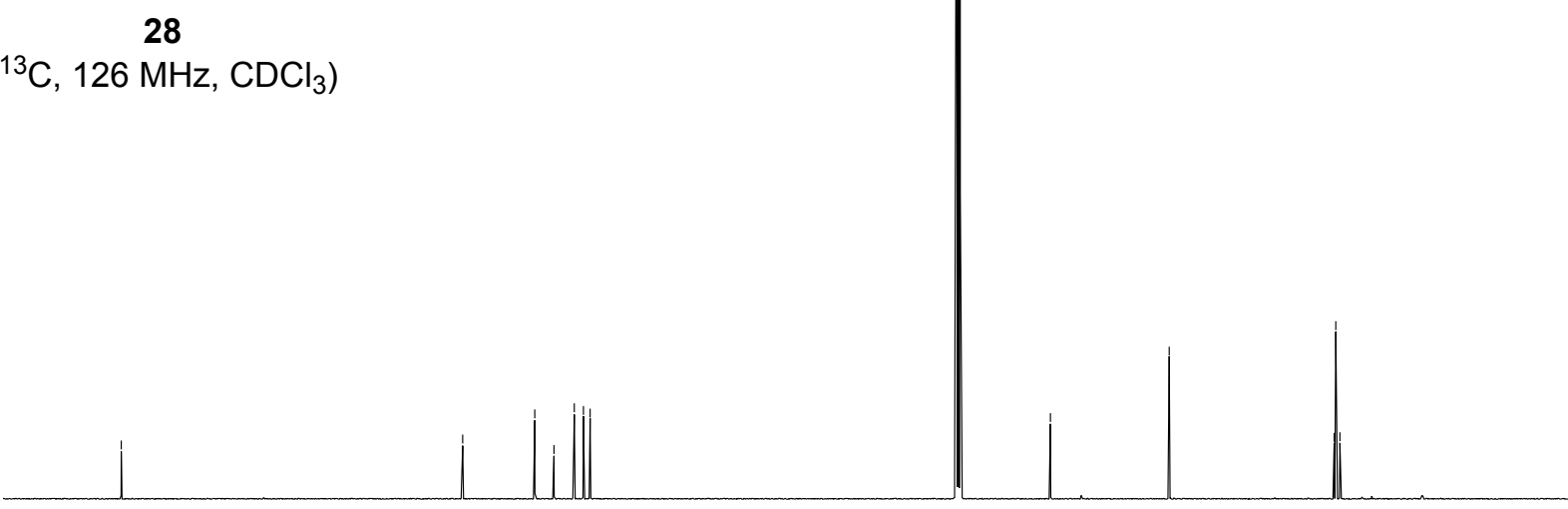

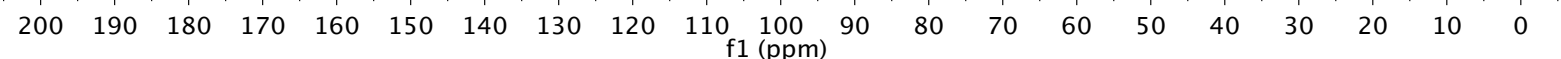




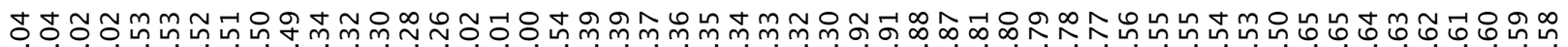

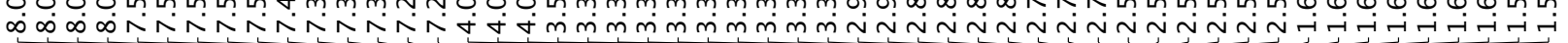<smiles>O=C1c2ccccc2CCC1S(=O)(=O)N1CCCCC1</smiles>

32

$\left({ }^{1} \mathrm{H}, 400 \mathrm{MHz}, \mathrm{CDCl}_{3}\right)$

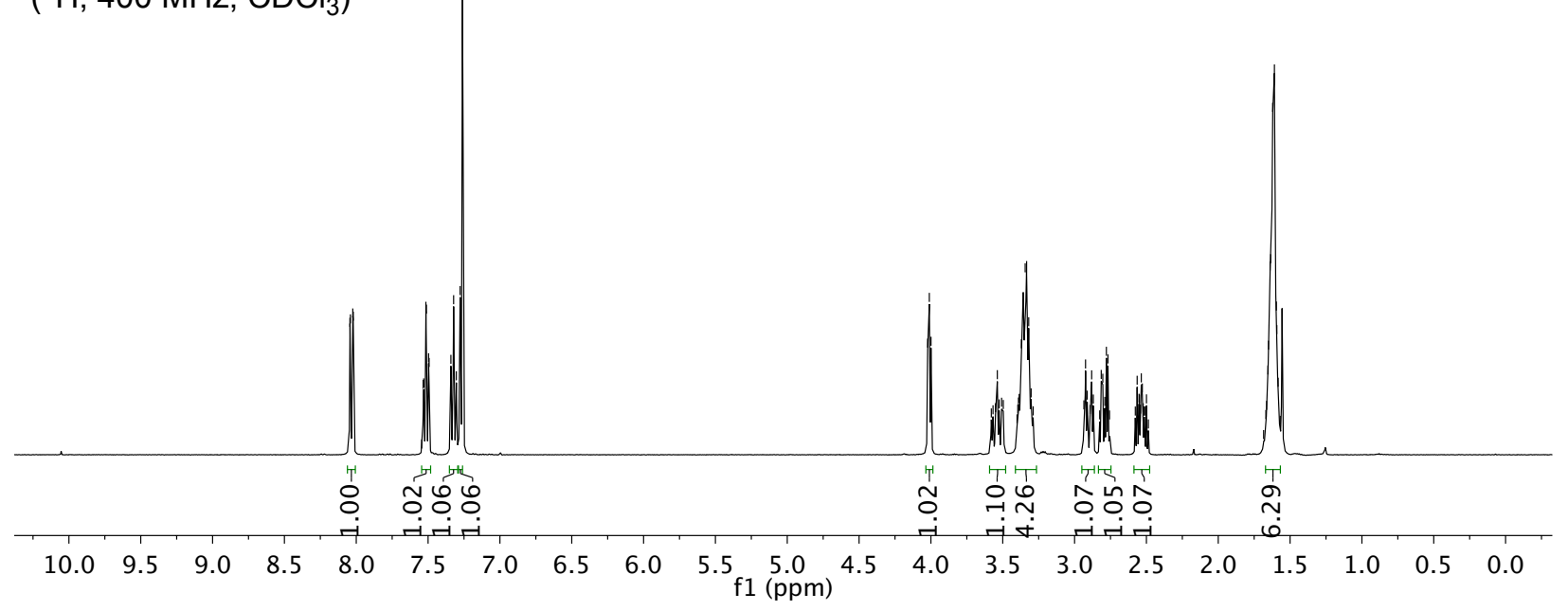

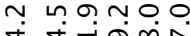

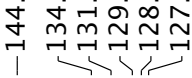<smiles>O=C1c2ccccc2CCC1S(=O)(=O)N1CCCCC1</smiles>

32

$\left({ }^{13} \mathrm{C}, 126 \mathrm{MHz}, \mathrm{CDCl}_{3}\right)$
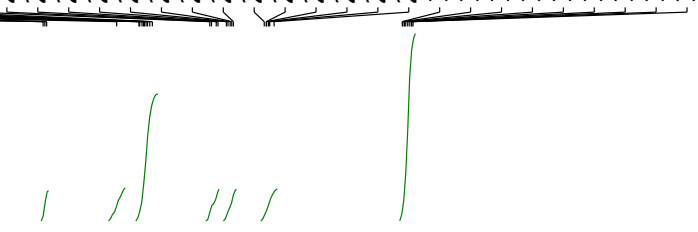


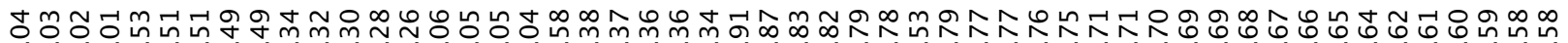

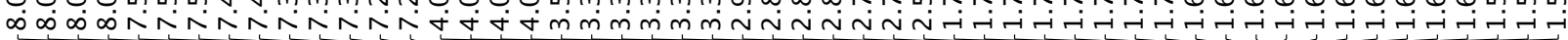<smiles>O=C1c2ccccc2CCC1S(=O)(=O)N1CCC=CCC1</smiles>

33

$\left({ }^{1} \mathrm{H}, 400 \mathrm{MHz}, \mathrm{CDCl}_{3}\right)$

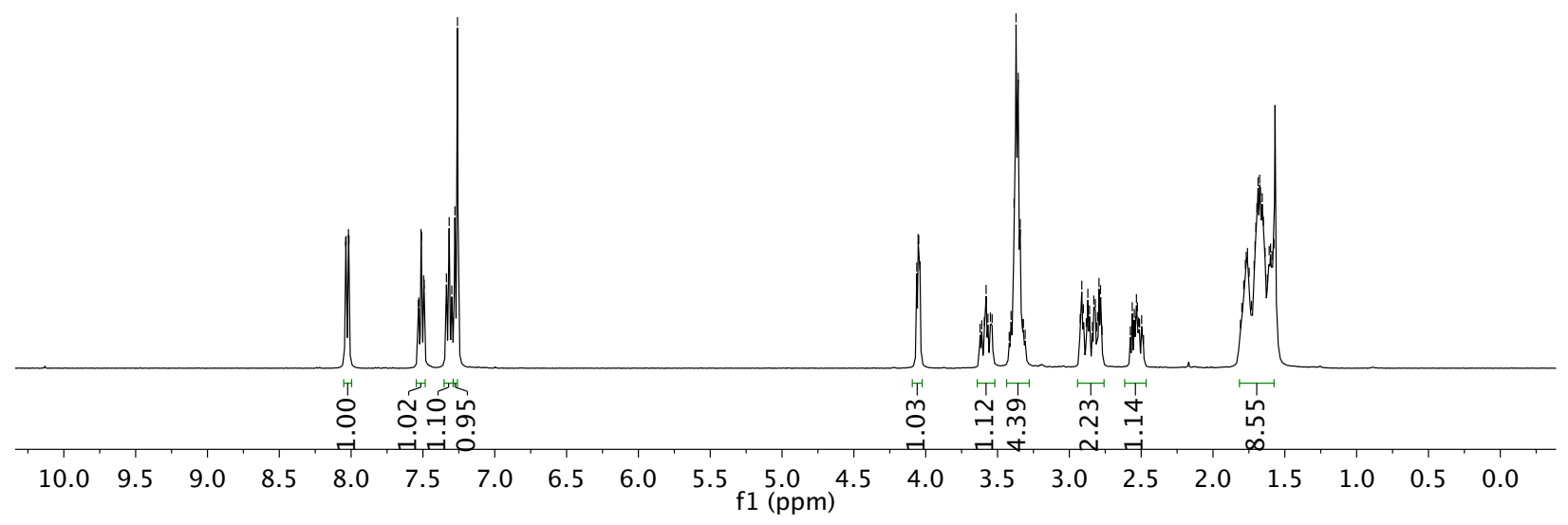<smiles>O=C1c2ccccc2CCC1S(=O)(=O)N1CCCCCC1</smiles>

33

$\left({ }^{13} \mathrm{C}, 126 \mathrm{MHz}, \mathrm{CDCl}_{3}\right)$
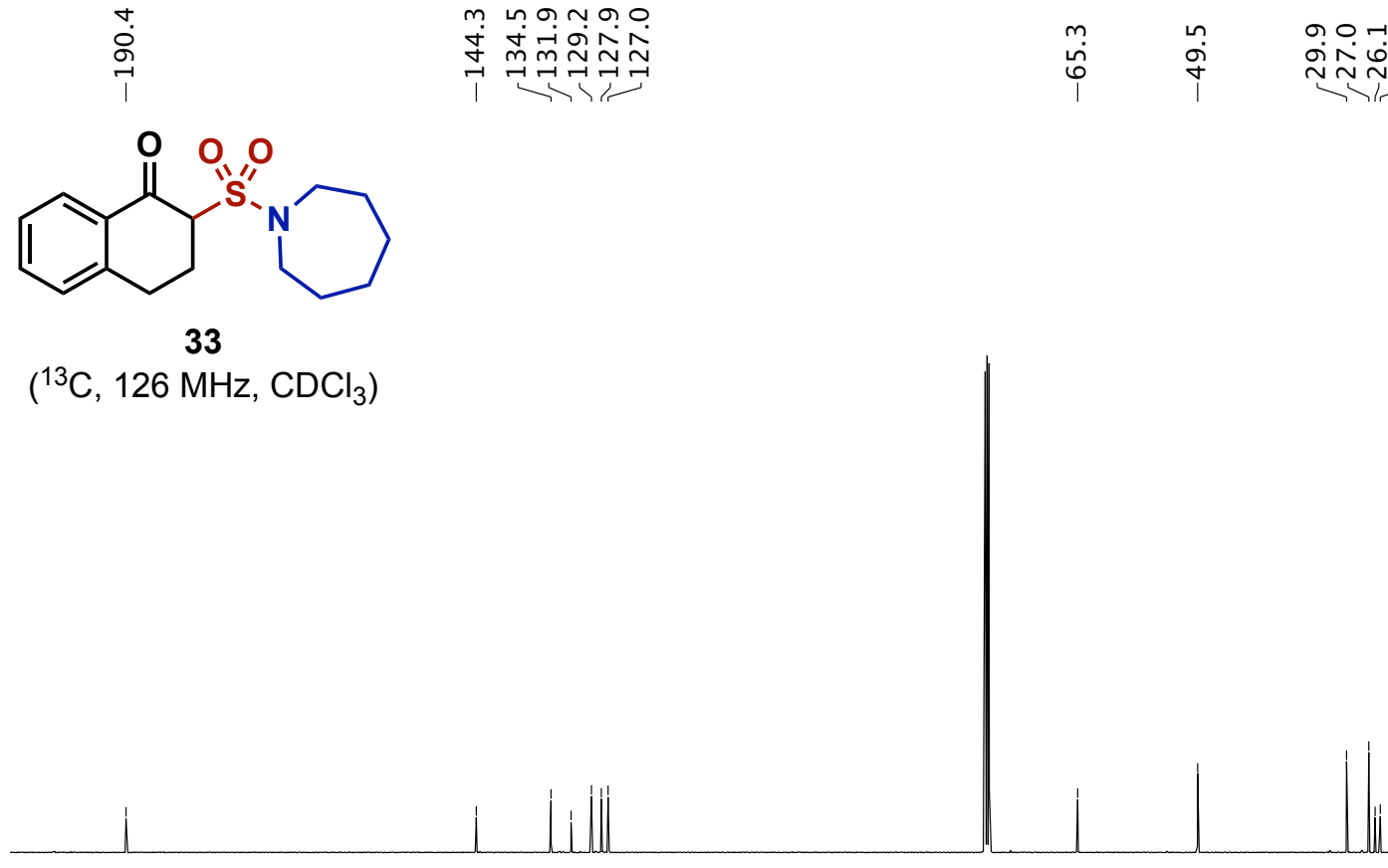

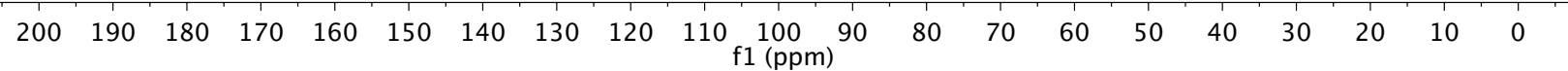




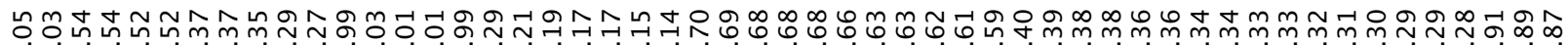

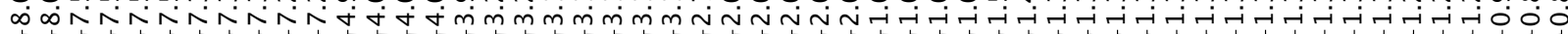<smiles>O=C1c2ccccc2CCC1S(=O)(=O)NCCO</smiles>

$\left({ }^{1} \mathrm{H}, 400 \mathrm{MHz}, \mathrm{CDCl}_{3}\right)$

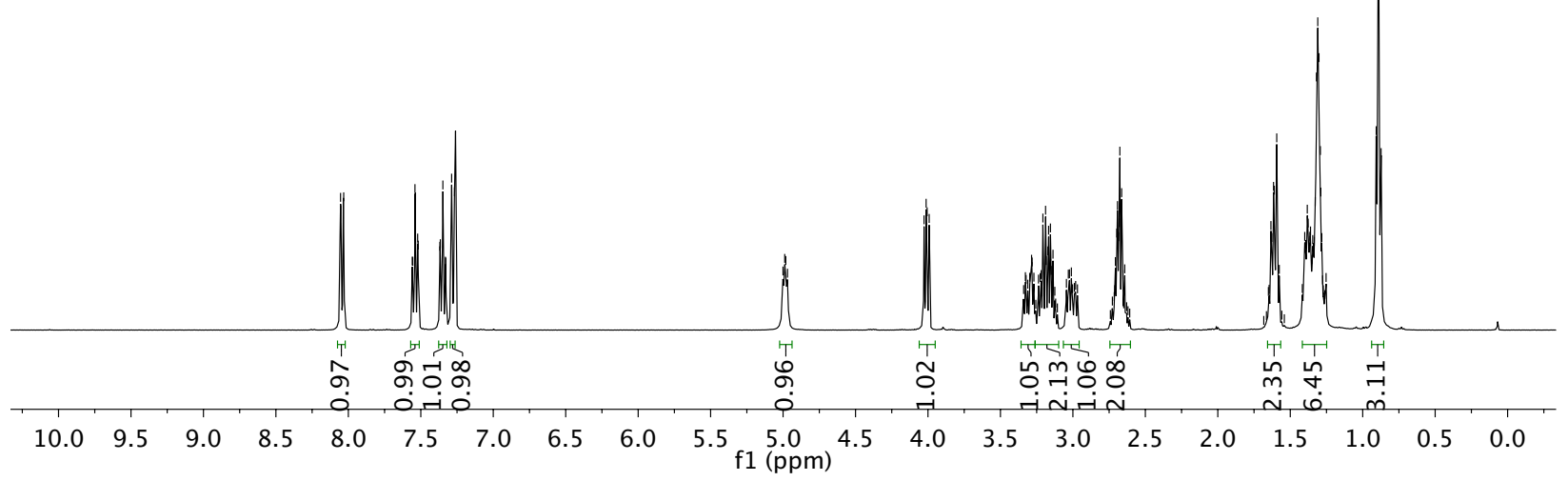<smiles>O=C1c2ccccc2CCC1S(=O)(=O)NCO</smiles>

$\left({ }^{13} \mathrm{C}, 126 \mathrm{MHz}, \mathrm{CDCl}_{3}\right)$

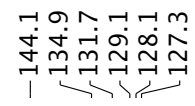

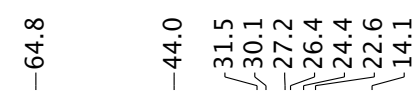

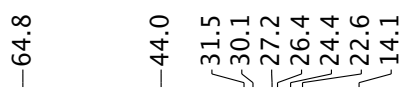

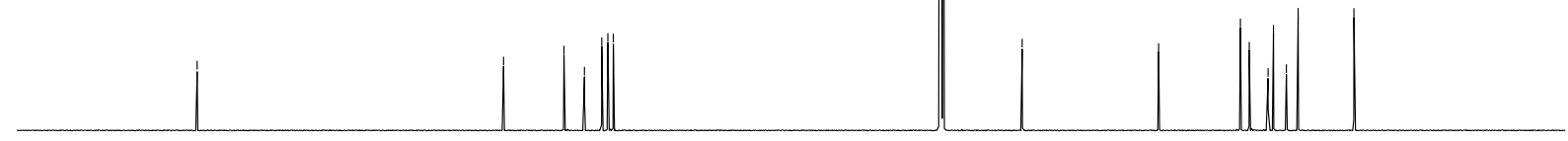

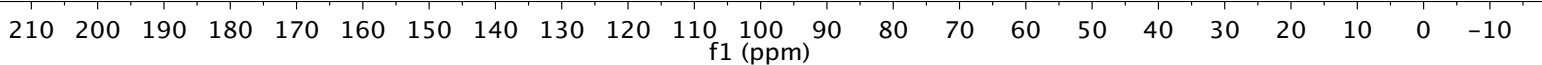




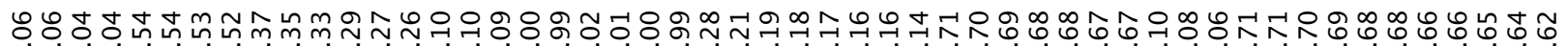

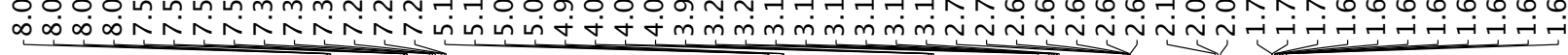<smiles>CC(C)=CCCCNS(=O)(=O)C1CCc2ccccc2C1=O</smiles>

35

$\left({ }^{1} \mathrm{H}, 400 \mathrm{MHz}, \mathrm{CDCl}_{3}\right)$

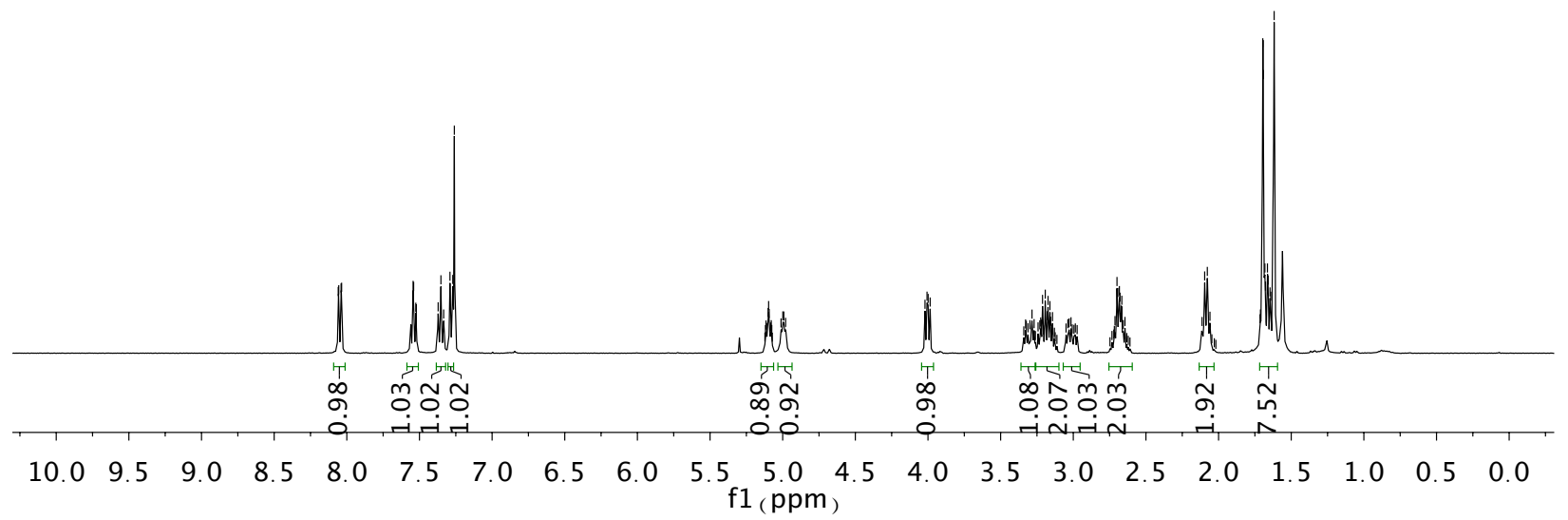<smiles>CC(C)=CCCCNS(=O)(=O)C1CCc2ccccc2C1=O</smiles>

35

$\left({ }^{13} \mathrm{C}, 126 \mathrm{MHz}, \mathrm{CDCl}_{3}\right)$

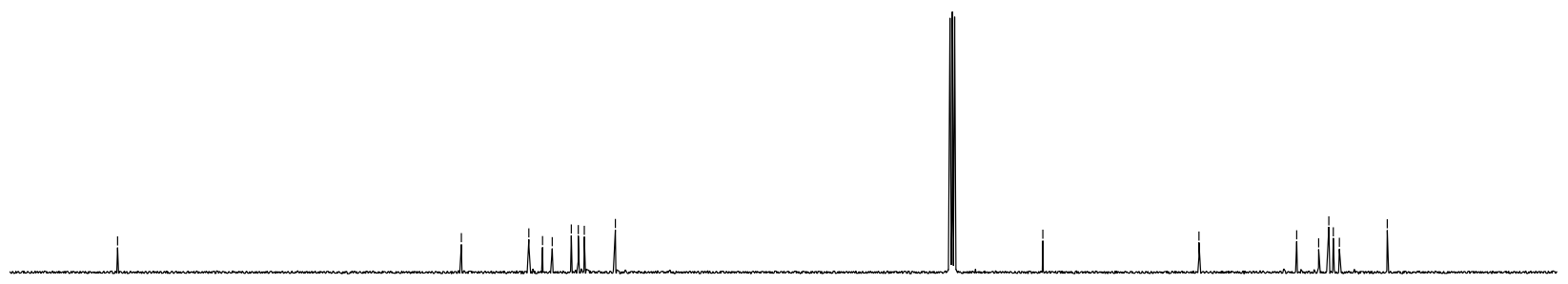

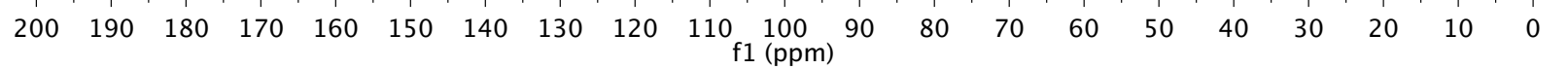




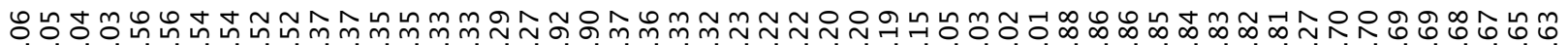

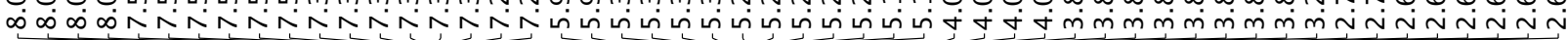<smiles>C=CCNS(=O)(=O)C1CCc2ccccc2C1=O</smiles>

36

$\left.\left({ }^{1} \mathrm{H}, 126 \mathrm{MHz}^{\mathrm{CDCl}}\right)_{3}\right)$

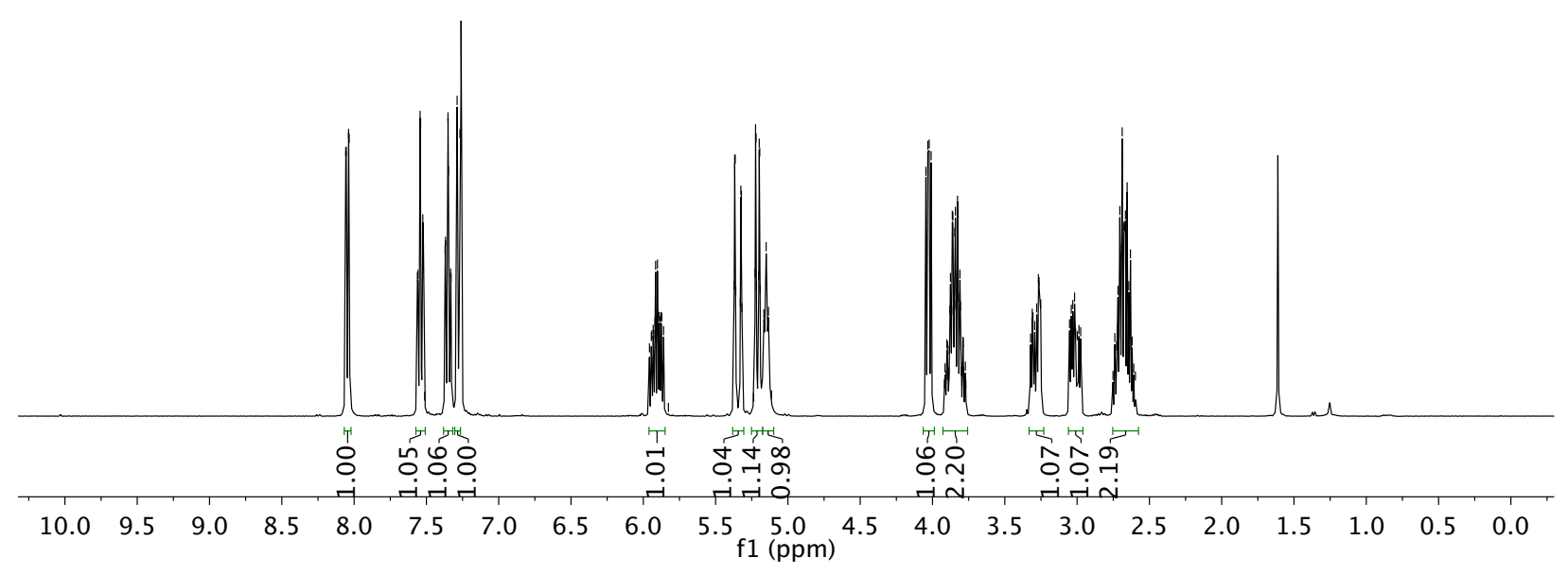

คำ

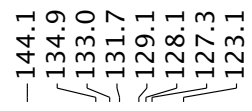

lo $\quad$ m $\quad$ m<smiles>C=CCNS(=O)(=O)C1CCc2ccccc2C1=O</smiles>

36

$\left({ }^{13} \mathrm{C}, 126 \mathrm{MHz}, \mathrm{CDCl}_{3}\right)$

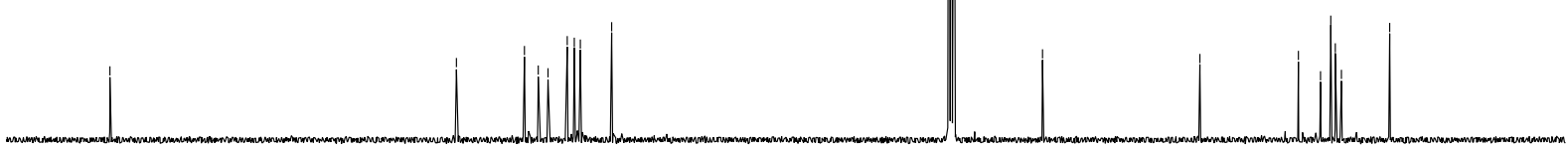

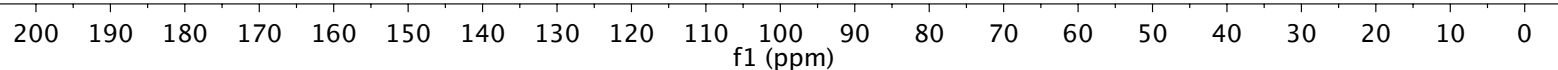




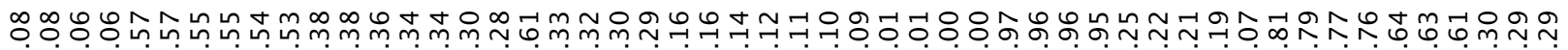
$\infty \infty \infty \infty N \hat{N} N \hat{N} N \hat{N} N \hat{N} N$ U<smiles>C#CCNS(=O)(=O)C1CCc2ccccc2C1=O</smiles>

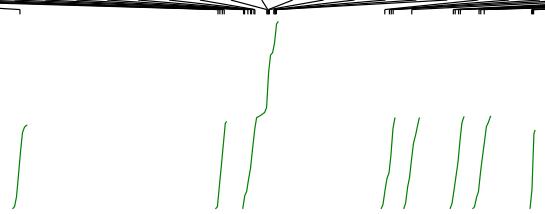

37

$\left({ }^{1} \mathrm{H}, 126 \mathrm{MHz}, \mathrm{CDCl}_{3}\right)$

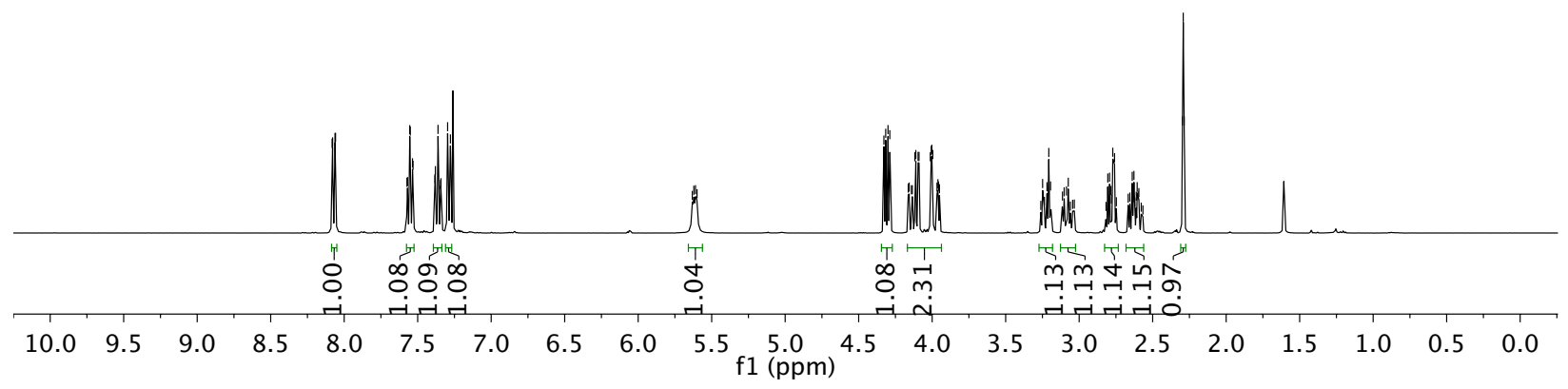

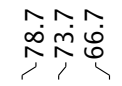

miñm

m்ì<smiles>C#CCNS(=O)(=O)C1CCc2ccccc2C1=O</smiles>

37

$\left({ }^{13} \mathrm{C}, 126 \mathrm{MHz}, \mathrm{CDCl}_{3}\right)$

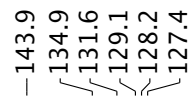




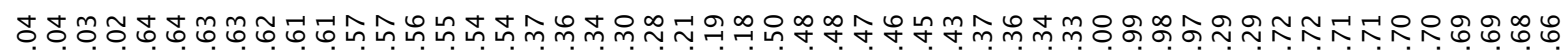

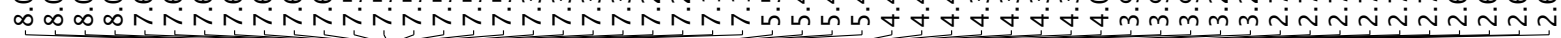

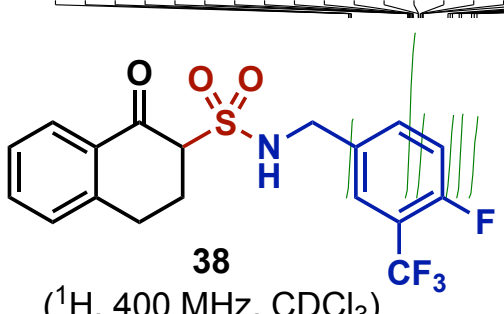

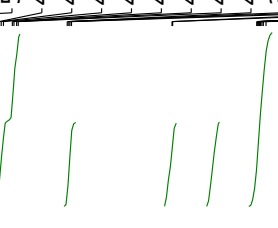

$\left({ }^{1} \mathrm{H}, 400 \mathrm{MHz}, \mathrm{CDCl}_{3}\right)$
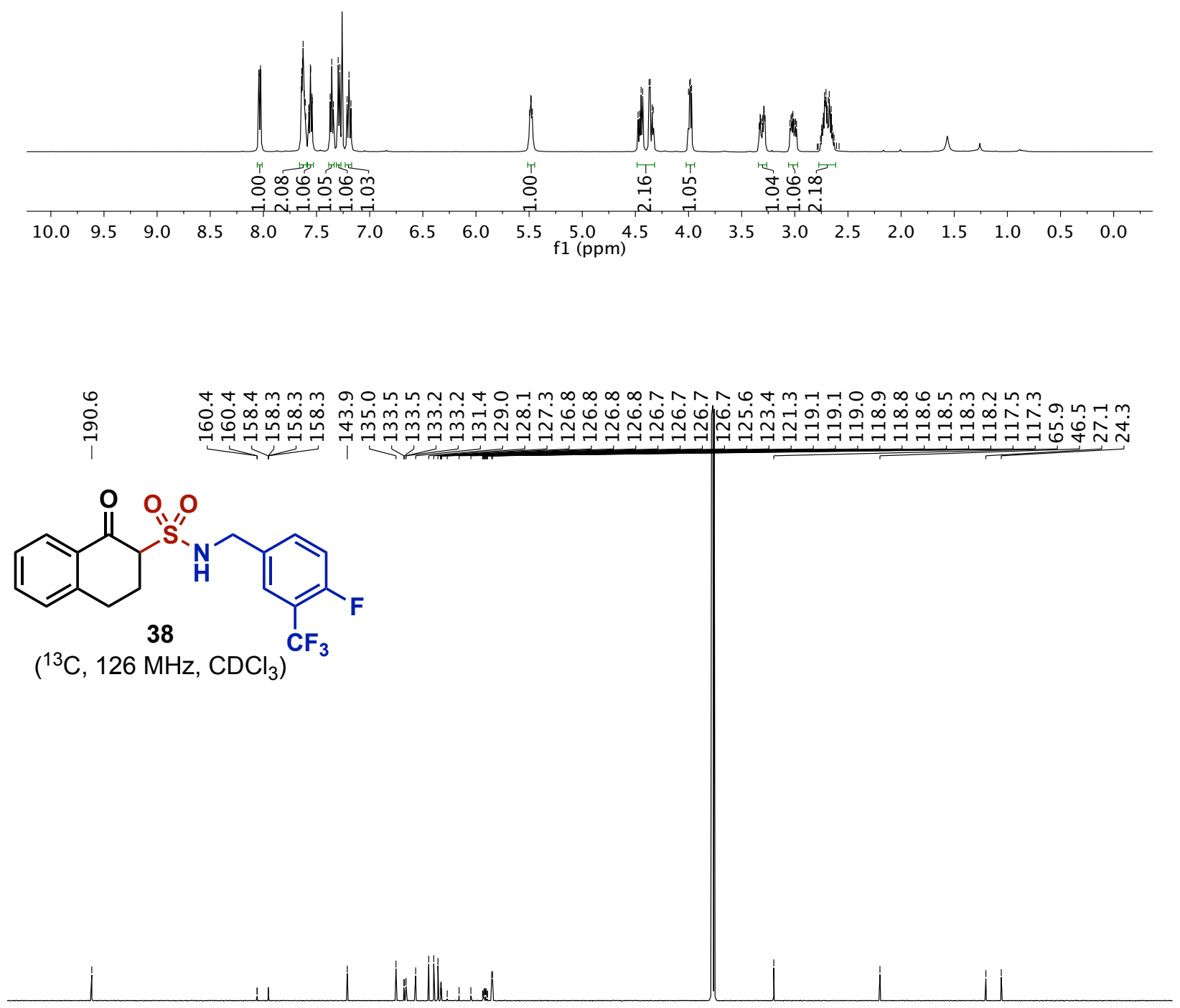

$\begin{array}{lllllllllllllllllllll}200 & 190 & 180 & 170 & 160 & 150 & 140 & 130 & 120 & 110 \underset{\mathrm{f} 1(\mathrm{ppm})}{100} 90 & 80 & 70 & 60 & 50 & 40 & 30 & 20 & 10 & 0\end{array}$ 


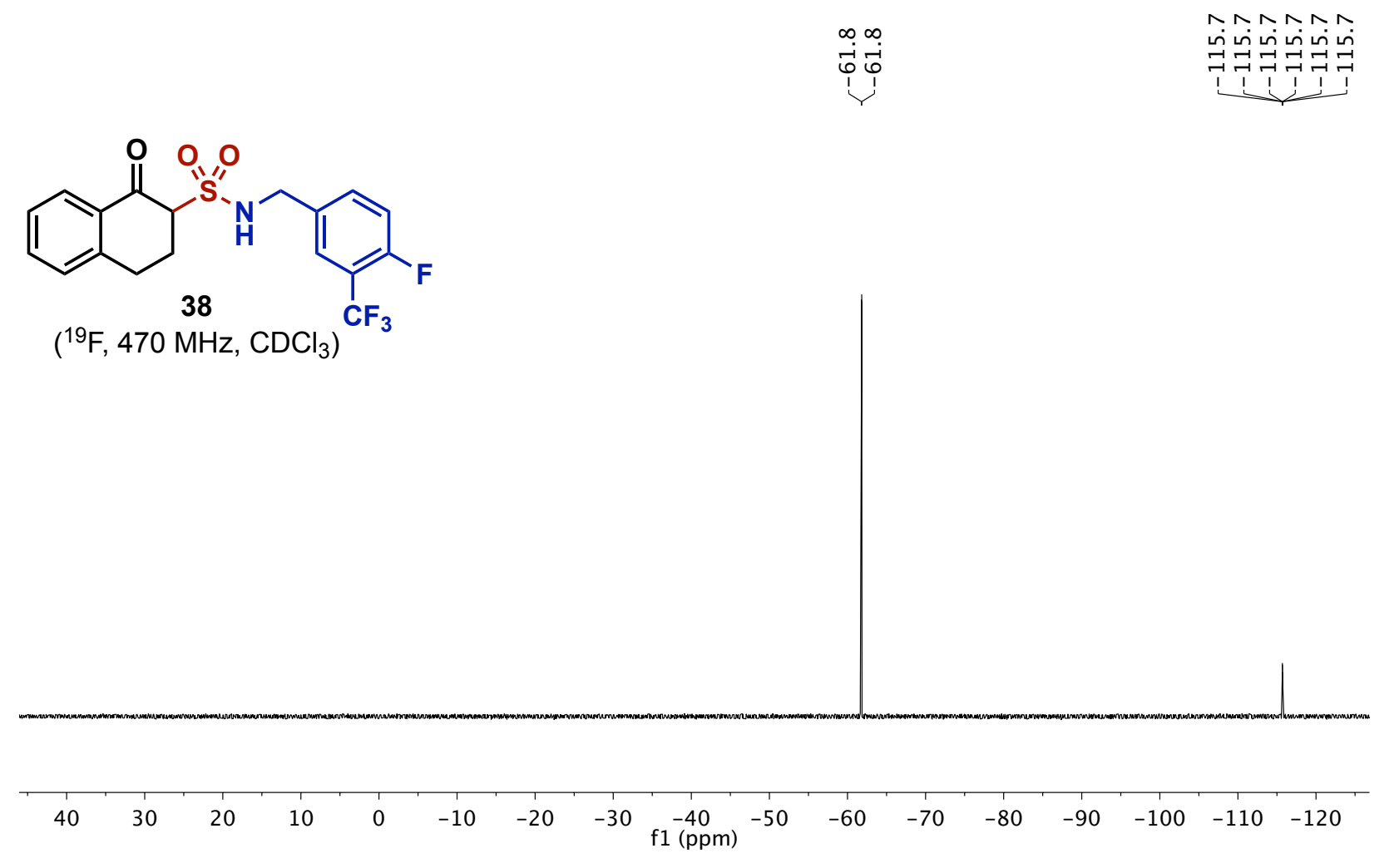




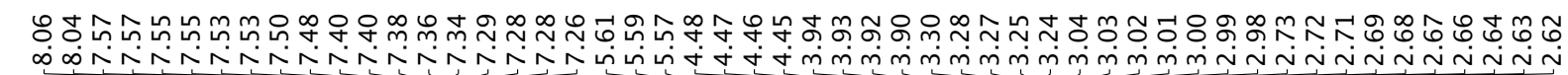<smiles>CC#CCCCC1CCc2ccccc2C1=O</smiles>

39

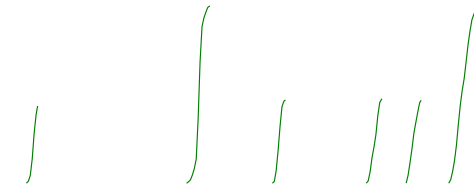

$\left({ }^{1} \mathrm{H}, 400 \mathrm{MHz}, \mathrm{CDCl}_{3}\right)$
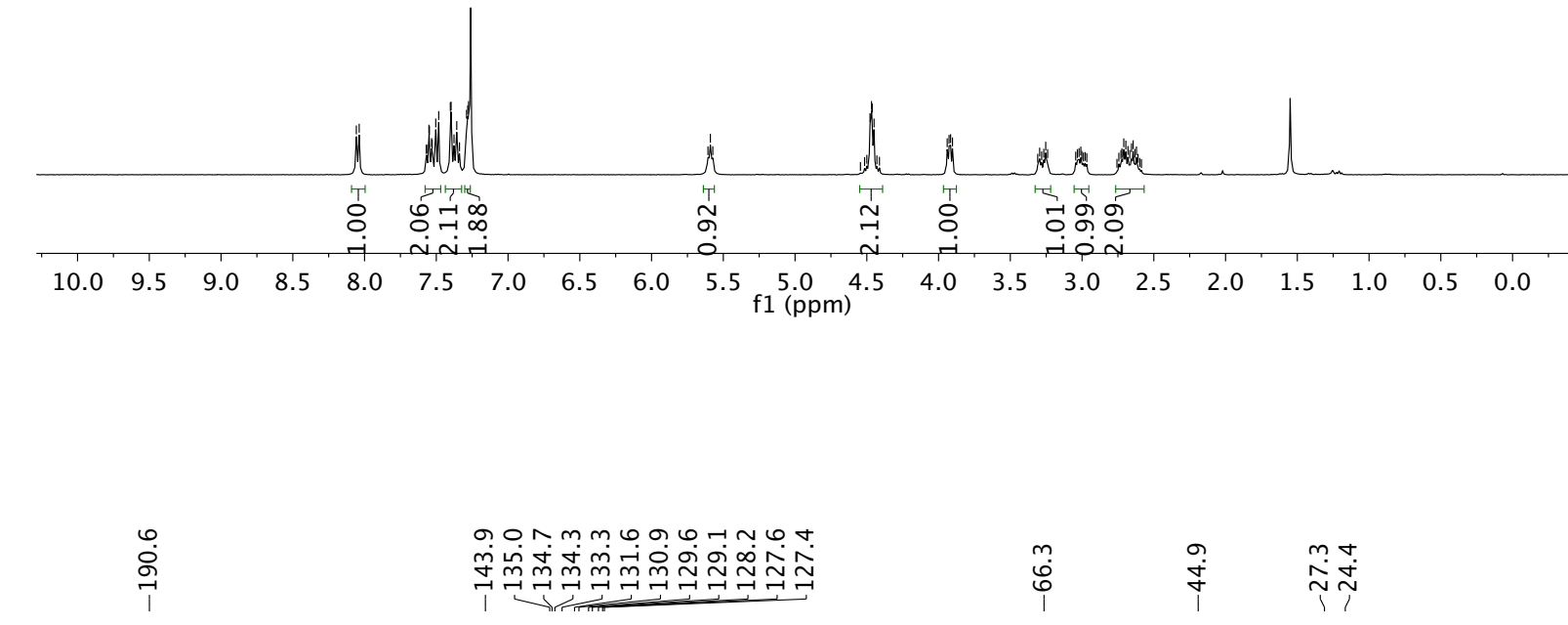

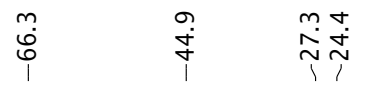<smiles>O=C1c2ccccc2CCC1S(=O)(=O)NCc1ccc(Cl)cc1Cl</smiles>

39

$\left({ }^{13} \mathrm{C}, 126 \mathrm{MHz}, \mathrm{CDCl}_{3}\right)$

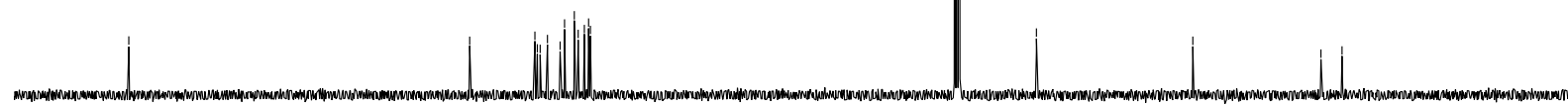

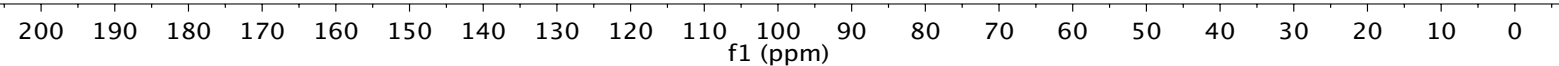




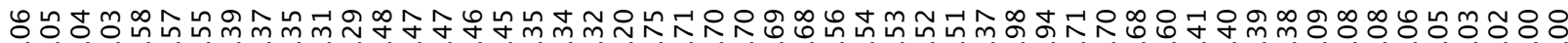

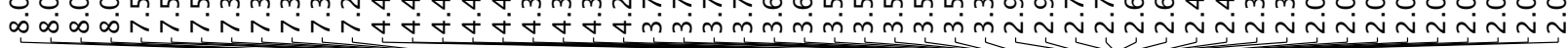<smiles>C=CC[C@H]1CCCN1S(=O)(=O)C1CCc2ccccc2C1=O</smiles>

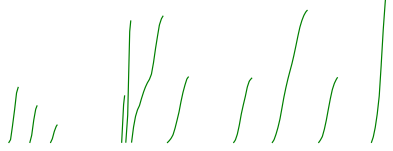

$\left({ }^{1} \mathrm{H}, 400 \mathrm{MHz}, \mathrm{C}_{2} \mathrm{D}_{2} \mathrm{Cl}_{4}\right)$

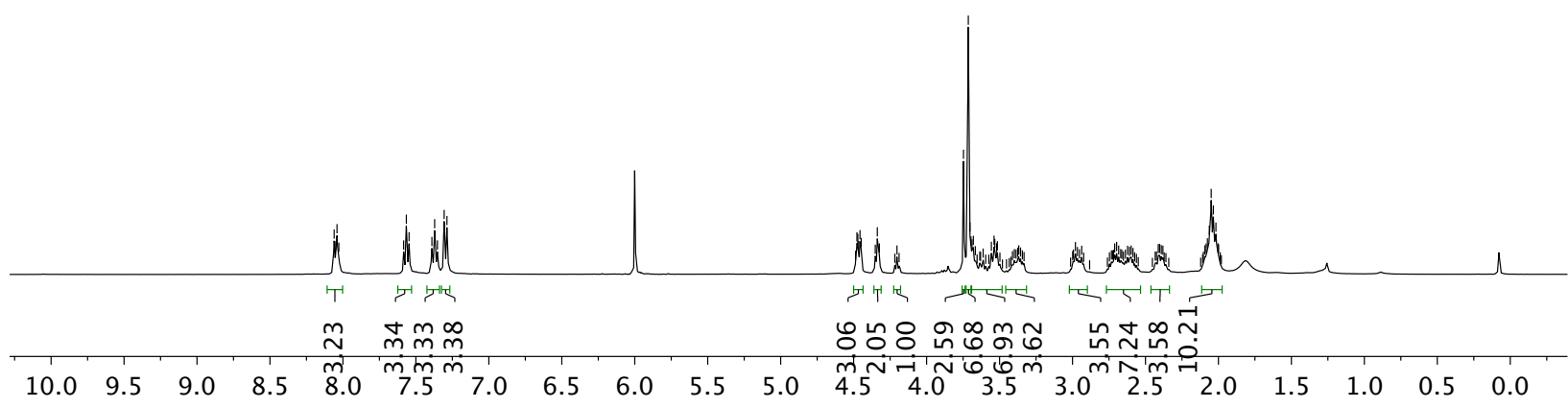

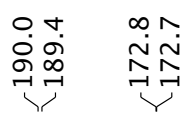

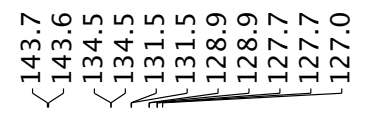

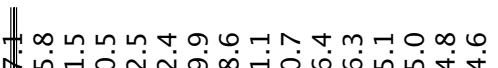
|<smiles>COC(=O)[C@H]1CCCN1S(=O)(=O)C1CCc2ccccc2C1=O</smiles>

40

$\left({ }^{13} \mathrm{C}, 126 \mathrm{MHz}, \mathrm{C}_{2} \mathrm{D}_{2} \mathrm{Cl}_{4}\right)$

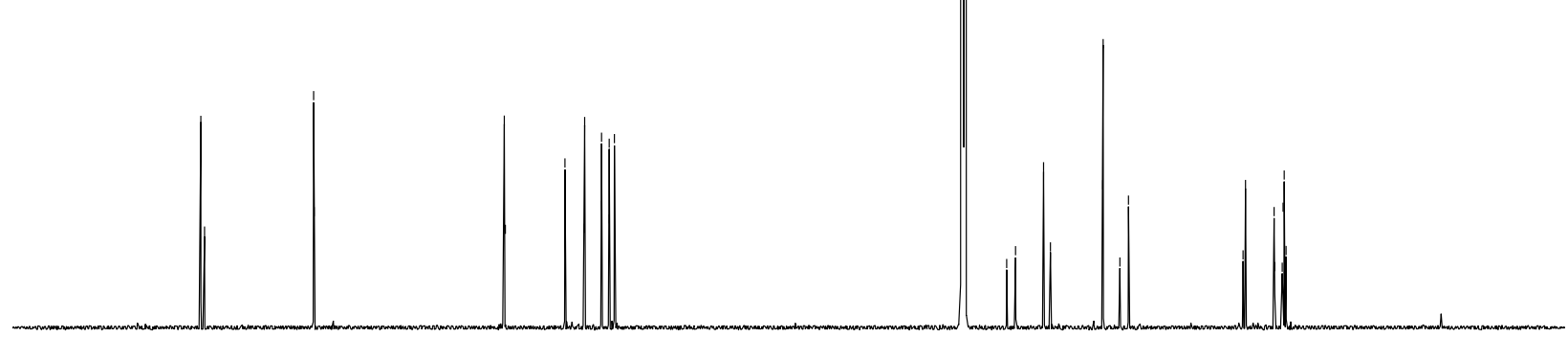

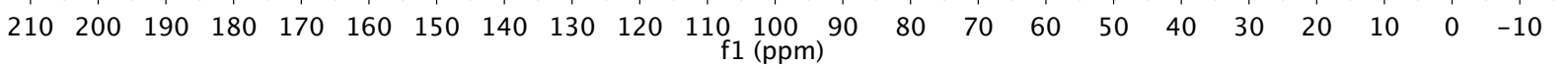




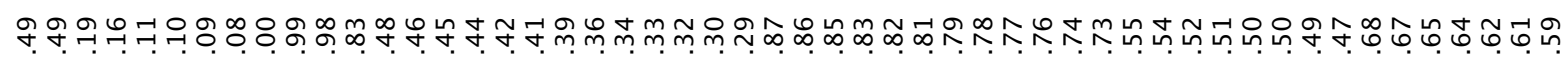

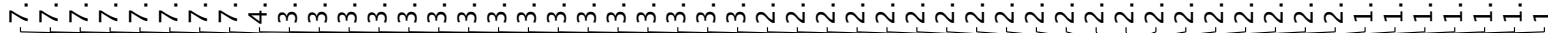<smiles>C=CC1CCCN(S(=O)(=O)C2CCc3ccc(OC)cc3C2=O)C1</smiles>

41

$\left({ }^{1} \mathrm{H}, 400 \mathrm{MHz}, \mathrm{CDCl}_{3}\right)$
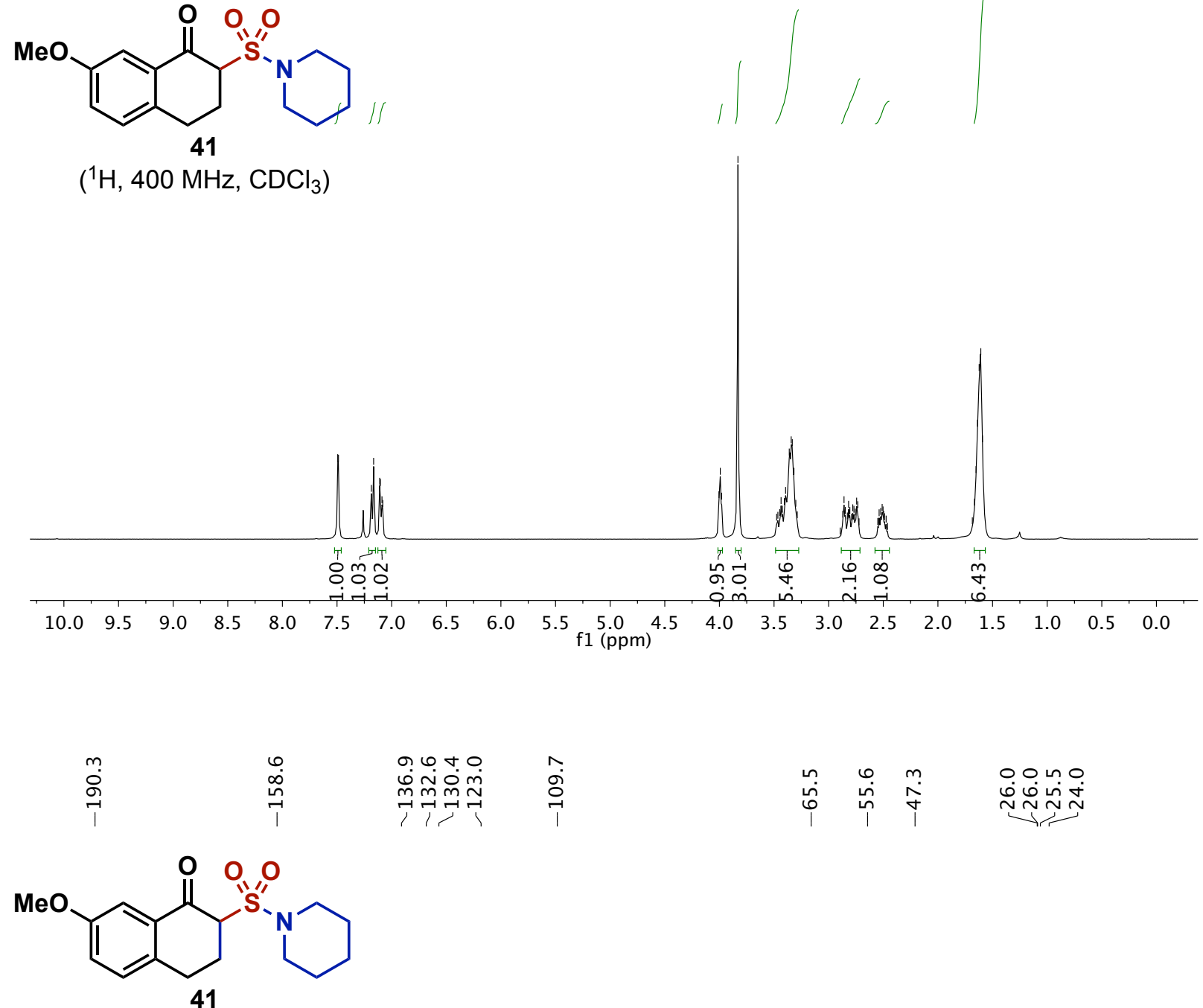

$\left({ }^{13} \mathrm{C}, 126 \mathrm{MHz}, \mathrm{CDCl}_{3}\right)$
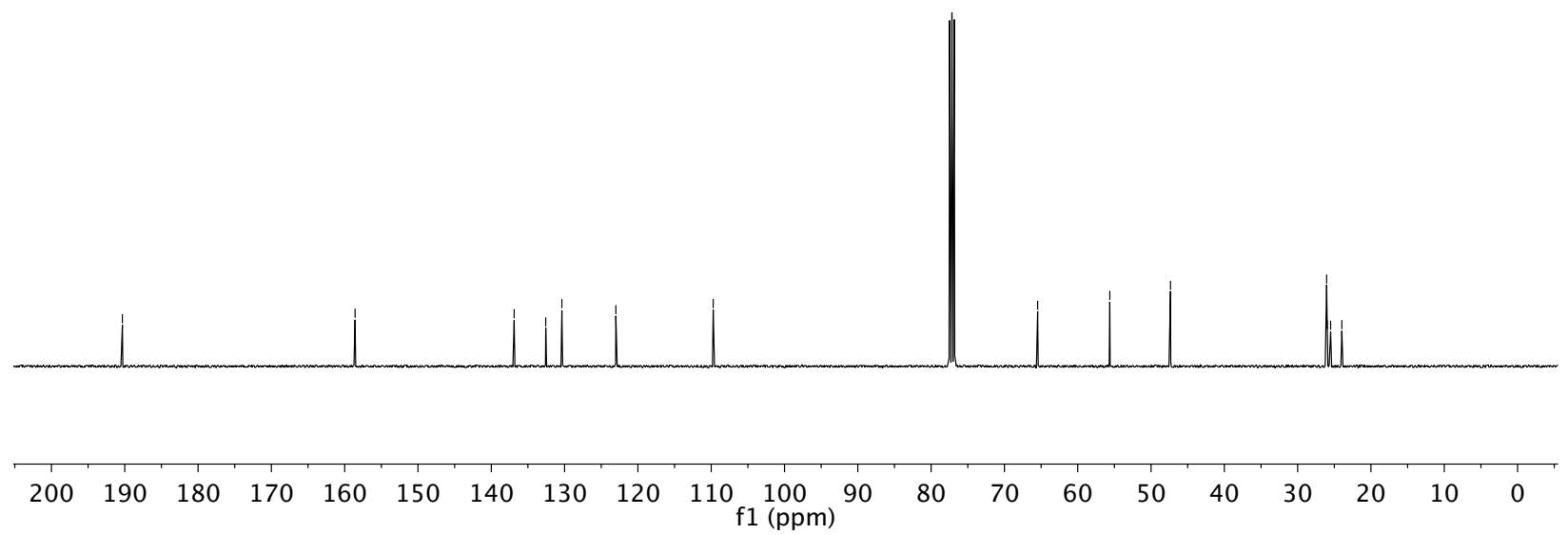


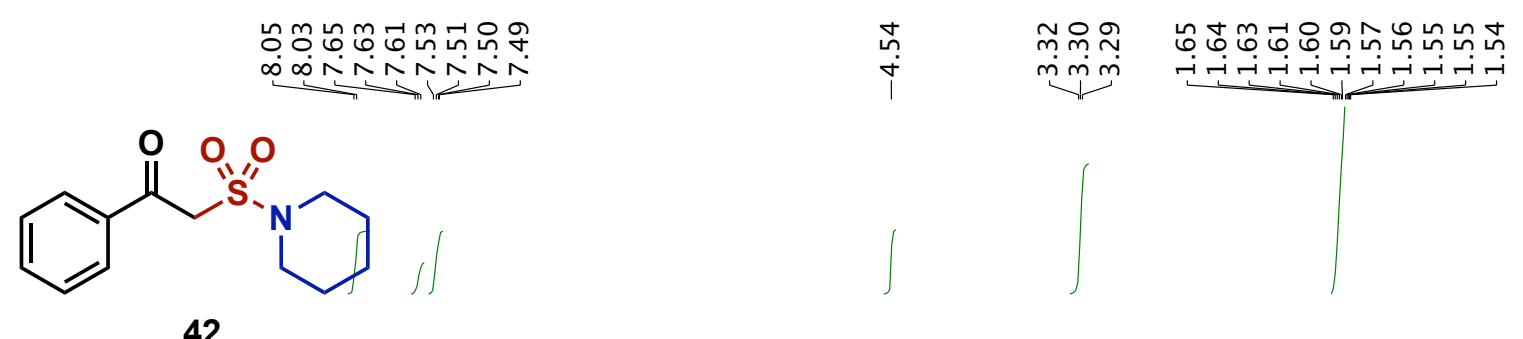

$\left({ }^{1} \mathrm{H}, 400 \mathrm{MHz}, \mathrm{CDCl}_{3}\right)$
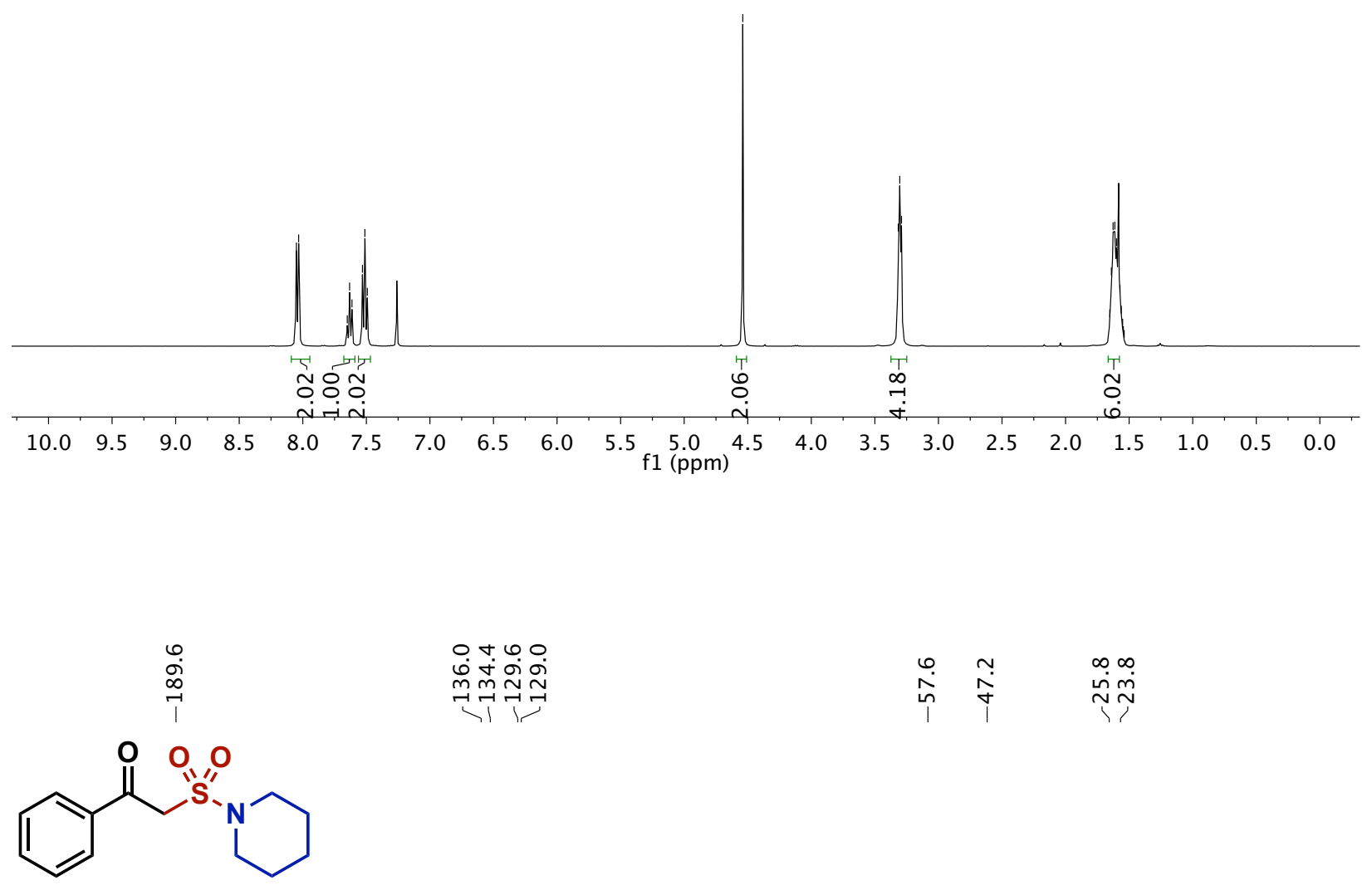

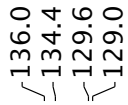

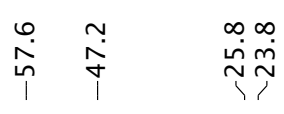

42

$\left({ }^{13} \mathrm{C}, 126 \mathrm{MHz}, \mathrm{CDCl}_{3}\right)$

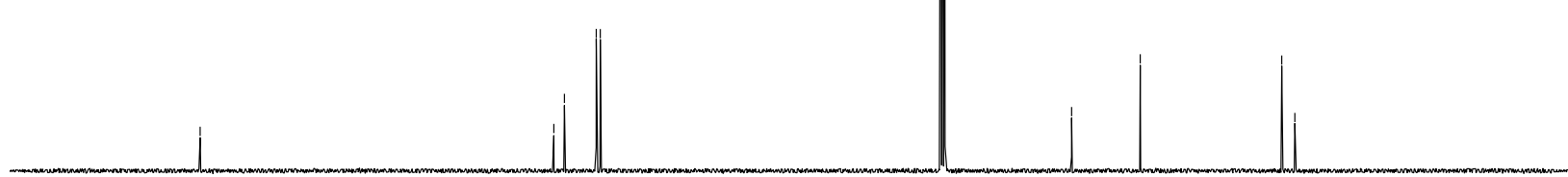

$\begin{array}{lllllllllllllllllllllllllllllll}210 & 200 & 190 & 180 & 170 & 160 & 150 & 140 & 130 & 120 & 110 & 100 & 90 & 80 & 70 & 60 & 50 & 40 & 30 & 20 & 10 & 0 & -10\end{array}$ 
<smiles>O=C(CS(=O)(=O)N1CCCCC1)c1cc(F)cc(F)c1</smiles>

$\left({ }^{1} \mathrm{H}, 400 \mathrm{MHz}, \mathrm{CDCl}_{3}\right)$
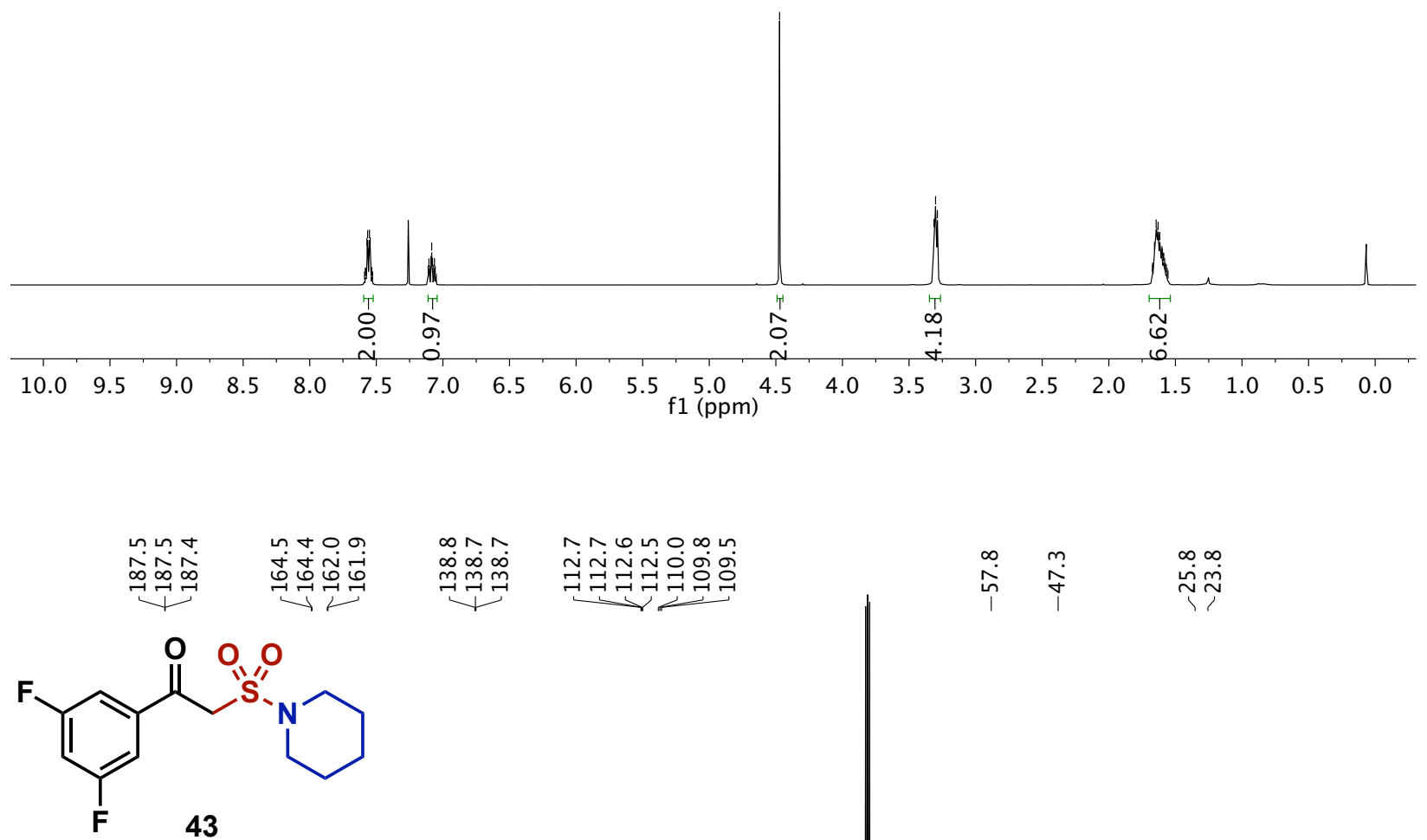

$\left({ }^{13} \mathrm{C}, 126 \mathrm{MHz}, \mathrm{CDCl}_{3}\right)$

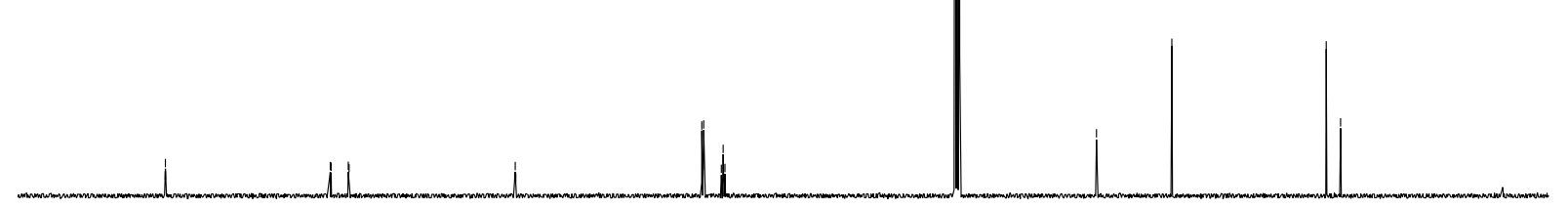

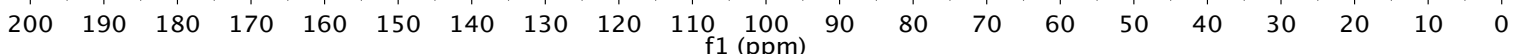




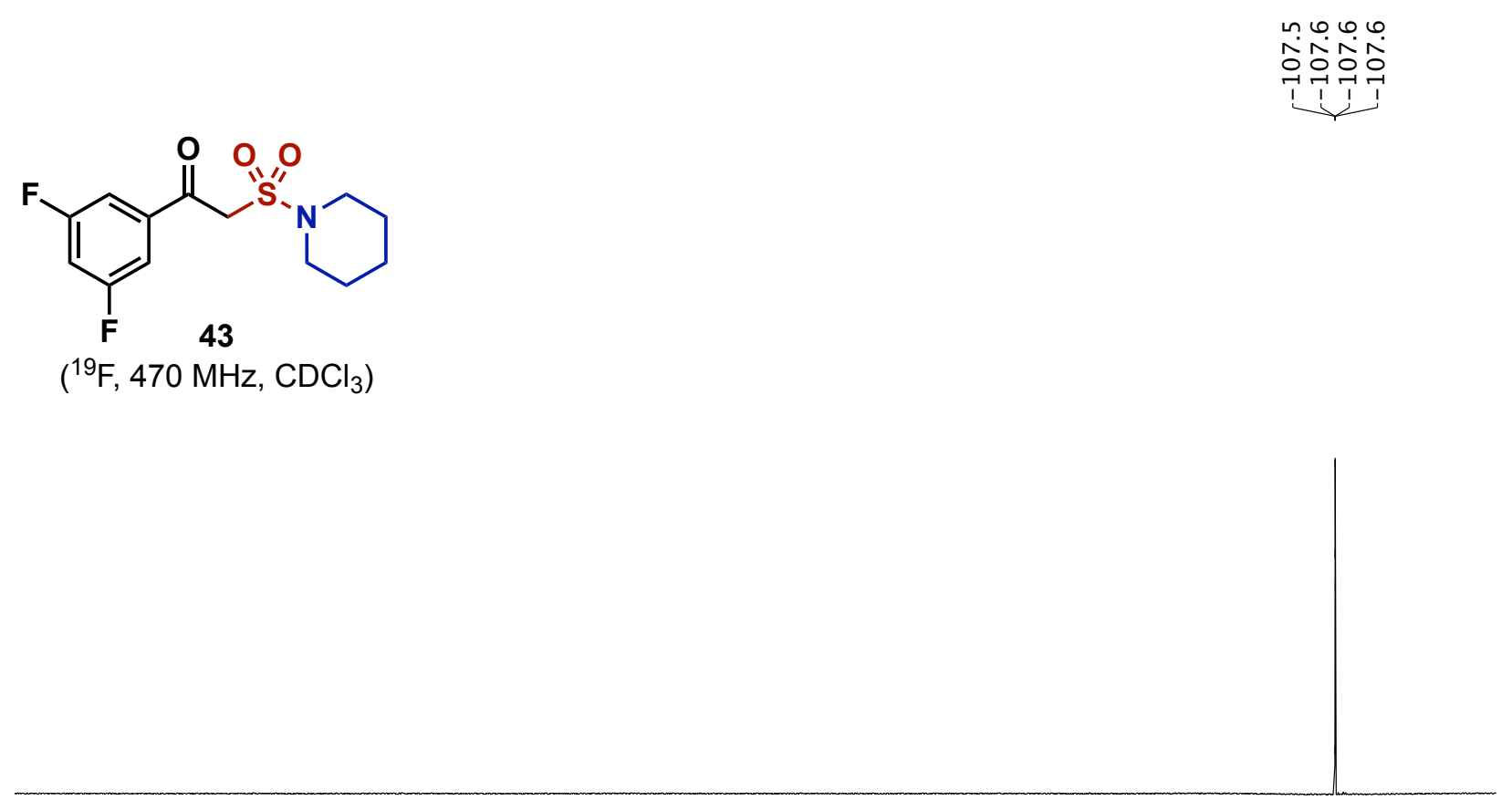

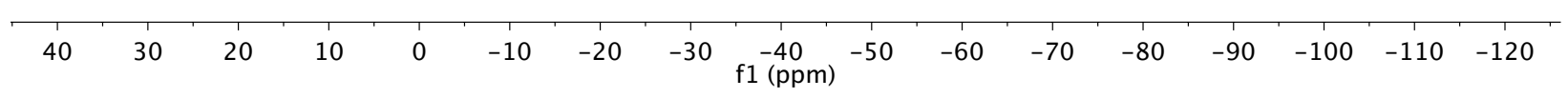



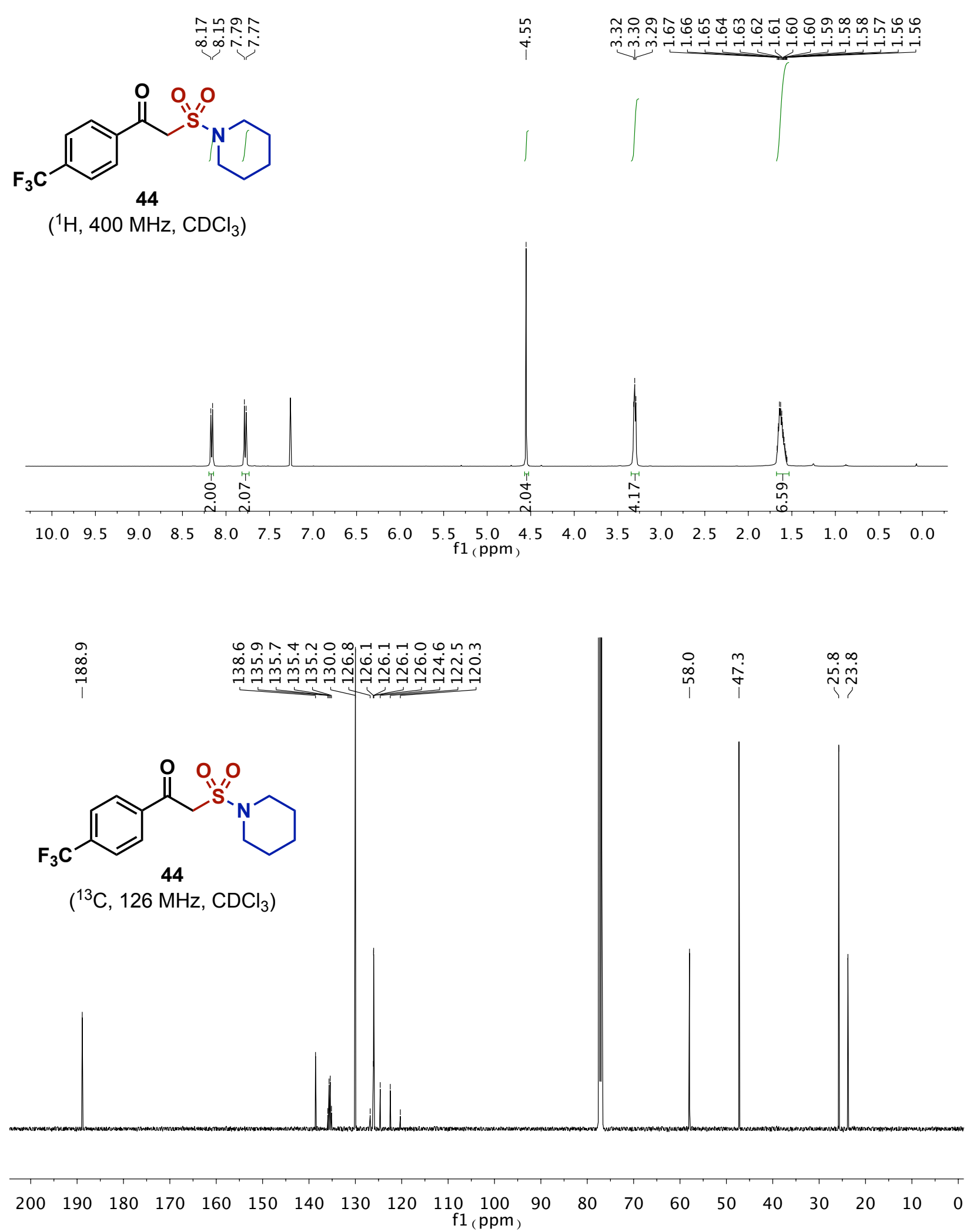

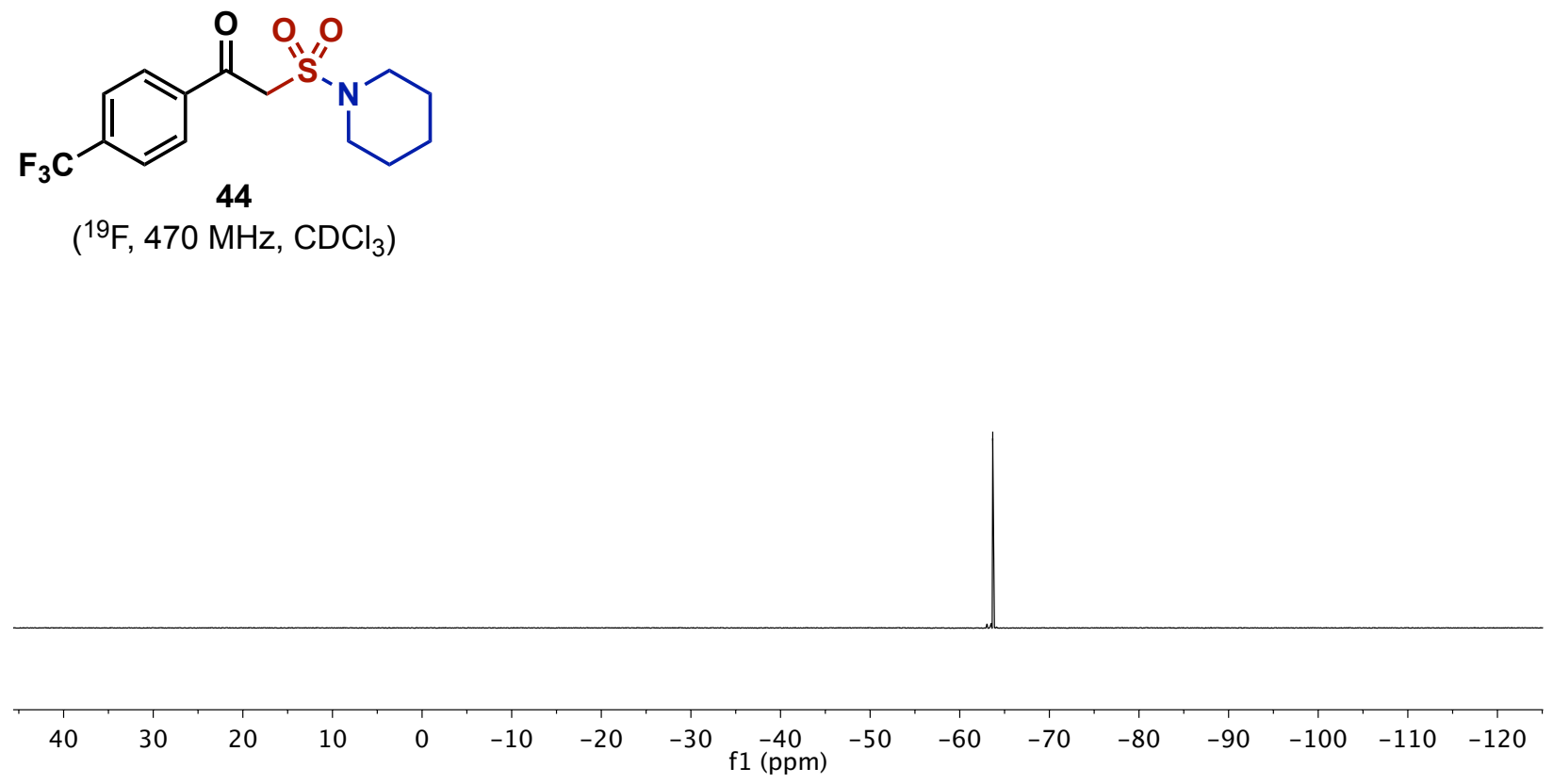

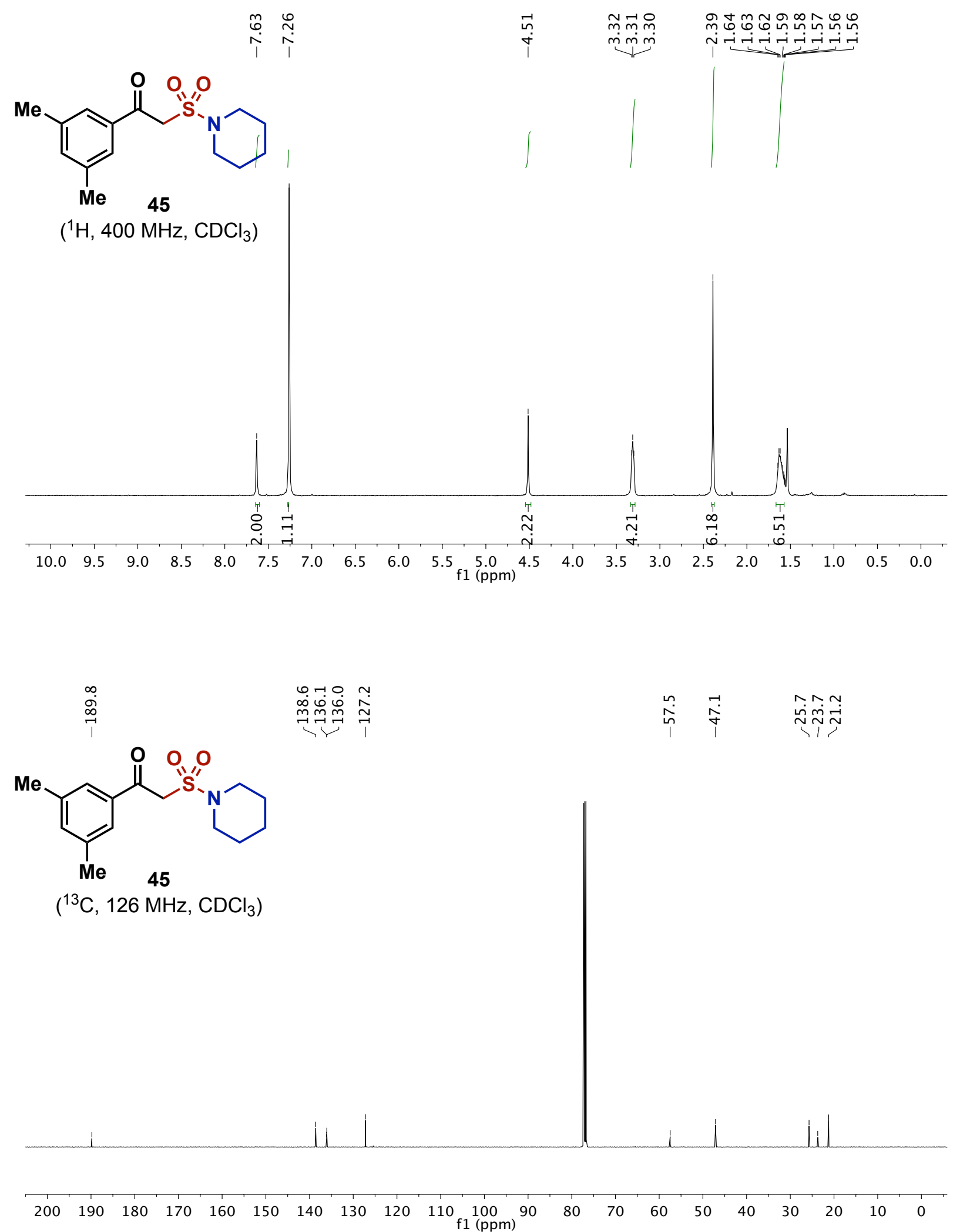


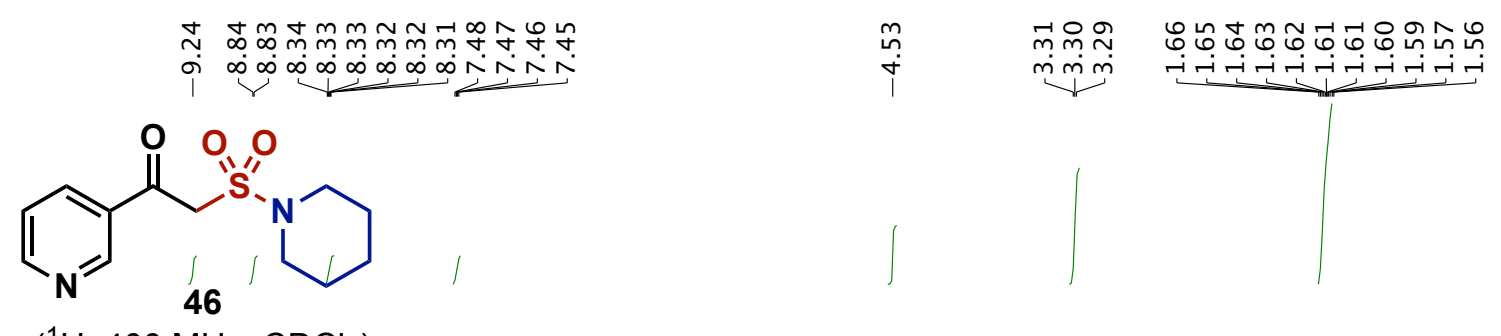

$\left({ }^{1} \mathrm{H}, 400 \mathrm{MHz}, \mathrm{CDCl}_{3}\right)$
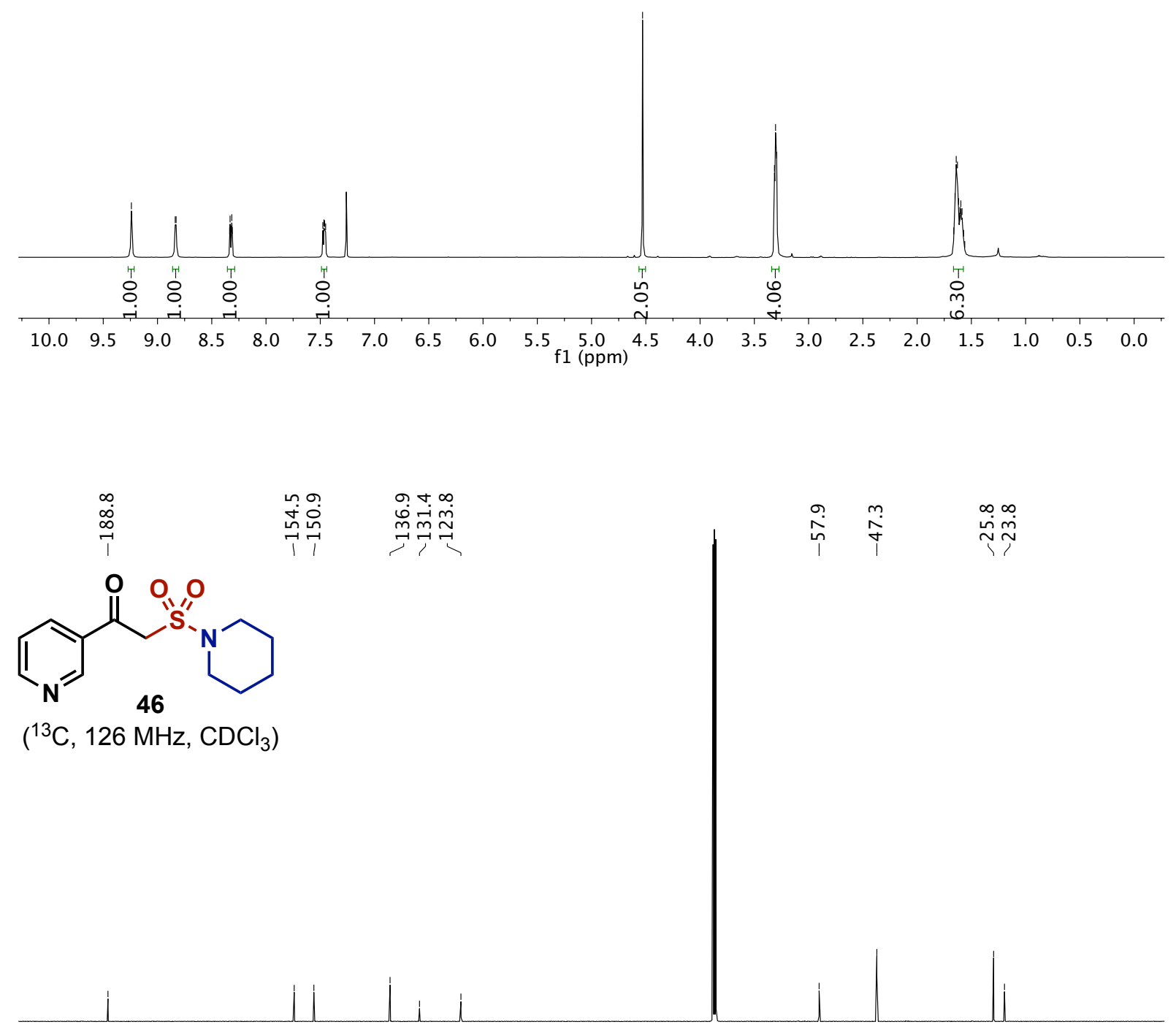

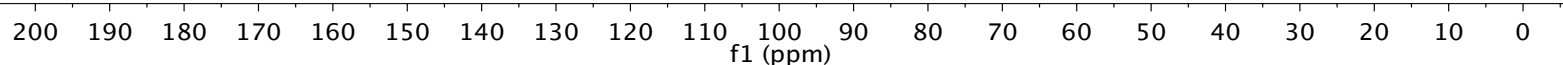




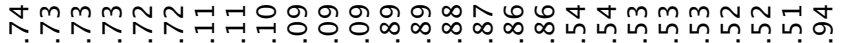
$\infty \infty \infty \infty \infty \infty \infty \infty \infty \infty \infty \infty \infty+\infty)+N$<smiles>CS(=O)(=O)N1CCCCC1</smiles>

47

$\left({ }^{1} \mathrm{H}, 400 \mathrm{MHz}, \mathrm{CDCl}_{3}\right)$

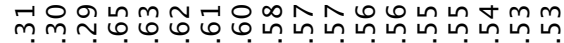
mmm

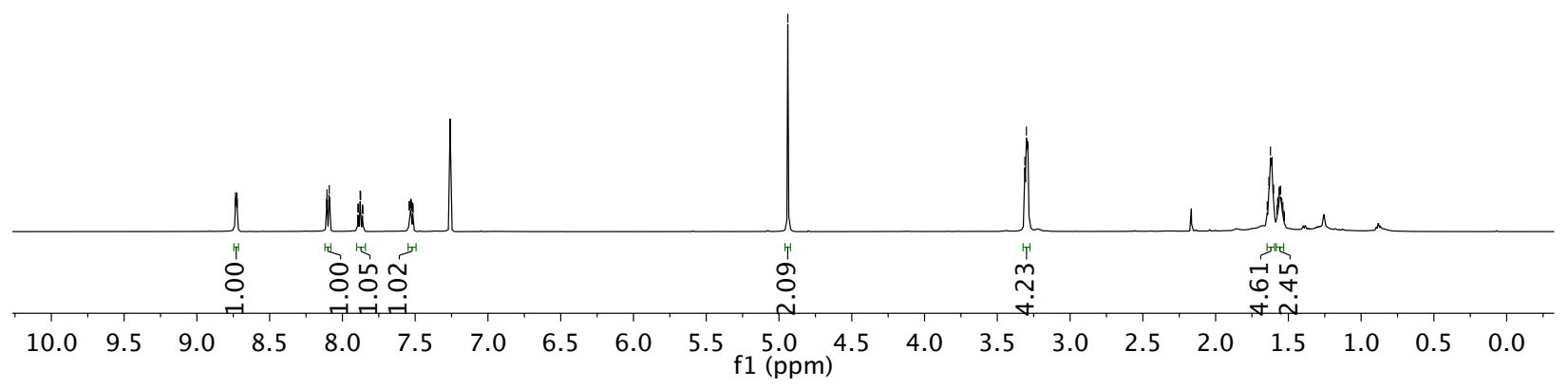

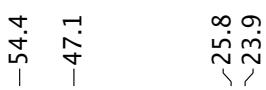<smiles>O=C(CS(=O)(=O)N1CCCCC1)c1ccccn1</smiles>

$\left({ }^{13} \mathrm{C}, 126 \mathrm{MHz}, \mathrm{CDCl}_{3}\right)$
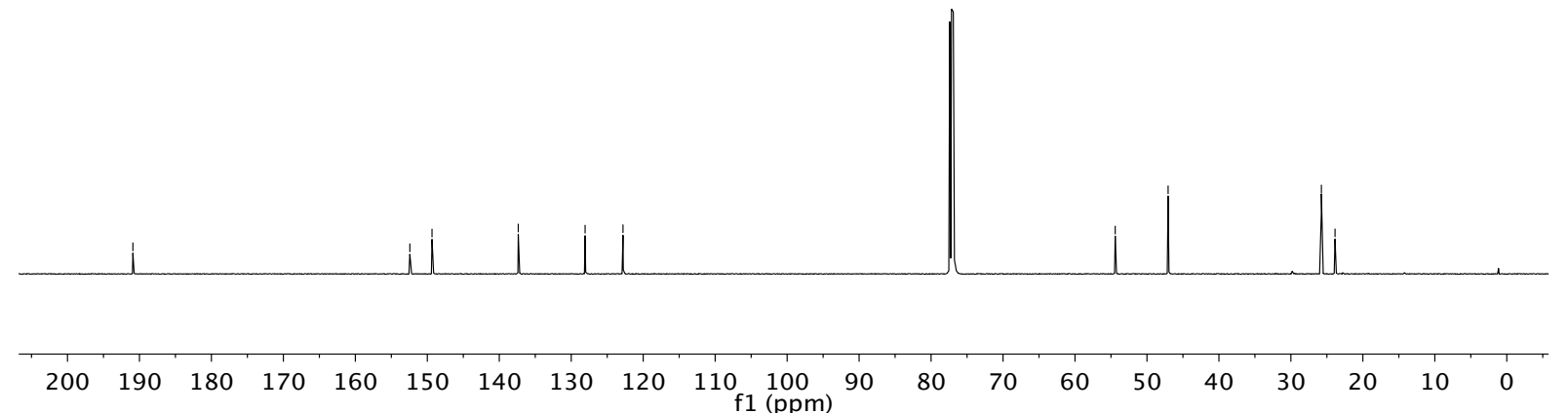


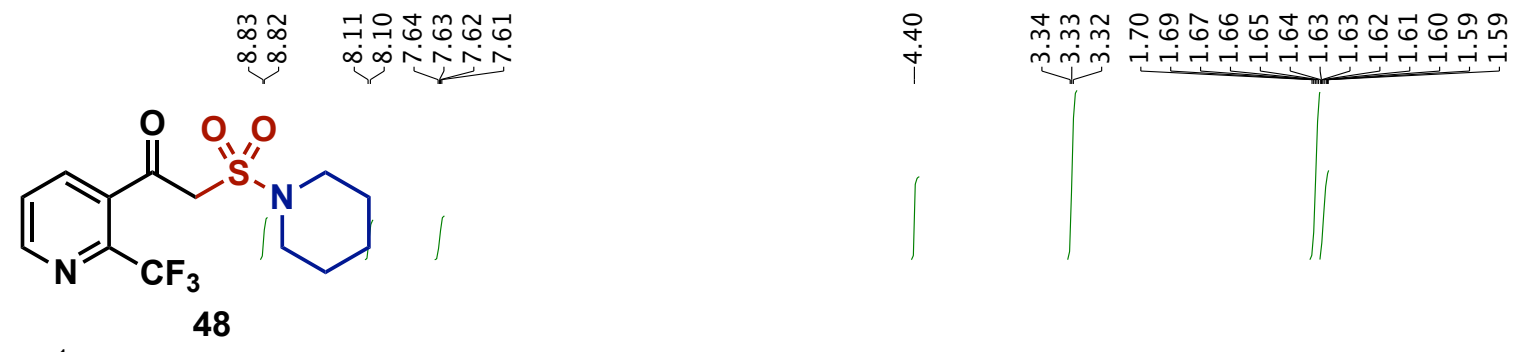

$\left({ }^{1} \mathrm{H}, 400 \mathrm{MHz}, \mathrm{CDCl}_{3}\right)$
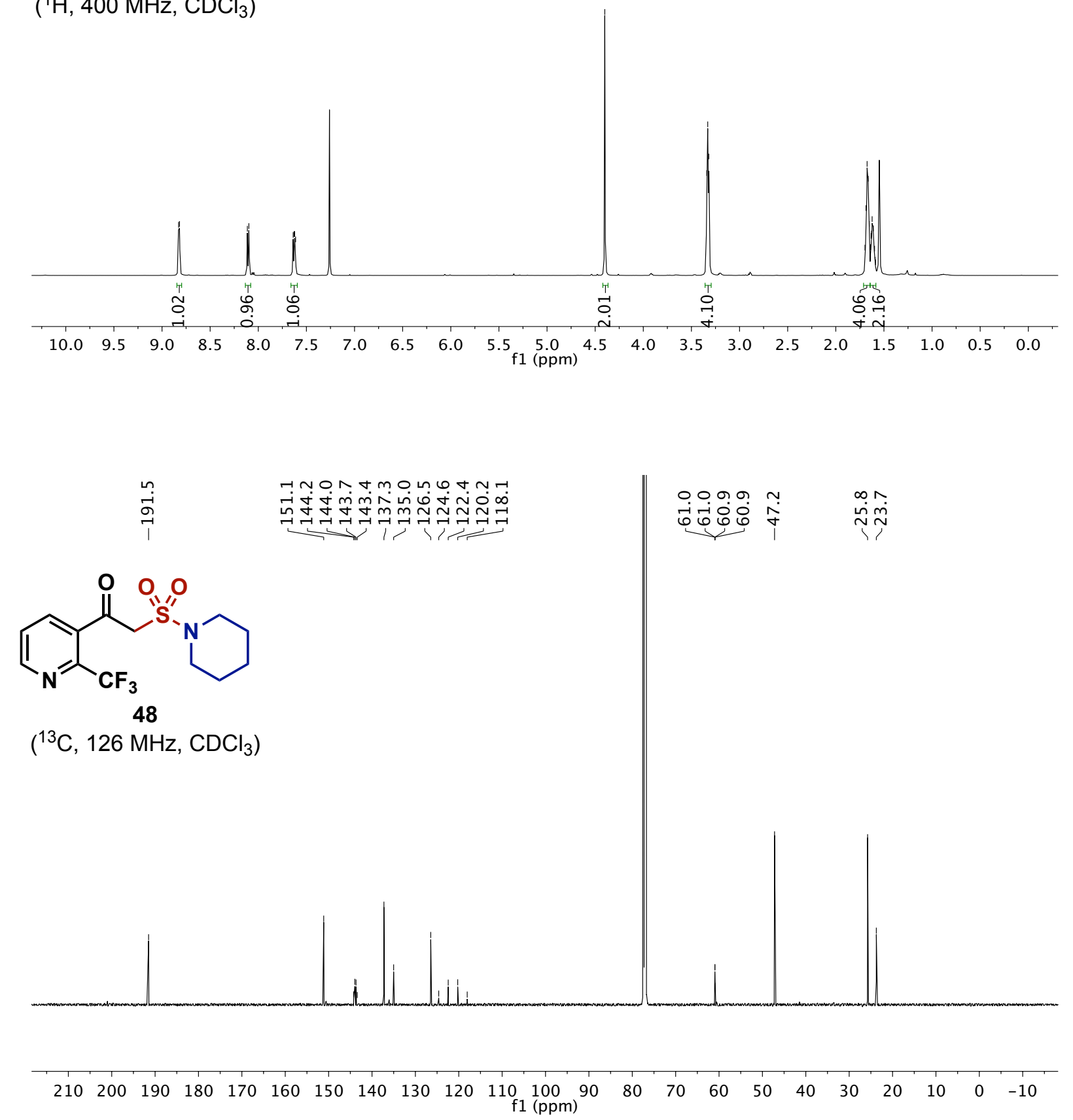


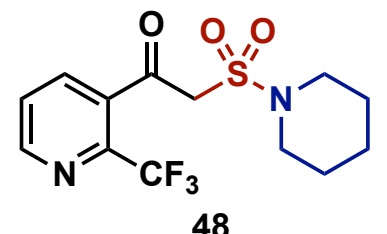

$\left({ }^{19} \mathrm{~F}, 470 \mathrm{MHz}, \mathrm{CDCl}_{3}\right)$

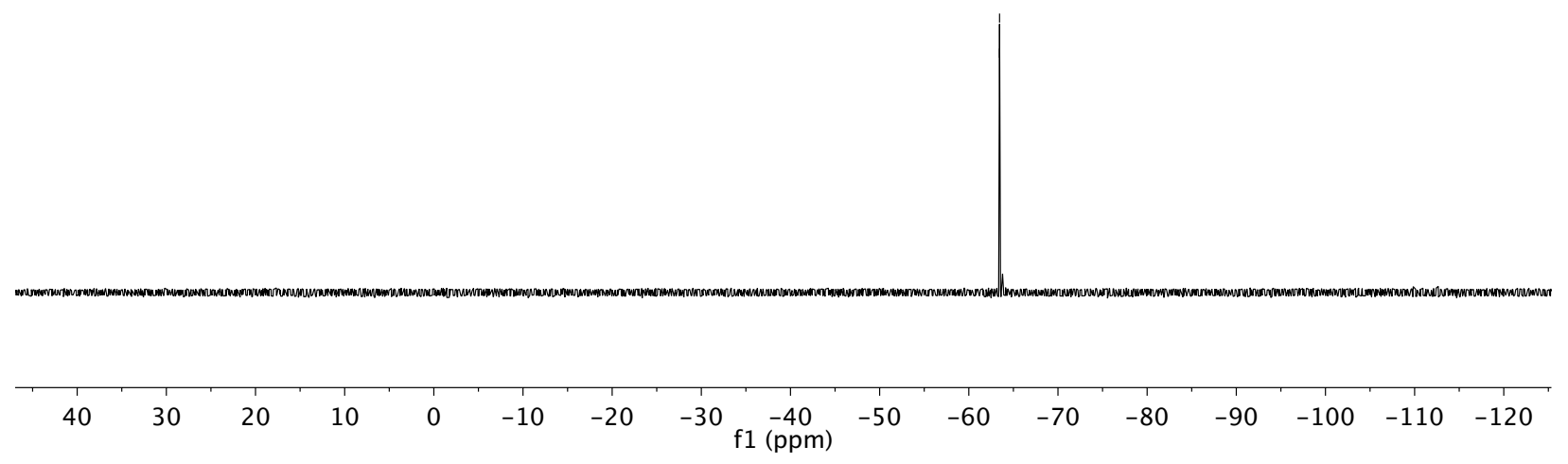




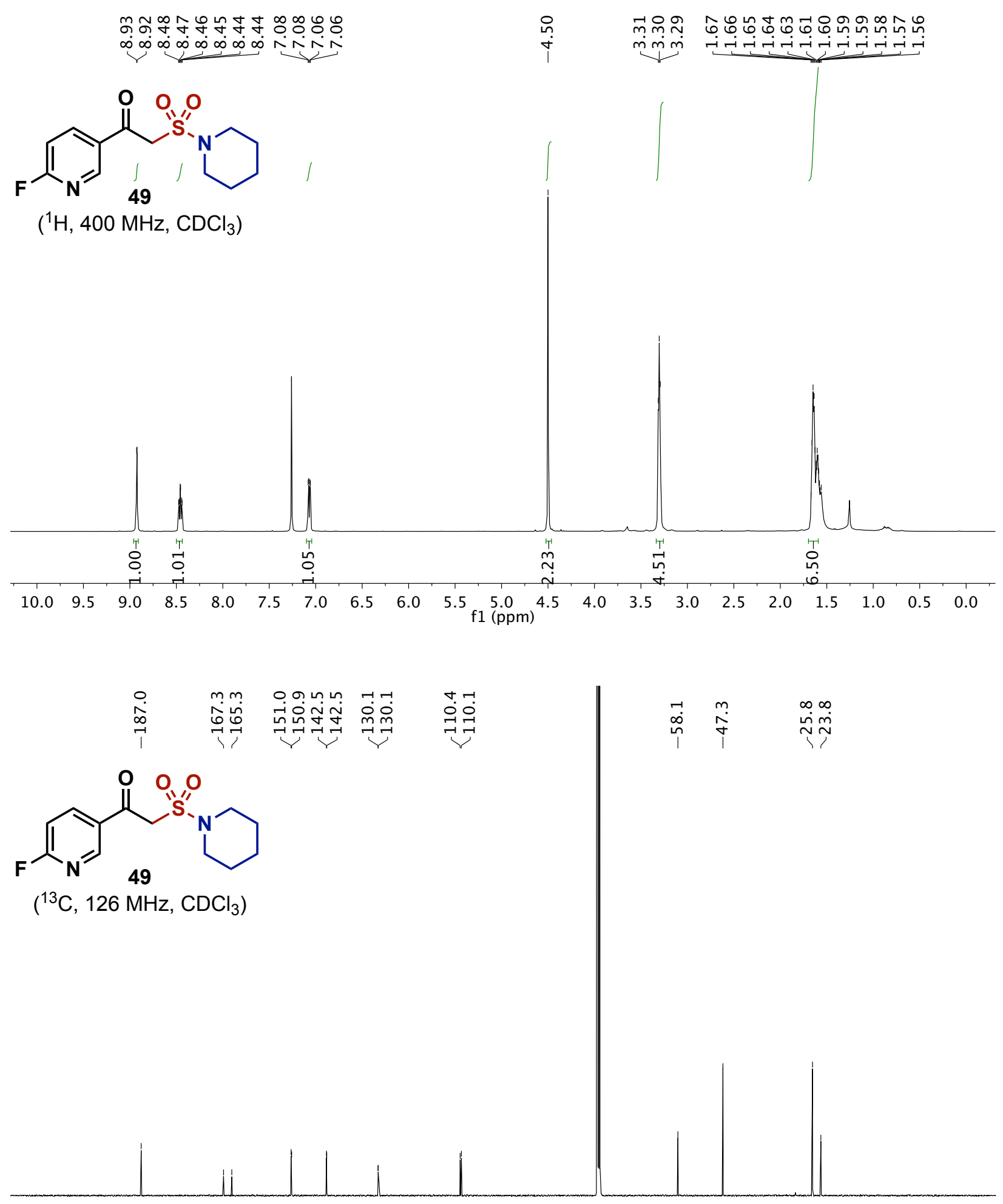

$\begin{array}{lllllllllllllllllllllll}210 & 200 & 190 & 180 & 170 & 160 & 150 & 140 & 130 & 120 & 110 \\ \mathrm{f} 1 \mathrm{fpm}) & 100 & 90 & 80 & 70 & 60 & 50 & 40 & 30 & 20 & 10 & 0 & -10\end{array}$ 

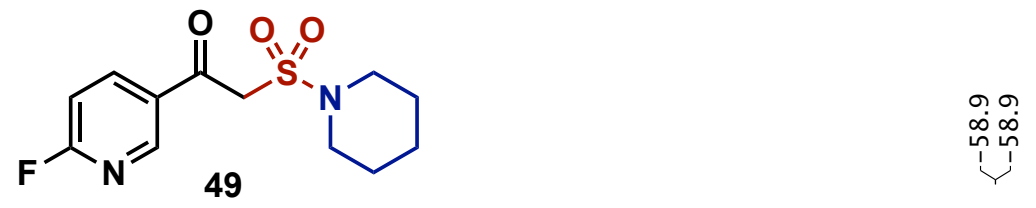

$\left({ }^{19} \mathrm{~F}, 470 \mathrm{MHz}, \mathrm{CDCl}_{3}\right)$

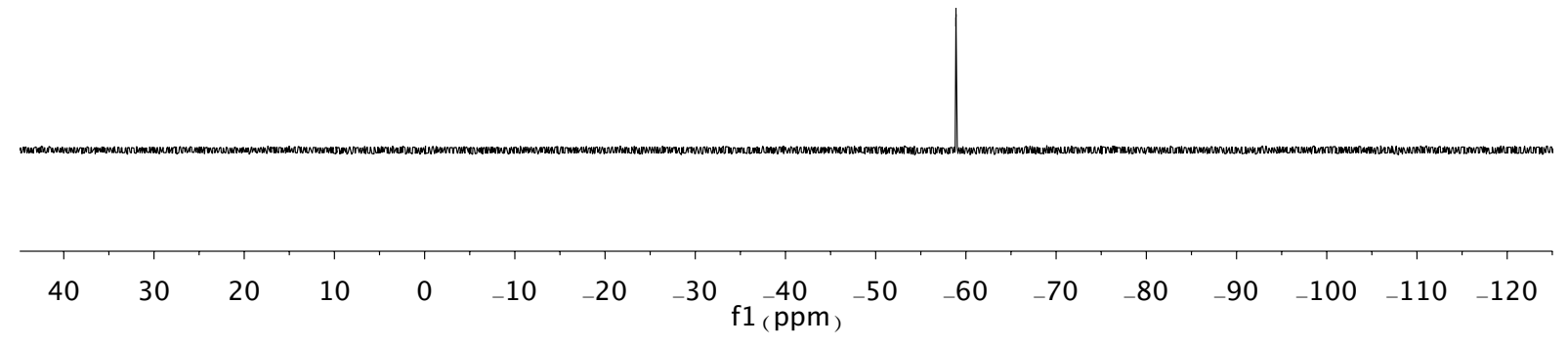



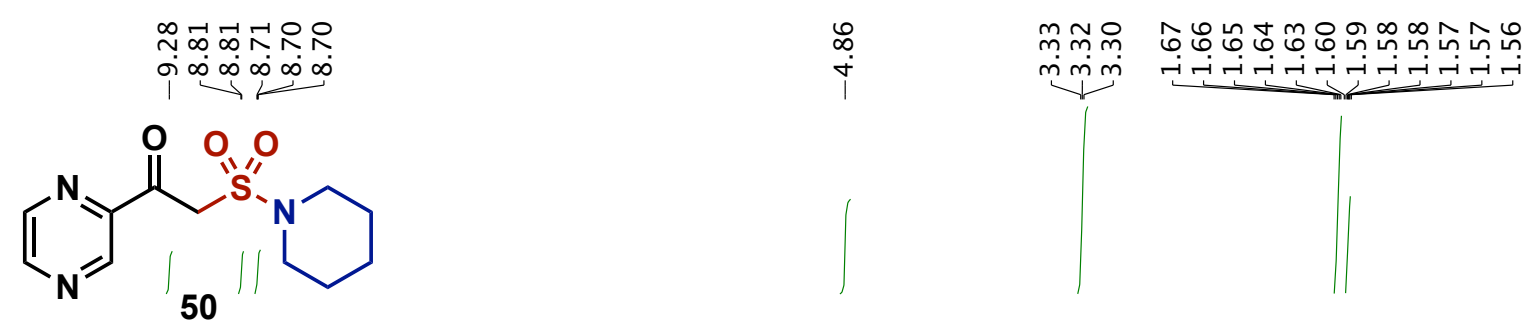

$\left({ }^{1} \mathrm{H}, 400 \mathrm{MHz}, \mathrm{CDCl}_{3}\right)$
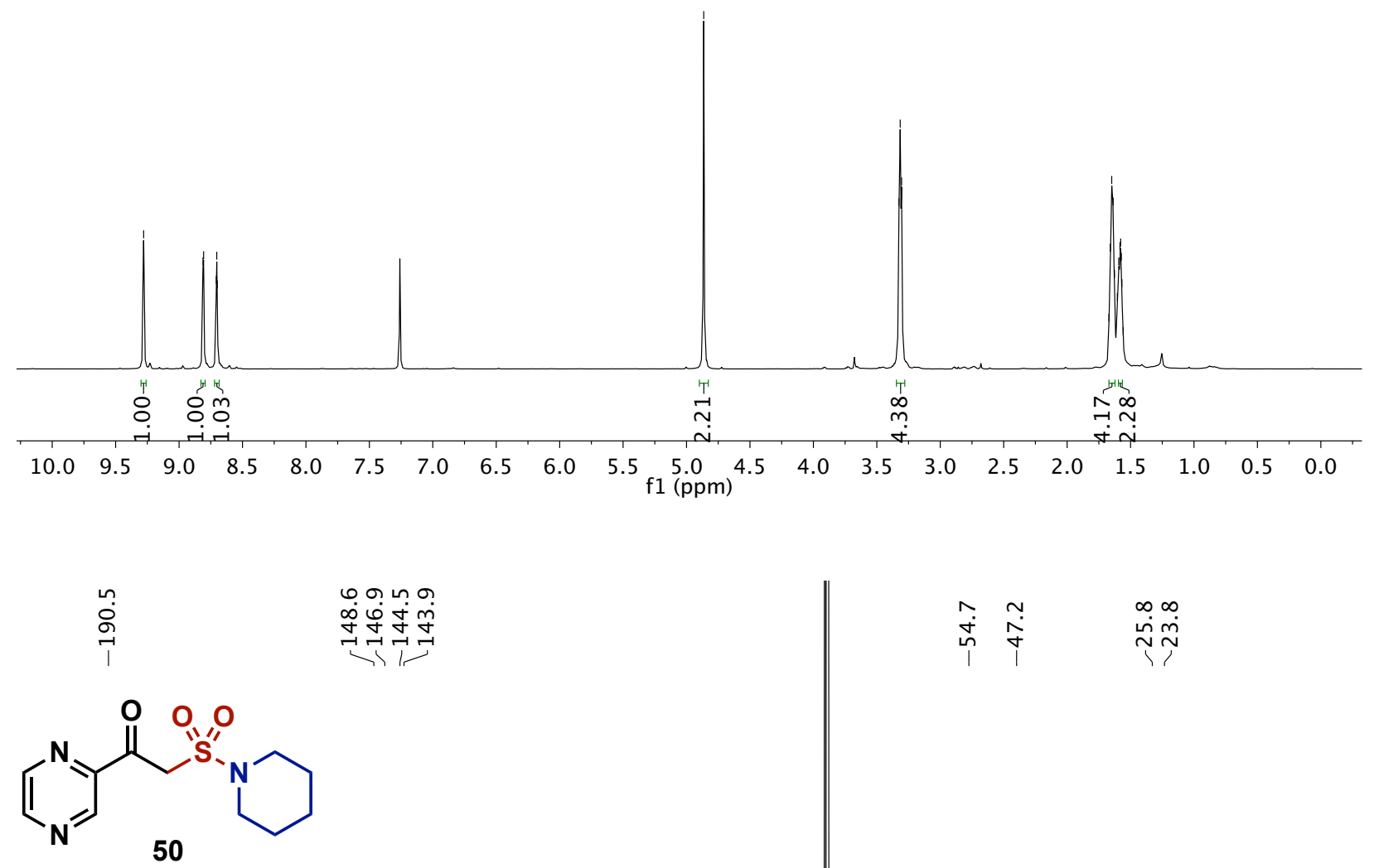

$\left({ }^{13} \mathrm{C}, 126 \mathrm{MHz}, \mathrm{CDCl}_{3}\right)$
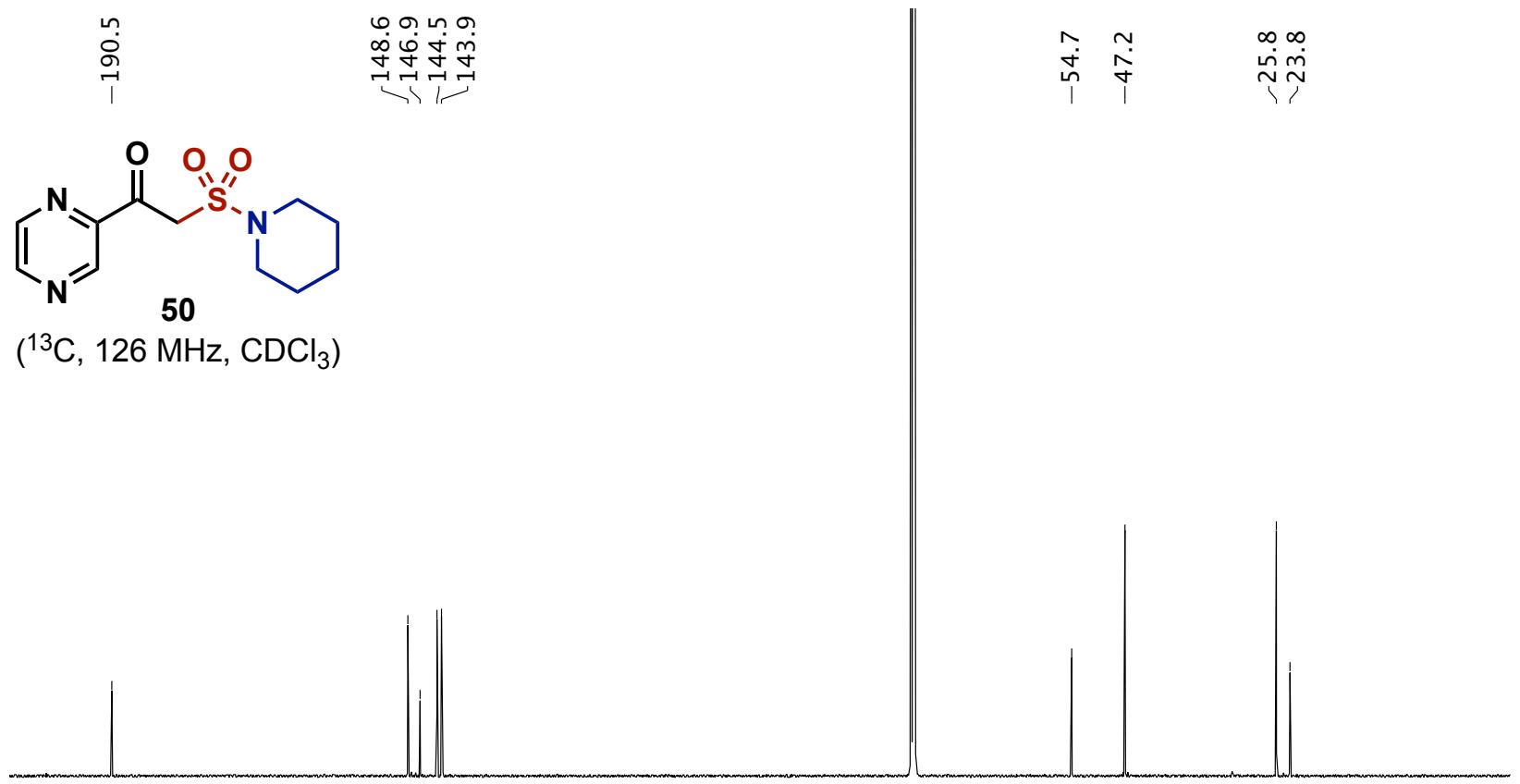

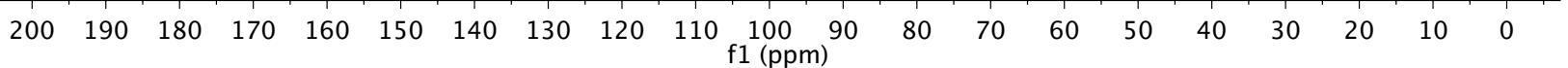




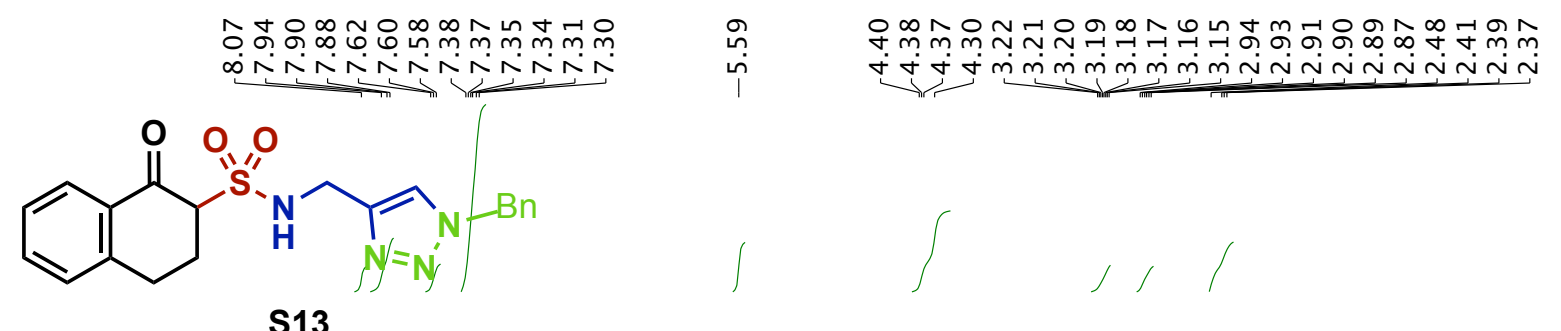

$\left({ }^{1} \mathrm{H}, 400 \mathrm{MHz}, d_{6}\right.$-DMSO)
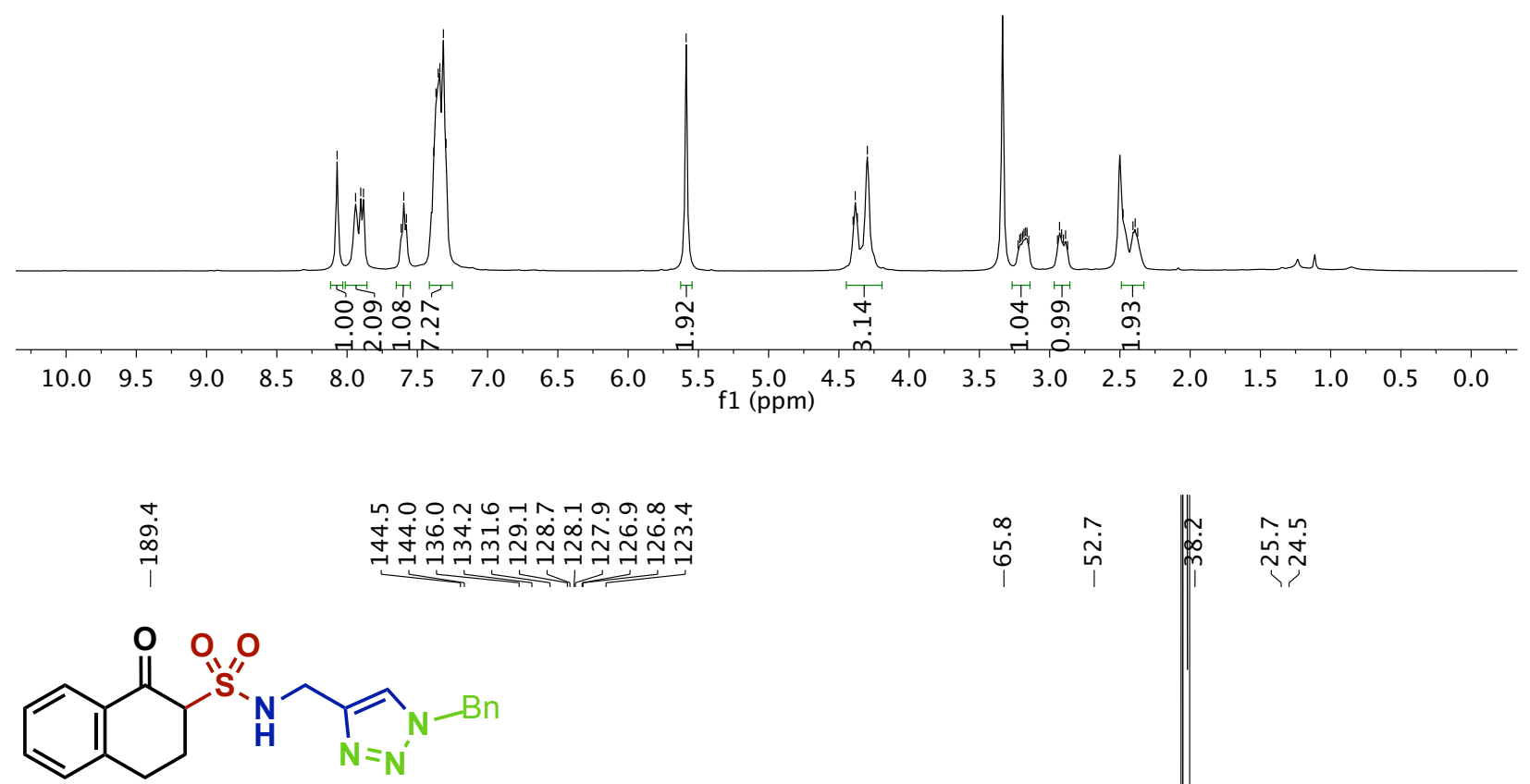

S13

$\left({ }^{13} \mathrm{C}, 126 \mathrm{MHz}, d_{6}\right.$-DMSO)

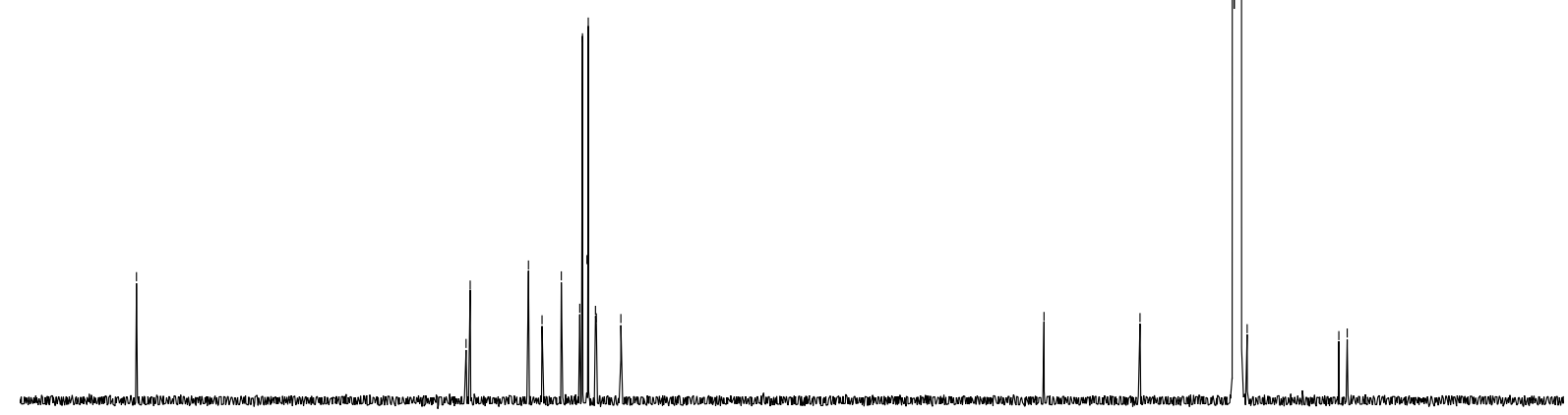

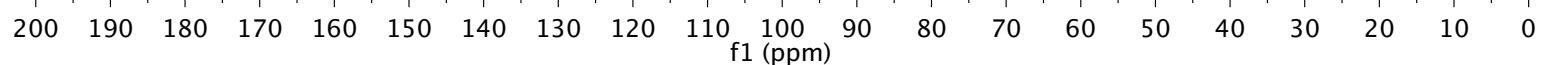




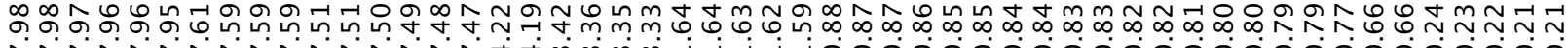

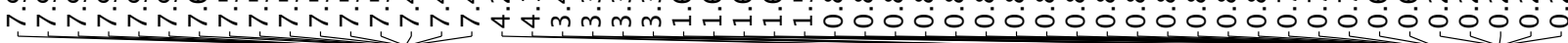<smiles>O=C(c1ccccc1)C(C1CC1)S(=O)(=O)N1CCCCC1</smiles>

54

$\left({ }^{1} \mathrm{H}, 400 \mathrm{MHz}, \mathrm{CDCl}_{3}\right)$

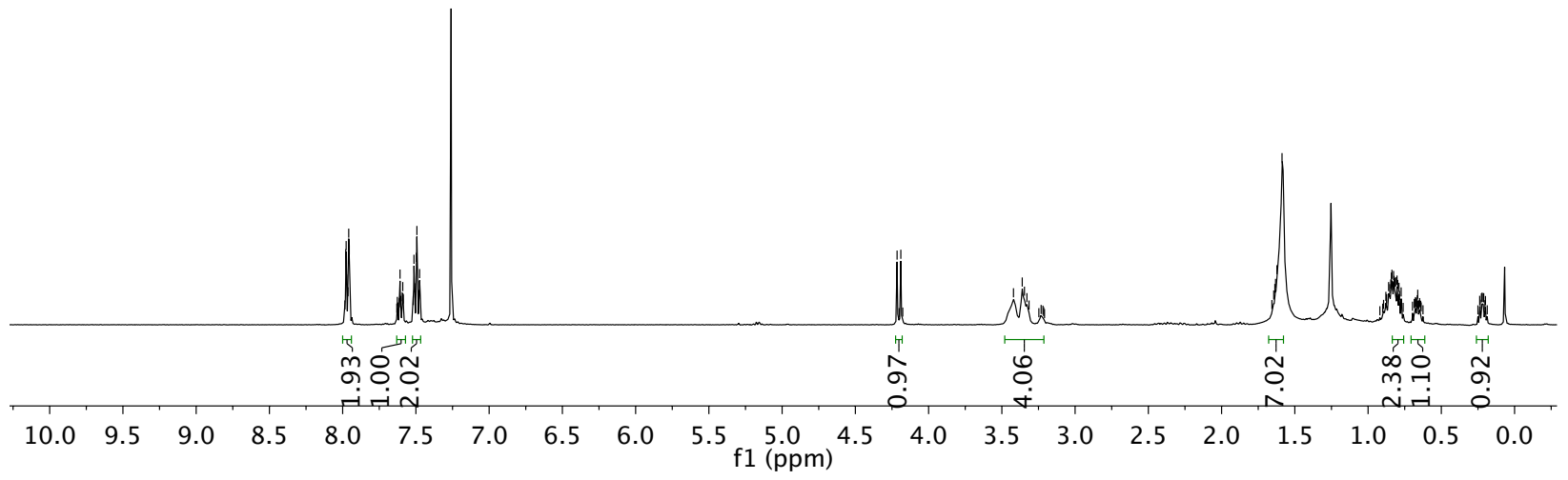<smiles>O=C(c1ccccc1)C(C1CC1)S(=O)(=O)N1CCCCC1</smiles>

54

$\left({ }^{13} \mathrm{C}, 126 \mathrm{MHz}, \mathrm{CDCl}_{3}\right)$

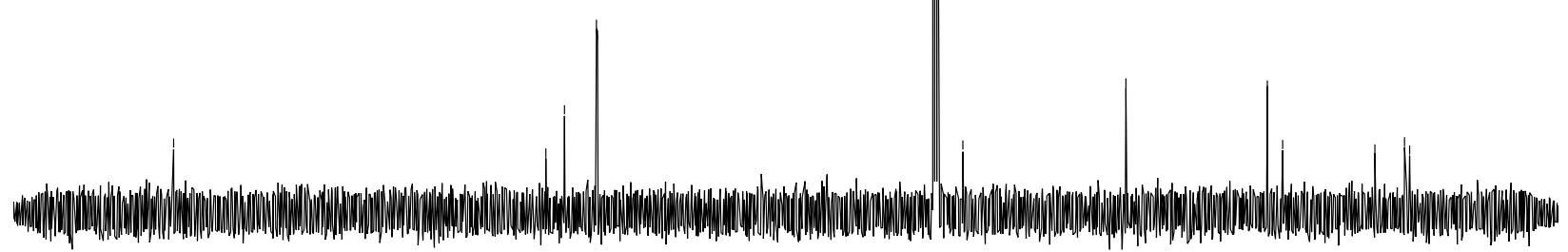

$\begin{array}{llllllllllllllllllllllll}210 & 200 & 190 & 180 & 170 & 160 & 150 & 140 & 130 & 120 & 110 & 100 & 90 & 80 & 70 & 60 & 50 & 40 & 30 & 20 & 10 & 0 & -10\end{array}$ 